Análise e implementação de métodos implícitos e de projeção para escoamentos com superfície livre 


\title{
Análise e implementação de métodos implícitos e de projeção para escoamentos com superfície livre ${ }^{1}$
}

\author{
Cassio Machiaveli Oishi
}

Orientador: Prof. Dr. José Alberto Cuminato

Co-orientador: Prof. Dr. Valdemir Garcia Ferreira

Tese apresentada ao Instituto de Ciências Matemáticas e de Computação - ICMC-USP, como parte dos requisitos para obtenção do título de Doutor em Ciências - Ciências de Computação e Matemática Computacional.

USP - São Carlos

Agosto de 2008

\footnotetext{
${ }^{1}$ Projeto financiado pela FAPESP (n• 03/12612-9)
} 
Dedico às pessoas que mais amo na vida:

minha família. 


\section{Agradecimentos}

Ao professor José Alberto Cuminato pela orientação, apoio, disponibilidade e amizade ao longo destes anos.

Aos professores do LCAD: Valdemir, Murilo, Castelo, Leandro, Nonato e Fabrício.

Ao professor Gustavo Carlos Buscaglia pelas sugestões, paciência e apoio no final deste trabalho.

Aos professores Norberto Mangiavacchi, JY Yuan e Sean McKee pelas discussões sobre meu projeto.

Aos professores Fernando Tavares Pinho e Manuel Alves, da Universidade do Porto, e ao Professor Miguel Nóbrega, da Universidade do Minho, pelo suporte e acolhimento durante meu estágio de doutorado em Portugal.

Aos funcionários do ICMC que sempre foram prestativos ao longo destes anos.

Aos meus amigos, amigas e colegas que acompanharam as alegrias e dificuldades neste período, sempre me apoiando.

A minha família que sempre está presente em minha vida.

A Fapesp pelo financiamento desta pesquisa e a Capes pelo suporte financeiro durante meu estágio no exterior.

Ao ICMC/USP e CEFT/Universidade do Porto pela oportunidade de qualificação e crescimento científico.

Finalmente, e acima de tudo, a Deus pela minha vida. 


\section{Resumo}

No contexto do método MAC e baseado em esquemas de diferenças finitas, este trabalho apresenta três estudos: i) uma análise de estabilidade, ii) o desenvolvimento de técnicas implícitas e, iii) a construção de métodos de projeção para escoamentos com superfície livre.

$\mathrm{Na}$ análise de estabilidade, o principal resultado mostra que o método de Crank-Nicolson torna-se condicionalmente estável quando aplicado para uma malha deslocada com a discretização explícita das condições de contorno do tipo Dirichlet. Entretanto, o mesmo método com condições de contorno implícitas é incondicionalmente estável.

Para obter métodos mais estáveis, formulações implícitas são desenvolvidas para a equação da pressão na superfície livre, derivada da condição de tensão normal. Esta estratégia resulta no acoplamento dos campos de velocidade e pressão, o que exige a introdução de novos métodos de projeção.

Os métodos de projeção assim desenvolvidos resultam em novas metodologias para escoamentos com superfície livre que são apropriados para o tratamento de problemas com baixo número de Reynolds. Além disso, mostra-se que os métodos propostos podem ser aplicados para fluidos viscoelásticos. Novas estratégias são derivadas para obter métodos de projeção de segunda ordem de precisão para escoamentos com superfícies livres.

Além dos resultados teóricos sobre a estabilidade de esquemas numéricos, técnicas implícitas e métodos de projeção, testes computacionais são realizados e comparados para consolidação da teoria apresentada. Os resultados numéricos são obtidos no sistema FREEFLOW.

A eficiência e robustez das técnicas desenvolvidas neste trabalho são demonstradas na solução de problemas tridimensionais complexos com superfície livre e baixo número de Reynolds, incluindo os problemas do jato oscilante e do inchamento do extrudado.

Palavras-chave: Diferenças-finitas, Análise de estabilidade, Técnicas implícitas, Métodos de projeção, Condições de contorno, Escoamentos com superfície livre. 
In the context of the MAC method and based on finite difference schemes, this work presents three studies: i) a stability analysis, ii) the development of implicit techniques, and iii) the construction of projection methods for free surface flows.

In the stability analysis, the main result shows a precise stability restriction on the CrankNicolson method when one uses a staggered grid with Dirichlet explicit boundary conditions. However, the same method with implicit boundary conditions becomes unconditionally stable.

In order to obtain more stable methods, implicit formulations are applied for the pressure equation at the free surface, which is derived from the normal stress condition. This approach results in a coupling of the velocity and pressure fields; hence new projection methods for free surface flows need to be developed.

The developed projection methods result in new methodologies for low Reynolds number free surface flows. It is also shown that the proposed methods can be applied for viscoelastic fluids. New strategies are derived for obtaining second-order accurate projection methods for free surface flows.

In addition to the theoretical results on the stability of numerical schemes, implicit techniques and projection methods, computational tests are carried out and the results compared to consolidate the theory. The numerical results are obtained by the FREEFLOW system.

The efficiency and robustness of the techniques in this work are demonstrated by solving complex tridimensional problems involving free surface and low Reynolds numbers, including the jet buckling and the extrudate swell problems.

Keywords: Finite difference, Stability analysis, Implicit techniques, Projection methods, Boundary conditions, Free surface flows. 
1 Introdução $\quad 1$

2 Conceitos básicos em escoamentos com superfície livre 9

2.1 Introdução . . . . . . . . . . . . . . . . . . . . . . . . . 9

2.2 Discretização temporal . . . . . . . . . . . . . . . . . . . . . . . . . 13

2.3 Malha computacional . . . . . . . . . . . . . . . . . . . . . . . 14

2.4 Partículas marcadoras e células . . . . . . . . . . . . . . . . . 15

2.5 Aproximações da superfície livre . . . . . . . . . . . . . . . . . . . . . . . . . . . . . . . . . . . . . . . . . . .

2.5.1 Caso bidimensional . . . . . . . . . . . . . . . . . . . 17

2.5.2 Caso tridimensional . . . . . . . . . . . . . . . . . . . . . . . 19

2.6 Método de projeção . . . . . . . . . . . . . . . . . . . . . . 21

2.7 Metodologia GENSMAC . . . . . . . . . . . . . . . . . . . 23

2.7.1 Algoritmo do método GENSMAC . . . . . . . . . . . . . . . . 25

2.7.2 Restrição sobre o passo temporal . . . . . . . . . . . . . . . . 25

2.8 Esquemas implícitos na metodologia GENSMAC . . . . . . . . . . . . . 26

3 Estabilidade de métodos numéricos em malha deslocada $\quad 29$

3.1 Introdução . . . . . . . . . . . . . . . . . . . . . . . . 29

3.2 Problema modelo e discretização . . . . . . . . . . . . . . . . . . . . . 32

3.3 Análise de estabilidade . . . . . . . . . . . . . . . . . . . . 36

3.3.1 Método explícito com condições de contorno explícitas . . . . . . . . . . 38

3.3.2 Método de Euler implícito com condições de contorno implícitas . . . . . 39

3.3.3 Método de Euler implícito com condições de contorno explícitas . . . . . 40

3.3.4 Método de Crank-Nicolson com condições de contorno implícitas do tipo 144

3.3.5 Método de Crank-Nicolson com condições de contorno implícitas do tipo 245

3.3.6 Método de Crank-Nicolson com condições de contorno explícitas . . . . . 47

3.4 Verificação . . . . . . . . . . . . . . . . . . . . 52

3.5 Considerações finais . . . . . . . . . . . . . . . . . . . . . . . . 54

4 Métodos de projeção para escoamentos com superfície livre: caso bidimensional

4.1 Introdução . . . . . . . . . . . . . . . . . . . . . . 55

4.2 Um método semi-implícito estável utilizando EI . . . . . . . . . . . . . . . 57

4.2 .1 Algoritmo . . . . . . . . . . . . . . . . . . . . 59 
4.3 Um método semi-implícito estável utilizando $\mathrm{CN} \ldots \ldots$. . . . . . . . . . . 61

4.3 .1 Algoritmo . . . . . . . . . . . . . . . . 62

4.4 Verificação . . . . . . . . . . . . . . . . . . . . . . . 63

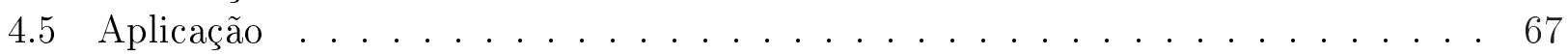

4.6 Considerações finais . . . . . . . . . . . . . . . . . . 70

5 Métodos de projeção para problemas tridimensionais newtonianos e viscoe$\begin{array}{ll}\text { lásticos com superfície livre } & \mathbf{7 1}\end{array}$

5.1 Introdução . . . . . . . . . . . . . . . . . . . . . . 71

5.2 Extensão para problemas tridimensionais newtonianos e viscoelásticos . . . . . 72

5.2 .1 Algoritmo . . . . . . . . . . . . . . . . . . . 79

5.3 Verificação . . . . . . . . . . . . . . . . . . . . 81

5.3 .1 Fluido do tipo Maxwell . . . . . . . . . . . . . . . . . . . . 82

5.3 .2 Fluido do tipo Oldroyd-B . . . . . . . . . . . . . . . . 83

5.4 Aplicações . . . . . . . . . . . . . . . . . . . . . . . . . . 85

5.4 .1 Simulação numérica do jato oscilante . . . . . . . . . . . . . . 85

5.4 .2 Simulação numérica do inchamento do extrudado . . . . . . . . . . . . . 91

5.5 Considerações finais . . . . . . . . . . . . . . . . . . . 100

6 Métodos de projeção de segunda ordem para escoamentos com superfície $\begin{array}{ll}\text { livre } & 101\end{array}$

6.1 Introdução . . . . . . . . . . . . . . . . . . . . . . . . . . . 101

6.2 Método de projeção de ordem dois para a formulação SI-AB/CN . . . . . . . . 106

6.3 Método de projeção de ordem dois para a formulação laplaciano superficial . . 109

6.4 Verificação . . . . . . . . . . . . . . . . . . . . . . . . . . . . . . . . . . . . . . . . . . . . . . . . . . . . .

6.5 Considerações finais . . . . . . . . . . . . . . . . . . . . . 114

7 Considerações Finais $\quad 119$

$\begin{array}{lr}\text { Referências Bibliográficas } & 136\end{array}$

$\begin{array}{ll}\text { A Aspectos importantes da discretização espacial } & 137\end{array}$

A.1 Método GENSMAC . . . . . . . . . . . . . . . . . . . . . . . . 137

A.2 Método semi-implícito estável utilizando EI . . . . . . . . . . . . . . . . 144

A.3 Método semi-implícito estável utilizando CN . . . . . . . . . . . . . . . 145

A.4 Extensão para problemas tridimensionais newtonianos e viscoelásticos . . . . . 147

A.5 Método de projeção de ordem dois para a formulação SI-AB/CN . . . . . . . . . 152

A.6 Método de projeção de ordem dois para a formulação laplaciano superficial . . 152 


\section{Lista de Figuras}

1.1 Resultados experimentais de Nobrega et al. [112] para fluidos viscosos. . . . . . 4

2.1 Ilustração de um escoamento de fluido e seus domínios. . . . . . . . . . . . . . 10

2.2 Exemplos de armazenamento em uma malha deslocada: a) caso bidimensional e b) caso tridimensional. . . . . . . . . . . . . . . . . . . . . . 14

2.3 a) Classificação de células. b) Visualização de um escoamento de xarope de glicose [164]. . . . . . . . . . . . . . . . . . . . . . . . . . . . . . . . . . . . . 16

2.4 Orientação vertical determinada pelos vetores: a) $\mathbf{n}=(1,0)$ ou b) $\mathbf{n}=(-1,0) . \quad$. 17

2.5 Orientação horizontal determinada pelos vetores: a) $\mathbf{n}=(0,1)$ ou b) $\mathbf{n}=(0,-1) .17$

2.6 Orientação inclinada determinada pelos vetores: a) $\mathbf{n}=\left(\frac{\sqrt{2}}{2}, \frac{\sqrt{2}}{2}\right)$, b) $\mathbf{n}=$ $\left(-\frac{\sqrt{2}}{2}, \frac{\sqrt{2}}{2}\right)$, c) $\mathbf{n}=\left(\frac{\sqrt{2}}{2},-\frac{\sqrt{2}}{2}\right)$ e d) $\mathbf{n}=\left(-\frac{\sqrt{2}}{2},-\frac{\sqrt{2}}{2}\right) \ldots \ldots \ldots \ldots$

2.7 Exemplo de uma superfície planar paralela ao plano formado pelos eixos $x y$. Em particular, neste caso $\mathbf{n}=(0,0,1) \ldots \ldots \ldots \ldots$

2.8 Exemplo de uma superfície planar inclinada de $45^{\circ}$. Em particular, neste caso

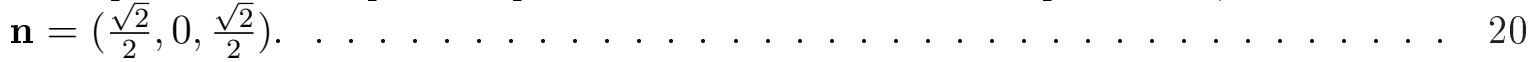

2.9 Exemplo de uma superfície planar inclinada de $60^{\circ}$. Em particular, neste caso

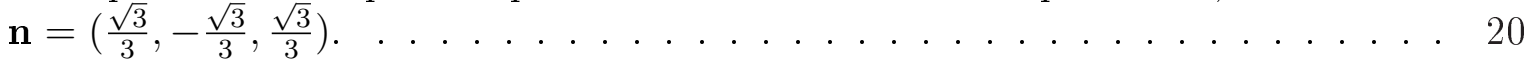

2.10 Ilustração da decomposição de um campo vetorial aplicando o teorema de HelmholtzHodge. . . . . . . . . . . . . . . . . . . . . 22

3.1 Ilustração de uma malha deslocada e classificação de célula em um canal vertical. Neste caso, a velocidade na parede $v_{b}=0$, enquanto que as velocidades internas $v_{1}, v_{2}, \ldots, v_{m-1}, v_{m}$ são calculadas pela equação de quantidade de movimento $(2.22)$

3.2 Malha deslocada para a solução da equação (3.1) com condições de contorno (3.3), em que $u(0, t)=u(1, t)=u_{b}=0 \ldots \ldots \ldots \ldots \ldots \ldots$

3.3 Circulo de Gershgorin $R_{2}$. . . . . . . . . . . . . . . . . . . . . . 42

3.4 Circulo de Gershgorin $R_{1}$ para $\sigma<1 \ldots \ldots \ldots$. . . . . . . . . . . 42

3.5 Circulo de Gershgorin $R_{1}$ para $\sigma>1$. . . . . . . . . . . . . . . . . . . 43

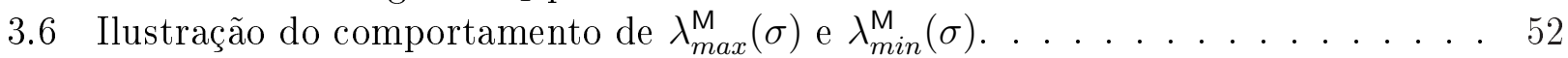

4.1 Comparação entre a solução numérica * e a solução exata $\square$ para o escoamento fountain flow em diferentes tempos utilizando a formulação SI-CN/CCI1. . . . . 
4.2 Perfil da superfície livre e distribuição da velocidade $u$ no tempo $t=4 \mathrm{~s}$ para o escoamento fountain flow. . . . . . . . . . . . . . . . . 67

4.3 Geometria e contornos para o problema do inchamento do extrudado: a) estado transiente e b) estado estacionário. . . . . . . . . . . . . . . . .

4.4 Perfil da superfície livre para o problema do inchamento do extrudado de um fluido newtoniano para a formulação SI-CN/CCI1 em diferentes tempos adimensionais: a) $t=3.0$, b) $t=9.8$ e c) $t=20.0 \ldots \ldots \ldots \ldots$

5.1 a) Vetor normal aponta na direção de uma das direções coordenadas, b) vetor normal aponta na direção formando $45^{\circ}$ com dois eixos coordenados adjacentes, e c) vetor normal aponta na direção formando $60^{\circ} \mathrm{com}$ três eixos coordenados adjacentes. . . . . . . . . . . . . . . . . 76

5.2 Domínio para a simulação do escoamento em um tubo. . . . . . . . . . . . . . 81

5.3 Soluções numéricas obtida nas malhas M1, M2 e M3. As soluções numéricas e exatas foram calculadas em $x=0,-1 \leq y / R \leq 1$ e $z / R=5 \ldots$. . . . . . . .

5.4 Simulação numérica do jato oscilante utilizando as formulações explícita (GENSMAC [155]) e implícita (SI-CN/CCI1, descrita na seção 5.2). Visualização do escoamento em tempos selecionados. ( $R e=0.274$ e $F r=0.478$, fluido newtoni-

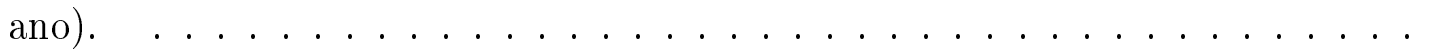

5.5 Simulação numérica do jato oscilante utilizando a formulação implícita (SICN/CCI1, descrita na seção 5.2). Visualização do escoamento em tempos selecionados. $(R e=0.274$ e $F r=0.478$, fluido newtoniano). . . . . . . . . . . . .

5.6 Simulação numérica do jato oscilante utilizando a formulação SI-CN/CCI1. Visualização do escoamento em tempos selecionados. ( $R e=0.5$ e $F r=0.396$, fluido newtoniano). . . . . . . . . . . . . . . . . . .

5.7 Simulação numérica do jato oscilante utilizando a formulação SI-CN/CCI1. Visualização do escoamento em tempos selecionados. $(R e=0.1$ e $F r=0.396$, fluido newtoniano). . . . . . . . . . . . . . . . .

5.8 Simulação numérica do jato oscilante utilizando a formulação SI-CN/CCI1. Visualização do escoamento em tempos selecionados. ( $R e=0.05$ e $F r=0.396$, fluido newtoniano). . . . . . . . . . . . . . . . . .

5.9 Simulação numérica do jato oscilante utilizando a formulação SI-CN/CCI1. Visualização do escoamento em tempos selecionados. $(R e=0.01$ e $F r=0.396$, fluido newtoniano). . . . . . . . . . . . . . . . . . . .

5.10 Simulação numérica do jato oscilante axisimétrico de um fluido newtoniano e do tipo Maxwell utilizando a formulação implícita (SI-EI/CCE, descrita na seção $5.2)$. Visualização do escoamento em tempos selecionados. ( $R e=0.5$ e $W e=$ $0.5)$.

5.11 Simulação numérica do jato oscilante quadrado de um fluido newtoniano e do tipo Maxwell utilizando a formulação implícita (SI-EI/CCE, descrita na seção 5.2). Visualização do escoamento em tempos selecionados. $(R e=0.5$ e $W e=0.5)$. 
5.12 Simulação numérica do jato oscilante axisimétrico de um fluido newtoniano e do tipo Maxwell utilizando a formulação implícita (SI-EI/CCE, descrita na seção 5.2). Visualização do escoamento em tempos selecionados. ( $R e=1.3$ e $W e=$ $0.5)$

5.13 Simulação numérica do jato oscilante quadrado de um fluido newtoniano e do tipo Maxwell utilizando a formulação implícita (SI-EI/CCE, descrita na seção 5.2). Visualização do escoamento em tempos selecionados. $(R e=1.3$ e $W e=0.5)$.

5.14 Simulação numérica do fenômeno do inchamento do extrudado não-estacionário para vários valores de $W e_{\text {efetivo }}$ : 0.2 (primeira coluna); 0.5 (segunda coluna); 0.8 (terceira coluna). Visualização do escoamento em tempos selecionados $(R e=0.1$,

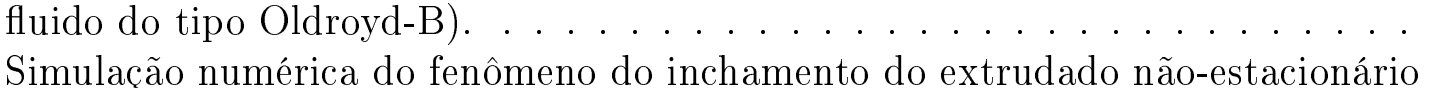
para vários valores de $W e_{\text {efetivo }}$ : 0.2 (primeira coluna); 0.5 (segunda coluna); 0.8 (terceira coluna). Visualização do escoamento em tempos selecionados $(R e=$ 0.05, fluido do tipo Oldroyd-B). . . . . . . . . . . . . . . . .

5.16 Simulação numérica do fenômeno do inchamento do extrudado não-estacionário para vários valores de $W e$ : 0.1 (primeira coluna); 0.2 (segunda coluna); 0.4 (terceira coluna). Visualização do escoamento em tempos selecionados $(R e=1.0$, fluido do tipo Maxwell). . . . . . . . . . . . . . . . . . . . .

6.1 Ilustração dos vetores normal $(\mathbf{n}(s))$ e tangencial $(\mathbf{m}(s))$ em uma curva $\omega$ parametrizada pelo comprimento de arco.

A.1 Localização das componentes de velocidade e da quantidade escalar em células próximas à superfície livre. Orientação vertical determinada pelos vetores: a) $\mathbf{n}=(1,0)$ ou b) $\mathbf{n}=(-1,0) \ldots \ldots \ldots \ldots \ldots \ldots \ldots$

A.2 Localização das componentes de velocidade e da quantidade escalar em células próximas à superfície livre. Orientação horizontal determinada pelos vetores: a) $\mathbf{n}=(0,1)$ ou b) $\mathbf{n}=(0,-1) \ldots \ldots \ldots \ldots \ldots \ldots \ldots \ldots$

A.3 Localização das componentes de velocidade e da quantidade escalar em células próximas à superfície livre. Orientação inclinada determinada pelos vetores: a) $\left.\left.\mathbf{n}=\left(\frac{\sqrt{2}}{2}, \frac{\sqrt{2}}{2}\right), \mathrm{b}\right) \mathbf{n}=\left(-\frac{\sqrt{2}}{2}, \frac{\sqrt{2}}{2}\right), \mathrm{c}\right) \mathbf{n}=\left(\frac{\sqrt{2}}{2},-\frac{\sqrt{2}}{2}\right)$ e d) $\mathbf{n}=\left(-\frac{\sqrt{2}}{2},-\frac{\sqrt{2}}{2}\right) . \quad \ldots . .140$

A.4 Célula $[\mathbf{S}]$ com apenas a face superior em contato com uma célula $[\mathbf{E}]$. . . . . . 149 


\section{Lista de Tabelas}

2.1 Esquemas de discretização temporal. Ex: método explícito, EI: método de Euler implícito, CN: método de Crank-Nicolson e AB/CN: método de AdamsBashforth/Crank-Nicolson. . . . . . . . . . . . . . . . . . . . 13

3.1 Escoamento confinado $(R e=0.1) \ldots \ldots \ldots \ldots \ldots$

3.2 Escoamento confinado $(R e=0.01) \ldots \ldots \ldots \ldots \ldots$

4.1 Escoamento com superfície livre $(R e=0.1) \ldots \ldots \ldots \ldots$. . . . . . . . 65

4.2 Escoamento com superfície livre $(R e=0.01) \ldots \ldots \ldots$. . . . . . . . . . 65

4.3 Escoamento com superfície livre $(R e=0.1)$, utilizando as malhas M1, M2 e M3.

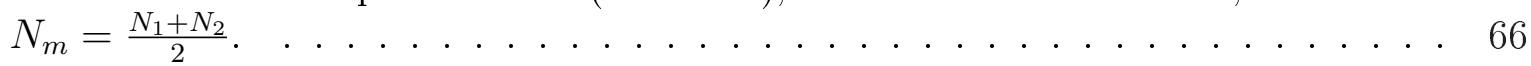

4.4 Resultados do raio do inchamento do extrudado $S_{r}$ para $R e=0.01$ utilizando as formulações SI-EI/CCE e SI-CN/CCI1 em diferentes malhas (M2, M3 e M4). . .

5.1 Erros obtidos nas malhas M1, M2 e M3 para a formulação SI-EI/CCE no escoamento em um tubo para o fluido do tipo Maxwell. . . . . . . . . . . . . . . 83

5.2 Comparação da performance dos esquemas explícito e implícito na malha M0. . 84

5.3 Erros nas malhas M1, M2 e M3 para o escoamento em um tubo com o método SI-CN/CCI1. . . . . . . . . . . . . . . . . . . . 85

5.4 Resultados do escoamento do inchamento do extrudado para um fluido do tipo Maxwell. Os valores numéricos de $S_{r}$ foram obtidos pela formulação SI-EI/CCE, enquanto que os teóricos pela lei de Tanner [149]. . . . . . . . . . . . . . . 96

6.1 Erros para a formulação SI-AB/CN da seção 6.2. $\left(R e=0.1, \delta t_{1}=2.0 \times 10^{-3} s\right.$, $\delta t_{2}=1.0 \times 10^{-3} s$ e $\left.\delta t_{3}=5.0 \times 10^{-4} s\right) \ldots \ldots \ldots \ldots \ldots \ldots \ldots$

6.2 Erros para a formulação SI-AB/CN da seção 6.2 sem a extrapolação linear no tempo (6.9) para as condições de contorno de $\widetilde{\mathbf{u}}$ na solução da equação (6.2). $\left(R e=0.1, \delta t_{1}=2.0 \times 10^{-3} s, \delta t_{2}=1.0 \times 10^{-3} s\right.$ e $\left.\delta t_{3}=5.0 \times 10^{-4} s\right) \ldots \ldots . . .115$

6.3 Erros para a formulação SI-AB/CN da seção $6.2 \mathrm{com}$ a equação da pressão na superfície livre discretizada no nível de tempo $(n+1) . \quad\left(R e=0.1, \delta t_{1}=\right.$ $2.0 \times 10^{-3} s, \delta t_{2}=1.0 \times 10^{-3} s$ e $\left.\delta t_{3}=5.0 \times 10^{-4} s\right) \ldots \ldots \ldots \ldots 116$

6.4 Erros para a formulação SI-AB/CN da seção 6.2 sem a extrapolação linear no tempo (6.9) e com a equação da pressão na superfície livre discretizada no nível de tempo $(n+1) .\left(R e=0.1, \delta t_{1}=2.0 \times 10^{-3} s, \delta t_{2}=1.0 \times 10^{-3} s\right.$ e $\left.\delta t_{3}=5.0 \times 10^{-4} s\right) .116$ 
6.5 Erros para a formulação laplaciano superficial da seção 6.3. $\left(R e=0.1, \delta t_{1}=\right.$ $2.0 \times 10^{-3} s, \delta t_{2}=1.0 \times 10^{-3} s$ e $\left.\delta t_{3}=5.0 \times 10^{-4} s\right) \ldots \ldots \ldots \ldots . \ldots \ldots 17$

6.6 Erros para a formulação laplaciano superficial da seção 6.3 sem a extrapolação linear no tempo (6.9) para as condições de contorno de $\widetilde{\mathbf{u}}$ na solução da equação (6.2). $\left(R e=0.1, \delta t_{1}=2.0 \times 10^{-3} s, \delta t_{2}=1.0 \times 10^{-3} s\right.$ e $\left.\delta t_{3}=5.0 \times 10^{-4} s\right) . \quad . \quad .117$

6.7 Erros para a laplaciano superficial da seção 6.3 com a equação da pressão na superfície livre discretizada no nível de tempo $(n+1)$. $\left(R e=0.1, \delta t_{1}=2.0 \times\right.$ $10^{-3} s, \delta t_{2}=1.0 \times 10^{-3} s$ e $\left.\delta t_{3}=5.0 \times 10^{-4} s\right) \ldots \ldots \ldots \ldots \ldots$

6.8 Erros para a formulação laplaciano superficial da seção 6.3 sem a extrapolação linear no tempo (6.9) e com a equação da pressão na superfície livre discretizada no nível de tempo $(n+1) . \quad\left(R e=0.1, \delta t_{1}=2.0 \times 10^{-3} s, \delta t_{2}=1.0 \times 10^{-3} s\right.$ e

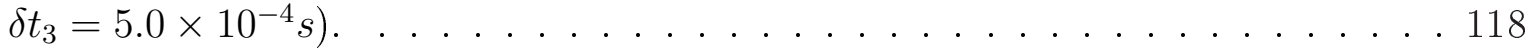

A.1 Escoamento confinado $(R e=0.1) \ldots \ldots \ldots \ldots$. . . . . . . . . . . . . . . . . . . . . .

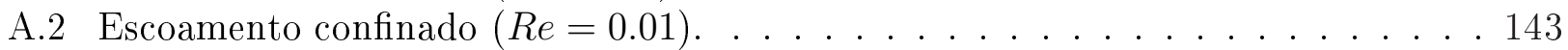

A.3 Valores de $\psi_{1}, \psi_{2}, h\left(\widetilde{u}_{m}\right)$ e $h\left(u_{m}^{(n)}\right)$ na equação de diferenças finitas (A.28) para os diferentes valores do vetor $\mathbf{n} . \ldots \ldots$. . . . . . . . . . . . . . 152 


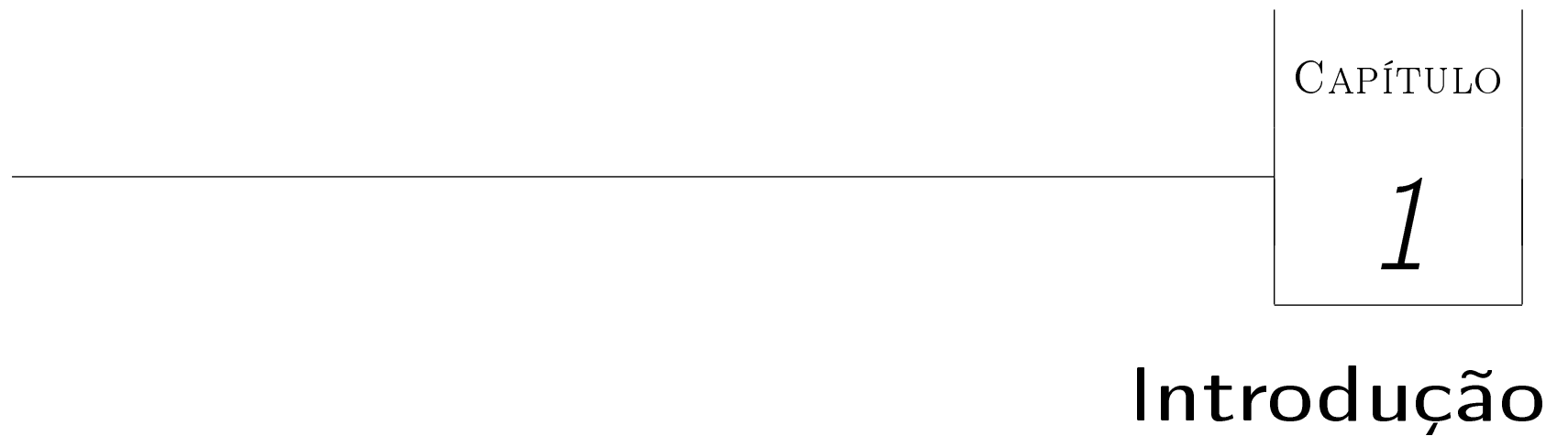

A Dinâmica dos Fluidos Computacional (DFC) é uma área de pesquisa consolidada e importante, com numerosas aplicações na indústria e ciência. Em particular, fenômenos da DFC para problemas de escoamentos incompressíveis aparecem em várias áreas de pesquisa.

Nas últimas décadas, pesquisadores de diversas áreas de aplicação têm se dedicado ao desenvolvimento de métodos numéricos em DFC. Exemplos dessas áreas aparecem nas indústrias automotiva, eletrônica, nuclear, biomédica, farmacêutica, alimentícia, aeroespacial, química, entre outras.

Simulações computacionais apresentam vários benefícios para a indústria. Um benefício importante é a economia de tempo para explorar fenômenos que ocorrem nos escoamentos de fluidos. Geralmente, experimentos em dinâmica dos fluidos consomem muito tempo, e na maioria da vezes, são extremamente caros; com a DFC muitas possibilidades podem ser exploradas. A simulação em DFC fornece informações úteis de diversas propriedades físicas do escoamento, como para os campos de velocidade, pressão, temperatura, concentração de substâncias e muitas outras, enquanto que testes experimentais fornecem dados apenas em posições onde os instrumentos de medida são instalados. Entretanto, o uso de simulações numéricas em DFC não elimina a necessidade de testes experimentais, mas é uma ferramenta poderosa na compreensão dos escoamentos. Em muitos casos, resultados experimentais e numéricos são comparados e analisados para a obtenção de mais informações sobre o problema físico em questão.

Uma classe de escoamentos muito investigada pela DFC são os escoamentos externos, onde o fluido não está totalmente confinado por paredes, podendo apresentar superfícies livres, isto é, 
o fluido está em contato direto com a atmosfera. Escoamentos de fluidos com superfícies livres apresentam dificuldades adicionais na simulação numéricas, pois além de resolver as equações governantes, a superfície livre precisa ser determinada ao mesmo tempo.

O desenvolvimento de métodos numéricos para a simulação de escoamentos com superfícies livres tem sido uma área de pesquisa atrativa ultimamente. Isto é justificado, pois escoamentos com superfícies livres são fundamentais em vários problemas da engenharia e ciências afins, como por exemplo na agitação da água em recipientes vibrantes, em escoamentos de marés em baías, em escoamentos com superfície livre em torno de um cilindro, entre outros problemas. Obviamente, a natureza desses escoamentos com superfícies livres varia significantemente de acordo com o problema físico que eles descrevem. Portanto, não é surpresa que uma imensa variedade de métodos computacionais tenha sido desenvolvida para a simulação numérica desses escoamentos.

Métodos computacionais que utilizam uma malha estacionária (fixa) e uma estratégia para identificar a localização da superfície livre no domínio são frequentemente utilizados por razões de eficiência computacional e flexibilidade na extensão de códigos. Um exemplo destes métodos é a combinação das técnicas de avanço de fronteira (front trancking) com a MAC (Marker-AndCell). A primeira foi bem discutida por Glimm e seus co-autores [21, 56, 57] e por Tryggvason e seus co-autores $[41,167,168]$, e a segunda foi primeiramente introduzida por Harlow e Welch $[4]$.

A técnica de avanço de fronteira envolve elementos que se movem sobre uma malha euleriana (fixa). Neste caso a superfície livre é considerada como um conjunto de pontos ligados entre si formando uma malha superficial não-estruturada. Desta forma, esta malha não-estruturada move-se sobre uma malha fixa que armazena as variáveis de interesse do escoamento. Outros esquemas que combinam técnicas computacionais para lidar com problemas com superfície livre podem ser encontrados em [55] para a técnica de captura de fronteira (front capturing), [128, 82] para o método de Volume-de-Fluido (VOF-Volume-Of-Fluid), [172, 121, 16, 25] para esquemas de conjunto de nível (level set), [43, 95] para o método de fluido fantasma (Ghost fluid), [90] para metodologia de fronteiras imersas (immersed boundary), [179, 178] para técnica de interface nítida (sharp interface), entre muitos outros.

Em 1965, Harlow e Welch [66] apresentaram uma formulação adequada para o tratamento de escoamentos com superfície livre, o famoso método MAC. Este método foi uma variação do esquema PIC (Particle-In-Cell) desenvolvido em 1957 por Evans e Harlow [42]. O método MAC é uma técnica baseada em diferenças finitas nas variáveis primitivas (pressão e velocidade) para a simulação de escoamentos de fluidos incompressíveis. Uma característica importante deste 
método é o uso de partículas virtuais lagrangeanas, que movimentam-se de uma célula para outra, de acordo com o último campo de velocidade calculado.

O método MAC foi inicialmente usado para problemas bidimensionais, pois este esquema exigia um consumo de memória e tempo de processamento considerável para acomodar o número necessário de partículas marcadoras. Em 1970, Amsden e Harlow [4] desenvolveram um método MAC simplificado, o SMAC (Simplified Marker-And-Cell), no qual algumas dificuldades do método de Harlow e Welch [66] foram eliminadas. Muitas variações do método SMAC apareceram ao longo dos anos, e ainda aparecem na literatura específica. Primeiramente, o cálculo da pressão na superfície livre foi modificado, como descreve os trabalhos de Viecelli [171], Chan e Street [17] e Hirt e Nichols [71]. Métodos com melhor precisão na captura da superfície livre também foram desenvolvidos.

Variações do método MAC, onde essas modificações são implementadas, foram apresentados na década de 70, nos métodos SUMMAC (Stanford University Modified Marker-And-Cell) [17] e ALE (Arbitrary-Lagrangian-Eulerian) [70]; na década de 80 no esquema TUMMAC (Tokyo University Modified Marker-And-Cell) [103, 102]; e na década de 90 no método GENSMAC (GENeralized Simplified Marker-And-Cell) [163]. Mais recentemente, outros autores têm usado essencialmente as mesmas idéias do método MAC: em técnicas lagrangeana-eulerianas na simulação de escoamentos em domínios arbitrários [170], para simular o problema do impacto de uma gota [105], em métodos de segunda ordem para a reconstrução de interfaces [81], entre outras. Recentemente, McKee et al. [100] descreveram a história, interpretação, variações e aplicações do método MAC. Portanto, métodos do tipo MAC são ainda freqüentemente estudados na literatura, pois aplicações envolvendo escoamentos com superfície livre são comuns no dia-a-dia.

O método GENSMAC de Tomé e McKee [163] foi implementado com o objetivo de resolver problemas de escoamentos transientes incompressíveis com superfícies livres. No contexto de diferenças finitas, este esquema utiliza variáveis primitivas, e sua potencialidade foi reconhecida na DFC em [60, 33, 77] entre outros.

Variações do método GENSMAC foram apresentadas por: Sousa et al. [145] que utilizaram o método MAC para escoamento tridimensional multifásico com superfícies livres, Ferreira et al. [47] que realizaram simulações de problemas turbulentos com superfícies livres, Mangiavacchi et al. [98] que implementaram novas técnicas em escoamentos axisimétrico e bidimensionais com superfícies livres quando a tensão superficial é significante, Ferreira et al. [48] que adaptaram o método MAC para calcular escoamentos confinados e com superfícies livres a altos e baixos números de Reynolds, Tomé et al. [160] que aplicaram as técnicas do método MAC na simu- 
lação de escoamentos axisimétrico com superfícies livres utilizando fluido do tipo Oldroyd-B. Recentemente, Tomé e seus co-autores [158, 157] e Paulo et al. [123] apresentaram resultados numéricos para problemas viscoelásticos com superfícies livres, entre outras novas aplicações do método MAC. Além disso, Ferreira e seus co-autores [45, 44, 46] aplicaram estratégias upwind na metodologia GENSMAC.

O método GENSMAC e suas variações citadas acima utilizam uma formulação explícita, e conseqüentemente o tamanho do passo temporal na simulação numérica é sujeito à restrição parabólica de estabilidade, como pode ser visto no trabalho de Tomé e Mckee [163]. Esta restrição indica que o passo temporal permitido é calculado de acordo com o parâmetro adimensional número de Reynolds (razão entre as forças inerciais e viscosas) e o espaçamento da malha. Em muitos escoamentos de fluidos as forças viscosas são predominantes, e o número de Reynolds é freqüentemente menor que 1. Exemplos onde o número de Reynolds é muito baixo aparecem constantemente em problemas nas indústrias química e de polímeros, tais como: escoamentos de plásticos, de vidros, de polímeros, preechimento de moldes ou modelos, inchamento do extrudado, escoamentos de jatos e etc. Exemplos de escoamentos viscosos podem ser vistos na figura 1.1.
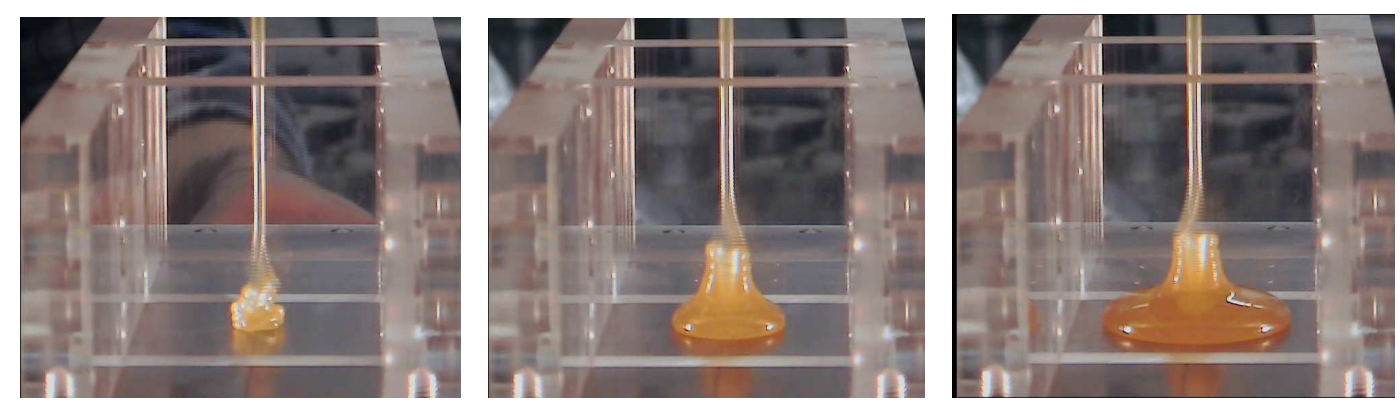

Figura 1.1: Resultados experimentais de Nobrega et al. [112] para fluidos viscosos.

Neste tipo de simulação, técnicas explícitas, tais como o método GENSMAC, requerem muitos passos no tempo e conseqüentemente consomem um alto tempo de processamento. Desta forma, métodos numéricos para tratar escoamentos de fluidos viscosos são ferramentas importantes na obtenção de resultados em DFC.

Nas últimas décadas, autores têm apresentado métodos baseados no esquema MAC com formulações implícitas. A maioria desses métodos resolvem as equações de Navier-Stokes em variáveis primitivas utilizando formulações acopladas ou segregadas. Os métodos que resolvem simultaneamente estas equações a cada passo no tempo são conhecidos como acoplados, e os métodos que resolvem as equações separadamente, como segregados. 
Métodos acoplados são altamente influenciados pelas não-linearidades dos termos advectivos, além de apresentarem dificuldades de implementação e alto custo computacional. Um método do tipo acoplado foi apresentado por Pracht [129], denominado MACRL (Marker-AndCell-Reynolds-Low), o qual é uma versão implícita do esquema MAC. Entretanto, em cada passo temporal um conjunto completo de equações não-lineares para as variáveis primitivas tem que ser resolvido, e isto pode consumir muito tempo de processamento. Portanto, a estratégia do desacoplamento é muito eficiente e tem sido constantemente utilizada por muitos autores. Um método baseado nesta estratégia para escoamentos a baixo número de Reynolds $(R e<<1)$ foi proposto por Golafashani [58].

Um exemplo de método desacoplado baseado no esquema MAC é o SIMAC (Semi-Implicit Marker-And-Cell) proposto por Armenio [5]. O método SIMAC é uma modificação do método MAC para a solução de escoamentos de fluidos com superfície livre a alto número de Reynolds usando uma discretização numérica semi-implícita. A formulação semi-implícita contribui na eficiência dos esquemas que resolvem as equações de Navier-Stokes de forma desacoplada. Este fato é justificado, pois em métodos semi-implícitos, os termos advectivos são tratados explicitamente, enquanto que os termos viscosos implicitamente. Entretanto, como o objetivo do método SIMAC é simular escoamentos a alto número de Reynolds, este esquema simplifica a condição de contorno da tensão normal, admitindo que a pressão na superfície livre é igual a zero. Os trabalhos de Nichols et al. [110] e Lemos [88] também apresentam simplificações nas condições de contorno na superfície livre. Como o objetivo do presente trabalho é a simulação de escoamentos de fluidos a baixo número de Reynolds, a simplificação utilizada por Armenio e outros autores, não pode ser aplicada.

Outros métodos que utilizam técnicas implícitas para solução de Navier-Stokes foram descritos em [14, 37, 169, 9, 99, 173, 86, 12, 7, 65] entre muitos outros. Portanto, a implementação de estratégias implícitas tem sido muito pesquisada atualmente, motivando o estudo e implementação desse tipo de esquema.

Fundamentado na metodologia MAC este texto é dividido em três principais partes, que derivaram resultados originais a esta tese:

1. No início desta pesquisa, apresenta-se um estudo detalhado sobre a estabilidade de métodos numéricos em malhas do tipo MAC. Será verificado, através de um problema modelo e ferramentas de álgebra linear aplicada, como o tratamento temporal das condições de contorno em fronteiras rígidas pode afetar a estabilidade de esquemas numéricos bem co- 
nhecidos na literatura por sua estabilidade. Os resultados teóricos e numéricos referentes a esta pesquisa são apresentados no capítulo 3 .

2. Logo após a primeira etapa, inicia-se a análise de métodos de projeção e de técnicas implícitas em escoamentos bidimensionais com superfícies livres. Duas estratégias são apresentadas, derivando métodos estáveis e eficientes em escoamentos a baixo número de Reynolds. Os resultados estão reunidos no capítulo 4. No capítulo 5, as idéias para o caso bidimensional são estendidas para escoamentos tridimensionais newtonianos e viscoelásticos. Os capítulos referentes a esta etapa apresentam resultados numéricos de problemas viscosos com superfícies livres.

3. Na última etapa desta tese, finaliza-se os estudos de métodos de projeção em escoamentos com superfície livre derivando-se estratégias para construir métodos de segunda ordem no tempo. Estes métodos e mais resultados numéricos são descritos no capítulo 6.

Os capítulos 3, 4, 5 e 6, apesar de conterem temas interrelacionados, são apresentados separadamente incluindo introdução, resultados numéricos e considerações finais em cada capítulo. No capítulo 2 apresentam-se conceitos básicos em escoamentos com superfície livre. O último capítulo desta tese é dedicado às considerações finais, enquanto que no Apêndice A, as equações de diferenças finitas utilizadas neste trabalho são detalhadas.

Alguns dos resultados desta tese já foram publicados na literatura. Por exemplo, os resultados do capítulo 3 foram reunidos no artigo

- Oishi et al., Stability of numerical schemes on staggered grids, Numerical Linear Algebra with Applications, aceito para publicação, 2008, DOI: 10.1002/nla.597,

do capítulo 4 no artigo

- Oishi et al., A stable semi-implicit method for free surface flows, Transaction of the ASME - Journal of Applied Mechanics, 73: 940-947, 2006,

e do capítulo 5 no artigo

- Oishi et al., An implicit technique for solving 3D low Reynolds number moving free surface flows, Journal of Computational Physics, 227: 7446-7468, 2008. 
Atualmente, o grupo de DFC do Laboratório de Computação de Alto Desempenho (LCAD) instalado no Instituto de Ciências Matemáticas e de Computação tem se dedicado ao desenvolvimento de um sistema de simulação para escoamentos transientes incompressíveis com superfície livre. Esse sistema foi denominado FREEFLOW, o qual consiste de um sistema computacional para modelagem, simulação e visualização de escoamentos de fluidos com superfície livre. Os módulos que compõem este sistema foram implementados utilizando a linguagem $\mathrm{C}$ sobre a plataforma LINUX. A versão original do FREEFLOW foi publicada na literatura no ano 2000, no trabalho de Castelo et al. [15]. Após esse trabalho, diversas modificações do sistema FREEFLOW foram realizadas. Portanto, como esta pesquisa está incluída no LCAD, todas as simulações numéricas apresentadas nesta tese foram resultados do sistema FREEFLOW. As novas técnicas descritas neste trabalho foram implementadas na parte principal deste sistema, denominada "simulador". 


\section{Conceitos básicos em escoamentos com superfície livre}

Este capítulo tem por objetivo introduzir os principais conceitos relativos ao método MAC para a solução numérica das equações de Navier-Stokes com superfície livre. A leitura deste capítulo deverá instrumentar o leitor com todos os requisitos necessários para o entendimento do restante do trabalho.

A formulação MAC exibe muitas particularidades, somente as mais relevantes serão apresentadas neste capítulo.

\subsection{Introdução}

Em forma adimensional conservativa, as equações de Navier-Stokes para escoamentos viscosos, incompressíveis e newtonianos podem ser escritas como

$$
\begin{gathered}
\frac{\partial \mathbf{u}}{\partial t}+\nabla \cdot(\mathbf{u u})=-\nabla p+R e^{-1} \nabla^{2} \mathbf{u}+\left(F r^{2}\right)^{-1} \mathbf{g} \quad \text { em } \quad[0, T] \times \Omega, \\
\nabla \cdot \mathbf{u}=0 \quad \text { em } \quad[0, T] \times \Omega,
\end{gathered}
$$

onde $\mathbf{u}=\mathbf{u}(\mathbf{x}, t)$ é o campo de velocidade, $p=p(\mathbf{x}, t)$ é a pressão, $\mathbf{g}$ é o campo gravitacional, $R e=L U / \nu$ é o número de Reynolds e $F r=U / \sqrt{g L}$ é o número de Froude. $L$ e $U$ são, 
respectivamente, as escalas de comprimento e velocidade, e $\nu$ é o coeficiente de viscosidade cinemática do fluido. $\Omega$ é um domínio em $\mathbb{R}^{d}(d=2$ ou 3 em problemas práticos) e $[0, T]$ é um intervalo de tempo dado. Detalhes sobre a adimensionalização destas equações podem ser encontrados em [113].

Para resolver as equações (2.1) e (2.2), condições iniciais e de contorno apropriadas precisam ser impostas. Em $t=t_{0}$, as condições iniciais são definidas como

$$
\mathbf{u}=\mathbf{u}_{0} \quad \text { e } \quad p=p_{0}
$$

com $\mathbf{u}_{0}$ e $p_{0}$ satisfazendo as equações $(2.1)$ e (2.2) e também as condições de contorno, que podem ser dos seguintes tipos (veja figura 2.1):

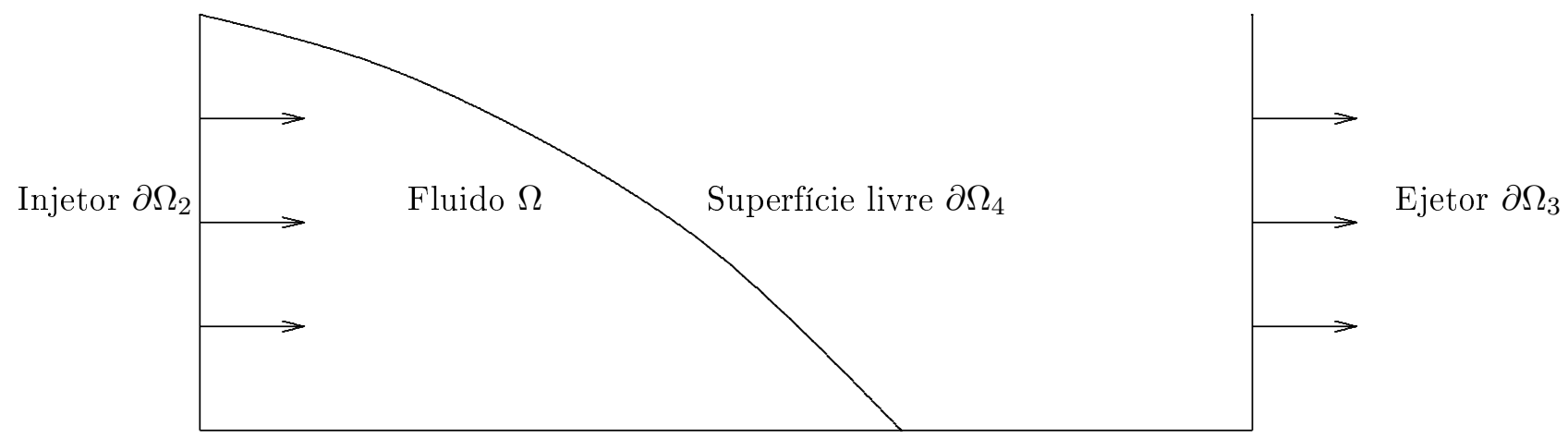

Contorno rígido $\partial \Omega_{1}$

Figura 2.1: Ilustração de um escoamento de fluido e seus domínios.

- Condições de contorno sem deslizamento, isto é,

$$
\mathbf{u}=0 \quad \text { em } \quad \partial \Omega_{1}
$$

onde $\partial \Omega_{1}$ é o contorno rígido (contorno de parede);

- Condições de contorno sem aderência, definidas como

$$
\mathbf{u} \cdot \mathbf{n}=0 \quad \text { em } \quad \partial \Omega_{1}
$$

e condições de contorno do tipo Neumann homogênea para a velocidade tangencial. Neste trabalho, convenciona-se que $\mathbf{n}$ é o vetor normal unitário, externo ao contorno, e m é o vetor tangente ao contorno; 
- Condições de contorno preescritas no injetor $\partial \Omega_{2}$ (entrada de fluido), ou seja,

$$
\mathbf{u} \cdot \mathbf{n}=U \quad \text { e } \quad \mathbf{u} \cdot \mathbf{m}=0 \quad \text { em } \quad \partial \Omega_{2}
$$

- Condições de contorno do tipo Neumann homogênea no ejetor $\partial \Omega_{3}$ (saída de fluido);

- Condições para contornos em movimento: na superfície livre é necessário impor condições nos campos de velocidade e pressão. Essas condições, na ausência de tensão superficial são escritas como (veja detalhes em [10])

$$
\begin{gathered}
\mathbf{n}^{T} \cdot \mathrm{T} \cdot \mathbf{n}=0 \quad \text { em } \quad \partial \Omega_{4}, \\
\mathbf{m}^{T} \cdot \mathrm{T} \cdot \mathbf{n}=0 \quad \text { em } \quad \partial \Omega_{4},
\end{gathered}
$$

onde $\partial \Omega_{4}$ é a superfície livre. O tensor total T é definido como

$$
\mathrm{T}=-p \mathbf{l}+\boldsymbol{\tau}
$$

em que I é o tensor identidade e $\tau$ é o tensor de tensão cisalhante. Para fluidos newtonianos, este tensor é definido como

$$
\boldsymbol{\tau}=\frac{1}{R e}\left[\nabla \mathbf{u}+(\nabla \mathbf{u})^{T}\right]
$$

Em problemas bidimensionais, isto é, nos casos em que $\partial \Omega_{4} \subset \mathbb{R}^{2}$, o vetor normal à superfície livre é denotado por $\mathbf{n}=\left(n_{x}, n_{y}\right)$ e o tangencial $\mathbf{m}=\left(m_{x}, m_{y}\right)$. Substituindo o tensor total dado por (2.9) com o tensor de tensão cisalhante (2.10), nas equações (2.7) e (2.8), obtém-se, respectivamente as seguintes equações

$$
\begin{aligned}
& -p+\frac{2}{R e}\left[\frac{\partial u}{\partial x} n_{x}^{2}+\frac{\partial v}{\partial y} n_{y}^{2}+\left(\frac{\partial u}{\partial y}+\frac{\partial v}{\partial x}\right) n_{x} n_{y}\right]=0 \quad \text { em } \quad \partial \Omega_{4}, \\
& 2 \frac{\partial u}{\partial x} n_{x} m_{x}+2 \frac{\partial v}{\partial y} n_{y} m_{y}+\left[\frac{\partial u}{\partial y}+\frac{\partial v}{\partial x}\right]\left(n_{y} m_{x}+n_{x} m_{y}\right)=0 \quad \text { em } \quad \partial \Omega_{4} .
\end{aligned}
$$

Para problemas tridimensionais, é necessário adicionar a equação

$$
\mathbf{m}_{1}^{T} \cdot \mathbf{T} \cdot \mathbf{n}=0 \quad \text { em } \quad \partial \Omega_{4},
$$


ao sistema de equações (2.7)-(2.8), reescrevendo o vetor normal como $\mathbf{n}=\left(n_{x}, n_{y}, n_{z}\right)$, e os vetores tangenciais como $\mathbf{m}=\left(m_{x}, m_{y}, m_{z}\right)$ e $\mathbf{m}_{1}=\left(m_{1 x}, m_{1 y}, m_{1 z}\right)$. Neste caso, em coordenadas cartesianas, as equações (2.7), (2.8) e (2.13) tornam-se respectivamente

$$
\begin{aligned}
& -p+\frac{2}{R e}\left[\frac{\partial u}{\partial x} n_{x}^{2}+\frac{\partial v}{\partial y} n_{y}^{2}+\frac{\partial w}{\partial z} n_{z}^{2}+\left(\frac{\partial u}{\partial y}+\frac{\partial v}{\partial x}\right) n_{x} n_{y}+\left(\frac{\partial u}{\partial z}+\frac{\partial w}{\partial x}\right) n_{x} n_{z}\right. \\
& \left.+\left(\frac{\partial v}{\partial z}+\frac{\partial w}{\partial y}\right) n_{y} n_{z}\right]=0 \quad \text { em } \quad \partial \Omega_{4} \\
& 2 \frac{\partial u}{\partial x} n_{x} m_{x}+2 \frac{\partial v}{\partial y} n_{y} m_{y}+2 \frac{\partial w}{\partial z} n_{z} m_{z}+\left[\frac{\partial u}{\partial y}+\frac{\partial v}{\partial x}\right]\left(n_{x} m_{y}+n_{y} m_{x}\right) \\
& +\left[\frac{\partial u}{\partial z}+\frac{\partial w}{\partial x}\right]\left(n_{x} m_{z}+n_{z} m_{x}\right)+\left[\frac{\partial v}{\partial z}+\frac{\partial w}{\partial y}\right]\left(n_{y} m_{z}+n_{z} m_{y}\right)=0 \quad \text { em } \quad \partial \Omega_{4}, \\
& 2 \frac{\partial u}{\partial x} n_{x} m_{1 x}+2 \frac{\partial v}{\partial y} n_{y} m_{1 y}+2 \frac{\partial w}{\partial z} n_{z} m_{1 z}+\left[\frac{\partial u}{\partial y}+\frac{\partial v}{\partial x}\right]\left(n_{x} m_{1 y}+n_{y} m_{1 x}\right) \\
& +\left[\frac{\partial u}{\partial z}+\frac{\partial w}{\partial x}\right]\left(n_{x} m_{1 z}+n_{z} m_{1 x}\right)+\left[\frac{\partial v}{\partial z}+\frac{\partial w}{\partial y}\right]\left(n_{y} m_{1 z}+n_{z} m_{1 y}\right)=0 \quad \text { em } \quad \partial \Omega_{4} .
\end{aligned}
$$

Mais detalhes sobre a descrição das equações na superfícies livre podem ser encontrados em Tomé e Mckee [163], para o caso bidimensional, e em Tomé et al. [155] para o caso tridimensional.

A simulação computacional de escoamentos de fluidos com superfícies livres, governados pelas equações (2.1) e (2.2), juntamente com as equações (2.7) e (2.8) (e (2.13) nos problemas tridimensionais) apresenta algumas dificuldades que despertaram, e ainda despertam muito interesse na comunidade científica. Uma dificuldade está relacionada à representação geométrica do domínio em movimento. Outra está relacionada à modelagem e solução numérica das equações governantes, respeitando as condições de contorno nas fronteiras rígidas e móveis. Neste trabalho, apenas a segunda dificuldade é investigada. Desta forma, utiliza-se uma formulação muito comum para resolver problemas envolvendo superfícies livres: uma malha fixa estacionária e um método de identificação para localizar onde a superfície livre encontra-se dentro da malha. Detalhes do esquema que segue essa formulação serão descrito a seguir, e serão utilizado ao longo deste trabalho. 


\subsection{Discretização temporal}

Uma etapa importante na simulação numérica de escoamentos transientes é a escolha do método de discretização temporal. As equações (2.1) e (2.2) podem ser discretizadas no tempo da seguinte forma

$$
\begin{gathered}
\frac{\mathbf{u}^{(n+1)}}{\delta t}-\theta_{1} R e^{-1} \nabla^{2} \mathbf{u}^{(n+1)}=\frac{\mathbf{u}^{(n)}}{\delta t}+\theta_{4} R e^{-1} \nabla^{2} \mathbf{u}^{(n)}-\theta_{2} \nabla \cdot(\mathbf{u u})^{(n)}+\theta_{3} \nabla \cdot(\mathbf{u u})^{(n-1)} \\
-\alpha \nabla p^{(n+1)}-(1-\alpha) \nabla p^{(n)}+\left(F r^{2}\right)^{-1} \mathbf{g}^{(n)} \\
\nabla \cdot \mathbf{u}^{(n+1)}=0
\end{gathered}
$$

onde os parâmetros $\theta_{1}, \theta_{2}, \theta_{3}$ e $\theta_{4}$ são determinados conforme o esquema de discretização temporal escolhido. Neste trabalho, utiliza-se o super-escrito $(n)$ para descrever o nível de tempo na discretização temporal. A tabela 2.1 apresenta os possíveis valores dos parâmetros.

Tabela 2.1: Esquemas de discretização temporal. Ex: método explícito, EI: método de Euler implícito, CN: método de Crank-Nicolson e AB/CN: método de Adams-Bashforth/CrankNicolson.

\begin{tabular}{|c|c|c|c|c|}
\hline Método & $\theta_{1}$ & $\theta_{2}$ & $\theta_{3}$ & $\theta_{4}$ \\
\hline $\mathrm{Ex}$ & 0 & 1 & 0 & 1 \\
\hline $\mathrm{EI}$ & 1 & 1 & 0 & 0 \\
\hline $\mathrm{CN}$ & 0.5 & 1 & 0 & 0.5 \\
\hline $\mathrm{AB} / \mathrm{CN}$ & 0.5 & 1.5 & 0.5 & 0.5 \\
\hline
\end{tabular}

Na equação (2.17), o esquema generalizado

$$
-\alpha \nabla p^{(n+1)}-(1-\alpha) \nabla p^{(n)}
$$

onde $\alpha \in(0,1]$, para a discretização da pressão é usado. Quando $\alpha=1$, um esquema de Euler implícito é empregado para a atualização da pressão. Para $\alpha=\frac{1}{2}$ o esquema utilizado é o trapezoidal (ou Crank-Nicolson), e a pressão na equação (2.17) é aproximada no nível de tempo $\left(n+\frac{1}{2}\right)$. 


\subsection{Malha computacional}

A estrutura de malha utilizada no presente trabalho é a malha deslocada (staggered grid), introduzida inicialmente por Harlow e Welch [66]. Este tipo de malha é muito utilizada no contexto dos métodos MAC pois possui algumas propriedades atrativas: é conhecida por garantir localmente a conservação de massa, movimento, energia cinética, e vorticidade [124]; é computacionalmente simples; é propícia para o uso de métodos de projeção no cálculo da pressão; e satisfaz a restrição de incompressibilidade [124].

No caso bidimensional, as velocidades horizontais $u$ são armazenadas nas arestas verticais, as velocidades verticais $v$ são armazenadas nas aresta horizontais, e quantidades escalares e demais grandezas $\chi$ são armazenadas no centro da célula. Para escoamentos tridimensionais utilizando a malha deslocada, as velocidades são armazenadas nas faces, enquanto que as quantidades escalares e outras grandezas são armazenadas no centro da célula. A figura 2.2a) ilustra uma célula no caso bidimensional, e a figura 2.2b) para o caso tridimensional.

a)

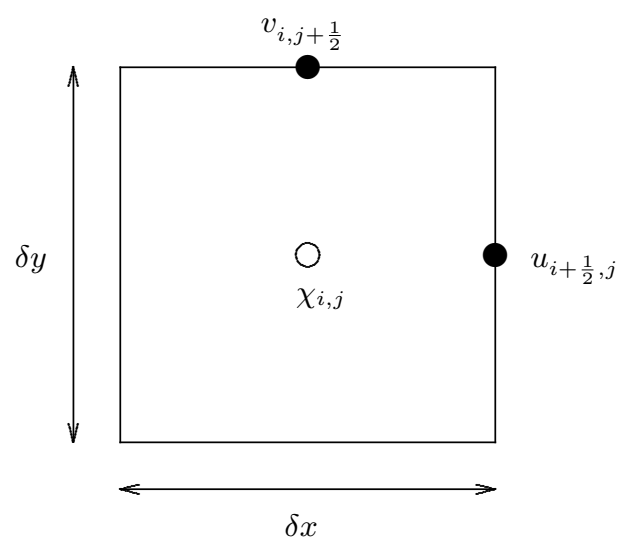

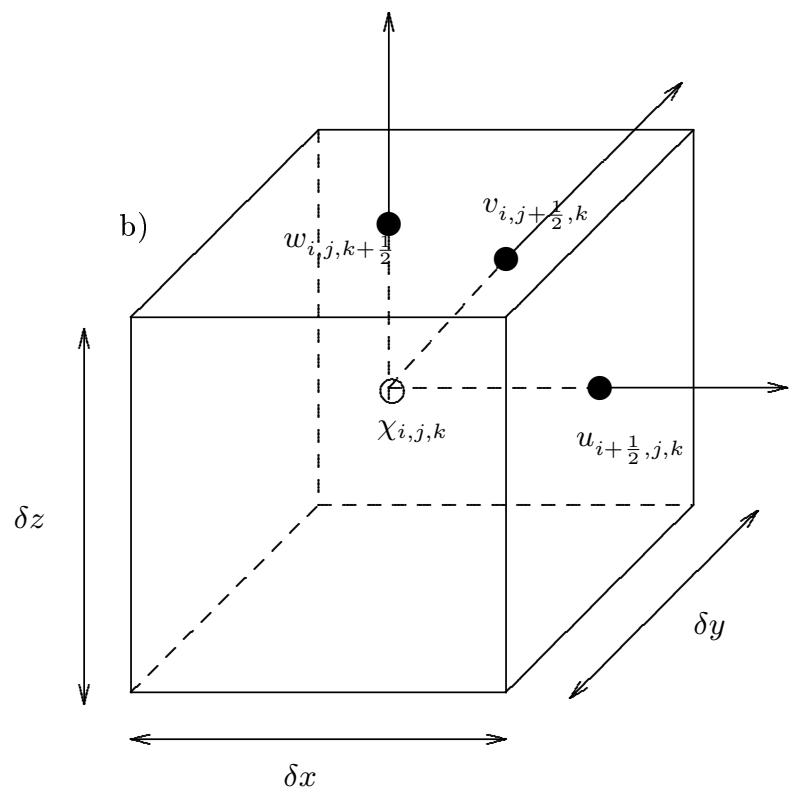

Figura 2.2: Exemplos de armazenamento em uma malha deslocada: a) caso bidimensional e b) caso tridimensional. 


\subsection{Partículas marcadoras e células}

A essência do método MAC são as partículas marcadoras e as células de uma malha euleriana. As partículas marcadoras são movidas de suas posições no tempo $t$ para novas posições no tempo posterior, de acordo as velocidades do sistema. A localização do fluido no interior da malha é determinado por um conjunto de partículas marcadoras que movem-se com o fluido. $\mathrm{O}$ sucesso do método MAC na solução de vários problemas de escoamentos com superfícies livres é bem documentado em diversas publicações. A razão para esse sucesso é que as partículas marcadoras não capturam diretamente as superfícies, sendo simplesmente as fronteiras de um volume de fluido. No trabalho de Nichols e Hirt [109], exemplos de vantagens e limitações do método MAC foram apresentadas.

No presente trabalho, baseado no método GENSMAC, as partículas marcadoras são mantidas apenas nas superfícies e não em todo domínio, como no método MAC. Esta estratégia visa economizar memória e tempo de processamento nas simulações numéricas. Usando essas partículas, a superfície livre é aproximada por uma superfície linear e representada por uma estrutura chamada halfedge [106]. Entre algumas vantagens da aproximação linear estão a capacidade de manter um espaçamento uniforme das partículas e as simplificações nos cálculos de intersecções entre superfícies diferentes. Mais detalhes sobre as implementações das estruturas utilizadas para representar a superfície livre podem ser encontrados em [15].

As propriedades do escoamento são representadas em uma malha de células retangulares (no caso bidimensional) classificadas a seguir. Células de contorno [B](Boundary) definem o contorno rígido; células do injetor $[\mathbf{I}]($ Inflow) definem a entrada de fluido, enquanto que células do ejetor $[\mathbf{O}]($ Outflow) definem a saída de fluido no domínio. Células vazias $[\mathbf{E}]($ Empty) são células que não contém o fluido de interesse. Células de superfície livre [S](Surface) são células que contém fluido e têm no mínimo uma face em contato com células vazias. Células cheias $[\mathbf{F}]($ Full $)$ denotam células cheias de fluido; nenhuma célula $[\mathbf{F}]$ pode ter faces em contato com células do tipo $[\mathbf{E}]$.

A figura 2.3 mostra um exemplo de estrutura de células de um escoamento em um dado tempo comparado com uma foto de um escoamento real; a classificação de células é baseada no problema físico. Para tornar a ilustração mais clara, as células vazias não são marcadas.

A classificação das células é atualizada a cada passo temporal utilizando informações fornecidas pelas partículas marcadoras. Portanto, é necessário apenas verificar a classificação de células na vizinhança das células [S]. Esta atualização é feita de acordo com os seguintes passos (veja em Castelo et al. [15]): 

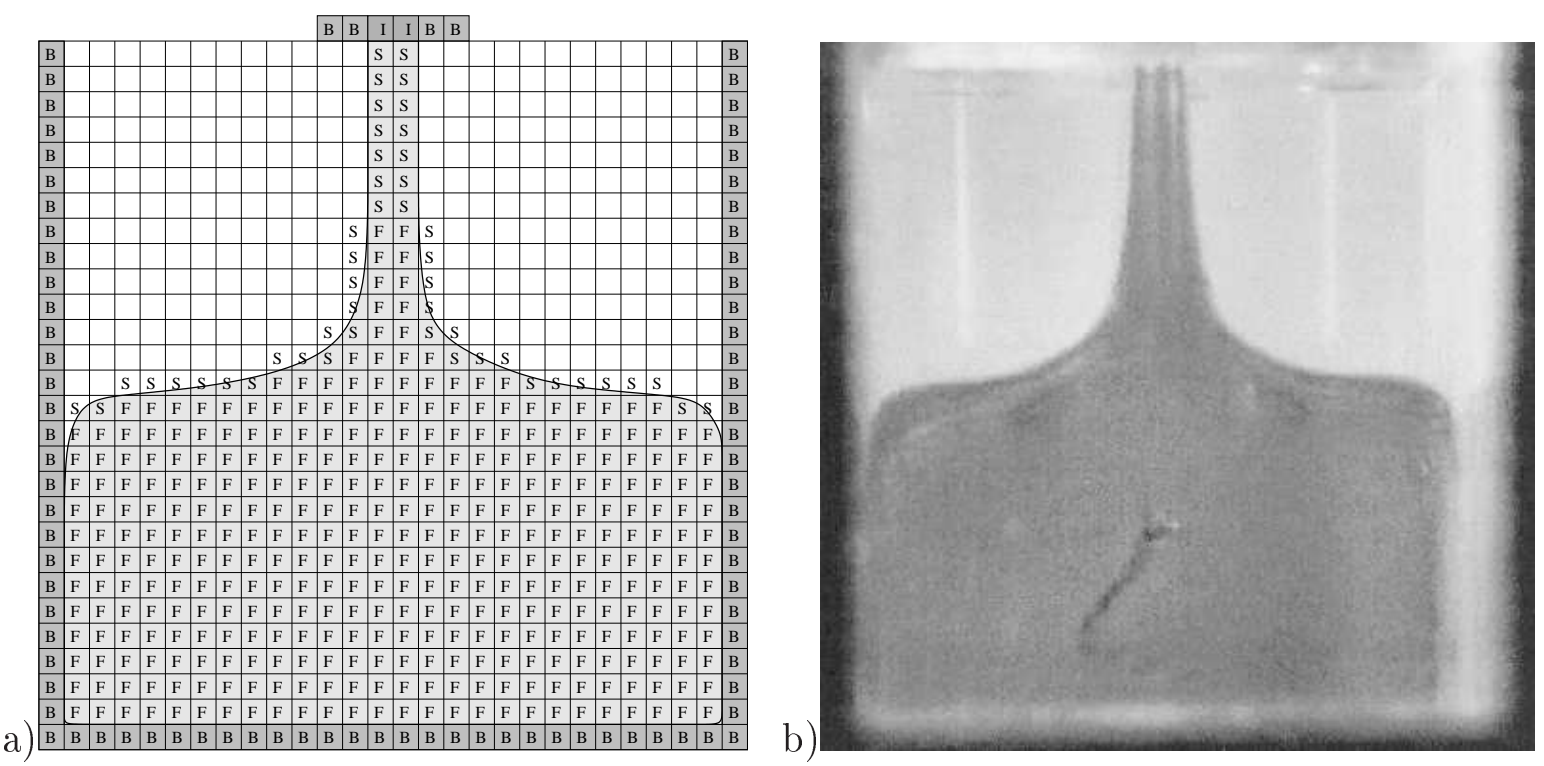

Figura 2.3: a) Classificação de células. b) Visualização de um escoamento de xarope de glicose [164].

1. As células que contém partículas são identificadas. Se alguma dessas células são do tipo $[\mathbf{E}]$, então no próximo passo elas são marcadas como células $[\mathbf{S}]$;

2. Células marcadas como $[\mathbf{S}]$, que não contém partículas são reescritas como células $[\mathbf{E}]$ ou [F], dependendo da classificação das células vizinhas;

3. As células $[\mathbf{F}]$ que contém partículas e têm alguma célula vizinha do tipo $[\mathbf{E}]$ são marcadas como $[\mathbf{S}]$.

A restrição imposta ao passo temporal para a classificação das células será apresentada na subseção 2.7.2.

\subsection{Aproximações da superfície livre}

Para aplicar as condições de contorno na superfície livre, utiliza-se as idéias propostas por Tomé e McKee em [163] para as equações (2.11) e (2.12) no caso bidimensional, e por Tomé et al. em [155] para as equações (2.14), (2.15) e (2.16) no caso tridimensional. A seguir uma breve descrição das aproximações realizadas na superfície livre para este trabalho. 


\subsubsection{Caso bidimensional}

As equações (2.11) e (2.12) são aproximadas considerando três tipos de orientação para a superfície livre: vertical (ver figura 2.4), horizontal (ver figura 2.5) e inclinada de $45^{\circ}$ (ver figura 2.6). Esta orientação é determinada de acordo com o vetor normal $\mathbf{n}$ no centro da célula [S] em questão.
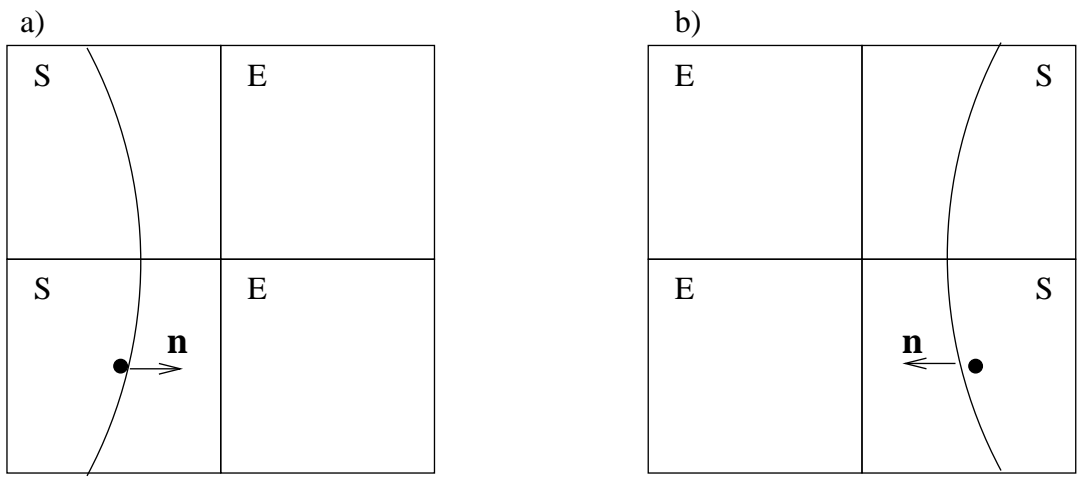

Figura 2.4: Orientação vertical determinada pelos vetores: a) $\mathbf{n}=(1,0)$ ou b) $\mathbf{n}=(-1,0)$.
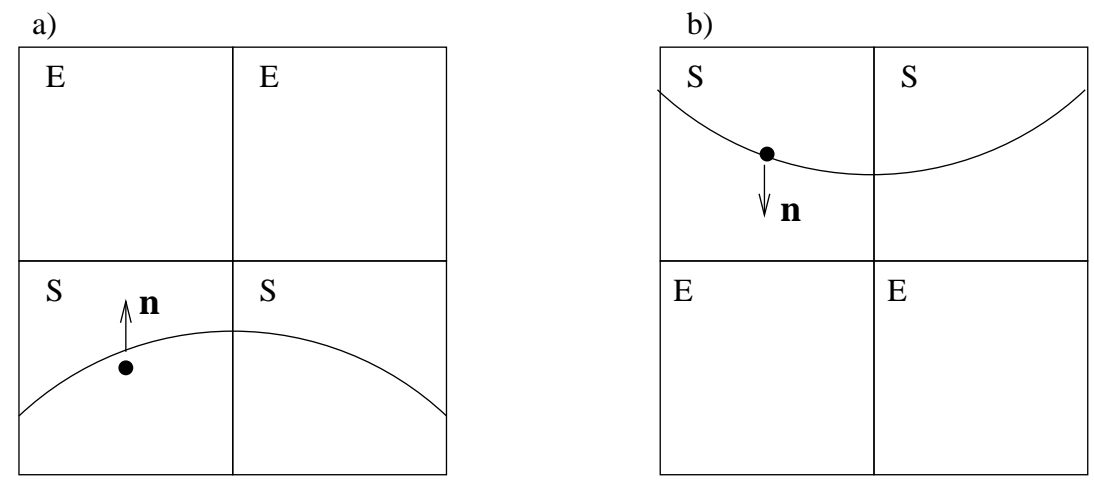

Figura 2.5: Orientação horizontal determinada pelos vetores: a) $\mathbf{n}=(0,1)$ ou b) $\mathbf{n}=(0,-1)$.

Assumindo que as dimensões das células são pequenas o suficiente para que a superfície intercepte uma célula em apenas dois lados, obtém-se três casos possíveis para analisar. Estes casos são determinados de acordo com a localização das células $[\mathbf{S}]$ e [E].

- Caso 1: A célula [S] tem uma face (direita, esquerda, inferior ou superior) em contato com a célula $[\mathbf{E}]$. Para este caso assume-se que a superfície será vertical ou horizontal, como por exemplo, nas figuras 2.4 e 2.5; 

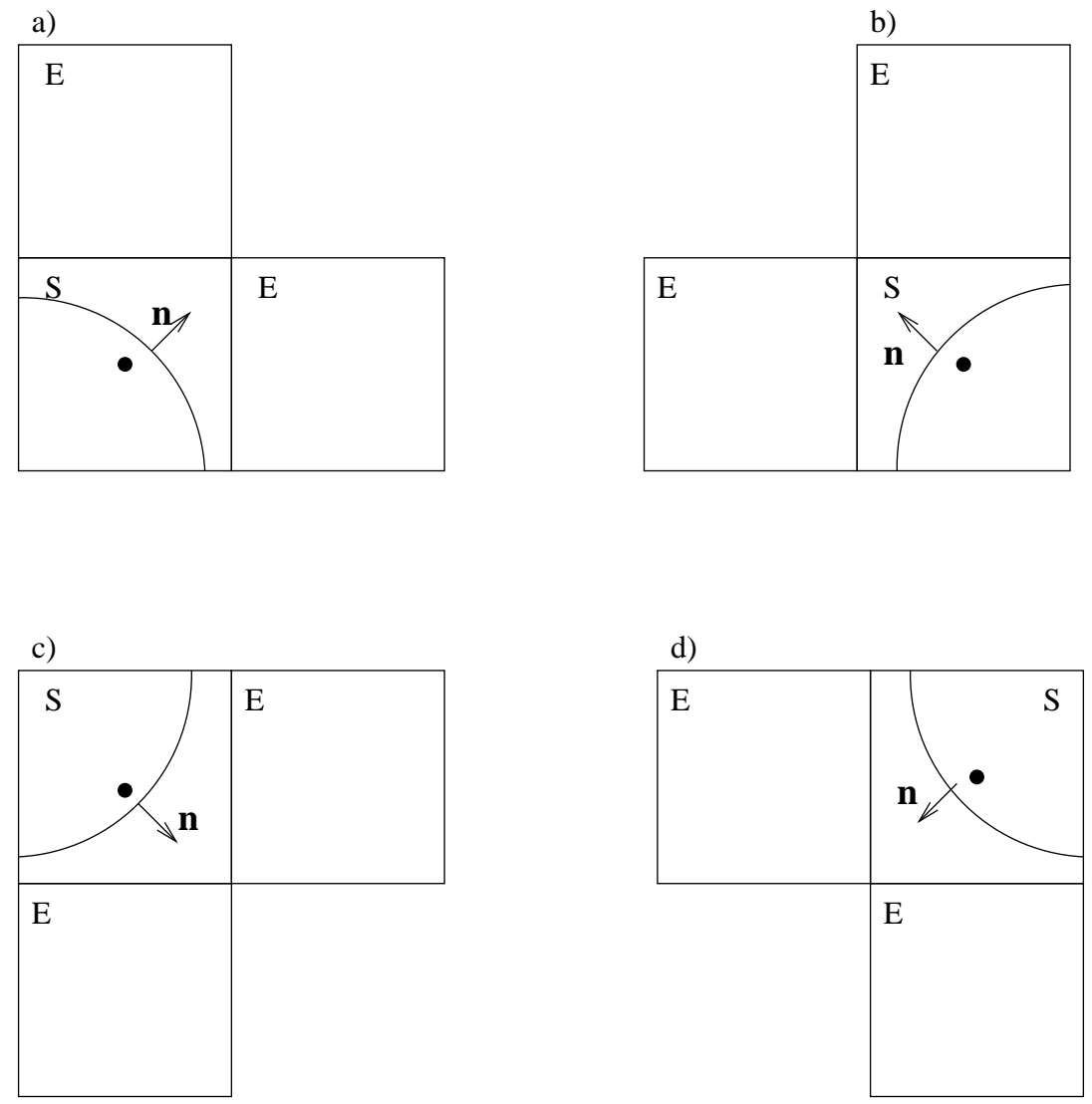

Figura 2.6: Orientação inclinada determinada pelos vetores: a) $\left.\mathbf{n}=\left(\frac{\sqrt{2}}{2}, \frac{\sqrt{2}}{2}\right), b\right) \mathbf{n}=\left(-\frac{\sqrt{2}}{2}, \frac{\sqrt{2}}{2}\right)$, c) $\mathbf{n}=\left(\frac{\sqrt{2}}{2},-\frac{\sqrt{2}}{2}\right)$ e d) $\mathbf{n}=\left(-\frac{\sqrt{2}}{2},-\frac{\sqrt{2}}{2}\right)$.

- Caso 2: Neste caso, uma célula $[\mathbf{S}]$ tem apenas duas faces em contato com células [E], e admite-se que o vetor normal forma um anglo de $45^{\circ} \mathrm{com}$ as faces em comum com a célula $[\mathbf{E}]$. A figura 2.6 apresenta os possíveis casos para o vetor $\mathbf{n}$;

- Caso 3: Este caso é especial, pois a célula [S] tem três lados em contato com células $[\mathbf{E}]$ ou duas faces opostas em contato com células $[\mathbf{E}]$. Quando isto acontece, o valor da quantidade escalar no centro é definida como zero, e as velocidades são ajustadas. Para evitar tal problema, o refinamento da malha pode ser uma solução. 


\subsubsection{Caso tridimensional}

Neste caso, para aplicar as equações $(2.14),(2.15)$ e (2.16) a superfície livre pode ser aproximada por uma superfície plana. As superfícies são definidas como: superfície planar paralela aos eixos coordenados (ver figura 2.7), superfície planar inclinada de $45^{\circ}$ (ver figura 2.8) e superfície planar inclinada de $60^{\circ}$ (ver figura 2.9).

Análogo ao caso bidimensional, assumindo que o espaçamento de malha é suficientemente pequeno para que a superfície seja aproximada por uma superfície planar, obtém-se quatro casos possíveis para analisar. Estes casos são determinados de acordo com a localização das células $[\mathbf{S}]$ e $[\mathbf{E}]$.

- Caso 1: A célula de superfície livre [S] tem apenas uma face (direita, esquerda, frontal, traseira, inferior ou superior) em contato com a célula vazia [E]. Assume-se que o vetor normal aponta para a direção de um dos eixos coordenados. Logo, o vetor normal unitário pode ser definido como $( \pm 1,0,0)$ ou $(0, \pm 1,0)$ ou $(0,0, \pm 1)$. Um exemplo para este caso está ilustrado na figura 2.7, na qual o vetor normal está apontando para a célula $[\mathbf{E}]$ com $\mathbf{n}=(0,0,1)$.

- Caso 2: As células [S] possuem duas faces adjacentes em contato com faces de células vazias $[\mathbf{E}]$. Assim, admite-se que o vetor normal unitário pode ser tomado como $\mathbf{n}=$ $\left( \pm \frac{\sqrt{2}}{2}, \pm \frac{\sqrt{2}}{2}, 0\right)$ ou $\mathbf{n}=\left( \pm \frac{\sqrt{2}}{2}, 0, \pm \frac{\sqrt{2}}{2}\right)$ ou $\mathbf{n}=\left(0, \pm \frac{\sqrt{2}}{2}, \pm \frac{\sqrt{2}}{2}\right)$. Na figura 2.8, apresenta-se uma célula $[\mathbf{S}] \operatorname{com} \mathbf{n}=\left(\frac{\sqrt{2}}{2}, 0, \frac{\sqrt{2}}{2}\right)$.

- Caso 3: As células [S] possuem três faces adjacentes em contato com faces de células vazias $[\mathbf{E}]$. Nestas configurações, o vetor normal unitário faz um ângulo de $60^{\circ}$ com os eixos coordenados e pode ser descrito como $\mathbf{n}=\left( \pm \frac{\sqrt{3}}{3}, \pm \frac{\sqrt{3}}{3}, \pm \frac{\sqrt{3}}{3}\right)$. A figura 2.9 ilustra o caso onde $\mathbf{n}=\left(\frac{\sqrt{3}}{3},-\frac{\sqrt{3}}{3}, \frac{\sqrt{3}}{3}\right)$.

- Caso 4: Quando uma célula de superfície [S] tem no mínimo duas faces opostas em contato com células [E], não é possível obter informações sobre o vetor normal. Nestes casos, analogamente ao caso bidimensional, o valor da quantidade escalar no centro é definida como zero, e as velocidades são ajustadas para que a massa seja conservada. Para evitar tal problema, assim como sugerido anteriormente no caso bidimensional, o refinamento da malha pode ser uma solução. 


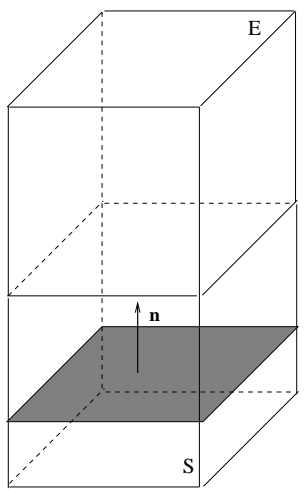

Figura 2.7: Exemplo de uma superfície planar paralela ao plano formado pelos eixos $x y$. Em particular, neste caso $\mathbf{n}=(0,0,1)$.

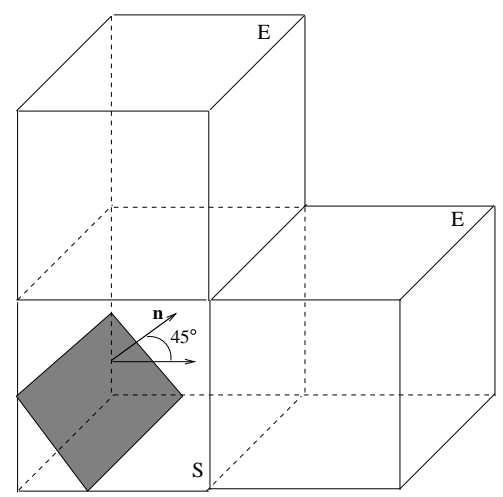

Figura 2.8: Exemplo de uma superfície planar inclinada de $45^{\circ}$. Em particular, neste caso $\mathbf{n}=\left(\frac{\sqrt{2}}{2}, 0, \frac{\sqrt{2}}{2}\right)$.

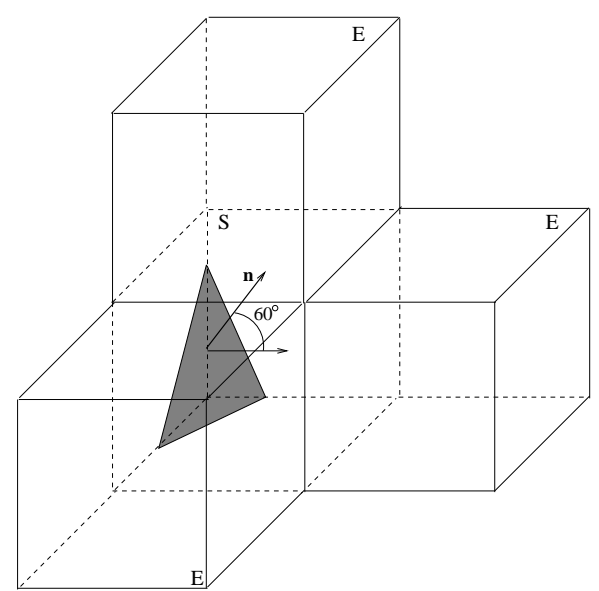

Figura 2.9: Exemplo de uma superfície planar inclinada de $60^{\circ}$. Em particular, neste caso $\mathbf{n}=\left(\frac{\sqrt{3}}{3},-\frac{\sqrt{3}}{3}, \frac{\sqrt{3}}{3}\right)$. 


\subsection{Método de projeção}

Um fato significante na solução numérica das equações de Navier-Stokes é que o sistema de equações (2.1)-(2.2) acopla os campos de velocidade e pressão devido à restrição de incompressibilidade. A resolução numérica desse sistema acoplado é muitas vezes descartada na prática, pois métodos que utilizam essa estratégia apresentam dificuldades computacionais. Essa ineficiência é conseqüência da dificuldade na solução do sistema (2.1)-(2.2) que é extenso, e também devido às não-linearidades envolvidas no sistema. Desta forma, uma estratégia para contornar tais problemas é o desacoplamento das equações.

Os métodos que utilizam a estratégia do desacoplamento dos campos de velocidade e pressão das equações (2.1) e (2.2) foram originalmente propostos por Harlow e Welch [66], Chorin [22, 23], e Temam [152], conhecidos como "métodos de projeção" ou "métodos de passo fracionário". A formulação matemática dos métodos de projeção está relacionada com o teorema de Helmholtz-Hodge [72, 85] na decomposição de um campo vetorial em dimensão finita. O teorema é apresentado a seguir.

Teorema 1 (Decomposição de Helmholtz-Hodge (DHH)) : Seja $\Omega$ um dominio suave e limitado, e $\widetilde{\mathbf{u}}$ um campo vetorial suave em $\Omega$. O campo vetorial $\widetilde{\mathbf{u}}$ pode ser decomposto em

$$
\widetilde{\mathbf{u}}=\mathbf{u}+\nabla \phi,
$$

onde

$$
\nabla \cdot \mathbf{u}=0 \quad \text { em } \quad \Omega, \quad \mathbf{u} \cdot \mathbf{n}=U \quad \text { em } \quad \partial \Omega .
$$

Neste caso, $\mathbf{n}$ é o vetor unitário externo à fronteira $\partial \Omega, \mathbf{u}$ é um campo vetorial dado e $\phi$ uma função escalar.

A demonstração desse teorema pode ser encontrada em [35], e sua interpretação está ilustrada na figura 2.10. A seguir utiliza-se o teorema DHH na construção de um método de projeção.

A idéia básica por trás desses métodos é usar a equação de quantidade de movimento (2.1) para calcular um campo de velocidade intermediária, que geralmente não satisfaz a equação da continuidade (2.2), ou seja, define-se a equação

$$
\frac{\partial \widetilde{\mathbf{u}}}{\partial t}+\nabla \cdot(\mathbf{u u})=-\nabla \widetilde{p}+R e^{-1} \nabla^{2} \mathbf{u}+\left(F r^{2}\right)^{-1} \mathbf{g} \quad \text { em } \quad[0, T] \times \Omega
$$




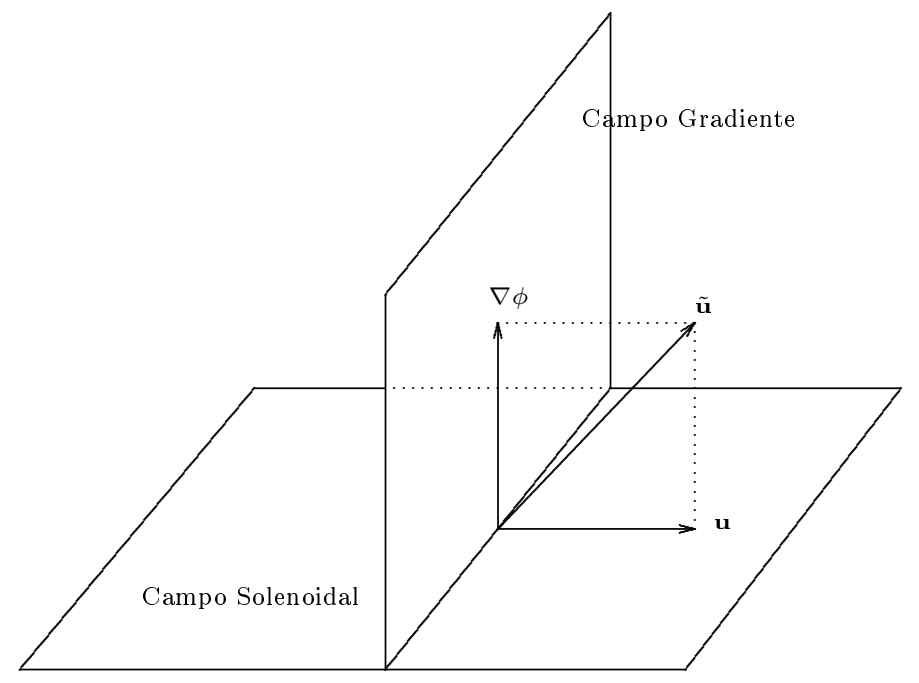

Figura 2.10: Ilustração da decomposição de um campo vetorial aplicando o teorema de Helmholtz-Hodge.

onde $\widetilde{p}$ é uma aproximação para a pressão (ou uma pressão provisória). Neste momento aplicase o teorema DHH, interpretando que a velocidade $\widetilde{\mathbf{u}}$ é o campo vetorial decomposto como em (2.20). Em muitos casos, a velocidade $\widetilde{\mathbf{u}}$ não é solenoidal, isto é, não satisfaz a equação da continuidade (2.2), pois em geral $p \neq \widetilde{p}$. A função da pressão em escoamentos incompressíveis é fazer com que o campo de velocidade satisfaça a equação da continuidade (2.2). Portanto é necessário calcular a pressão no próximo passo.

Para isto uma equação elíptica é resolvida para que a equação (2.2) seja satisfeita e a pressão determinada. A importância dessa equação elíptica para a pressão, é que ela faz a ligação entre as equações de quantidade de movimento e da continuidade. Para obter esta equação, novamente utiliza-se o teorema DHH, aplicando o divergente na equação (2.20), e em seguida utilizando a equação da continuidade (2.2) para definir

$$
\nabla^{2} \phi=\nabla \cdot \widetilde{\mathbf{u}}
$$

Após esse passo, utiliza-se a equação (2.20) para atualizar os campos de velocidade e de pressão. Portanto, a principal vantagem da classe de métodos tipo projeção é que os cálculos dos campos de velocidade e pressão estão desacoplados. Mais precisamente, assumindo que os termos não-lineares são tratados explicitamente, a cada passo no tempo resolve-se um conjunto de equações para a velocidade e uma equação escalar do tipo Poisson para a pressão. Esta estratégia utilizada pelos métodos de projeção é computacionalmente muito eficiente quando 
comparado com métodos de solução tipo acoplado, especialmente quando métodos iterativos eficientes são aplicados na solução da equação de Poisson.

Estudos rigorosos sobre a consistência e estabilidade dos métodos de projeção para escoamentos incompressíveis foram apresentados por Shen [140, 141], Guermond e seus co-autores [63, 61], e por Denaro [35]. Uma breve descrição de trabalhos relacionados a métodos de projeção para escoamentos incompressíveis será apresentada no capítulo 6 .

\subsection{Metodologia GENSMAC}

Nesta seção, uma breve descrição da metodologia GENSMAC será apresentada. O método GENSMAC descrito por Tomé e McKee [163] é uma extensão do método SMAC [4] para domínios bidimensionais arbitrários. O método GENSMAC utiliza uma variante do método de projeção descrito anteriormente para sua formulação matemática. O procedimento de solução do método GENSMAC será apresentado a seguir.

Inicialmente, considera-se a discretização explícita da equação de quantidade de movimento (2.17) de acordo com a tabela 2.1, ou seja, considera-se a seguinte equação discreta no tempo

$$
\frac{\mathbf{u}^{(n+1)}}{\delta t}=\frac{\mathbf{u}^{(n)}}{\delta t}+R e^{-1} \nabla^{2} \mathbf{u}^{(n)}-\nabla \cdot(\mathbf{u u})^{(n)}-\nabla p^{(n+1)}+\left(F r^{2}\right)^{-1} \mathbf{g}^{(n)}
$$

onde um esquema de Euler implícito é empregado para a atualização da pressão (assume-se $\alpha=1$ na equação (2.19)). Como descrito anteriormente, a idéia dos métodos de projeção é utilizar a equação de quantidade de movimento e resolvê-la para um campo de velocidade intermediário. Logo, a equação (2.24) é reescrita como

$$
\frac{\widetilde{\mathbf{u}}}{\delta t}=\frac{\mathbf{u}^{(n)}}{\delta t}+R e^{-1} \nabla^{2} \mathbf{u}^{(n)}-\nabla \cdot(\mathbf{u u})^{(n)}-\nabla p^{(n)}+\left(F r^{2}\right)^{-1} \mathbf{g}^{(n)},
$$

onde $p^{(n)}=\widetilde{p}$ é uma aproximação para a pressão $p^{(n+1)}$. Por simplicidade de notação, a partir deste momento utiliza-se $p^{(n)}$ no lugar de $\widetilde{p}$.

As condições de contorno em paredes rígidas para $\widetilde{\mathbf{u}}$ na equação (2.25) são

$$
\begin{aligned}
& \widetilde{\mathbf{u}} \cdot \mathbf{n}=\mathbf{u}^{(n+1)} \cdot \mathbf{n} \quad \text { em } \quad \partial \Omega_{1}, \\
& \widetilde{\mathbf{u}} \cdot \mathbf{m}=\mathbf{u}^{(n+1)} \cdot \mathbf{m} \quad \text { em } \quad \partial \Omega_{1} .
\end{aligned}
$$


Ou seja, as condições de contorno para $\widetilde{\mathbf{u}}$ são as mesmas impostas para $\mathbf{u}^{(n+1)}$ em (2.4) em problemas de contorno sem deslizamento e (2.5) em contorno sem aderência. Também impõemse as mesmas condições de contorno de $\mathbf{u}^{(n+1)}$ em $\widetilde{\mathbf{u}}$ para os contornos do tipo injetor $\partial \Omega_{2}$ e ejetor $\partial \Omega_{3}$ (ver equação (2.6)).

Utilizando a equação (2.20), o campo de velocidade pode ser decomposto como

$$
\mathbf{u}^{(n+1)}=\widetilde{\mathbf{u}}-\delta t \nabla \psi^{(n+1)}
$$

onde $\phi^{(n+1)}=\delta t \psi^{(n+1)}$.

Aplicando o operador divergente na equação (2.27) e impondo a conservação de massa para $\mathbf{u}^{(n+1)}$ tem-se uma equação de Poisson para uma pressão tentativa (ou provisória) $\psi^{(n+1)}$, dada por

$$
\nabla^{2} \psi^{(n+1)}=\frac{1}{\delta t} \nabla \cdot \widetilde{\mathbf{u}}
$$

De acordo com Tomé e McKee [163], as condições de contorno impostas para $\psi$ nesta equação são

$$
\frac{\partial \psi}{\partial n}=0 \quad \text { em } \quad \partial \Omega_{1} \quad \text { e } \quad \partial \Omega_{2}
$$

e

$$
\psi=0 \quad \text { em } \quad \partial \Omega_{3} \quad \text { e } \quad \partial \Omega_{4} .
$$

Ou seja, na metodologia GENSMAC impõem-se condições homogêneas do tipo Neumann em contorno de parede rígida e injetor e condições homogêneas do tipo Dirichlet no ejetor e na superfície livre.

Para obter uma equação para o campo final da pressão, substitui-se (2.27) em (2.25), ou seja,

$$
\frac{\mathbf{u}^{(n+1)}+\delta t \nabla \psi^{(n+1)}}{\delta t}=\frac{\mathbf{u}^{(n)}}{\delta t}+R e^{-1} \nabla^{2} \mathbf{u}^{(n)}-\nabla \cdot(\mathbf{u u})^{(n)}-\nabla p^{(n)}+\left(F r^{2}\right)^{-1} \mathbf{g}^{(n)} .
$$

Quando (2.31) é comparada com (2.24), obtém-se

$$
p^{(n+1)}=p^{(n)}+\psi^{(n+1)} .
$$




\subsubsection{Algoritmo do método GENSMAC}

Utilizando as equações discretas no tempo, os passos do algoritmo do método GENSMAC podem ser descritos resumidamente como

- Passo 1: Seja $p^{(n)}=\widetilde{p}$ um campo de pressão que satisfaz as condições de contorno na superfície livre. Esse campo de pressão é calculado de acordo com a equação (2.7) e as velocidades nas superfícies livres por (2.8) (adicionando (2.13) em problemas tridimensionais).

- Passo 2: Conhecido o campo de pressão calcular um campo de velocidade intermediário $\widetilde{\mathbf{u}}$ em $t_{n+1}=t_{n}+\delta t$ satisfazendo a equação (2.25).

- Passo 3: Resolver a equação de Poisson (2.28) com as condições de contorno (2.29) e $(2.30)$.

- Passo 4: Atualizar o campo de velocidade final pela equação (2.27).

- Passo 5: Atualizar o campo de pressão final pela equação (2.32).

- Passo 6: Determinar as novas posições das partículas marcadoras virtuais resolvendo

$$
\dot{\mathbf{x}}=\mathbf{u}^{(n+1)},
$$

pelo método de Euler explícito. Detalhes sobre a movimentação das partículas marcadoras, realizada neste passo, podem ser encontrados em Castelo et al. [15].

Os passos acima formam um ciclo computacional, a partir de um tempo inicial $t_{n}$, para o cálculo das variáveis primitivas em um tempo posterior $t_{n+1}=t_{n}+\delta t$.

\subsubsection{Restrição sobre o passo temporal}

Quando um método explícito é aplicado para resolver a equação de quantidade de movimento, isto é, escolhendo $\theta_{2}=\theta_{4}=1$ e $\theta_{1}=\theta_{3}=0$ em (2.17), uma restrição de estabilidade parabólica é necessária. Essa restrição, no caso bidimensional é dada pela expressão

$$
\delta t_{v i s c} \leq \frac{R e}{2}\left(\frac{1}{(\delta x)^{2}}+\frac{1}{(\delta y)^{2}}\right)^{-1}
$$


onde $\delta t_{v i s c}$ é o passo temporal adimensional resultante da condição de estabilidade nos termos viscosos, e $\delta x$ e $\delta y$ são os espaçamentos adimensionais de malha nas direções $x$ e $y$ respectivamente. Analogamente, pode-se determinar esta restrição parabólica no caso tridimensional, produzindo

$$
\delta t_{v i s c} \leq \frac{R e}{2}\left(\frac{1}{(\delta x)^{2}}+\frac{1}{(\delta y)^{2}}+\frac{1}{(\delta z)^{2}}\right)^{-1},
$$

onde $\delta z$ é o espaçamento de malha na direção $z$.

O passo temporal do método GENSMAC, por utilizar uma formulação explícita para resolver (2.25), é calculado de acordo com as expressões (2.34) em problemas bidimensionais, e (2.35) para problemas tridimensionais. Em princípio, quando uma discretização temporal implícita é aplicada à equação (2.17), as restrições parabólicas não se aplicam. Porém, tanto para formulação explícita, quanto para formulação implícita, em escoamentos com superfícies livres outra restrição é necessária.

Quando o número de Reynolds é bem menor do que um, esta restrição é menos exigente do que a restrição parabólica, pois deriva um passo temporal maior. Baseada no fato de que uma partícula de fluido não pode percorrer, em cada passo de tempo, uma distância maior do que o tamanho de uma célula, as seguintes restrições no passo temporal são derivadas:

$$
\delta t_{C F L x} \leq\left(\frac{\delta x}{|u|_{\max }}\right) \quad \text { e } \quad \delta t_{C F L y} \leq\left(\frac{\delta y}{|v|_{\max }}\right)
$$

onde $|u|_{\text {max }}$ e $|v|_{\text {max }}$ são módulos máximos das velocidades nas direções $x$ e $y$, respectivamente. Essas restrições são conhecidas na literatura como "condição Courant-Friedrichs-Lewy (CFL)" [27]. No caso tridimensional, além das inequações (2.36), deve-se também computar a restrição na direção $z$.

Desde que o movimento das partículas marcadoras é condicionado pela restrição CFL, as interfaces podem se mover no máximo uma célula a cada passo temporal.

\subsection{Esquemas implícitos na metodologia GENSMAC}

Em [113, 114] uma modificação na metodologia GENSMAC foi proposta. Os autores combinaram esquemas implícitos para tratar os termos difusivos e esquemas explícitos para tratar os termos convectivos da equação (2.22). Esta metodologia foi denominada pelos autores como GENSMAC semi-implícito. Para isso, os autores utilizaram os parâmetros da tabela 2.1, apre- 
sentada anteriormente, e testaram diferentes métodos implícitos na discretização temporal para a equação de quantidade de movimento. A justificativa para estas modificações, juntamente com outras técnicas implícitas para métodos de projeção para escoamentos com superfície livre serão apresentadas nos próximos capítulos.

Apenas como ilustração, nesta seção apresenta-se a aplicação dos métodos Euler implícito e Crank-Nicolson na equação para cálculo da velocidade intermediária (2.22). No caso do método de Euler implícito, de acordo com a tabela 2.1, tem-se

$$
\frac{\widetilde{\mathbf{u}}}{\delta t}-R e^{-1} \nabla^{2} \widetilde{\mathbf{u}}=\frac{\mathbf{u}^{(n)}}{\delta t}-\nabla \cdot(\mathbf{u u})^{(n)}-\nabla p^{(n)}+\left(F r^{2}\right)^{-1} \mathbf{g}^{(n)}
$$

e, para o esquema Crank-Nicolson obtém-se

$$
\frac{\widetilde{\mathbf{u}}}{\delta t}-\frac{1}{2 R e} \nabla^{2} \widetilde{\mathbf{u}}=\frac{\mathbf{u}^{(n)}}{\delta t}+\frac{1}{2 R e} \nabla^{2} \mathbf{u}^{(n)}-\nabla \cdot(\mathbf{u u})^{(n)}-\nabla p^{(n)}+\left(F r^{2}\right)^{-1} \mathbf{g}^{(n)},
$$

onde, em ambas equações, $p^{(n)}$ é uma aproximação para a pressão $p^{(n+1)}$.

Portanto, para aplicar os esquemas implícitos na metodologia GENSMAC, basta resolver as equações $(2.37)$ e/ou (2.38) no passo 2 do algoritmo apresentado na subseção 2.7.1. As condições de contorno na solução destas equações são as mesmas da equação (2.25). Os sistemas lineares resultantes da discretização das equações (2.37) e (2.38) são esparsos e simétricos, e são resolvidos pelo método dos gradientes conjugados [142]. 


\section{Estabilidade de métodos numéricos em malha deslocada}

Antes de iniciar este capítulo, uma pergunta é proposta ao leitor: "O método de CrankNicolson aplicado na equação do calor com qualquer tipo de condições de fronteira será sempre estável ?" Em particular, pode-se re-frasear esta questão para que se torne mais específica: "Quando que o método de Crank-Nicolson aplicado a equação unidimensional do calor sobre condições de contorno do tipo Dirichlet pode-se tornar condicionalmente estável?" Este capítulo tem como objetivo principal responder esta questão.

\subsection{Introdução}

O método MAC [66] foi um dos primeiros esquemas propostos para a solução de escoamentos viscosos, incompressíveis e transientes com superfície livre. Como comentado anteriormente, após o desenvolvimento do método MAC diversas extensões desse método foram, e ainda são, propostas na literatura. Duas características comuns nas técnicas baseadas no esquema MAC são a discretização explícita da equação de quantidade de movimento pelo método de Euler explícito e o uso de uma malha deslocada.

A principal vantagem da discretização explícita é a facilidade na implementação computacional, pois quando o método de Euler explícito é aplicado à equação de quantidade de movimento, este fornece um conjunto independente de equações que pode ser facilmente resolvido. O uso de 
uma malha deslocada apresenta duas vantagens importantes. Para uma célula computacional (como na figura 2.2) a equação discreta da continuidade utiliza a diferença de componentes de velocidades adjacentes, fato que impedirá o surgimento de um campo de velocidade instável [122]. A segunda vantagem de uma malha deslocada é permitir a utilização de discretizações de segunda ordem para pressão $p$ sem o risco de encontrar campos oscilatórios para a pressão, que ocorrem quando uma discretização de segunda ordem para $p$ é aplicada sobre uma malha co-localizada. Detalhes sobre as diferenças entre malhas deslocadas e co-localizadas para as equações de Navier-Stokes podem ser encontrados em [122, 50, 52].

Entretanto, algumas desvantagens aparecem no uso de métodos explícitos e da malha deslocada. O uso do método explícito implica que a restrição de estabilidade parabólica deverá ser respeitada. Essa restrição depende do número de Reynolds e dos espaçamentos de malha, e é dada pela expressão (2.34) no caso bidimensional, e por (2.35) no caso tridimensional. Para escoamentos em que as forças inerciais são superiores às forças viscosas, ou seja, quando $R e>1$, a condição de estabilidade não impõe uma restrição severa no passo temporal, e métodos explícitos produzirão uma solução numérica em tempo compatível. Entretanto, em algumas aplicações envolvendo fluidos newtonianos e não-newtonianos, escoamentos a baixo número de Reynolds são frequentes (por exemplo as aplicações em [162, 161]). Números de Reynolds da ordem de $10^{-1}$ a $10^{-4}$ são facilmente encontrados em aplicações envolvendo o escoamento de um polímero, como no inchamento do extrudado [74], enchimento de moldes [89], escoamentos em jatos [164], preenchimento de container [164], entre outras aplicações. Nesta classe de problemas, a aplicação de esquemas explícitos leva a um passo temporal muito pequeno, e conseqüentemente, o tempo de processamento torna-se inviável do ponto de vista computacional. Um exemplo destes esquemas é o método GENSMAC [163], que utiliza uma formulação explícita na discretização dos termos viscosos da equação de quantidade de movimento, e portanto seu passo temporal depende da restrição parabólica. Logo, este método requer um número elevado de ciclos computacionais e, como resultado, consome tempo de processamento elevado em simulações onde o valor do número de Reynolds é baixo $(R e<<1)$. Ou ainda, quando o refinamento da malha é necessário para se obter uma solução numérica melhor, grande quantidade de memória é utilizada. Portanto, com o objetivo de superar estes problemas, a aplicação de técnicas implícitas deve ser considerada.

Discretizações implícitas da equação de quantidade de movimento utilizando métodos conhecidos podem ser derivadas por analogia, como foi apresentado para os esquemas de Euler implícito e de Crank-Nicolson no capítulo 2. Entretanto, o uso de métodos implícitos para a equação de quantidade de movimento não garante que a formulação será incondicionalmente 
estável. Diversos pesquisadores têm se dedicado ao estudo de estabilidade destes esquemas implícitos para as equações de Navier-Stokes. Geralmente, estes estudos têm sido direcionado usando uma análise do tipo von Neumann (análise de Fourier) [113], no qual o efeito das condições de contorno na estabilidade da solução pode ser ignorado ou removido. Kwok e Tam [84] apresentaram uma análise linearizada do tipo von Neumann para vários esquemas de diferenças finitas em uma malha deslocada. Entretanto, neste trabalho, o efeito das condições de contorno na estabilidade dos métodos não foi discutido. Turek [169] realizou um estudo de estabilidade em diferentes esquemas de discretização implícita para a solução das equações de Navier-Stokes incompressível. Petersson [127] analisou a estabilidade do esquema de Euler implícito para diferentes condições de contorno para a pressão em escoamentos incompressíveis. Uma análise de estabilidade numérica para escoamentos em um cilindro foi apresentada por Prudhomme e Oden [131]. Outros estudos recentes têm sido realizados na estabilidade de esquemas implícitos aplicados às equações de Navier-Stokes: para fórmulas de diferenças atrasadas (backward differentiation formulas) [83], para uma discretização totalmente implícita das equações de Navier-Stokes utilizando o método de Euler implícito [166], e para esquemas de Runge-Kutta [111].

Um fato interessante na aplicação de métodos implícitos em escoamentos de fluidos foi apresentado por Oishi et al. [114, 115]. Nestes trabalhos, o esquema de Crank-Nicolson tornouse condicionalmente estável em malhas do tipo deslocada. Os autores acreditavam, que a instabilidade seria conseqüência das oscilações provocadas pelo comportamento assintótico da solução numérica que este método possui. Este fato foi brevemente discutido em [113] e bem discutido em [69, 169]. No entanto, nos trabalhos de Oishi et al. [114, 115], não foi realizado um estudo teórico para comprovar as suspeitas dos autores.

Com o objetivo de investigar o problema de instabilidade descrito em [114, 115], neste capítulo, apresenta-se um estudo de como as condições de contorno associadas às paredes rígidas e a malha deslocada influenciam na estabilidade de métodos implícitos. Como de costume na literatura em estudos de estabilidade, a análise é empreendida para um problema modelo: a equação do calor unidimensional com condições de contorno do tipo Dirichlet. Desta extensiva análise teórica, mostra-se como a substituição de condições de contorno implícitas por condições de contorno explícitas pode afetar profundamente o intervalo de estabilidade do método. 


\subsection{Problema modelo e discretização}

Antes de introduzir o estudo da estabilidade numérica de métodos aplicados a uma malha deslocada, considere o seguinte problema: a simulação numérica de um escoamento newtoniano incompressível transiente governado pelas equações de Navier-Stokes (2.1) e (2.2) em um canal vertical. As paredes laterais deste canal são rígidas e sem deslizamento, ou seja, aplica-se as condições de contorno do tipo no-slip ou sem escorregamento (2.4) . Utiliza-se um método de projeção (como descrito na seção 2.7), com diferentes métodos de discretização temporal para a solução da equação de quantidade de movimento (2.22). Como por exemplo, o esquema de Euler explícito (2.25), Euler implícito (2.37) e Crank-Nicolson (2.38). A malha considerada é a deslocada, como apresentada na figura 2.2a), e a classificação de célula utilizada é a mesma do GENSMAC, descrita na seção 2.4. Agora, considere uma faixa horizontal deste canal com espessura de uma célula e com $m+2$ células de comprimento, ou seja, uma célula na direção $y$, e $m+2$ células na direção $x$. Uma representação geométrica deste canal pode ser visto na figura 3.1. Com o objetivo de investigar a interferência das condições de contorno na estabilidade dos esquemas citados acima para solução da equação de quantidade de movimento (2.22), o problema bidimensional descrito pode ser representado por um problema modelo unidimensional, que apresente propriedades semelhantes, tais como a malha, condições de contorno, esquemas de discretização temporal, entre outras.

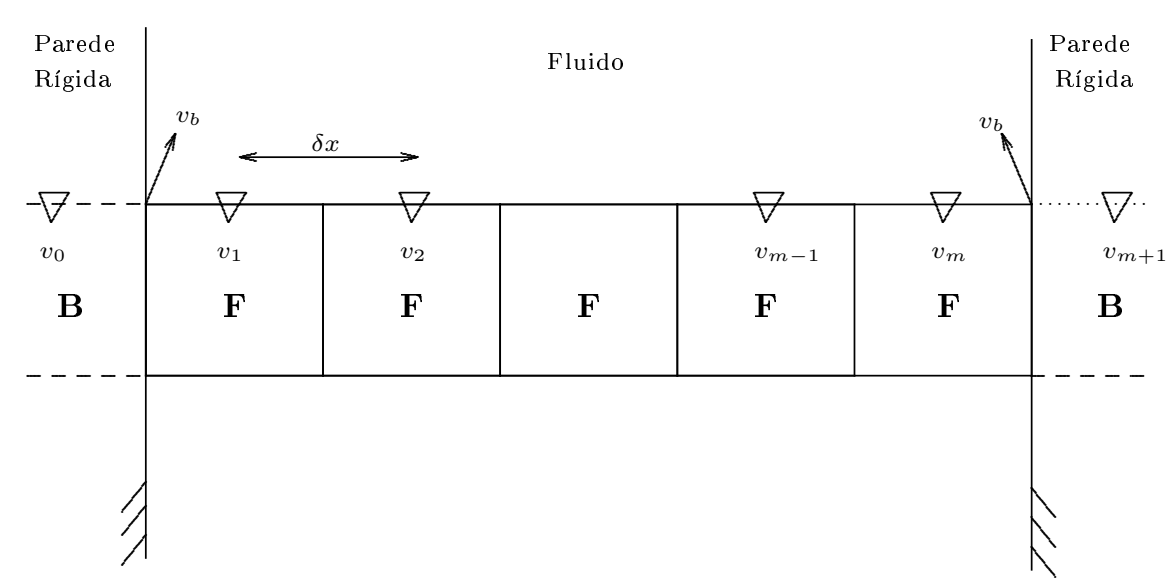

Figura 3.1: Ilustração de uma malha deslocada e classificação de célula em um canal vertical. Neste caso, a velocidade na parede $v_{b}=0$, enquanto que as velocidades internas $v_{1}, v_{2}, \ldots, v_{m-1}, v_{m}$ são calculadas pela equação de quantidade de movimento (2.22).

Desta forma, o problema modelo escolhido é a equação do calor unidimensional

$$
u_{t}=u_{x x}, \quad x \in[0,1] \quad \text { e } \quad t>0
$$


com condição inicial dada por

$$
u(x, 0)=f(x)
$$

e condições de contorno do tipo Dirichlet

$$
u(0, t)=g(t) \quad \text { e } \quad u(1, t)=h(t)
$$

Como o interesse desse estudo é a estabilidade de métodos numéricos para equações de Navier-Stokes com condições de contorno em paredes rígidas, onde a condição sem escorregamento é imposta, assume-se que em (3.3) as funções $g(t)$ e $h(t)$ são constantes. Com o objetivo de estudar o efeito da malha deslocada na discretização das equações de Navier-Stokes, aproxima-se a equação do calor e suas condições de contorno no intervalo $[0,1]$ pelos seguintes passos:

1. Discretize o intervalo $[0,1]$ por um conjunto de pontos igualmente espaçados $x_{i}=(i-$ $\left.\frac{1}{2}\right) \delta x, i=0,1, \ldots, m+1$ onde $\delta x=\frac{1}{m}$;

2. Nos pontos internos $x_{1}, x_{2}, \ldots, x_{m}$, aproxime a equação do calor (3.1) por um esquema numérico;

3. Como na discretização os pontos $x_{0}$ e $x_{m+1}$ não coincidem com os pontos inicial e final do intervalo [0,1], uma interpolação deve ser usada para eliminar os valores desconhecidos de $u_{0}$ e $u_{m+1}$ das equações obtidas no passo 2 . Neste capítulo, utiliza-se a notação $u_{i}^{(n)}$ para denotar uma aproximação para $u\left(x_{i}, t_{n}\right)$.

A figura 3.2 ilustra a malha deslocada usada na solução da equação (3.1), e representa uma faixa horizontal para o canal vertical descrito anteriormente (figura 3.1).

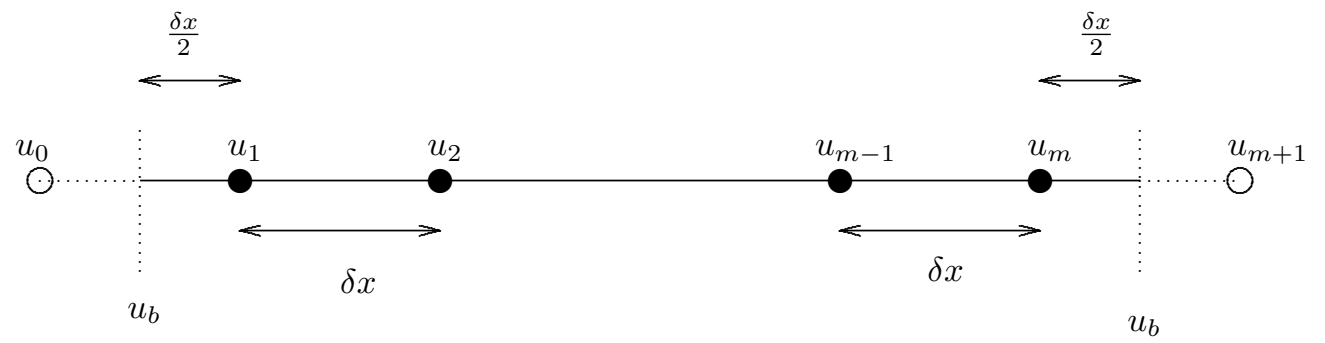

Figura 3.2: Malha deslocada para a solução da equação (3.1) com condições de contorno (3.3), em que $u(0, t)=u(1, t)=u_{b}=0$.

Uma interpolação linear geralmente é usada para aproximar os valores nos contornos no passo 3 acima. O polinômio interpolador de grau 1 nos pontos $\left(x_{0}, u_{0}^{l}\right)$ e $\left(x_{1}, u_{1}^{l}\right)$, onde $l$ é um 
nível de tempo genérico, é dado por

$$
P_{1}(x)=\frac{1}{\delta x}\left(\left(x-x_{0}\right) u_{1}^{l}-\left(x-x_{1}\right) u_{0}^{l}\right) .
$$

Usando a condição de contorno $u(0, t)=0$, tem-se que

$$
P_{1}(0)=0=\frac{1}{\delta x}\left(\frac{\delta x}{2}\left(u_{1}^{l}+u_{0}^{l}\right)\right)=\frac{1}{2}\left(u_{0}^{l}+u_{1}^{l}\right)
$$

Analogamente, a interpolação no ponto final $x=1$ determina a equação

$$
\frac{1}{2}\left(u_{m+1}^{l}+u_{m}^{l}\right)=0
$$

Desta forma, as equações para os valores desconhecidos $u_{0}^{l}$ e $u_{m+1}^{l}$ tornam-se

$$
u_{0}^{l}=-u_{1}^{l} \quad \text { e } \quad u_{m+1}^{l}=-u_{m}^{l}
$$

Neste estudo, aplica-se os casos onde $l$ assume os valores de $n$ ou $n+1$.

A discretização da equação (3.1) pelo do método de diferenças finitas, utilizando um esquema explícito, nos pontos internos $x_{1}, x_{2}, \ldots x_{m}$ resulta em

$$
u_{i}^{(n+1)}=\sigma u_{i+1}^{(n)}+(1-2 \sigma) u_{i}^{(n)}+\sigma u_{i-1}^{(n)},
$$

pelo método de Euler implícito resulta em

$$
-\sigma u_{i-1}^{(n+1)}+(1+2 \sigma) u_{i}^{(n+1)}-\sigma u_{i+1}^{(n+1)}=u_{i}^{(n)},
$$

e pelo método de Crank-Nicolson resulta em

$$
-\frac{\sigma}{2} u_{i-1}^{(n+1)}+(1+\sigma) u_{i}^{(n+1)}-\frac{\sigma}{2} u_{i+1}^{(n+1)}=\frac{\sigma}{2} u_{i-1}^{(n)}+(1-\sigma) u_{i}^{(n)}+\frac{\sigma}{2} u_{i+1}^{(n)},
$$

onde $\sigma=\frac{\delta t}{(\delta x)^{2}}$. Observe que quaisquer dos métodos numéricos acima com quaisquer condições de contorno podem ser escrito na forma matricial como

$$
\mathrm{A} \mathbf{u}^{(n+1)}=\mathrm{Bu}^{(n)}+\mathbf{c},
$$

na qual A e B são matrizes $m \times m, \mathbf{u}=\left(u_{1}, u_{2}, \ldots, u_{m}\right)^{T}$ e $\mathbf{c}=\left(c_{1}, c_{2}, \ldots, c_{m}\right)^{T}$ são vetores $m \times 1$, todos estes definidos de acordo com a escolha particular do método e da condição de contorno. 
A estabilidade numérica do método será determinada pelos autovalores $\lambda$ da matriz de iteração $M=A^{-1} B$. Se todos os autovalores dessa matriz tiverem módulo menor que um, o método será estável. Se no mínimo um autovalor tiver módulo maior ou igual a um, o método será instável. Note que os autovalores dependerão do parâmetro $\sigma$. Quando todos autovalores da matriz de iteração tiverem módulo menor que um para qualquer $\sigma>0$, o método é incondicionalmente estável, se isto for verdade apenas para $\sigma$ em um intervalo finito, o método é condicionalmente estável. Na próxima seção a estabilidade de 6 escolhas diferentes de métodos e condições de contorno serão analisadas. Os diferentes métodos e condições de contorno são definidos como:

1. Método explícito com condições de contorno explícitas - condicionalmente estável para $\sigma \in\left[0, \frac{1}{2}\right)$

2. Método de Euler implícito com condições de contorno implícitas - incondicionalmente estável;

3. Método de Euler implícito com condições de contorno explícitas - incondicionalmente estável;

4. Método de Crank-Nicolson com condições de contorno implícitas do tipo 1 - incondicionalmente estável;

5. Método de Crank-Nicolson com condições de contorno implícitas do tipo 2 - incondicionalmente estável;

6. Método de Crank-Nicolson com condições de contorno explícitas - condicionalmente estável para $\sigma \in[0,2)$.

Para o método explícito, as condições de contorno explícitas em (3.7) são

$$
u_{0}^{(n)}=-u_{1}^{(n)} \quad \text { e } \quad u_{m+1}^{(n)}=-u_{m}^{(n)}
$$

Para o método de Euler implícito tem-se

$$
u_{0}^{(n+1)}=-u_{1}^{(n+1)} \quad \text { e } \quad u_{m+1}^{(n+1)}=-u_{m}^{(n+1)}
$$

para condições de contorno implícitas, e

$$
u_{0}^{(n+1)}=-u_{1}^{(n)} \quad \text { e } \quad u_{m+1}^{(n+1)}=-u_{m}^{(n)},
$$


no caso das condições de contorno explícitas. Finalmente, para o método de Crank-Nicolson tem-se

$$
u_{0}^{(n+1)}=-u_{1}^{(n+1)}, \quad u_{m+1}^{(n+1)}=-u_{m}^{(n+1)}, \quad u_{0}^{(n)}=-u_{1}^{(n)} \quad \text { e } \quad u_{m+1}^{(n)}=-u_{m}^{(n)},
$$

no caso de condições de contorno implícitas, e

$$
u_{0}^{(n+1)}=-u_{1}^{(n)}, \quad u_{m+1}^{(n+1)}=-u_{m}^{(n)}, \quad u_{0}^{(n)}=-u_{1}^{(n)} \quad \text { e } \quad u_{m+1}^{(n)}=-u_{m}^{(n)}
$$

para condições de contorno explícitas. Para o método de Crank-Nicolson, outro tipo de condição de contorno implícita será apresentado em detalhes na subseção 3.3.5.

\subsection{Análise de estabilidade}

A análise de estabilidade dos métodos descritos na seção anterior será baseada em teoremas importantes da álgebra linear relativos aos autovalores de uma matriz geral e de matrizes tridiagonais, e um resultado sobre as derivadas de uma função. Para matrizes gerais, resultados importantes para estimar seus autovalores são os teoremas de Gershgorin. Esses teoremas determinam a localização dos autovalores. Para matrizes tridiagonais, Yueh [180] apresenta expressões exatas em alguns casos especiais. Os teoremas de Rayleigh-Ritz e Danskin são apresentados na sequência.

Teorema 1 Considere $\mathrm{A} \in \mathbb{C}^{m \times m}$. Então

$$
\sigma(\mathrm{A}) \subseteq U_{R}=\bigcup_{i=1}^{m} R_{i}, \quad R_{i}=\left\{z \in \mathbb{C}:\left|z-a_{i i}\right| \leq \sum_{j=1, j \neq i}^{m}\left|a_{i j}\right|\right\}
$$

onde $a_{i j}$ são os elementos da matriz $\mathrm{A}$ para $i, j=1, \ldots, m$ e $\sigma(\mathrm{A})$ é o conjunto de autovalores de A chamado espectro de $\mathrm{A}$. Os conjuntos $R_{i}$ são chamados de círculos de Gershgorin.

A demonstração do teorema 1 é bem conhecida e pode ser encontrada em [133]. 
Teorema 2 Seja uma matriz tridiagonal da seguinte forma

$$
\mathrm{P}=\left[\begin{array}{ccccc}
-\alpha+b & c & 0 & 0 & \ldots \\
a & b & c & 0 & \cdots \\
0 & \ddots & \ddots & \ddots & 0 \\
\cdots & 0 & a & b & c \\
\cdots & 0 & 0 & a & -\beta+b
\end{array}\right]_{m \times m}
$$

Os autovalores $\lambda_{i}^{\mathrm{P}}$ de $\mathrm{P}$ são dados por

$$
\lambda_{i}^{\mathrm{P}}= \begin{cases}b+2 \sqrt{a c} \cos \left(\frac{i \pi}{m+1}\right), & i=1, \ldots, m, \quad \text { se } \quad \alpha=\beta=0, \\ b+2 \sqrt{a c} \cos \left(\frac{i \pi}{m}\right), & i=1, \ldots, m, \quad \text { se } \quad \alpha=\beta=\sqrt{a c} \neq 0 \\ b+2 \sqrt{a c} \cos \left(\frac{(i-1) \pi}{m}\right), & i=1, \ldots, m, \quad \text { se } \quad \alpha=\beta=-\sqrt{a c} \neq 0 .\end{cases}
$$

Detalhes sobre a demonstração deste teorema podem ser encontrados em [180].

Teorema 3 (Rayleigh-Ritz). Seja $\mathrm{A} \in \mathbb{R}^{p \times p}$ uma matriz simétrica, e sejam os autovalores de A ordenados como

$$
\lambda_{\text {min }}^{\mathrm{A}}=\lambda_{1}^{\mathrm{A}} \leq \lambda_{2}^{\mathrm{A}} \leq \ldots \lambda_{p-1}^{\mathrm{A}} \leq \lambda_{p}^{\mathrm{A}}=\lambda_{\text {max }}^{\mathrm{A}}
$$

\section{Então}

$$
\begin{gathered}
\lambda_{1}^{\mathrm{A}} \boldsymbol{v}^{T} \boldsymbol{v} \leq \boldsymbol{v}^{T} \mathrm{~A} \boldsymbol{v} \leq \lambda_{p}^{\mathrm{A}} \boldsymbol{v}^{T} \boldsymbol{v} \quad \text { para todo } \quad \boldsymbol{v} \in \mathbb{R}^{p} \\
\lambda_{\text {min }}^{\mathrm{A}}=\lambda_{1}^{\mathrm{A}}=\min _{\boldsymbol{v} \neq 0} \frac{\boldsymbol{v}^{T} \mathrm{~A} \boldsymbol{v}}{\boldsymbol{v}^{T} \boldsymbol{v}}, \\
\lambda_{\text {max }}^{\mathrm{A}}=\lambda_{p}^{\mathrm{A}}=\max _{\boldsymbol{v} \neq 0} \frac{\boldsymbol{v}^{T} \mathrm{~A} \boldsymbol{v}}{\boldsymbol{v}^{T} \boldsymbol{v}} .
\end{gathered}
$$

A demonstração deste teorema pode ser encontrada em [73].

Teorema 4 (Danskin). Suponha que $f: X \times Y \rightarrow \mathbb{R}$ é uma função contínua, onde $X \subset \mathbb{R}^{n}$ é um conjunto aberto, $Y$ é um conjunto compacto de um espaço topológico $F$, e $\nabla_{x} f(x, y)$ existe e é contínuo. Então a função

$$
\phi(x)=\min _{y \in Y} f(x, y)
$$


é contínua e tem derivada direcional em toda direção, dada pela seguinte fórmula

$$
D_{h} \phi(x)=\min _{y \in Y(x)} \nabla_{x} f(x, y)^{T} h
$$

onde $Y(x)=\{y \in Y \mid \phi(x)=f(x, y)\}$ é um conjunto de minimizadores na definição de $\phi(x)$.

Mais detalhes sobre este teorema podem ser encontrados em [32, 64].

Nas próximas demonstrações utiliza-se a definição $M=A^{-1} B$.

\subsubsection{Método explícito com condições de contorno explícitas}

Neste caso, as matrizes em (3.11) são $\mathrm{A}=\mathrm{I}$ e

$$
\mathrm{B}=\left[\begin{array}{ccccc}
1-3 \sigma & \sigma & 0 & 0 & \cdots \\
\sigma & 1-2 \sigma & \sigma & 0 & \cdots \\
0 & \ddots & \ddots & \ddots & 0 \\
\cdots & 0 & \sigma & 1-2 \sigma & \sigma \\
\cdots & 0 & 0 & \sigma & 1-3 \sigma
\end{array}\right]_{m \times m}=\mathrm{I}+\sigma \tilde{\mathrm{B}}
$$

onde

$$
\tilde{\mathrm{B}}=\left[\begin{array}{ccccc}
-3 & 1 & 0 & 0 & \cdots \\
1 & -2 & 1 & 0 & \cdots \\
0 & \ddots & \ddots & \ddots & 0 \\
\cdots & 0 & 1 & -2 & 1 \\
\cdots & 0 & 0 & 1 & -3
\end{array}\right]_{m \times m}
$$

Teorema 5 O módulo de todos os autovalores de $\mathrm{M}$ é menor que 1 se $\sigma \in\left(0, \frac{1}{2}\right)$.

Prova. A matriz $\tilde{\mathrm{B}}$ satisfaz as hipóteses do teorema 2 , com $\alpha=\beta=1, a=c=1$ e $b=-2$. Então, seus autovalores podem ser calculados por (3.19) determinando $\lambda_{i}^{\tilde{\mathrm{B}}}=-2+2 \cos \left(\frac{i \pi}{m}\right)$ para $i=1, \ldots, m$. Portanto, os autovalores de B são

$$
\lambda_{i}^{\mathrm{B}}=1-2 \sigma\left(1-\cos \left(\frac{i \pi}{m}\right)\right)=1-2 \sigma\left(2 \operatorname{sen}^{2}\left(\frac{i \pi}{2 m}\right)\right)=1-4 \sigma \operatorname{sen}^{2}\left(\frac{i \pi}{2 m}\right)
$$


para $i=1, \ldots, m$. Como $\mathrm{A}=\mathrm{I}$, os autovalores de $\mathrm{M}$ são os mesmos que em (3.24). $\mathrm{O}$ autovalor máximo ocorre quando $i=1$, isto é,

$$
\lambda_{\max }^{\mathrm{M}}=\lambda_{1}^{\mathrm{M}}=1-4 \sigma \operatorname{sen}^{2}\left(\frac{\pi}{2 m}\right)
$$

e o autovalor mínimo é

$$
\lambda_{m i n}^{\mathrm{M}}=\lambda_{m}^{\mathrm{M}}=1-4 \sigma \operatorname{sen}^{2}\left(\frac{\pi}{2}\right)=1-4 \sigma .
$$

Neste caso, deve-se mostrar que $\lambda_{\max }^{\mathrm{M}}<1$ e $\lambda_{\min }^{\mathrm{M}}>-1$. Da equação (3.25) é fácil verificar que $\lambda_{\max }^{\mathrm{M}}<1$ para todo $\sigma>0$, e de (3.26) tem-se

$$
\lambda_{\min }^{\mathrm{M}}>-1 \Rightarrow \sigma<\frac{1}{2} .
$$

Este resultado demonstra um fato muito conhecido, que o método explícito é estável para $\sigma \in\left(0, \frac{1}{2}\right)$.

\subsubsection{Método de Euler implícito com condições de contorno implícitas}

Para este caso, utilizando as condições de contorno implícitas (3.13), a matriz B = I, A é dada por

$$
\mathrm{A}=\left[\begin{array}{ccccc}
1+3 \sigma & -\sigma & 0 & 0 & \ldots \\
-\sigma & 1+2 \sigma & -\sigma & 0 & \ldots \\
0 & \ddots & \ddots & \ddots & 0 \\
\cdots & 0 & -\sigma & 1+2 \sigma & -\sigma \\
\cdots & 0 & 0 & -\sigma & 1+3 \sigma
\end{array}\right]_{m \times m}=\mathrm{I}-\sigma \tilde{\mathrm{A}}
$$

onde

$$
\tilde{\mathrm{A}}=\left[\begin{array}{ccccc}
-3 & 1 & 0 & 0 & \cdots \\
1 & -2 & 1 & 0 & \cdots \\
0 & \ddots & \ddots & \ddots & 0 \\
\cdots & 0 & 1 & -2 & 1 \\
\cdots & 0 & 0 & 1 & -3
\end{array}\right]_{m \times m}
$$


Teorema 6 O módulo de todos os autovalores de $\mathrm{M}$ é menor que 1.

Prova. Note que a matriz $\tilde{\mathrm{A}}$ satisfaz as condições do teorema 2, e portanto seus autovalores podem ser calculados de (3.19) como

$$
\lambda_{i}^{\tilde{\mathrm{A}}}=-2+2 \cos \left(\frac{i \pi}{m}\right) \quad \text { para } \quad i=1, \ldots, m .
$$

De (3.28), os autovalores de A são dados por

$$
\begin{aligned}
\lambda_{i}^{\mathrm{A}} & =1-\sigma\left(-2+2 \cos \left(\frac{i \pi}{m}\right)\right)=1+2 \sigma\left(1-\cos \left(\frac{i \pi}{m}\right)\right) \\
& =1+4 \sigma\left(\operatorname{sen}^{2}\left(\frac{i \pi}{2 m}\right)\right), \quad i=1, \ldots, m
\end{aligned}
$$

Logo, da equação (3.31) tem-se que

$$
\left|\lambda_{i}^{\mathrm{A}}\right|>1, \quad \forall \sigma>0 \quad \text { ou } \quad\left|\frac{1}{\lambda_{i}^{\mathrm{A}}}\right|<1, \quad \forall \sigma>0 .
$$

Portanto os autovalores da matriz de iteração $M$ satisfazem

$$
\left|\lambda_{i}^{\mathrm{M}}\right|<1, \quad \forall \sigma>0
$$

e isto prova o teorema.

\subsubsection{Método de Euler implícito com condições de contorno explícitas}

Para o caso do método de Euler implícito com condições de contorno explícitas (ver equações (3.14)), as matrizes A e B de (3.11) tornam-se

$$
\mathrm{A}=\left[\begin{array}{ccccc}
1+2 \sigma & -\sigma & 0 & 0 & \ldots \\
-\sigma & 1+2 \sigma & -\sigma & 0 & \ldots \\
0 & \ddots & \ddots & \ddots & 0 \\
\cdots & 0 & -\sigma & 1+2 \sigma & -\sigma \\
\cdots & 0 & 0 & -\sigma & 1+2 \sigma
\end{array}\right]_{m \times m}=\mathrm{I}+\sigma \tilde{\mathrm{A}}
$$

e

$$
\mathbf{B}=\operatorname{diag}(1-\sigma, 1, \ldots, 1,1-\sigma),
$$


onde

$$
\tilde{\mathrm{A}}=\left[\begin{array}{ccccc}
2 & -1 & 0 & 0 & \ldots \\
-1 & 2 & -1 & 0 & \ldots \\
0 & \ddots & \ddots & \ddots & 0 \\
\cdots & 0 & -1 & 2 & -1 \\
\cdots & 0 & 0 & -1 & 2
\end{array}\right]_{m \times m}
$$

Assim a matriz de iteração é $\mathrm{M}=\mathrm{A}^{-1} \mathrm{~B}=(\mathrm{I}+\sigma \tilde{\mathrm{A}})^{-1} \mathrm{~B}$. Se $\lambda_{i}^{\mathrm{M}}$ são os autovalores de $\mathrm{M}$, então $1 / \lambda_{i}^{\mathrm{M}}$ são os autovalores de $\mathrm{M}^{-1}$.

Agora, pode-se reescrever $\mathrm{M}^{-1}=\mathrm{B}^{-1}(\mathrm{I}+\sigma \tilde{\mathrm{A}})$, ou seja,

$$
\mathrm{M}^{-1}=\left[\begin{array}{ccccc}
\frac{1+2 \sigma}{1-\sigma} & \frac{-\sigma}{1-\sigma} & 0 & 0 & \ldots \\
-\sigma & 1+2 \sigma & -\sigma & 0 & \ldots \\
0 & \ddots & \ddots & \ddots & 0 \\
\cdots & 0 & -\sigma & 1+2 \sigma & -\sigma \\
\cdots & 0 & 0 & \frac{-\sigma}{1-\sigma} & \frac{1+2 \sigma}{1-\sigma}
\end{array}\right]_{m \times m}
$$

Teorema 7 O módulo de todos os autovalores de $\mathrm{M}$ é menor que 1.

Prova. Para provar este teorema, deve-se mostrar que todos os autovalores de $\mathrm{M}^{-1}$ têm módulo maior que 1. Para isto, pode-se utilizar o teorema 1 de Gershgorin para encontrar limitantes para os autovalores. Da forma da matriz $\mathrm{M}^{-1}$ tem-se que $R_{1}=R_{m}$ e $R_{2}=\ldots=$ $R_{m-1}$, com

$$
R_{1}=\left\{z \in \mathbb{C}:\left|z-\frac{1+2 \sigma}{1-\sigma}\right| \leq\left|\frac{-\sigma}{1-\sigma}\right|\right\}
$$

e

$$
R_{2}=\{z \in \mathbb{C}:|z-(1+2 \sigma)| \leq|-\sigma|+|-\sigma|\}
$$

Sabe-se do teorema 1 que todo autovalor de $\mathrm{M}^{-1}$ está em $R_{1}$ ou em $R_{2}$. Considere $\alpha$ o número real mais próximo da origem, definido pela intersecção da fronteira de $R_{i}$ com o eixo real, $r$ e $c$, o raio e o centro do circulo de Gershgorin, respectivamente. Considere primeiro um autovalor $\lambda$ em $R_{2}$ : então $|\lambda-(1+2 \sigma)|<r$.

Como $\sigma>0$, o número real $\alpha$ é dado por $\alpha=1+2 \sigma-2 \sigma=1$. Obviamente, qualquer autovalor em $R_{2}$ tem módulo maior que $\alpha$, e portanto $|\lambda|>1$, como pode ser visto na figura 3.3 .

Seja agora $\lambda \in R_{1}$, deve-se considerar três casos distintos: 


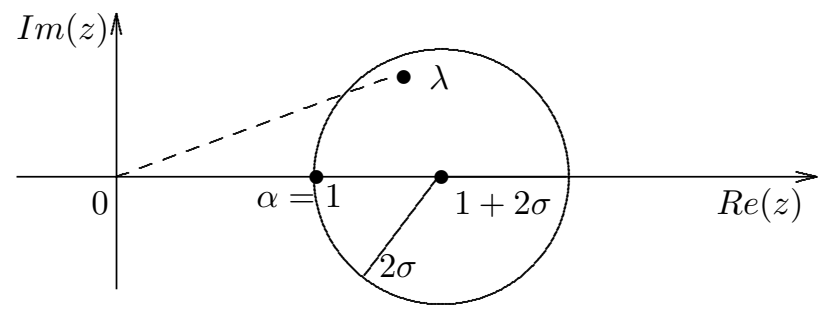

Figura 3.3: Circulo de Gershgorin $R_{2}$.

- Caso 1: $\sigma<1$

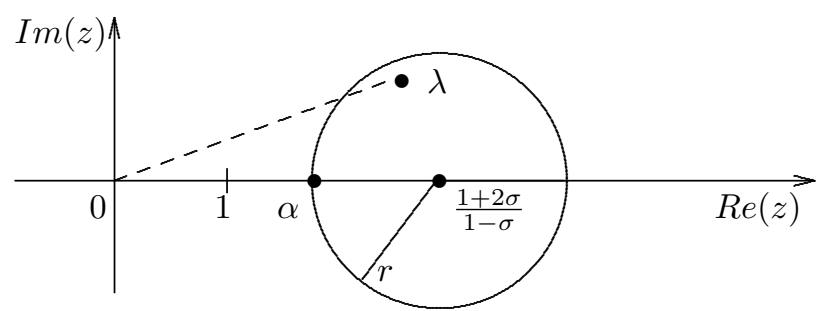

Figura 3.4: Circulo de Gershgorin $R_{1}$ para $\sigma<1$.

Neste caso, o centro do circulo de Gershgorin $R_{1}$ é $c=\frac{1+2 \sigma}{1-\sigma}$, e o raio $r=\left|\frac{-\sigma}{1-\sigma}\right|$. Como $\sigma<1, c>0$ e $r=\frac{\sigma}{1-\sigma}$ tem-se

$$
\alpha=c-r=\frac{1+2 \sigma}{1-\sigma}-\frac{\sigma}{1-\sigma}=\frac{1+\sigma}{1-\sigma}>1
$$

Portanto, qualquer autovalor $\lambda$ de $\mathrm{M}^{-1}$ em $R_{1}$ satisfará $|\lambda|>1$ (ver figura 3.4).

- Caso 2: $\sigma>1$

Como $\sigma>1$, tem-se $c<0$ e $r=\frac{\sigma}{\sigma-1}$. Logo,

$$
\alpha=c+r=\frac{1+2 \sigma}{1-\sigma}+\frac{\sigma}{\sigma-1}=\frac{1+\sigma}{1-\sigma}<-1
$$

Novamente, qualquer autovalor $\lambda$ de $\mathrm{M}^{-1}$ em $R_{1}$ terá seu módulo maior que 1 , como pode ser verificado na figura 3.5. 


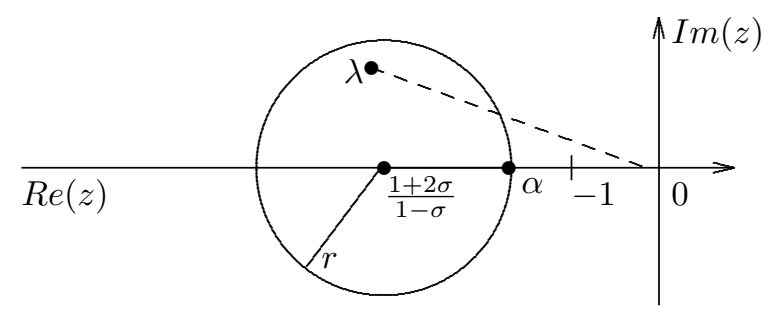

Figura 3.5: Circulo de Gershgorin $R_{1}$ para $\sigma>1$.

- Caso 3: $\sigma=1$

Neste caso, a matriz A, dada por (3.34), torna-se

$$
\mathrm{A}=\left[\begin{array}{ccccc}
3 & -1 & 0 & 0 & \ldots \\
-1 & 3 & -1 & 0 & \ldots \\
0 & \ddots & \ddots & \ddots & 0 \\
\ldots & 0 & -1 & 3 & -1 \\
\ldots & 0 & 0 & -1 & 3
\end{array}\right]_{m \times m}
$$

Note que a matriz (3.42) satisfaz as condições do teorema 2; logo seus autovalores podem ser calculados de (3.19) como

$$
\lambda_{i}^{\mathrm{A}}=3+2 \cos \left(\frac{i \pi}{m+1}\right)>0 \quad \text { para } \quad i=1, \ldots, m .
$$

De (3.43), tem-se que $\lambda_{\text {min }}^{\mathrm{A}}>1 \mathrm{e}\left\|\mathrm{A}^{-1}\right\|_{2}=\frac{1}{\lambda_{\text {min }}^{\mathrm{A}}}$. Agora, substituindo $\sigma=1$ na matriz (3.35), é fácil verificar que $\|\mathrm{B}\|_{2}=1$. Assim, como as matrizes $\mathrm{A}$ e $\mathrm{B}$ são simétricas obtém-se

$$
\left|\lambda_{i}^{\mathrm{M}}\right| \leq\|\mathrm{M}\|_{2}=\left\|\mathrm{A}^{-1} \mathrm{~B}\right\|_{2} \leq\left\|\mathrm{A}^{-1}\right\|_{2}\|\mathrm{~B}\|_{2}=\frac{1}{\lambda_{\min }^{\mathrm{A}}}<1
$$

Portanto, de acordo com os resultados acima, tem-se que todos os autovalores de $\mathrm{M}^{-1}$ têm módulo maior que 1. Desta forma, o teorema 7 está provado. 


\subsubsection{Método de Crank-Nicolson com condições de contorno implíci- tas do tipo 1}

Neste caso, considera-se o método de Crank-Nicolson com condições de contorno implícitas (3.15), para o qual obtém-se as seguintes matrizes

$$
\mathrm{A}=\left[\begin{array}{ccccc}
1+\frac{3}{2} \sigma & -\frac{\sigma}{2} & 0 & 0 & \ldots \\
-\frac{\sigma}{2} & 1+\sigma & -\frac{\sigma}{2} & 0 & \ldots \\
0 & \ddots & \ddots & \ddots & 0 \\
\cdots & 0 & -\frac{\sigma}{2} & 1+\sigma & -\frac{\sigma}{2} \\
\cdots & 0 & 0 & -\frac{\sigma}{2} & 1+\frac{3}{2} \sigma
\end{array}\right]_{m \times m}=\mathrm{I}+\sigma \tilde{\mathrm{A}}
$$

e

$$
\mathrm{B}=\left[\begin{array}{ccccc}
1-\frac{3}{2} \sigma & \frac{\sigma}{2} & 0 & 0 & \ldots \\
\frac{\sigma}{2} & 1-\sigma & \frac{\sigma}{2} & 0 & \cdots \\
0 & \ddots & \ddots & \ddots & 0 \\
\cdots & 0 & \frac{\sigma}{2} & 1-\sigma & \frac{\sigma}{2} \\
\cdots & 0 & 0 & \frac{\sigma}{2} & 1-\frac{3}{2} \sigma
\end{array}\right]_{m \times m}=\mathrm{I}-\sigma \tilde{\mathrm{A}}
$$

onde

$$
\tilde{\mathrm{A}}=\left[\begin{array}{ccccc}
\frac{3}{2} & -\frac{1}{2} & 0 & 0 & \ldots \\
-\frac{1}{2} & 1 & -\frac{1}{2} & 0 & \cdots \\
0 & \ddots & \ddots & \ddots & 0 \\
\cdots & 0 & -\frac{1}{2} & 1 & -\frac{1}{2} \\
\cdots & 0 & 0 & -\frac{1}{2} & \frac{3}{2}
\end{array}\right]_{m \times m}
$$

Teorema 8 O módulo de todos os autovalores de $\mathrm{M}$ é menor que 1.

Prova. A matriz $\tilde{A}$ satisfaz as condições do teorema 2; logo seus autovalores podem ser calculados de (3.19) da seguinte forma

$$
\lambda_{i}^{\tilde{\mathrm{A}}}=1+\cos \left(\frac{(i-1) \pi}{m}\right), \quad i=1, \ldots, m .
$$

Assim, os autovalores de A e B são

$$
\lambda_{i}^{\mathrm{A}}=1+\sigma\left(1+\cos \left(\frac{(i-1) \pi}{m}\right)\right), \quad i=1, \ldots, m
$$




$$
\lambda_{i}^{\mathrm{B}}=1-\sigma\left(1+\cos \left(\frac{(i-1) \pi}{m}\right)\right), \quad i=1, \ldots, m,
$$

respectivamente. Note que, as matrizes A e B têm os mesmos autovetores; e portanto os autovalores da matriz de iteração $M$ satisfazem:

$$
\left|\lambda_{i}^{\mathrm{M}}\right|=\left|\frac{\lambda_{i}^{\mathrm{B}}}{\lambda_{i}^{\mathrm{A}}}\right|=\left|\frac{1-\sigma\left(1+\cos \left(\frac{(i-1) \pi}{m}\right)\right)}{1+\sigma\left(1+\cos \left(\frac{(i-1) \pi}{m}\right)\right)}\right|<1, \quad \forall \sigma>0,
$$

e isto prova o teorema.

\subsubsection{Método de Crank-Nicolson com condições de contorno implíci- tas do tipo 2}

Este caso é absolutamente artificial, e é apresentado apenas para mostrar como as condições de contorno podem afetar a estabilidade de um esquema numérico. O método de Crank-Nicolson é aplicado com as condições de contorno implícitas

$$
u_{0}^{(n+1)}=-u_{1}^{(n+1)}, \quad u_{m+1}^{(n+1)}=-u_{m}^{(n+1)}, \quad u_{0}^{(n)}=-u_{1}^{(n+1)} \quad \text { e } \quad u_{m+1}^{(n)}=-u_{m}^{(n+1)}
$$

Neste caso tem-se as seguintes matrizes

$$
\mathrm{A}=\left[\begin{array}{ccccc}
1+2 \sigma & -\frac{\sigma}{2} & 0 & 0 & \ldots \\
-\frac{\sigma}{2} & 1+\sigma & -\frac{\sigma}{2} & 0 & \ldots \\
0 & \ddots & \ddots & \ddots & 0 \\
\cdots & 0 & -\frac{\sigma}{2} & 1+\sigma & -\frac{\sigma}{2} \\
\cdots & 0 & 0 & -\frac{\sigma}{2} & 1+2 \sigma
\end{array}\right]_{m \times m}=\mathrm{I}+\sigma(\tilde{\mathrm{A}}+\mathrm{E})
$$

e

$$
\mathrm{B}=\left[\begin{array}{ccccc}
1-\sigma & \frac{\sigma}{2} & 0 & 0 & \cdots \\
\frac{\sigma}{2} & 1-\sigma & \frac{\sigma}{2} & 0 & \cdots \\
0 & \ddots & \ddots & \ddots & 0 \\
\cdots & 0 & \frac{\sigma}{2} & 1-\sigma & \frac{\sigma}{2} \\
\cdots & 0 & 0 & \frac{\sigma}{2} & 1-\sigma
\end{array}\right]_{m \times m}=\mathrm{I}-\sigma \tilde{\mathrm{A}}
$$


onde

$$
\tilde{\mathrm{A}}=\left[\begin{array}{ccccc}
1 & -\frac{1}{2} & 0 & 0 & \ldots \\
-\frac{1}{2} & 1 & -\frac{1}{2} & 0 & \ldots \\
0 & \ddots & \ddots & \ddots & 0 \\
\cdots & 0 & -\frac{1}{2} & 1 & -\frac{1}{2} \\
\ldots & 0 & 0 & -\frac{1}{2} & 1
\end{array}\right]_{m \times m}
$$

e

$$
\mathrm{E}=\operatorname{diag}(1,0, \ldots, 0,1)
$$

Teorema 9 O módulo de todos os autovalores de $\mathrm{M}$ é menor que 1.

Prova. De acordo com (3.53) e (3.54), a matriz de iteração é dada por

$$
\begin{aligned}
\mathrm{M}=\mathrm{A}^{-1} \mathrm{~B} & =(\mathrm{I}+\sigma(\tilde{\mathrm{A}}+\mathrm{E}))^{-1}(\mathrm{I}-\sigma \tilde{\mathrm{A}}) \\
& =(\mathrm{I}+\sigma(\tilde{\mathrm{A}}+\mathrm{E}))^{-1}(\mathrm{I}+\sigma(\tilde{\mathrm{A}}+\mathrm{E})-\sigma(2 \tilde{\mathrm{A}}+\mathrm{E})) \\
& =\mathrm{I}-(\mathrm{I}+\sigma(\tilde{\mathrm{A}}+\mathrm{E}))^{-1} \sigma(2 \tilde{\mathrm{A}}+\mathrm{E}) \\
& =\mathrm{I}-((1 / \sigma) \mathrm{I}+\tilde{\mathrm{A}}+\mathrm{E})^{-1}(2 \tilde{\mathrm{A}}+\mathrm{E})=\mathrm{I}-\tilde{\mathrm{M}},
\end{aligned}
$$

onde $\tilde{\mathrm{M}}=((1 / \sigma) \mathbf{I}+\tilde{\mathrm{A}}+\mathrm{E})^{-1}(2 \tilde{\mathrm{A}}+\mathrm{E})$. Se $\lambda_{i}^{\tilde{\mathrm{M}}}$ são os autovalores de $\tilde{\mathrm{M}}$, então $1-\lambda_{i}^{\tilde{\mathrm{M}}}$ são os autovalores de $\mathrm{M}$.

Suponha que $\tilde{M} \mathbf{v}=\lambda \mathbf{v}$ com $\|\mathbf{v}\|_{2}=1$. Logo tem-se

$$
\begin{aligned}
& \left(\frac{1}{\sigma} \mathrm{I}+\tilde{\mathrm{A}}+\mathrm{E}\right)^{-1}(2 \tilde{\mathrm{A}}+\mathrm{E}) \mathbf{v}=\lambda_{i}^{\tilde{\mathrm{M}}} \mathbf{v} \\
& \Rightarrow(2 \tilde{\mathrm{A}}+\mathrm{E}) \mathbf{v}=\lambda_{i}^{\tilde{\mathrm{M}}}\left(\frac{1}{\sigma} \mathrm{I}+\tilde{\mathrm{A}}+\mathrm{E}\right) \mathbf{v} \\
& \Rightarrow 2 \mathbf{v}^{T} \tilde{\mathrm{A}} \mathbf{v}+\mathbf{v}^{T} \mathrm{E} \mathbf{v}=\lambda_{i}^{\tilde{\mathrm{M}}}\left(\frac{1}{\sigma}+\mathbf{v}^{T} \tilde{\mathrm{A}} \mathbf{v}+\mathbf{v}^{T} \mathrm{E} \mathbf{v}\right) \\
& \Rightarrow 2 \mathbf{v}^{T} \tilde{\mathrm{A}} \mathbf{v}+v_{1}^{2}+v_{m}^{2}=\lambda_{i}^{\tilde{\mathrm{M}}}\left((1 / \sigma)+\mathbf{v}^{T} \tilde{\mathrm{A}} \mathbf{v}+v_{1}^{2}+v_{m}^{2}\right)
\end{aligned}
$$

Desta forma, os autovalores $\lambda_{i}^{\tilde{M}}$ podem ser escritos como

$$
\lambda_{i}^{\tilde{\mathrm{M}}}=\frac{2 \mathbf{v}^{T} \tilde{\mathrm{A}} \mathbf{v}+v_{1}^{2}+v_{m}^{2}}{(1 / \sigma)+\mathbf{v}^{T} \tilde{\mathrm{A}} \mathbf{v}+v_{1}^{2}+v_{m}^{2}} .
$$


Como a matriz $\tilde{\mathrm{A}}$ é simétrica e definida positiva, de acordo com $\lambda_{i}^{\tilde{\mathrm{A}}}>0$ de (3.19), então $\lambda_{i}^{\tilde{\mathrm{M}}}>0$. Utilizando (3.59), tem-se que

$$
0<\lambda_{i}^{\tilde{\mathrm{M}}}=\frac{2 \mathbf{v}^{T} \tilde{\mathrm{A}} \mathbf{v}+v_{1}^{2}+v_{m}^{2}}{(1 / \sigma)+\mathbf{v}^{T} \tilde{\mathrm{A}} \mathbf{v}+v_{1}^{2}+v_{m}^{2}}<\frac{2 \mathbf{v}^{T} \tilde{\mathrm{A}} \mathbf{v}+2 v_{1}^{2}+2 v_{m}^{2}+\frac{2}{\sigma}}{(1 / \sigma)+\mathbf{v}^{T} \tilde{\mathrm{A}} \mathbf{v}+v_{1}^{2}+v_{m}^{2}}=2
$$

Portanto, como $\lambda_{i}^{\mathrm{M}}=1-\lambda_{i}^{\tilde{\mathrm{M}}}$ e $0<\lambda_{i}^{\tilde{\mathrm{M}}}<2$, os autovalores da matriz de iteração $\mathrm{M}$ satisfazem

$$
-1<\lambda_{i}^{\mathrm{M}}<1, \quad \forall \sigma>0
$$

e isto prova o teorema.

\subsubsection{Método de Crank-Nicolson com condições de contorno explíci- $\operatorname{tas}$}

Para este caso, as condições de contorno explícitas são dadas por (3.16), e as matrizes A e B são

$$
\mathrm{A}=\left[\begin{array}{ccccc}
1+\sigma & -\frac{\sigma}{2} & 0 & 0 & \ldots \\
-\frac{\sigma}{2} & 1+\sigma & -\frac{\sigma}{2} & 0 & \cdots \\
0 & \ddots & \ddots & \ddots & 0 \\
\cdots & 0 & -\frac{\sigma}{2} & 1+\sigma & -\frac{\sigma}{2} \\
\cdots & 0 & 0 & -\frac{\sigma}{2} & 1+\sigma
\end{array}\right]_{m \times m}=\mathrm{I}+\sigma \tilde{\mathrm{A}}
$$

e

$$
\mathrm{B}=\left[\begin{array}{ccccc}
1-2 \sigma & \frac{\sigma}{2} & 0 & 0 & \ldots \\
\frac{\sigma}{2} & 1-\sigma & \frac{\sigma}{2} & 0 & \ldots \\
0 & \ddots & \ddots & \ddots & 0 \\
\cdots & 0 & \frac{\sigma}{2} & 1-\sigma & \frac{\sigma}{2} \\
\cdots & 0 & 0 & \frac{\sigma}{2} & 1-2 \sigma
\end{array}\right]_{m \times m}=\mathrm{I}+\sigma \tilde{\mathrm{B}}
$$

onde

$$
\tilde{\mathrm{A}}=\left[\begin{array}{ccccc}
1 & -\frac{1}{2} & 0 & 0 & \ldots \\
-\frac{1}{2} & 1 & -\frac{1}{2} & 0 & \ldots \\
0 & \ddots & \ddots & \ddots & 0 \\
\ldots & 0 & -\frac{1}{2} & 1 & -\frac{1}{2} \\
\cdots & 0 & 0 & -\frac{1}{2} & 1
\end{array}\right]_{m \times m}
$$


$\mathrm{e}$

$$
\tilde{\mathrm{B}}=\left[\begin{array}{ccccc}
-2 & \frac{1}{2} & 0 & 0 & \cdots \\
\frac{1}{2} & -1 & \frac{1}{2} & 0 & \cdots \\
0 & \ddots & \ddots & \ddots & 0 \\
\cdots & 0 & \frac{1}{2} & -1 & \frac{1}{2} \\
\cdots & 0 & 0 & \frac{1}{2} & -2
\end{array}\right]_{m \times m}
$$

Teorema 10 Os autovalores da matriz $\mathrm{M}$ satisfazem:

1. $\left|\lambda_{i}^{\mathrm{M}}\right|<1, i=1, \ldots, m$ se $\sigma \in(0,2)$;

2. $\left|\lambda_{i}^{\mathrm{M}}\right| \geq 1$, para algum $i$ se $\sigma \in[2, \infty)$.

Prova. Considere $\lambda$ um autovalor de $\mathrm{M}$ com autovetor $\mathbf{v} \neq 0$, então

$$
\mathbf{M} \mathbf{v}=\lambda \mathbf{v} \quad \text { ou } \quad \mathrm{A}^{-1} \mathrm{~B} \mathbf{v}=\lambda \mathbf{v} \quad \text { ou } \quad \mathrm{B} \mathbf{v}=\lambda \mathrm{A} \mathbf{v} .
$$

Para $\sigma=2$, é fácil verificar que $\mathbf{B e}_{1}=-\mathbf{A e}_{1}$, onde $\mathbf{e}_{1}$ é o vetor canônico $(1,0, \ldots, 0)^{T}$, assim $-\mathbf{e}_{1}=\mathrm{A}^{-1} \mathrm{Be}_{1}=\mathrm{Me}_{1}$. Logo, $\lambda=-1$ é um autovalor de $\mathrm{M}$. De forma semelhante, pode-se mostrar que $\mathrm{Me}_{m}=-\mathbf{e}_{m}$.

Agora, deve-se mostrar que $\lambda_{\min }^{\mathrm{M}}=-1$ para $\sigma=2$. Suponha que exista um autovalor $\lambda<-1$, com

$$
\mathrm{A}^{-1} \mathrm{~B} \mathbf{v}=\lambda \mathbf{v} \quad \text { e } \quad\|\mathbf{v}\|_{2}=1
$$

As matrizes A e B em (3.67) para $\sigma=2$, respectivamente são dadas por

$$
\mathrm{A}=\left[\begin{array}{ccccc}
3 & -1 & 0 & 0 & \ldots \\
-1 & 3 & -1 & 0 & \ldots \\
0 & \ddots & \ddots & \ddots & 0 \\
\ldots & 0 & -1 & 3 & -1 \\
\ldots & 0 & 0 & -1 & 3
\end{array}\right]_{m \times m} \quad \text { e } \mathrm{B}=\left[\begin{array}{ccccc}
-3 & 1 & 0 & 0 & \ldots \\
1 & -1 & 1 & 0 & \ldots \\
0 & \ddots & \ddots & \ddots & 0 \\
\ldots & 0 & 1 & -1 & 1 \\
\ldots & 0 & 0 & 1 & -3
\end{array}\right]_{m \times m}
$$

Da equação (3.67) tem-se

$$
\mathbf{v}^{T} \mathrm{~B} \mathbf{v}=\lambda \mathbf{v}^{T} \mathrm{~A} \mathbf{v}
$$

Note que de (3.68) obtém-se

$$
B=-I+E \quad \text { e } \quad A=I+F,
$$


onde

$$
\mathrm{E}=\left[\begin{array}{ccccc}
-2 & 1 & 0 & 0 & \ldots \\
1 & 0 & 1 & 0 & \ldots \\
0 & \ddots & \ddots & \ddots & 0 \\
\cdots & 0 & 1 & 0 & 1 \\
\cdots & 0 & 0 & 1 & -2
\end{array}\right]_{m \times m} \quad \mathrm{e} \quad \mathrm{F}=\left[\begin{array}{ccccc}
2 & -1 & 0 & 0 & \ldots \\
-1 & 2 & -1 & 0 & \ldots \\
0 & \ddots & \ddots & \ddots & 0 \\
\cdots & 0 & -1 & 2 & -1 \\
\cdots & 0 & 0 & -1 & 2
\end{array}\right]_{m \times m}
$$

Logo, de (3.69) e (3.70) tem-se

$$
-1+\mathbf{v}^{T} \mathrm{E} \mathbf{v}=\lambda+\lambda \mathbf{v}^{T} \mathrm{~F} \mathbf{v}
$$

A matriz $\mathrm{F}$ satisfaz as condições do teorema 2 , com $\alpha=\beta=0, a=c=1$ e $b=2$. Assim, seus autovalores podem ser calculados de (3.19) resultando $\lambda_{i}^{F}=2+2 \cos \left(\frac{i \pi}{m+1}\right)$ para $i=1, \ldots, m$, isto é, $\lambda_{i}^{\mathrm{F}}>0$. Logo, como $\mathrm{F}$ é simétrica e definida positiva e $\lambda<-1$, pode-se escrever

$$
-1+\mathbf{v}^{T} \mathrm{Ev}<-1-\mathbf{v}^{T} \mathrm{~F} \mathbf{v} \Rightarrow \mathbf{v}^{T}(\mathrm{E}+\mathrm{F}) \mathbf{v}<0
$$

Note que

$$
\mathrm{E}+\mathrm{F}=\left[\begin{array}{ccccc}
0 & 0 & 0 & 0 & \cdots \\
0 & 2 & 0 & 0 & \cdots \\
0 & \ddots & \ddots & \ddots & 0 \\
\cdots & 0 & 0 & 2 & 0 \\
\cdots & 0 & 0 & 0 & 0
\end{array}\right]_{m \times m}
$$

Desta forma $\mathbf{v}^{T}(\mathrm{E}+\mathrm{F}) \mathbf{v}=2 \sum_{i=2}^{m-1} v_{i}^{2}$ e não pode ser negativo, assim, não pode existir um autovalor $\lambda<-1$. Portanto, para $\sigma=2, \lambda_{\min }^{\mathrm{M}}=-1$.

Agora, deve-se mostrar que $\lambda_{\min }^{\mathrm{M}}$ e $\lambda_{\max }^{\mathrm{M}}$ são funções monotonicamente decrescentes de $\sigma$.

Primeiro, note que a matriz $M$ é semelhante a uma matriz simétrica $\tilde{M}$. De (3.19) tem-se $\lambda_{i}^{\mathrm{A}}>0$ e consequentemente, a matriz $\mathrm{A}$ é definida positiva, logo existe $\mathrm{A}^{\frac{1}{2}}$ tal que $\mathrm{A}^{\frac{1}{2}} \mathrm{~A}^{\frac{1}{2}}=\mathrm{A}$. Assim

$$
\mathrm{A}^{\frac{1}{2}} \mathrm{MA}^{-\frac{1}{2}}=\mathrm{A}^{-\frac{1}{2}} \mathrm{BA}^{-\frac{1}{2}}=\tilde{\mathrm{M}}
$$


o qual é simétrica, e consequentemente tem apenas autovalores reais. Do teorema 3, tem-se

$$
\lambda_{\min }^{\tilde{\mathrm{M}}}(\sigma)=\min _{\mathbf{v} \neq 0} \frac{\mathbf{v}^{T} \tilde{\mathrm{M}} \mathbf{v}}{\mathbf{v}^{T} \mathbf{v}}=\min _{\mathbf{w} \neq 0} \frac{\mathbf{w}^{T}(\mathbf{I}+\sigma \tilde{\mathrm{B}}) \mathbf{w}}{\mathbf{w}^{T}(\mathbf{I}+\sigma \tilde{\mathrm{A}}) \mathbf{w}},
$$

usando a transformação linear definida positiva $\mathbf{w}=\mathrm{A}^{-\frac{1}{2}} \mathbf{v}$ de $\mathbb{R}^{n}$ para $\mathbb{R}^{n}$.

Agora, deve-se mostrar que $\lambda_{\min }^{\mathrm{M}}(\sigma)$ é uma função monotonicamente decrescente de $\sigma$, para todo $\sigma \geq 0$.

A expressão (3.76) pode ser reescrita como

$$
\lambda_{\min }^{\tilde{\mathrm{M}}}(\sigma)=\min _{\|\mathbf{w}\|=1} \frac{\mathbf{w}^{T}(\mathbf{I}+\sigma \tilde{\mathrm{B}}) \mathbf{w}}{\mathbf{w}^{T}(\mathbf{I}+\sigma \tilde{\mathrm{A}}) \mathbf{w}}
$$

Define-se o conjunto compacto $C=\left\{\mathbf{w} \in \mathbb{R}^{n} \mid\|\mathbf{w}\|=1\right\}$ e a função

$$
\mathbf{w} \in C \mapsto g(\sigma, \mathbf{w})=\frac{\mathbf{w}^{T}(\mathbf{I}+\sigma \tilde{\mathrm{B}}) \mathbf{w}}{\mathbf{w}^{T}(\mathbf{I}+\sigma \tilde{\mathrm{A}}) \mathbf{w}}, \sigma>0 .
$$

Esta função é contínua e continuamente diferenciável em relação a $\sigma$, satisfazendo as hipóteses do teorema de Danskin (ver teorema 4). Então, pode-se calcular a derivada direcional a direita a partir de

$$
\frac{d \lambda_{\min }^{\tilde{\mathrm{M}}}(\sigma)}{d \sigma^{+}}=\min _{\mathbf{w} \in W(\sigma)} \frac{d}{d \sigma^{+}} g(\sigma, \mathbf{w})
$$

onde $W(\sigma)=\left\{\mathbf{w} \in C \mid \lambda_{\text {min }}^{\tilde{\mathrm{M}}}(\sigma)=g(\sigma, \mathbf{w})\right\}$ é um conjunto compacto.

Para $\sigma>0$ e w $\in C$ pode-se calcular a derivada como

$$
\frac{d}{d \sigma^{+}} g(\sigma, \mathbf{w})=\frac{\mathbf{w}^{T}(\tilde{\mathrm{B}}-\tilde{\mathrm{A}}) \mathbf{w} \mathbf{w}^{T} \mathbf{w}}{\left[\mathbf{w}^{T}(\mathrm{I}+\sigma \tilde{\mathrm{A}}) \mathbf{w}\right]^{2}}=\frac{\mathbf{w}^{T}(\tilde{\mathrm{B}}-\tilde{\mathrm{A}}) \mathbf{w}}{\left[\mathbf{w}^{T}(\mathrm{I}+\sigma \tilde{\mathrm{A}}) \mathbf{w}\right]^{2}} .
$$

A matriz $(\tilde{B}-\tilde{A})$ é dada por

$$
\tilde{\mathrm{B}}-\tilde{\mathrm{A}}=\left[\begin{array}{ccccc}
-3 & 1 & 0 & 0 & \ldots \\
1 & -2 & 1 & 0 & \ldots \\
0 & \ddots & \ddots & \ddots & 0 \\
\ldots & 0 & 1 & -2 & 1 \\
\ldots & 0 & 0 & 1 & -3
\end{array}\right]_{m \times m}
$$


e de acordo com o teorema 2, pode-se calcular seus autovalores como

$$
\lambda_{i}^{\tilde{\mathrm{B}}-\tilde{\mathrm{A}}}=-2+2 \cos \left(\frac{i \pi}{m}\right) \quad \text { para } \quad i=1, \ldots, m
$$

e então $\mathbf{w}^{T}(\tilde{\mathrm{B}}-\tilde{\mathrm{A}}) \mathbf{w}<0$ para todo $\mathbf{w} \in C$. Em particular, para todo $\mathbf{w} \in W(\sigma)$,

$$
\frac{d}{d \sigma^{+}} g(\sigma, \mathbf{w})=\frac{\mathbf{w}^{T}(\tilde{\mathrm{B}}-\tilde{\mathrm{A}}) \mathbf{w}}{\left[\mathbf{w}^{T}(\mathrm{I}+\sigma \tilde{\mathrm{A}}) \mathbf{w}\right]^{2}}<0 .
$$

Portanto está provado que

$$
\frac{d \lambda_{m i n}^{\tilde{\mathrm{M}}}(\sigma)}{d \sigma}=\frac{d \lambda_{m i n}^{\mathrm{M}}(\sigma)}{d \sigma}<0
$$

Para o máximo autovalor, o mesmo argumento pode ser aplicado, simplesmente substituindo o mínimo pelo máximo no teorema 3, e então

$$
\frac{d \lambda_{\max }^{\tilde{\mathrm{M}}}(\sigma)}{d \sigma}=\frac{d \lambda_{\max }^{\mathrm{M}}(\sigma)}{d \sigma}<0
$$

isto é, $\lambda_{\max }^{\mathrm{M}}(\sigma)$ também é uma função monotonicamente decrescente de $\sigma$.

Portanto, para todo $\sigma>0$, tem-se que $\lambda_{m i n}^{\mathrm{M}}(\sigma)$ e $\lambda_{m a x}^{\mathrm{M}}(\sigma)$ são funções monotonicamente decrescentes de $\sigma$.

Agora $\lambda_{i}^{\mathrm{M}}(0)=1$, para $i=1, \ldots, m$, o que significa que $\lambda_{\min }^{\mathrm{M}}(0)=\lambda_{\max }^{\mathrm{M}}(0)=1$. Como $\frac{d \lambda_{\max }^{\mathrm{M}}(\sigma)}{d \sigma}<0, \lambda_{\max }^{\mathrm{M}}(\sigma)$ é sempre menor que 1 para todo $\sigma>0$.

Em resumo, provou-se que: $\lambda_{\text {min }}^{\mathrm{M}}(2)=-1, \lambda_{\text {min }}^{\mathrm{M}}(0)=\lambda_{\max }^{\mathrm{M}}(0)=1, \lambda_{\min }^{\mathrm{M}}(\sigma)$ e $\lambda_{\text {max }}^{\mathrm{M}}(\sigma)$ são funções monotonicamente decrescentes de $\sigma$, logo (ver figura 3.6) tem-se que

$$
\left\{\begin{array}{l}
-1<\lambda_{i}^{\mathrm{M}}(\sigma)<1 \quad \text { para } \quad \sigma \in(0,2), \quad \forall i \\
\lambda_{m i n}^{\mathrm{M}}(\sigma) \leq-1 \quad \text { para } \quad \sigma \geq 2
\end{array}\right.
$$

Portanto, de (3.81) tem-se que $\left|\lambda_{i}^{\mathrm{M}}\right|<1, i=1, \ldots, m$, se $\sigma \in(0,2)$; e $\left|\lambda_{i}^{\mathrm{M}}\right| \geq 1$, para algum $i$ se $\sigma \in[2, \infty)$ o que completa a prova do teorema 10 . 


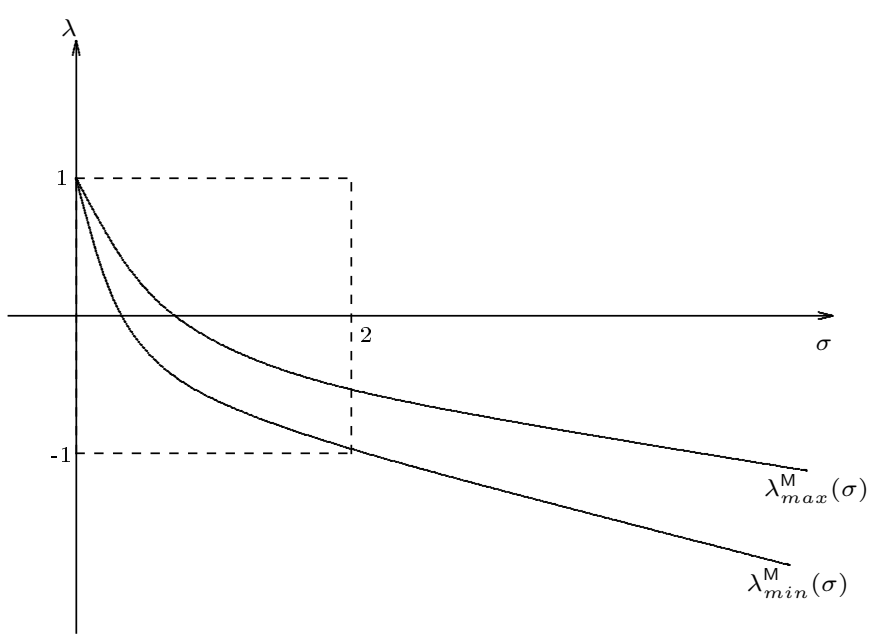

Figura 3.6: Ilustração do comportamento de $\lambda_{\max }^{\mathrm{M}}(\sigma)$ e $\lambda_{\min }^{\mathrm{M}}(\sigma)$.

\subsection{Verificação}

Os esquemas analisados neste capítulo, com condições de contorno tratadas explicitamente e implicitamente, serão testados para as equações de Navier-Stokes, com o objetivo de ilustrar numericamente o comportamento destes métodos.

O problema teste empregado foi o escoamento de um fluido newtoniano entre duas placas paralelas, em um canal de largura $L$ e comprimento $5 L$. Este escoamento é conhecido como de Hagen-Poiseuille, e é caracterizado pelo fato de que o canal está inicialmente cheio e portanto não há superfície livre, e denotado aqui como escoamento confinado. A solução analítica pode ser verificada em Batchelor [10].

As condições iniciais e de contorno para a solução das equações de Navier-Stokes (2.1) e (2.2) foram apresentadas no capítulo 2. Em particular, para esta simulação foi aplicado a condição de contorno no-slip (2.4) para o contorno rígido, isto é, as componentes normal e tangencial da velocidade são iguais a zero. No injetor aplicou-se as condições de contorno (2.6) e nos ejetores condições de contorno do tipo Neumann homogênea.

A modelagem, simulação e visualização dos testes foram realizados no sistema FREEFLOW [15]. Os testes foram processados em um Dual Atlon XP MP $2200 \mathrm{MHz}$ com 1Gb de memória RAM. Em todas as simulações utilizou-se uma malha estruturada uniforme, e nas soluções dos sistemas lineares utilizou-se uma tolerância $\epsilon=1.0 \times 10^{-10}$ para os testes de convergência.

Nestas simulações foram utilizados dois números de Reynolds a saber $R e=0.1$ e $R e=0.01$, obtidos a partir dos seguintes dados: $\nu=10.0 \mathrm{~m}^{2} \mathrm{~s}^{-1}, \nu=100.0 \mathrm{~m}^{2} \mathrm{~s}^{-1}, U=1.0 \mathrm{~ms}^{-1}$ e $L=$ 
$1.0 \mathrm{~m}$. Assumiu-se $\delta x=\delta y=0.05 \mathrm{~m}$, e as equações foram integradas de $t=0 \mathrm{~s}$ até $t=20 \mathrm{~s}$. $\mathrm{O}$ erro relativo foi calculado na norma $l_{2}$. A comparação entre a solução numérica e a solução exata foi realizada em $x=\frac{5 L}{2}$.

Os métodos testados foram: o método explícito GENSMAC [163] utilizando a equação (2.25); o método de Euler implícito (EI) utilizando a equação (2.37) e o método de CrankNicolson $(\mathrm{CN})$ utilizando a equação (2.38).

Utiliza-se a seguinte notação: GENSMAC/CCE para denotar o método GENSMAC com Condições de Contorno Explícitas (CCE); EI/CCE para o esquema de Euler implícito com condições de contorno explícitas; CN/CCE para o método de Crank-Nicolson com condições de contorno explícitas; CN/CCI1 para o método de Crank-Nicolson com Condições de Contorno Implícitas (CCI) do tipo 1 e CN/CCI2 para o tipo 2.

Os resultados são apresentados nas tabelas 3.1 e 3.2 para o problema confinado. Nestas tabelas, Er é o erro relativo na norma $l_{2}$ e $\delta t$ é o passo temporal adimensional.

Tabela 3.1: Escoamento confinado $(R e=0.1)$.

\begin{tabular}{|c|c|c|c|}
\hline Método & $E r(u(y))$ & $\delta t$ & $\begin{array}{c}\text { Tempo de processamento } \\
\text { (minutos }: \text { segundos) }\end{array}$ \\
\hline GENSMAC/CCE & $1.5099 \times 10^{-3}$ & $2.5 \times 10^{-5}$ & $104: 31$ \\
EI/CCE & $1.5185 \times 10^{-3}$ & $1.25 \times 10^{-2}$ & $3: 19$ \\
$\mathrm{CN} / \mathrm{CCI} 1$ & $1.5165 \times 10^{-3}$ & $1.25 \times 10^{-2}$ & $3: 05$ \\
$\mathrm{CN} / \mathrm{CCI} 2$ & $1.5137 \times 10^{-3}$ & $1.25 \times 10^{-2}$ & $3: 48$ \\
$\mathrm{CN} / \mathrm{CCE}$ & $1.5138 \times 10^{-3}$ & $5.0 \times 10^{-4}$ & $10: 28$ \\
\hline
\end{tabular}

Tabela 3.2: Escoamento confinado $(R e=0.01)$.

\begin{tabular}{|c|c|c|c|}
\hline Método & $E r(u(y))$ & $\delta t$ & $\begin{array}{c}\text { Tempo de processamento } \\
\text { (minutos : segundos) }\end{array}$ \\
\hline GENSMAC/CCE & $1.4817 \times 10^{-3}$ & $2.5 \times 10^{-6}$ & $874: 01$ \\
EI/CCE & $1.4182 \times 10^{-3}$ & $1.25 \times 10^{-2}$ & $3: 39$ \\
$\mathrm{CN} / \mathrm{CCI} 1$ & $1.4937 \times 10^{-3}$ & $1.25 \times 10^{-2}$ & $3: 19$ \\
$\mathrm{CN} / \mathrm{CCI} 2$ & $1.4937 \times 10^{-3}$ & $1.25 \times 10^{-2}$ & $4: 05$ \\
$\mathrm{CN} / \mathrm{CCE}$ & $1.5138 \times 10^{-3}$ & $5.0 \times 10^{-5}$ & $80: 20$ \\
\hline
\end{tabular}

As tabelas 3.1 e 3.2 indicam que o método de Crank-Nicolson com condições de contorno explícitas é condicionalmente estável. Este fato foi provado na seção 3.3 para a equação modelo, e agora verificado para as equações de Navier-Stokes. Como pode ser observado nas tabelas 3.1 e 3.2, o passo temporal usado pelo método explícito GENSMAC é o menor de todos os 
outros, por causa da restrição de estabilidade parabólica imposta em esquemas explícitos (veja restrição $(2.34))$.

O método de Euler implícito com condições de contorno explícitas e o método de CrankNicolson com condições de contorno implícitas, do tipo 1 e tipo 2, são incondicionalmente estáveis. Estes fatos também foram provados na seção 3.3 para o problema modelo. Consequentemente, o tempo de processamento utilizado por estes esquemas foi consideravelmente inferior aos dos demais esquemas.

\subsection{Considerações finais}

Neste capítulo, o principal resultado foi a demonstração de que o método de Crank-Nicolson pode tornar-se condicionalmente estável dependendo de como as condições de contorno são discretizadas. Esse resultado parece ser inédito. Recentemente, utilizando uma análise do tipo von Neumann, Tadjeran [147] apresentou um estudo do método de Crank-Nicolson para a equação do calor em problemas com coeficiente de difusão transiente, demonstrando que é necessário realizar uma média temporal no cálculo do coeficiente para que o método torne-se incondicionalmente estável. Entretanto, na análise de Tadjeran [147], as condições de contorno não foram incluídas na análise.

Além dos estudos sobre o método de Crank-Nicolson, os esquemas de Euler explícito e implícito também foram analisados para condições de contorno explícitas e implícitas. Os resultados destas análises confirmam resultados já apresentados na literatura. A estabilidade dos esquemas numéricos foi estudada por meio dos autovalores da matriz de iteração aplicando teoremas clássicos de algebra linear, além de um teorema recente de Yueh [180]. Finalmente, concluiu-se que as restrições de estabilidade derivadas para o problema modelo descrito neste capítulo são aparentemente válidas também para as equações de Navier-Stokes, como observado nos testes numéricos. Os resultados deste capítulo foram publicados em Oishi et al. [117]. 


\section{Métodos de projeção para escoamentos com superfície livre: caso bidimensional}

Como comentado anteriormente, muitos trabalhos já foram apresentados utilizando o método MAC. Entretanto, poucos combinam a formulação MAC para escoamentos com superfície livre a baixo número de Reynolds com métodos de projeção, que é o foco principal deste trabalho.

A exemplo dos capítulos anteriores, uma pergunta é proposta ao leitor: "Como construir um método de projeção estável e consistente para escoamentos com superfície livre ?" O objetivo deste capítulo é responder esta questão.

\subsection{Introdução}

No capítulo 2 foram apresentados alguns conceitos básicos em escoamentos com superfície livre. A formulação apresentada do método GENSMAC é baseada em um método de projeção. Neste capítulo, duas novas metodologias serão apresentadas para a simulação numérica de escoamentos com superfície livre a baixo número de Reynolds. A primeira é baseada na aplicação do método de Euler implícito para a solução da equação de quantidade de movimento (2.22). A originalidade desta primeira metodologia está no tratamento da pressão na superfície livre: uma técnica implícita para a solução da equação da pressão na superfície livre (2.7) combinada com a equação (2.37) resultará em um novo método de projeção para superfície livre. A segunda 
metodologia é baseada na anterior, porém utilizando o método de Crank-Nicolson combinado com uma nova fórmula para atualização da pressão. A motivação para a construção destas novas formulações pode ser resumida em dois pontos:

- Possibilitar que o pacote computacional FREEFLOW simule problemas reais em tempo razoável;

- Apresentar novas técnicas numéricas baseadas em métodos de projeção para escoamentos com superfície livre a baixo número de Reynolds.

Um objetivo fundamental deste trabalho é estudar e avaliar o uso de métodos implícitos no FREEFLOW encorajado pelos bons resultados apresentados em [113]. Muitas simulações para escoamentos com superfície livre foram realizadas utilizando o método explícito GENSMAC do sistema FREEFLOW. Entretanto, como já comentado anteriormente, a aplicação do esquema explícito resulta numa restrição do tamanho do passo temporal, e consequentemente o tempo de processamento utilizado do FREEFLOW pode tornar-se inaplicável em certos problemas. Até recentemente, o sistema FREEFLOW não disponibilizava a opção por técnicas implícitas, e alguns problemas interessantes de caráter acadêmico e industrial não puderam ser simulados. Neste projeto de doutorado, variações dos métodos de projeção e técnicas implícitas foram implementadas e testadas no sistema FREEFLOW. Neste capítulo, duas destas variações serão apresentadas.

Um objetivo acadêmico deste capítulo é introduzir novas idéias sobre métodos de projeção para escoamentos com superfície livre. Como relatado por Guermond et al. [61], existem poucos resultados direcionados à construção de métodos de projeção que utilizem corretamente as condições de contorno na superfície livre. Além do trabalho de Guermond et al. [61], outro resultado interessante nesta linha para problemas bidimensionais foi apresentado por Yang e Prosperetti [177]. Apesar de conter um estudo teórico abrangente, os trabalhos de Guermond et al. e Yang e Prosperetti não apresentaram simulações para problemas com superfície livre e baixo número de Reynolds. Além disso, os resultados em [61, 177] são apenas bidimensionais.

Analisando os dois pontos acima, percebe-se a necessidade de combinar novos métodos de projeção para escoamentos com superfície livre e baixo número de Reynolds com o sistema FREEFLOW. A seguir, apresenta-se os detalhes das novas formulações propostas neste trabalho. 


\subsection{Um método semi-implícito estável utilizando EI}

Uma discretização de primeira ordem para as equações de Navier-Stokes, baseada no método de Euler implícito (EI), é obtida utilizando $\theta_{1}=\theta_{2}=1, \theta_{3}=\theta_{4}=0$ e $\alpha=1$ na equação (2.17), ou seja a discretização

$$
\frac{\mathbf{u}^{(n+1)}}{\delta t}-R e^{-1} \nabla^{2} \mathbf{u}^{(n+1)}=\frac{\mathbf{u}^{(n)}}{\delta t}-\nabla \cdot(\mathbf{u u})^{(n)}-\nabla p^{(n+1)}+\left(F r^{2}\right)^{-1} \mathbf{g}^{(n)},
$$

juntamente com a equação da continuidade

$$
\nabla \cdot \mathbf{u}^{(n+1)}=0
$$

Esta discretização é conhecida como semi-implícita, pois trata os termos viscosos implicitamente, enquanto que os termos convectivos são tratados explicitamente. É fácil verificar que as equações (4.1) e (4.2) acoplam os campos de velocidade e pressão, e portanto, como já discutido anteriormente na seção 2.6, um desacoplamento deve ser aplicado, de tal forma que estas equações possam ser resolvidas separadamente.

Uma nova formulação será apresentada a seguir e tem por objetivo tornar o método semiimplícito mais estável. Para isso, uma nova forma de tratar a condição de contorno para a pressão na superfície livre deve ser encontrada. Considere o caso bidimensional e a condição de contorno na superfície livre dada pela equação (2.11). O primeiro passo na construção desta formulação é a discretização implícita desta equação, isto é

$$
-p^{(n+1)}+\frac{2}{R e}\left[\frac{\partial u}{\partial x} n_{x}^{2}+\frac{\partial v}{\partial y} n_{y}^{2}+\left(\frac{\partial u}{\partial y}+\frac{\partial v}{\partial x}\right) n_{x} n_{y}\right]^{(n+1)}=0 \quad \text { em } \quad \partial \Omega_{4} .
$$

Note que a equação (4.3) acopla os campos de velocidade e de pressão, e a solução do sistema acoplado pelas equações (4.1), (4.2) e (4.3) é indesejável, pois este sistema linear de equações algébricas será muito extenso, podendo apresentar ainda não-linearidades (como comentado na seção 2.6). Portanto, da mesma forma que é necessário desacoplar as equações (4.1) e (4.2), neste passo será necessário desenvolver uma estratégia para desacoplar os campos de velocidade e pressão na equação (4.3). Esta estratégia utiliza a equação (2.27) para a atualização da velocidade e a equação (2.32) para a atualização da pressão para construir novas equações para a pressão provisória $\psi^{(n+1)}$ na superfície livre.

Para deduzir essas equações, considere primeiro o caso em que uma célula [S] da superfície livre está em contato com uma célula $[\mathbf{E}]$ como representado na figura 2.4a). Neste caso o vetor 
normal é $\mathbf{n}=(1,0)$ e a equação (4.3) reduz-se a

$$
p^{(n+1)}=\frac{2}{R e}\left(\frac{\partial u^{(n+1)}}{\partial x}\right) .
$$

Da equação da continuidade (4.2) tem-se

$$
\frac{\partial u^{(n+1)}}{\partial x}=-\frac{\partial v^{(n+1)}}{\partial y}
$$

que quando substituída na equação (4.4) torna-se

$$
p^{(n+1)}=-\frac{2}{R e}\left(\frac{\partial v^{(n+1)}}{\partial y}\right) .
$$

De acordo com a equação (2.27) a velocidade final na direção $y$ discretizada no nível de tempo $(n+1)$ é dada por

$$
v^{(n+1)}=\widetilde{v}-\delta t \frac{\partial \psi^{(n+1)}}{\partial y} .
$$

Substituindo (4.7) em (4.6) tem-se

$$
p^{(n+1)}=-\frac{2}{R e}\left(\frac{\partial \widetilde{v}}{\partial y}-\delta t \frac{\partial^{2} \psi^{(n+1)}}{\partial y^{2}}\right) .
$$

Finalmente, substituindo (2.32) na equação (4.8) obtém-se

$$
\psi^{(n+1)}-\frac{2 \delta t}{R e}\left(\frac{\partial^{2} \psi^{(n+1)}}{\partial y^{2}}\right)=-\frac{2}{R e}\left(\frac{\partial \widetilde{v}}{\partial y}\right)-p^{(n)}
$$

Esta equação é usada para definir $\psi$ na superfície livre para o caso descrito na figura 2.4a).

O segundo caso é dado pela configuração da figura 2.6a) em que $\mathbf{n}=\left(\frac{\sqrt{2}}{2}, \frac{\sqrt{2}}{2}\right)$, e desta forma a equação (4.3) é reescrita como

$$
p^{(n+1)}=\frac{1}{R e}\left(\frac{\partial u^{(n+1)}}{\partial y}+\frac{\partial v^{(n+1)}}{\partial x}\right) .
$$

Da equação (2.27) o campo de velocidade na direção $x$ discretizado no nível de tempo $(n+1)$ é

$$
u^{(n+1)}=\widetilde{u}-\delta t \frac{\partial \psi^{(n+1)}}{\partial x}
$$


Substituindo as equações (4.11) e (4.7) em (4.10) tem-se

$$
p^{(n+1)}=\frac{1}{R e}\left(\frac{\partial \widetilde{u}}{\partial y}-\delta t \frac{\partial^{2} \psi^{(n+1)}}{\partial x \partial y}+\frac{\partial \widetilde{v}}{\partial x}-\delta t \frac{\partial^{2} \psi^{(n+1)}}{\partial x \partial y}\right),
$$

que substituindo em (2.32) torna-se

$$
\psi^{(n+1)}+\frac{2 \delta t}{R e}\left(\frac{\partial^{2} \psi^{(n+1)}}{\partial x \partial y}\right)=\frac{1}{R e}\left(\frac{\partial \widetilde{u}}{\partial y}+\frac{\partial \widetilde{v}}{\partial x}\right)-p^{(n)} .
$$

Esta equação é utilizada para definir $\psi$ na superfície livre para o caso descrito na figura 2.6a).

A construção da equação para $\psi$ nos casos apresentados nas figuras 2.4b) e 2.5, e para os outros casos descritos na figura 2.6 é muito semelhante, e não será apresentada.

Portanto, combinando a solução da equação de quantidade de movimento discretizada pelo método de Euler implícito com as novas equações para $\psi$ na superfície livre foi possível construir um método de projeção para escoamentos com superfície livre. O procedimento de solução para este método será apresentada a seguir.

\subsubsection{Algoritmo}

O algoritmo da formulação descrita anteriormente segue os seguinte passos:

- Passo 1: Calcular o campo de velocidade intermediário $\widetilde{\mathbf{u}}$ da equação

$$
\frac{\widetilde{\mathbf{u}}}{\delta t}-R e^{-1} \nabla^{2} \widetilde{\mathbf{u}}=\frac{\mathbf{u}^{(n)}}{\delta t}-\nabla \cdot(\mathbf{u u})^{(n)}-\nabla p^{(n)}+\left(F r^{2}\right)^{-1} \mathbf{g}^{(n)}
$$

Note que a equação acima é a mesma que (2.37), e será repetida neste momento apenas para facilitar o entendimento do algoritmo.

As condições de contorno para $\widetilde{\mathbf{u}}$ nos contorno rígidos, injetor e ejetor são as mesmas de $\mathbf{u}^{(n+1)}$ como descrito no capítulo 2. As condições de contorno para $\widetilde{\mathbf{u}}$ na superfície livre $\left(\partial \Omega_{4}\right)$ são calculadas explicitamente pelas equações (2.2) e (2.12) como descrito no Apêndice A. Portanto, na solução da equação acima, quando necessita de $\widetilde{\mathbf{u}}$ na superfície livre assume-se

$$
\widetilde{\mathbf{u}}=\mathbf{u}^{(n)},
$$

onde $\mathbf{u}^{(n)}$ é calculado das condições (2.2) e (2.12). 
O sistema linear resultante da discretização implícita acima é esparso e simétrico, e é resolvido pelo método dos gradientes conjugados [142].

- Passo 2: Resolver a equação de Poisson

$$
\nabla^{2} \psi^{(n+1)}=\frac{1}{\delta t} \nabla \cdot \widetilde{\mathbf{u}}
$$

junto com as equações derivadas para a pressão tentativa $\psi^{(n+1)}$ na superfície livre (ver por exemplo as equações (4.9) e (4.13)). As condições de contorno apropriadas para resolver este conjunto de equações são

$$
\frac{\partial \psi}{\partial n}=0, \quad \text { em } \quad \partial \Omega_{1} \text { (contorno rígido) e } \partial \Omega_{2} \text { (injetor) }
$$

além de considerar $\psi^{(n+1)}=0$ em $\partial \Omega_{3}$ (ejetor). Note que a diferença principal entre esta formulação e a metodologia GENSMAC é que agora $\psi^{(n+1)}$ na superfície livre não é mais zero, como ocorre no GENSMAC. O sistema linear resultante para $\psi^{(n+1)}$ é esparso e nãosimétrico, e é resolvido pelo método dos gradientes bi-conjugados com o pré-condicionador de Jacobi [130].

- Passo 3: Atualizar o campo de velocidade final pela equação

$$
\mathbf{u}^{(n+1)}=\widetilde{\mathbf{u}}-\delta t \nabla \psi^{(n+1)} .
$$

- Passo 4: Atualizar o campo de pressão final pela equação

$$
p^{(n+1)}=p^{(n)}+\psi^{(n+1)} .
$$

- Passo 5: Determinar as novas posições das partículas marcadoras virtuais resolvendo as equações

$$
\dot{\mathbf{x}}=\mathbf{u}^{(n+1)} .
$$

Novamente neste passo, assim como no passo 6 do algoritmo do GENSMAC (ver subseção 2.7.1), utiliza-se o esquema de Euler explícito para resolver as equações acima. Detalhes da implementação deste esquema foram apresentados em Castelo et al. [15]. 


\subsection{Um método semi-implícito estável utilizando CN}

Para construir um método semi-implícito estável, e também mais preciso, considere a discretização temporal de segunda ordem para as equações de Navier-Stokes, baseada no método de Crank-Nicolson (CN), utilizando $\theta_{1}=\theta_{4}=0.5, \theta_{2}=1, \theta_{3}=0$ e $\alpha=1$ na equação (2.17), ou seja

$$
\frac{\mathbf{u}^{(n+1)}}{\delta t}-\frac{1}{2 \operatorname{Re}} \nabla^{2} \mathbf{u}^{(n+1)}=\frac{\mathbf{u}^{(n)}}{\delta t}+\frac{1}{2 R e} \nabla^{2} \mathbf{u}^{(n)}-\nabla \cdot(\mathbf{u u})^{(n)}-\nabla p^{(n+1)}+\left(F r^{2}\right)^{-1} \mathbf{g}^{(n)},
$$

com a equação da continuidade (4.2). Neste método, uma nova equação para a pressão é derivada. Para isso, considere a equação acima, que para a velocidade intermediária é dada por (2.38), apresentada na seção 2.8. Agora, introduzindo $\widetilde{u}$ dado pela equação (2.27) em (2.38) tem-se

$$
\begin{aligned}
\frac{\mathbf{u}^{(n+1)}+\delta t \nabla \psi^{(n+1)}}{\delta t} & -\frac{1}{2 R e} \nabla^{2}\left(\mathbf{u}^{(n+1)}+\delta t \nabla \psi^{(n+1)}\right)=\frac{\mathbf{u}^{(n)}}{\delta t}+\frac{1}{2 R e} \nabla^{2} \mathbf{u}^{(n)}-\nabla \cdot(\mathbf{u u})^{(n)} \\
& -\nabla p^{(n)}+\left(F r^{2}\right)^{-1} \mathbf{g}^{(n)}
\end{aligned}
$$

Quando a equação (4.16) é comparada com a equação (4.15), obtém-se

$$
p^{(n+1)}=p^{(n)}+\psi^{(n+1)}-\frac{\delta t}{2 R e} \nabla^{2} \psi^{(n+1)} .
$$

Portanto, o método semi-implícito estável, agora utiliza o esquema de Crank-Nicolson para construir uma nova fórmula para atualização da pressão (4.17). Esta forma de atualizar a pressão foi investigada por Brown et al. [13] e Guermond et al. [61], que analisaram diferentes fórmulas de corrigir a pressão.

Novamente, na formulação semi-implícita utilizando o método Crank-Nicolson, é necessário discretizar implicitamente a equação da pressão na superfície livre, como foi feito na formulação anterior. Portanto, considere a equação bidimensional (4.3) novamente. Da mesma forma como apresentada na seção 4.2, a equação (4.3) será utilizada para construir equações para $\psi^{(n+1)}$ na superfície livre.

Considere a equação (4.8) que foi derivada na seção 4.2. Para esta nova formulação, a única diferença é que agora, deve-se substituir (4.17) em (4.8), e assim obtém-se

$$
\psi^{(n+1)}-\frac{\delta t}{2 R e} \nabla^{2} \psi^{(n+1)}-\frac{2 \delta t}{R e}\left(\frac{\partial^{2} \psi^{(n+1)}}{\partial y^{2}}\right)=-\frac{2}{R e}\left(\frac{\partial \widetilde{v}}{\partial y}\right)-p^{(n)} .
$$


Logo, esta é a equação a ser utilizada para $\psi^{(n+1)}$ na superfície livre para o caso representado na figura $2.4 \mathrm{a})$.

Assim como na seção 4.2, o segundo caso a ser analisado utiliza a equação (4.12). Agora, substituindo (4.17) em (4.12) tem-se

$$
\psi^{(n+1)}-\frac{\delta t}{2 R e} \nabla^{2} \psi^{(n+1)}+\frac{2 \delta t}{R e}\left(\frac{\partial^{2} \psi^{(n+1)}}{\partial x \partial y}\right)=\frac{1}{R e}\left(\frac{\partial \widetilde{u}}{\partial y}+\frac{\partial \widetilde{v}}{\partial x}\right)-p^{(n)}
$$

que é utilizada para definir $\psi$ na superfície livre para o caso descrito na figura 2.6a).

A construção das equações de $\psi$ para os casos descritos nas figuras 2.4b) e 2.5, e para os outros casos descritos na figura 2.6 é muito semelhante, e não será apresentada.

Logo, combinando a equação de quantidade de movimento discretiza pelo método de CrankNicolson com a nova fórmula da pressão (4.17), e com as novas equações para $\psi$ na superfície livre, constrói-se uma nova formulação. O procedimento de solução apresenta algumas diferenças da primeira formulação e será detalhado a seguir.

\subsubsection{Algoritmo}

- Passo 1: Calcular o campo de velocidade intermediário $\widetilde{\mathbf{u}}$ da equação

$$
\frac{\widetilde{\mathbf{u}}}{\delta t}-\frac{1}{2 R e} \nabla^{2} \widetilde{\mathbf{u}}=\frac{\mathbf{u}^{(n)}}{\delta t}+\frac{1}{2 R e} \nabla^{2} \mathbf{u}^{(n)}-\nabla \cdot(\mathbf{u u})^{(n)}-\nabla p^{(n)}+\left(F r^{2}\right)^{-1} \mathbf{g}^{(n)}
$$

Note que a equação acima é a mesma que (2.38), e será repetida neste momento apenas para simplificar a leitura do algoritmo.

Novamente, as condições de contorno para $\widetilde{\mathbf{u}}$ são as mesmas que para $\mathbf{u}^{(n+1)}$ como apresentado no capítulo 2 para paredes rígidas, injetor e ejetor. Entretanto, como foi apresentado no capítulo 3, para conseguir que o método Crank-Nicolson seja incondicionalmente estável em uma malha deslocada é necessário que as condições para $\widetilde{\mathbf{u}}$ no contorno rígido $\left(\partial \Omega_{1}\right)$ e no injetor $\left(\partial \Omega_{2}\right)$ sejam discretizadas implicitamente. Por exemplo, considere a situação descrita na figura 3.1, do capítulo 3 , e a equação de quantidade de movimento na direção y para calcular a velocidade intermediária $\widetilde{v}$. Neste caso, de acordo com a condição de contorno sem deslizamento (no-slip) (2.4) para a velocidade intermediária $\widetilde{v}$, tem-se que a velocidade no contorno é zero, isto é, $v_{b}=0$ (veja figura 3.1). Após uma simples interpolação, a condição de contorno implícita para $\widetilde{v}$ na equação acima na 
direção $y$ é

$$
\widetilde{v}_{0}=-\widetilde{v}_{1} \quad \text { e } \quad \widetilde{v}_{m+1}=-\widetilde{v}_{m}
$$

Assim como no algoritmo da formulação anterior (veja na subseção 4.2.1), as condições de contorno para $\widetilde{\mathbf{u}}$ na superfície livre $\left(\partial \Omega_{4}\right)$ são calculadas explicitamente pelas equações (2.2) e (2.12). Ou seja, na solução da equação de quantidade de movimento acima, quando necessita de $\widetilde{\mathbf{u}}$ na superfície livre, assume-se simplesmente (4.14).

Novamente, o sistema linear resultante é esparso e simétrico, e é resolvido pelo método dos gradientes conjugados [142]. Note que, a modificação da condição de contorno implícita (4.20) não altera a estrutura simétrica da matriz, mas apenas fortalece o elemento da diagonal principal.

- Passo 2: Resolver a equação de Poisson

$$
\nabla^{2} \psi^{(n+1)}=\frac{1}{\delta t} \nabla \cdot \widetilde{\mathbf{u}}
$$

juntamente com as equações derivadas para a pressão tentativa $\psi^{(n+1)}$ na superfície livre (ver por exemplo as equações (4.18) e (4.19)). As demais condições de contorno para resolver este conjunto de equações foram apresentadas no passo 2 do algoritmo anterior (ver subseção 4.2.1). Da mesma forma que na formulação anterior, o sistema linear resultante para $\psi^{(n+1)}$ é esparso e não-simétrico, e é resolvido pelo método dos gradientes bi-conjugados com o pré-condicionador de Jacobi [130].

- Passo 3: Atualizar o campo de velocidade final pela equação

$$
\mathbf{u}^{(n+1)}=\widetilde{\mathbf{u}}-\delta t \nabla \psi^{(n+1)} .
$$

- Passo 4: Atualizar o campo de pressão final pela equação (4.17).

- Passo 5: Idem ao passo 5 da formulação anterior (veja na subseção 4.2.1).

\subsection{Verificação}

Com o objetivo de verificar os métodos de projeção para problemas com superfície livre em escoamentos bidimensionais newtonianos descritos neste capítulo, utiliza-se o mesmo problema teste apresentado no capítulo anterior: o escoamento de um fluido newtoniano em um canal 
de largura $L$ e comprimento $5 L$. Entretanto, agora o canal está inicialmente vazio e o fluido é injetado na entrada do canal com um perfil de velocidade parabólico. Neste caso, a superfície livre se movimenta ao longo do canal, e este problema apresenta um escoamento com formação do fountain flow.

As condições iniciais e de contorno para a solução das equações (2.1) e (2.2) são as mesmas que aquelas da seção 3.4 do capítulo anterior. Além destas condições, na superfície livre foi imposta a condição (2.11) para a pressão e (2.12) para a velocidade. Os equipamentos utilizados nos testes deste capítulo são os mesmos que do capítulo 3, seção 3.4.

Nestas simulações foram utilizados dois números de Reynolds a saber $R e=0.1$ e $R e=0.01$, obtidos a partir dos seguintes dados: $\nu=10.0 \mathrm{~m}^{2} \mathrm{~s}^{-1}, \nu=100.0 \mathrm{~m}^{2} \mathrm{~s}^{-1}, U=1.0 \mathrm{~ms}^{-1}$ e $L=$ $1.0 \mathrm{~m}$. Assumiu-se $\delta x=\delta y=0.05 \mathrm{~m}$, e as equações foram integradas de $t=0 \mathrm{~s}$ até $t=20 \mathrm{~s}$. O erro relativo foi calculado na norma $l_{2}$. A comparação entre a solução numérica e a solução exata foi realizada em $x=\frac{5 L}{2}$.

Nesta seção foram verificados o método de projeção com Euler implícito (EI), descrito na seção 4.2, e a segunda versão deste método utilizando o esquema de Crank-Nicolson (CN), apresentada na seção 4.3. Como descrito neste capítulo, ambas as formulações utilizam uma estratégia semi-implícita (SI), e portanto a notação utilizada será SI/EI para a primeira formulação, e SI/CN para a segunda.

Os resultados do capítulo anterior, evidenciam que a utilização do método CN requer um tratamento implícito das condições de contorno nas fronteiras rígidas. Logo, para o caso do SI/CN aplicou-se as CCI1 (veja detalhes na seção 3.4 do capítulo anterior), e para o método de EI apenas as CCE.

O método GENSMAC foi brevemente descrito na seção 2.7, enquanto que a sua modificação com métodos implícitos, denominado aqui de GENSMAC modificado, foi descrita na seção 2.8. Oishi et al. [114] mostraram que o método de Euler implícito, aplicado na formulação GENSMAC, é incondicionalmente estável para problemas confinados. Entretanto, para problemas com superfícies livres este esquema é ainda sujeito à condição de estabilidade parabólica, como no esquema explícito. Desta forma, nesta seção foi também verificado o esquema GENSMAC implementado com os métodos de EI e CN, como apresentado na seção 2.8 .

As tabelas 4.1 e 4.2 mostram que os métodos tipo GENSMAC são condicionalmente estáveis para escoamentos com superfície livre, inclusive quando aplicados com os métodos EI/CCE e CN/CCI1 na formulação. A instabilidade mostrada neste caso, não é devida ao tratamento explícito ou implícito das condições de contorno nas paredes rígidas, mas devida ao tratamento 
explícito das condições de contorno na superfície livre. Como pode ser notado das tabelas 4.1 e 4.2, os esquemas do tipo GENSMAC apresentam resultados semelhantes em todos os casos. O passo temporal usado por GENSMAC, GENSMAC-EI/CCE e GENSMAC-CN/CCI1 foi muito pequeno, e conseqüentemente, o tempo de processamento foi muito alto.

Logo, deduz-se das tabelas 4.1 e 4.2 que utilizar esquemas implícitos na equação de quantidade de movimento na formulação GENSMAC não é suficiente para eliminar a restrição de estabilidade parabólica (2.34). Portanto, o tratamento implícito da equação da pressão na superfície livre, apresentado neste capítulo, é fundamental.

Os esquemas SI são mais estáveis como pode ser observado nas tabelas 4.1 e 4.2. Como o passo no tempo do GENSMAC é muito pequeno, o erro relativo de todos os esquemas são muito próximos. Nas tabelas 4.1 e 4.2 , o erro relativo é calculado na norma $l_{2}$ e o passo temporal é adimensional.

Tabela 4.1: Escoamento com superfície livre $(R e=0.1)$.

\begin{tabular}{|c|c|c|c|}
\hline Método & $E r(u(y))$ & $\delta t$ & $\begin{array}{c}\text { Tempo de processamento } \\
\text { (minutos }: \text { segundos) }\end{array}$ \\
\hline GENSMAC & $1.5137 \times 10^{-3}$ & $2.5 \times 10^{-5}$ & $104: 40$ \\
GENSMAC-EI/CCE & $1.5139 \times 10^{-3}$ & $8.75 \times 10^{-5}$ & $60: 08$ \\
GENSMAC-CN/CCI1 & $2.9227 \times 10^{-3}$ & $5.0 \times 10^{-5}$ & $95: 58$ \\
SI-EI/CCE & $1.5158 \times 10^{-3}$ & $1.25 \times 10^{-2}$ & $3: 20$ \\
SI-CN/CCI1 & $1.5138 \times 10^{-3}$ & $1.25 \times 10^{-2}$ & $3: 05$ \\
\hline
\end{tabular}

Tabela 4.2: Escoamento com superfície livre $(R e=0.01)$.

\begin{tabular}{|c|c|c|c|}
\hline Método & $E r(u(y))$ & $\delta t$ & $\begin{array}{c}\text { Tempo de processamento } \\
\text { (minutos : segundos) }\end{array}$ \\
\hline GENSMAC & $1.4803 \times 10^{-3}$ & $2.5 \times 10^{-6}$ & $875: 16$ \\
GENSMAC-EI/CCE & $1.5009 \times 10^{-3}$ & $8.5 \times 10^{-6}$ & $544: 55$ \\
GENSMAC-CN/CCI1 & $2.8673 \times 10^{-3}$ & $4.75 \times 10^{-6}$ & $822: 15$ \\
SI-EI/CCE & $1.5151 \times 10^{-3}$ & $7.5 \times 10^{-4}$ & $25: 54$ \\
SI-CN/CCI1 & $1.5137 \times 10^{-3}$ & $5.5 \times 10^{-4}$ & $28: 59$ \\
\hline
\end{tabular}

Além destes resultados, um estudo da convergência dos métodos SI-EI e SI-CN também foi realizado para o escoamento fountain flow. Novamente, neste caso simples é possível comparar a solução numérica com a solução exata (ver em [10]). Ambas formulações foram aplicadas em três malhas: M1 $-50 \times 10$ células $\left(\delta x_{M 1}=0.1 m\right)$, M2 $-100 \times 20$ células $\left(\delta x_{M 2}=0.05 m\right)$ e M3 - $200 \times 40$ células $\left(\delta x_{M 3}=0.025 m\right)$. Nesta simulação assumiu-se $R e=0.1$, e a comparação entre a solução numérica e a solução exata foi realizada em $x=\frac{5 L}{2}$. 
Os passos temporais adimensionais utilizados para ambas as formulações foram $\delta t_{1}=5 \times$ $10^{-2}$ para a malha M1, $\delta t_{2}=1.25 \times 10^{-2}$ para a malha M2 e $\delta t_{3}=6.25 \times 10^{-3}$ para a malha M3.

Para demonstrar a convergência dos métodos, calculou-se o erro relativo na norma $l_{2}$ e a ordem $\left(N_{i}\right)$ pela fórmula

$$
N_{i}=\frac{\log \left(\frac{E r(\Gamma)_{M_{i+1}}}{\operatorname{Er}(\Gamma)_{M_{i}}}\right)}{\log \left(\frac{\delta x_{M_{i+1}}}{\delta x_{M_{i}}}\right)}, i=1,2,
$$

em que $M_{i}$ é a malha utilizada na simulação e $\Gamma=u(y)$. Os resultados são apresentados na tabela 4.3. De acordo com esta tabela, é possível verificar que a formulação SI-CN/CCI1 apresentou melhores resultados nos erros $E r$, e consequentemente a ordem média $N_{m}$ foi superior. Como os passos temporais utilizados, nas diferentes malhas, foram iguais nas duas formulações, o tempo de processamento foi muito semelhante e não foi apresentado na tabela 4.3.

Tabela 4.3: Escoamento com superfície livre $(R e=0.1)$, utilizando as malhas M1, M2 e M3. $N_{m}=\frac{N_{1}+N_{2}}{2}$.

\begin{tabular}{|c|c|c|c|c|c|c|}
\hline Método & $\operatorname{Er}(u(y))_{M 1}$ & $N_{1}$ & $\operatorname{Er}(u(y))_{M 2}$ & $N_{2}$ & $\operatorname{Er}(u(y))_{M 3}$ & $N_{m} \simeq$ \\
\hline SI-EI/CCE & $5.830 \times 10^{-3}$ & 1.62 & $1.515 \times 10^{-3}$ & 1.94 & $4.988 \times 10^{-4}$ & 1.78 \\
SI-CN/CCI1 & $5.001 \times 10^{-3}$ & 1.72 & $1.513 \times 10^{-3}$ & 1.97 & $3.856 \times 10^{-4}$ & 1.84 \\
\hline
\end{tabular}

A segunda formulação, SI-CN/CCI1, foi verificada para o problema com solução transiente do escoamento fountain flow. Nesta simulação, utilizou-se o mesmo modelo do canal formado por placas paralelas definidas no início da seção. A malha utilizada foi a M2 $\left(\delta x_{M 2}=0.05 m\right)$ enquanto que o número de Reynolds foi $R e=0.01$, baseado nos valores de $\nu=100.0 \mathrm{~m}^{2} \mathrm{~s}^{-1}$, $U=1.0 \mathrm{~ms}^{-1}$ e $L=1.0 \mathrm{~m}$. A solução exata para este escoamento, obtida pela aplicação das transformadas de Laplace (veja mais detalhes em [148, 104]), é

$$
u(x, y, t)=-4 y(y-1)-\frac{32}{\pi^{3}} \sum_{n=0}^{\infty}(2 n+1)^{3} \operatorname{sen}(y \pi(2 n+1)) \exp \left(-\operatorname{Re}^{-1}(2 n+1)^{2} \pi^{2} t\right),
$$

em que $x$ é a coordenada na direção do escoamento e $y$ é a coordenada na direção normal ao canal. No injetor, a condição inicial foi obtida de (4.22) com $t=0$. A figura 4.1 apresenta uma comparação entre o perfil da velocidade numérica transiente $u(y, t)$ e a solução exata (4.22), no ponto $x=\delta x_{M 2}$ do canal. Considerou-se 1000 termos na série (4.22). Pode-se verificar nesta figura que os resultados desta simulação estão em concordância com a solução exata. No estágio inicial transiente, em $t=0.0005 \mathrm{~s}$, as soluções apresentam pequenas diferenças que desaparecem 
com o tempo. A figura 4.2 apresenta o perfil da superfície livre e o campo de velocidade $u$ no tempo $t=4 s$.

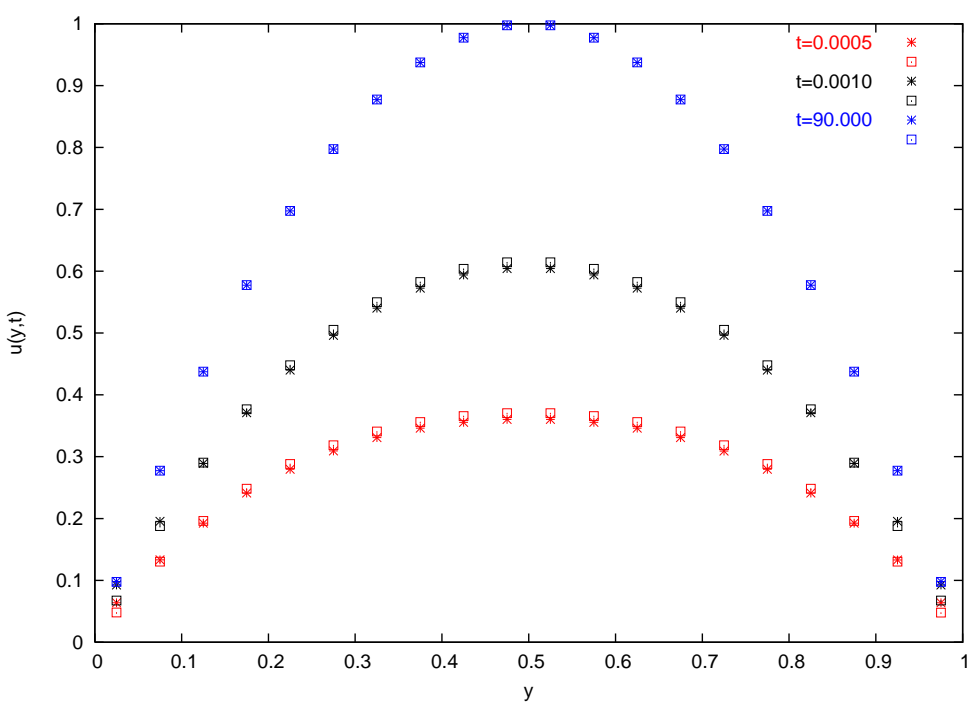

Figura 4.1: Comparação entre a solução numérica * e a solução exata $\square$ para o escoamento fountain flow em diferentes tempos utilizando a formulação SI-CN/CCI1.
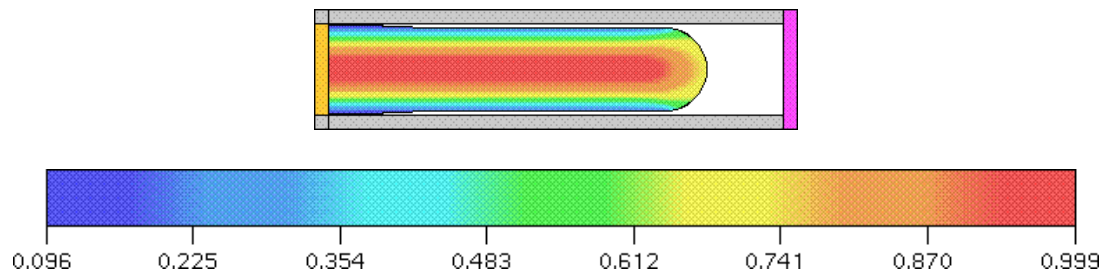

Figura 4.2: Perfil da superfície livre e distribuição da velocidade $u$ no tempo $t=4 \mathrm{~s}$ para o escoamento fountain flow.

\subsection{Aplicação}

As formulações descritas nas seções 4.2 e 4.3 foram verificadas em uma aplicação bidimensional: a simulação do problema do inchamento do extrudado de um fluido viscoso newtoniano. Apesar deste fenômeno ser mais interessante para escoamentos de fluidos viscoelásticos, como será apresentado posteriormente, este é um problema com superfície livre muito conhecido na literatura. Neste problema, para baixo número de Reynolds, o fluido tende a inchar quando sai de um canal e entra em contato com a atmosfera.

Para fluidos newtonianos muitos resultados foram apresentados ao longo dos anos, como por exemplo, os trabalhos experimentais de Tanner [149], Hill e Chenier [68], e os resultados numéricos de Tanner [150], Liu et al. [96], Ngamaramvaranggul e Webster [107], Housiadas et 
al. [74], entre outros. Muitos desses esquemas numéricos foram usados para resolver o problema do inchamento do extrudado axisimétrico, devido à existência de resultados experimentais para este caso. No caso de escoamento planar, resultados experimentais são mais difíceis de se obter e, conseqüentemente, apenas resultados numéricos têm sido apresentados na literatura $[150,18,119,28,39,30,29,1,159,54,53]$.

Um aspecto importante do problema do inchamento do extrudado é a influência do número de Reynolds e da tensão superficial. De acordo com Omodei [119], Tanner [150], Gifford [54], Housiadas et al. [74], esses parâmetros podem afetar o raio de inchamento. Neste trabalho, como comentado anteriormente, a tensão superficial é desprezada.

O raio do inchamento do extrudado $S_{r}$ (swelling ratio) no caso bidimensional é definido como

$$
S_{r}=\frac{L_{e}}{L}
$$

onde $L_{e}$ é a largura do jato extrudado e $L$ é a largura do canal (ver figura 4.3b)). Para problemas planares, vários valores de $S_{r}$ podem ser encontrados na literatura. Por exemplo, os seguintes autores obtiveram os valores: 1.155 (Chang et al. [18]), 1.161 (Dutta e Ryan [39]), 1.170 (Chang et al. [18]), 1.186 (Georgiou e Boudouvis [53]), 1.190 (Tanner [150] e Omodei [119]), 1.196 (Ahn e Ryan [1]), 1.200 e 1.227 (Crochet e Keunings [30]). De acordo com Tanner [150], a diferença entre os resultados está diretamente ligada aos métodos numéricos utilizados, a escolha da malha, e ainda aos valores do número de Reynolds e os efeitos da tensão superficial.

Novamente foi considerado o escoamento transiente de um fluido entre placas paralelas até a saída do canal, onde o fluido entra em contato com a atmosfera. Na entrada do canal foi considerada uma velocidade uniforme. As condições de contorno são dadas por (2.4), (2.6), (2.11) e (2.12). A geometria do escoamento para o problema do inchamento do extrudado é apresentado na figura 4.3 .

O coeficiente de viscosidade escolhido foi $\nu=100.0 \mathrm{~m}^{2} \mathrm{~s}^{-1}$, e os parâmetros de escala foram $L=1.0 \mathrm{~m}$ e $U=1.0 \mathrm{~ms}^{-1}$ resultando $R e=0.01$. As malhas utilizadas foram M2 $\left(\delta x_{M 2}=0.05\right)$ e M3 $\left(\delta x_{M 3}=0.025\right)$. Uma malha mais fina também foi utilizada M4 - $400 \times 80$ células $\left(\delta x_{M 4}=\right.$ $0.0125 \mathrm{~m})$. Nestas simulações, tem-se os passos temporais adimensionais $\delta t=5.25 \times 10^{-4}$ para $\mathrm{M} 2, \delta t=1.56 \times 10^{-4}$ para M3 e $\delta t=1.0 \times 10^{-5}$ para M4. A figura 4.4 mostra a configuração da superfície livre em tempos diferentes para a formulação SI-CN/CCI1 na malha M2.

A tabela 4.4 apresenta o efeito do refinamento de malha no raio do inchamento do extrudado e o passo temporal adimensional para as formulações SI-EI/CCE e SI-CN/CCI. Neste caso, o raio do inchamento do extrudado aumenta de 1.11 para 1.18 quando a malha é refinada. 


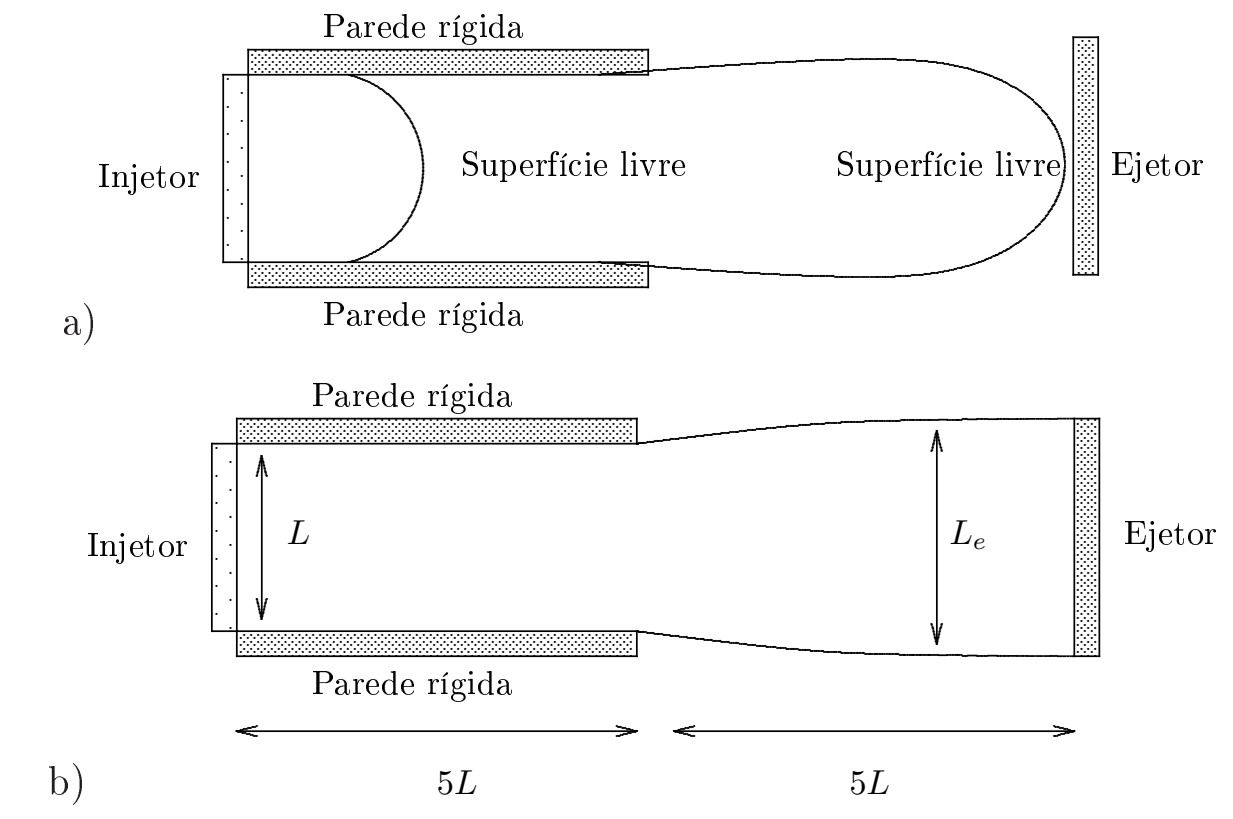

Figura 4.3: Geometria e contornos para o problema do inchamento do extrudado: a) estado transiente e b) estado estacionário.

a)

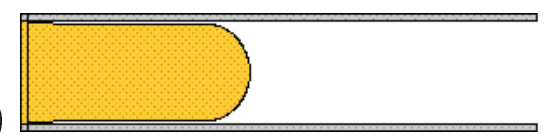

b)

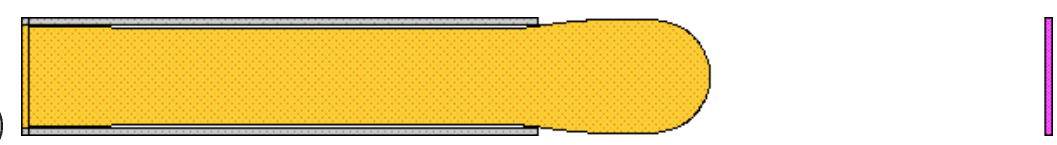

c)

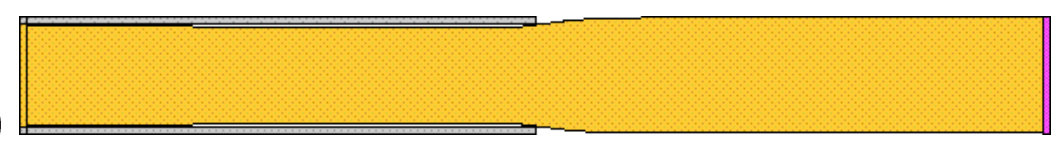

Figura 4.4: Perfil da superfície livre para o problema do inchamento do extrudado de um fluido newtoniano para a formulação SI-CN/CCI1 em diferentes tempos adimensionais: a) $t=3.0, \mathrm{~b}$ ) $t=9.8$ e c) $t=20.0$.

Tabela 4.4: Resultados do raio do inchamento do extrudado $S_{r}$ para $R e=0.01$ utilizando as formulações SI-EI/CCE e SI-CN/CCI1 em diferentes malhas (M2, M3 e M4).

\begin{tabular}{|c|c|c|c|}
\hline Método & M2 & M3 & M4 \\
\hline SI-EI/CCE & 1.115 & 1.150 & 1.182 \\
SI-CN/CCI1 & 1.120 & 1.157 & 1.182 \\
\hline
\end{tabular}


Métodos de projeção $2 D$

Os resultados do inchamento para ambas formulações estão razoavelmente de acordo com os resultados numéricos apresentados em $[18,53]$. Portanto, nota-se que os métodos de projeção para superfície livre descritos neste capítulo foram capazes de simular o efeito do inchamento do extrudado.

\subsection{Considerações finais}

Neste capítulo foram apresentadas propostas para a construção de métodos de projeção para escoamentos bidimensionais com superfície livre. Inicialmente, foi introduzido um método semi-implícito, baseado na discretização de Euler implícito, com um novo tratamento das condições de contorno na superfície livre. Em particular, a condição de tensão normal, que resulta em uma equação para a pressão na superfície livre, foi discretizada implicitamente. O resultado desta discretização foi o acoplamento dos campos de velocidade e pressão. Logo, uma nova metodologia foi descrita para desacoplar estes campos, e como conseqüência disto, foram construídas novas equações para a pressão tentativa $\psi$ na superfície livre. O método semiimplícito apresentado foi desenvolvido com o objetivo de superar a restrição de estabilidade parabólica derivada de esquemas explícitos, sem a necessidade de resolver simultaneamente os campos de velocidade e pressão. A dificuldade nesta formulação ocorre na solução do sistema linear envolvendo a equação de Poisson e as novas equações para $\psi$ na superfície livre. Este sistema é esparso, porém não-simétrico e o método dos gradientes bi-conjugados com précondicionamento de Jacobi foi aplicado. Apesar disto, o método é robusto e muito eficiente em simulações de escoamentos com superfície livre e baixo número de Reynolds como foi descrito nas seções 4.4 e 4.5 .

A segunda formulação apresentada é uma extensão da anterior, entretanto utiliza o método de Crank-Nicolson na discretização da equação de quantidade de movimento, resultando em uma nova fórmula de correção da pressão. Além disso, um ponto importante na utilização do método de Crank-Nicolson nesta formulação foi o tratamento implícito das condições de contorno em fronteiras rígidas. Esse tratamento é fundamental para manter o método estável como foi descrito no capítulo 3. Novamente, a equação para a pressão na superfície livre foi discretizada implicitamente, e a mesma idéia para o desacoplamento desta equação foi aplicada, derivando novas equações para a pressão tentativa $\psi$ na superfície livre. Assim como na primeira formulação, o método resultante é estável e permite a simulação de escoamentos a baixo número de Reynolds com o passo de tempo bem maior que o método explícito. Este fato também foi verificado nas seções 4.4 e 4.5. Os resultados deste capítulo foram publicados em Oishi et al. [116] e Ferreira et al. [48, 46]. 


\section{Métodos de projeção para problemas tridimensionais newtonianos e viscoelásticos com superfície livre}

Tendo desenvolvido os métodos de projeção para escoamentos com superfície livre no caso bidimensional, uma nova pergunta é proposta: "Como construir um método de projeção estável e consistente para problemas tridimensionais newtonianos e viscoelásticos? "Ao final deste capítulo, esta pergunta será respondida.

\subsection{Introdução}

Como comentado anteriormente, poucos trabalhos sobre métodos de projeção com superfície livre têm sido apresentados na literatura. Para problemas tridimensionais, ainda é mais escasso o número de trabalhos que discutem o assunto.

Exemplos de alguns destes trabalhos foram apresentados por Degani e Gutfinger [34], e recentemente por Perot e Nallapati [124] que apresentaram um esquema com movimentação de malha não-estruturada, Sousa et al. [145] que aplicaram um método de projeção para escoamentos multifásicos, Raad e Bidoae [135] que analisaram uma metodologia mista com técnicas eulerianas e lagrangeanas em escoamentos com superfície livre, Lin e Wang [93] que utilizaram um método de projeção em problemas de superfície livre com partículas, e Badiei et al. [8] que 
descreveram a construção de um método de volumes finitos baseado num método de projeção com superfície livre. Novamente, nestes trabalhos, não foram apresentados simulações a baixo número de Reynolds. Portanto, no contexto do método MAC e de escoamentos tridimensionais a baixo número de Reynolds, o estudo de métodos de projeção com superfície livre é muito motivador.

O objetivo deste capítulo é apresentar um método de projeção para escoamento tridimensional, utilizando as idéias descritas no capítulo anterior. Além de um método de projeção em escoamentos tridimensionais newtonianos, a sua extensão para escoamentos viscoelásticos será também apresentada.

\subsection{Extensão para problemas tridimensionais newtonianos e viscoelásticos}

Para estender as idéias descritas no capítulo anterior, seções 4.2 e 4.3, considere inicialmente as equações de Navier-Stokes

$$
\begin{gathered}
\frac{\partial \mathbf{u}}{\partial t}+\nabla \cdot(\mathbf{u u})=-\nabla p+\gamma \frac{1}{R e} \nabla^{2} \mathbf{u}+\beta \nabla \cdot \mathbf{S}+\frac{1}{F r^{2}} \mathbf{g}, \\
\nabla \cdot \mathbf{u}=0 .
\end{gathered}
$$

Note que a equação (5.1) é uma forma generalizada da equação (2.1) onde $\mathbf{S}$ é a contribuição não-newtoniana do tensor extra tensão que é definida por uma relação constitutiva apropriada. A equação (5.2) é a mesma que (2.2), e será repetida neste momento para facilitar a leitura deste capítulo.

Por simplicidade, $\mathbf{S}$ será denominado apenas como tensor tensão não-newtoniano. Em escoamentos newtonianos tem-se $\mathbf{S}=\mathbf{0}$, e então pode-se assumir $\gamma=1$ e $\beta=0$ na equação de quantidade de movimento (5.1). Neste trabalho, escoamentos viscoelásticos governados pelas equações constitutivas dos modelos diferencias de Maxwell e Oldroyd-B serão considerados. Para o modelo de Maxwell, assume-se $\gamma=\beta=1 \mathrm{em}$ (5.1), enquanto que para o modelo de Oldroyd-B tem-se $\gamma=\frac{\lambda_{2}}{\lambda_{1}}$ e $\beta=1$. Os coeficientes $\lambda_{1}$ e $\lambda_{2}$ são constantes temporais de relaxação e retardamento, respectivamente, caracterizando o modelo.

É importante mencionar que o objetivo desta seção é apresentar a extensão dos métodos semi-implícitos descritos anteriormente para problemas tridimensionais newtonianos e possíveis 
aplicações em problemas viscoelásticos. Neste trabalho não foi investigado com detalhes a equação e as condições de contorno para o tensor tensão não-newtoniano S. Quando se fez necessária a utilização dos termos que caracterizam um fluido viscoelástico, foram aplicadas as técnicas descritas em [143, 165] para o modelo Maxwell e em [156, 157] para o modelo OldroydB. Portanto, acreditando nos resultados apresentados em [156, 143, 165, 157], aplicam-se as ferramentas descritas nestes trabalhos para lidar com o tensor tensão não-newtoniano S no método de projeção que será descrito posteriormente.

De acordo com Silva e Tomé [143], o tensor tensão não-newtoniano S para o modelo Maxwell é obtido da equação

$$
\begin{aligned}
\frac{\partial \mathbf{S}}{\partial t} & =-\nabla \cdot(\mathbf{u S})+(\nabla \mathbf{u})^{T} \mathbf{S}+\mathbf{S}(\nabla \mathbf{u}) \\
& -\frac{1}{W e} \mathbf{S}-\frac{2}{R e}\left[\frac{\partial \mathbf{D}}{\partial t}+\nabla \cdot(\mathbf{u D})-(\nabla \mathbf{u})^{T} \mathbf{D}-\mathbf{D}(\nabla \mathbf{u})\right]
\end{aligned}
$$

onde $\mathbf{D}=\frac{1}{2}\left[\nabla \mathbf{u}+(\nabla \mathbf{u})^{T}\right]$. No caso do modelo Oldroyd-B, conforme os trabalhos de Tomé et al. $[156,157]$ tem-se

$$
\begin{aligned}
\frac{\partial \mathbf{S}}{\partial t} & =-\nabla \cdot(\mathbf{u S})+(\nabla \mathbf{u})^{T} \mathbf{S}+\mathbf{S}(\nabla \mathbf{u}) \\
& -\frac{1}{W e} \mathbf{S}+\frac{1}{W e}\left[\frac{1}{R e}\left(1-\frac{\lambda_{2}}{\lambda_{1}}\right)\left((\nabla \mathbf{u})+(\nabla \mathbf{u})^{T}\right)\right] .
\end{aligned}
$$

Nas equações acima $W e=\lambda_{1} \frac{U}{L}$ é o número de Weissenberg ( $U$ e $L$ são os parâmetros de escala).

As condições iniciais e de contorno na solução das equações de Navier-Stokes (5.1) e (5.2) foram apresentadas no capítulo 2. Para contornos em movimento, ou seja na superfície livre, o tensor de tensão cisalhante $\boldsymbol{\tau}$ é reescrito como

$$
\boldsymbol{\tau}=\gamma \frac{1}{R e}\left[\nabla \mathbf{u}+(\nabla \mathbf{u})^{T}\right]+\beta \mathbf{S}
$$

e consequentemente, o tensor total T, definido anteriormente por (2.9), é agora reescrito como

$$
\mathbf{T}=-p \mathbf{l}+\gamma \frac{1}{R e}\left[(\nabla \mathbf{u})+(\nabla \mathbf{u})^{\mathrm{T}}\right]+\beta \mathbf{S}
$$

onde, novamente, tem-se $\gamma=1$ e $\beta=0$ para escoamentos newtonianos, $\gamma=\beta=1$ para fluido do tipo Maxwell, e $\gamma=\lambda_{2} / \lambda_{1}$ e $\beta=1$ para fluido do tipo Oldroyd-B. Portanto, utilizando a nova forma do tensor total (5.6) nas equações (2.7), (2.8) e (2.13), constrói-se, respectivamente, 
as seguintes equações em forma cartesiana

$$
\begin{aligned}
& p=\gamma \frac{2}{R e}\left[\frac{\partial u}{\partial x} n_{x}^{2}+\frac{\partial v}{\partial y} n_{y}^{2}+\frac{\partial w}{\partial z} n_{z}^{2}+\left(\frac{\partial v}{\partial x}+\frac{\partial u}{\partial y}\right) n_{x} n_{y}\right. \\
& \left.+\left(\frac{\partial w}{\partial x}+\frac{\partial u}{\partial z}\right) n_{x} n_{z}+\left(\frac{\partial w}{\partial y}+\frac{\partial v}{\partial z}\right) n_{y} n_{z}\right] \\
& +\beta\left[S^{x x} n_{x}^{2}+S^{y y} n_{y}^{2}+S^{z z} n_{z}^{2}+2\left(S^{x y} n_{x} n_{y}+S^{x z} n_{x} n_{z}+S^{y z} n_{y} n_{z}\right)\right], \\
& 2 \frac{\partial u}{\partial x} n_{x} m_{x}+2 \frac{\partial v}{\partial y} n_{y} m_{y}+2 \frac{\partial w}{\partial z} n_{z} m_{z}+\left(\frac{\partial v}{\partial x}+\frac{\partial u}{\partial y}\right)\left(m_{x} n_{y}+m_{y} n_{x}\right) \\
& +\left(\frac{\partial w}{\partial x}+\frac{\partial u}{\partial z}\right)\left(m_{x} n_{z}+m_{z} n_{x}\right)+\left(\frac{\partial w}{\partial y}+\frac{\partial v}{\partial z}\right)\left(m_{y} n_{z}+m_{z} n_{y}\right) \\
& =-\frac{\beta}{\gamma} \operatorname{Re}\left[S^{x x} n_{x} m_{x}+S^{y y} n_{y} m_{y}+S^{z z} n_{z} m_{z}+S^{x y}\left(m_{x} n_{y}+m_{y} n_{x}\right)\right. \\
& \left.+S^{x z}\left(m_{x} n_{z}+m_{z} n_{x}\right)+S^{y z}\left(m_{y} n_{z}+m_{z} n_{x}\right)\right] \\
& 2 \frac{\partial u}{\partial x} n_{x} m_{1 x}+2 \frac{\partial v}{\partial y} n_{y} m_{1 y}+2 \frac{\partial w}{\partial z} n_{z} m_{1 z}+\left(\frac{\partial v}{\partial x}+\frac{\partial u}{\partial y}\right)\left(m_{1 x} n_{y}+m_{1 y} n_{x}\right) \\
& +\left(\frac{\partial w}{\partial x}+\frac{\partial u}{\partial z}\right)\left(m_{1 x} n_{z}+m_{1 z} n_{x}\right)+\left(\frac{\partial w}{\partial y}+\frac{\partial v}{\partial z}\right)\left(m_{1 y} n_{z}+m_{1 z} n_{y}\right) \\
& =-\frac{\beta}{\gamma} \operatorname{Re}\left[S^{x x} n_{x} m_{1 x}+S^{y y} n_{y} m_{1 y}+S^{z z} n_{z} m_{2 z}+S^{x y}\left(m_{1 x} n_{y}+m_{1 y} n_{x}\right)\right. \\
& \left.+S^{x z}\left(m_{1 x} n_{z}+m_{1 z} n_{x}\right)+S^{y z}\left(m_{1 y} n_{z}+m_{1 z} n_{x}\right)\right] .
\end{aligned}
$$

Portanto, as equações (5.7)-(5.9) são definidas em $\partial \Omega_{4}$, isto é na superfície livre. Detalhes sobre a construção destas equações podem ser encontrados em [156, 143, 157, 165]. A seguir a extensão dos métodos semi-implícitos descritos anteriormente será detalhada para escoamentos tridimensionais newtonianos e viscoelásticos.

Inicialmente, considere a discretização temporal da equação (5.1) da seguinte forma

$$
\begin{aligned}
\frac{\mathbf{u}^{(n+1)}}{\delta t}-\theta_{1} \frac{\gamma}{R e} \nabla^{2} \mathbf{u}^{(n+1)} & =\frac{\mathbf{u}^{(n)}}{\delta t}-\nabla \cdot(\mathbf{u u})^{(n)}-\nabla p^{(n+1)}+\theta_{4} \frac{\gamma}{R e} \nabla^{2} \mathbf{u}^{(n)} \\
& +\beta \nabla \cdot \mathbf{S}^{(n)}+\frac{1}{F r^{2}} \mathbf{g}^{(n)}
\end{aligned}
$$


juntamente com a equação da continuidade (5.2) discretizada no nível de tempo $(n+1)$. Na equação (5.10) os valores de $\theta_{1}$ e $\theta_{4}$, apresentados na tabela 2.1, definem o método implícito a ser utilizado, Euler implícito ou Crank-Nicolson. Como o objetivo deste trabalho é a simulação de escoamentos com superfície livre a baixo número de Reynolds, os termos convectivos não são importantes e o tensor tensão não-newtoniano $\mathbf{S}$ é tratado como um termo fonte.

De acordo com o procedimento dos métodos de projeção, utiliza-se a equação (5.10) para calcular a velocidade intermediária, ou seja

$$
\begin{aligned}
\frac{\widetilde{\mathbf{u}}}{\delta t}-\theta_{1} \frac{\gamma}{R e} \nabla^{2} \widetilde{\mathbf{u}} & =\frac{\mathbf{u}^{(n)}}{\delta t}-\nabla \cdot(\mathbf{u u})^{(n)}-\nabla p^{(n)}+\theta_{4} \frac{\gamma}{R e} \nabla^{2} \mathbf{u}^{(n)} \\
& +\beta \nabla \cdot \mathbf{S}^{(n)}+\frac{1}{F r^{2}} \mathbf{g}^{(n)} .
\end{aligned}
$$

Assim como foi realizado na segunda formulação descrita na seção 4.3, pode-se construir a equação para correção da pressão

$$
p^{(n+1)}=p^{(n)}+\psi^{(n+1)}-\gamma \frac{\delta t}{2 R e} \nabla^{2} \psi^{(n+1)},
$$

utilizando as equações (5.10), (5.11) e (2.27).

Após a discretização implícita da equação de quantidade de movimento, o próximo passo na construção do método semi-implícito é tratar a equação da pressão na superfície livre (5.7) implicitamente, enquanto que as condições tangenciais dadas pelas equações (5.8) e (5.9) são tratadas explicitamente. Logo, a equação (5.7), na forma discreta pode ser reescrita como

$$
\begin{aligned}
p^{(n+1)} & =\gamma \frac{2}{R e}\left[\frac{\partial u}{\partial x} n_{x}^{2}+\frac{\partial v}{\partial y} n_{y}^{2}+\frac{\partial w}{\partial z} n_{z}^{2}+\left(\frac{\partial v}{\partial x}+\frac{\partial u}{\partial y}\right) n_{x} n_{y}\right. \\
& \left.+\left(\frac{\partial w}{\partial x}+\frac{\partial u}{\partial z}\right) n_{x} n_{z}+\left(\frac{\partial w}{\partial y}+\frac{\partial v}{\partial z}\right) n_{y} n_{z}\right]^{(n+1)} \\
& +\beta\left[S^{x x} n_{x}^{2}+S^{y y} n_{y}^{2}+S^{z z} n_{z}^{2}+2\left(S^{x y} n_{x} n_{y}+S^{x z} n_{x} n_{z}+S^{y z} n_{y} n_{z}\right)\right]^{(n)}
\end{aligned}
$$

A equação acima acopla velocidade e pressão, e uma estratégia para o desacoplamento destes campos foi essencial para o algoritmo ser eficiente. A construção desta estratégia segue os passos descritos nas seções 4.2 e 4.3 para o caso bidimensional, derivando novas equações para a pressão tentativa $\psi$ na superfície livre. Em trabalhos anteriores, como por exemplo em [155, 161, 145, 143], além da discretização explícita da equação (5.1), a pressão foi calculada explicitamente utilizando a equação (5.7) com $\psi$ sendo definido como zero nas células de superfície livre. A 
seguir, deriva-se as equações para $\psi$ correspondente a cada tipo de aproximação da superfície livre no caso tridimensional.

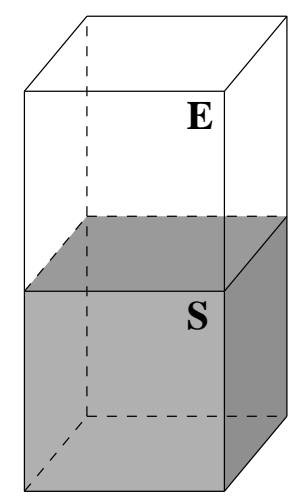

a

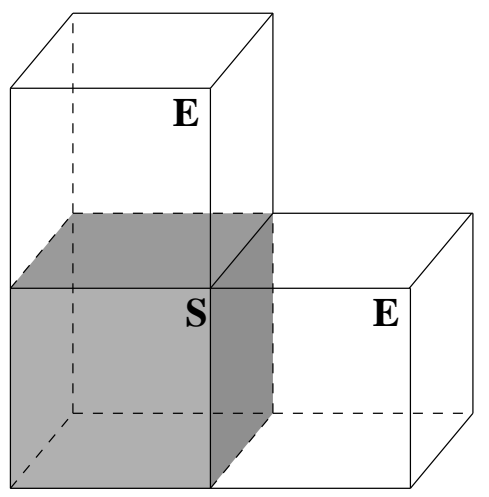

b

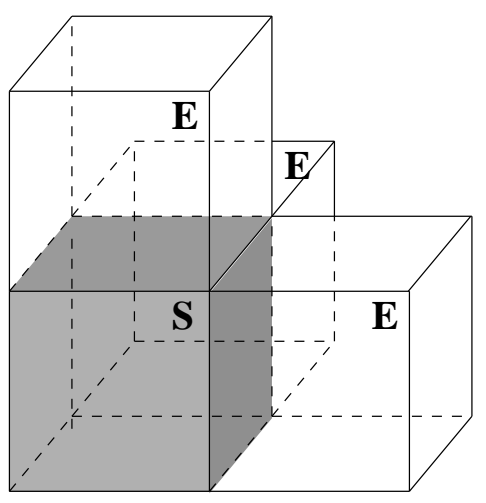

c

Figura 5.1: a) Vetor normal aponta na direção de uma das direções coordenadas, b) vetor normal aponta na direção formando $45^{\circ}$ com dois eixos coordenados adjacentes, e c) vetor normal aponta na direção formando $60^{\circ}$ com três eixos coordenados adjacentes.

i) superfície planar 1D: Para ilustrar como a equação (5.13) deve ser empregada para obter uma equação para a pressão tentativa $\psi$ na superfície livre, considere o caso particular de uma célula $[\mathbf{S}]$ com a face $\left(k+\frac{1}{2}\right)$ em contato com uma célula [E] como apresentado na figura 5.1a. Neste caso, o vetor normal é $\mathbf{n}=(0,0,1)(\mathbf{n}=(0,0,-1)$ resulta na mesma equação) e a equação (5.13) é reduzida a

$$
p^{(n+1)}=\gamma \frac{2}{R e}\left(\frac{\partial w^{(n+1)}}{\partial z}\right)+\beta\left(S^{z z}\right)^{(n)} .
$$

Da equação de conservação de massa (5.2) discretizada no nível de tempo $(n+1)$ obtém-se

$$
\frac{\partial w^{(n+1)}}{\partial z}=-\frac{\partial u^{(n+1)}}{\partial x}-\frac{\partial v^{(n+1)}}{\partial y}
$$

e introduzindo esta equação em (5.14) tem-se

$$
p^{(n+1)}=-\gamma \frac{2}{R e}\left(\frac{\partial u^{(n+1)}}{\partial x}+\frac{\partial v^{(n+1)}}{\partial y}\right)+\beta\left(S^{z z}\right)^{(n)} .
$$


A equação (2.27) discretizada no tempo pode ser reescrita da seguinte forma

$$
\left\{\begin{array}{l}
u^{(n+1)}=\widetilde{u}-\delta t \frac{\partial \psi^{(n+1)}}{\partial x}, \\
v^{(n+1)}=\widetilde{v}-\delta t \frac{\partial \psi^{(n+1)}}{\partial y}, \\
w^{(n+1)}=\widetilde{w}-\delta t \frac{\partial \psi^{(n+1)}}{\partial z},
\end{array}\right.
$$

e introduzindo $u^{(n+1)}$ e $v^{(n+1)}$ na equação (5.16) obtém-se

$$
p^{(n+1)}=\gamma \frac{2}{R e}\left(-\frac{\partial \widetilde{u}}{\partial x}+\delta t \frac{\partial^{2} \psi^{(n+1)}}{\partial x^{2}}-\frac{\partial \widetilde{v}}{\partial y}+\delta t \frac{\partial^{2} \psi^{(n+1)}}{\partial y^{2}}\right)+\beta\left(S^{z z}\right)^{(n)}
$$

Finalmente, substituindo a equação (5.12) em (5.18), e re-agrupando os termos, obtém-se a seguinte equação para $\psi^{(n+1)}$ na superfície livre

$$
\begin{aligned}
\psi^{(n+1)} & -\gamma \frac{\delta t}{2 R e} \nabla^{2} \psi^{(n+1)}-\gamma \frac{2 \delta t}{R e}\left(\frac{\partial^{2} \psi^{(n+1)}}{\partial x^{2}}+\frac{\partial^{2} \psi^{(n+1)}}{\partial y^{2}}\right) \\
& =-\gamma \frac{2}{R e}\left(\frac{\partial \widetilde{u}}{\partial x}+\frac{\partial \widetilde{v}}{\partial y}\right)-p^{(n)}+\beta\left(S^{z z}\right)^{(n)}
\end{aligned}
$$

Os outros casos para a superfície planar $1 \mathrm{D} \operatorname{com} \mathbf{n}=(0, \pm 1,0)$ e $\mathbf{n}=( \pm 1,0,0)$ são tratados de forma semelhante.

ii) superfície planar 2D: Considere a célula $[\mathrm{S}]$ como apresentado na figura 5.1b. Neste caso, assume-se que o vetor normal é dado por $\mathbf{n}=\left(\frac{\sqrt{2}}{2}, 0, \frac{\sqrt{2}}{2}\right)$ e a equação (5.13) é descrita como

$$
\begin{aligned}
p^{(n+1)} & =\frac{\gamma}{R e}\left(\frac{\partial u^{(n+1)}}{\partial x}+\frac{\partial w^{(n+1)}}{\partial z}+\frac{\partial u^{(n+1)}}{\partial z}+\frac{\partial w^{(n+1)}}{\partial x}\right) \\
& +\beta\left[\frac{1}{2} S^{x x}+\frac{1}{2} S^{z z}+S^{x z}\right]^{(n)}
\end{aligned}
$$

Impondo a conservação de massa, obtém-se

$$
\begin{aligned}
p^{(n+1)} & =\frac{\gamma}{R e}\left(-\frac{\partial v^{(n+1)}}{\partial y}+\frac{\partial u^{(n+1)}}{\partial z}+\frac{\partial w^{(n+1)}}{\partial x}\right) \\
& +\beta\left[\frac{1}{2} S^{x x}+\frac{1}{2} S^{z z}+S^{x z}\right]^{(n)} .
\end{aligned}
$$


Agora, substituindo $u^{(n+1)}, v^{(n+1)}$ e $w^{(n+1)}$ da equação (5.17) em (5.21) tem-se

$$
\begin{aligned}
p^{(n+1)} & =\frac{\gamma}{R e}\left(-\frac{\partial \widetilde{v}}{\partial y}+\delta t \frac{\partial^{2} \psi^{(n+1)}}{\partial y^{2}}+\frac{\partial \widetilde{u}}{\partial z}-\delta t \frac{\partial^{2} \psi^{(n+1)}}{\partial x \partial z}\right. \\
& \left.+\frac{\partial \widetilde{w}}{\partial x}-\delta t \frac{\partial^{2} \psi^{(n+1)}}{\partial x \partial z}\right)+\beta\left[\frac{1}{2} S^{x x}+\frac{1}{2} S^{z z}+S^{x z}\right]^{(n)}
\end{aligned}
$$

A equação para $\psi^{(n+1)}$ é obtida quando substitui-se (5.12) na equação (5.22), e após o reagrupamento dos termos constrói-se

$$
\begin{aligned}
\psi^{(n+1)} & -\gamma \frac{\delta t}{2 R e} \nabla^{2} \psi^{(n+1)}-\gamma \frac{\delta t}{R e}\left(\frac{\partial^{2} \psi^{(n+1)}}{\partial y^{2}}-2 \frac{\partial^{2} \psi^{(n+1)}}{\partial x \partial z}\right) \\
& =-\frac{\gamma}{R e}\left(\frac{\partial \widetilde{v}}{\partial y}-\frac{\partial \widetilde{u}}{\partial z}-\frac{\partial \widetilde{w}}{\partial x}\right)-p^{(n)}+\beta\left[\frac{1}{2} S^{x x}+\frac{1}{2} S^{z z}+S^{x z}\right]^{(n)}
\end{aligned}
$$

A equação (5.23) é utilizada para uma célula $[\mathbf{S}]$ com as faces $\left(i+\frac{1}{2}\right)$ e $\left(k+\frac{1}{2}\right)$ em contato com células $[\mathbf{E}]$. As equações correspondentes para as outras configurações de células $[\mathbf{S}]$ com duas faces adjacentes com células $[\mathbf{E}]$ são obtidas de forma semelhante.

iii) superfície planar 3D: Para este tipo de configuração são consideradas 8 possibilidades. Como ilustração, apresenta-se a equação para uma célula $[\mathbf{S}]$ com as faces $\left(i+\frac{1}{2}\right),\left(j+\frac{1}{2}\right)$ e $\left(k+\frac{1}{2}\right)$ em contato com faces de células [E] (veja na figura 5.1c). Neste caso, assume-se $\mathbf{n}=\left(\frac{\sqrt{3}}{3}, \frac{\sqrt{3}}{3}, \frac{\sqrt{3}}{3}\right)$ e a equação (5.13) toma a forma

$$
\begin{aligned}
p^{(n+1)} & =\gamma \frac{2}{3 R e}\left(\frac{\partial u^{(n+1)}}{\partial y}+\frac{\partial v^{(n+1)}}{\partial x}+\frac{\partial u^{(n+1)}}{\partial z}+\frac{\partial w^{(n+1)}}{\partial x}+\frac{\partial v^{(n+1)}}{\partial z}\right. \\
& \left.+\frac{\partial w^{(n+1)}}{\partial y}\right)+\beta \frac{1}{3}\left[S^{x x}+S^{y y}+S^{z z}+2\left(S^{x y}+S^{x z}+S^{y z}\right)\right]^{(n)}
\end{aligned}
$$

e introduzindo $u^{(n+1)}, v^{(n+1)}$ e $w^{(n+1)}$ da equação (5.17), obtém-se

$$
\begin{aligned}
p^{(n+1)} & =\gamma \frac{2}{3 R e}\left(\frac{\partial \widetilde{u}}{\partial y}+\frac{\partial \widetilde{u}}{\partial z}+\frac{\partial \widetilde{v}}{\partial x}+\frac{\partial \widetilde{v}}{\partial z}+\frac{\partial \widetilde{w}}{\partial x}+\frac{\partial \widetilde{w}}{\partial y}\right. \\
& \left.-2 \delta t\left[\frac{\partial^{2} \psi^{(n+1)}}{\partial y \partial x}+\frac{\partial^{2} \psi^{(n+1)}}{\partial z \partial x}+\frac{\partial^{2} \psi^{(n+1)}}{\partial z \partial y}\right]\right) \\
& +\beta \frac{1}{3}\left[S^{x x}+S^{y y}+S^{z z}+2\left(S^{x y}+S^{x z}+S^{y z}\right)\right]^{(n)}
\end{aligned}
$$


A equação final para $\psi^{(n+1)}$ é obtida introduzindo (5.12) na equação (5.25) e, após algumas simplificações, resume-se em

$$
\begin{aligned}
\psi^{(n+1)} & -\gamma \frac{\delta t}{2 R e} \nabla^{2} \psi^{(n+1)}+\gamma \frac{4 \delta t}{3 R e}\left[\frac{\partial^{2} \psi^{(n+1)}}{\partial y \partial x}+\frac{\partial^{2} \psi^{(n+1)}}{\partial z \partial x}+\frac{\partial^{2} \psi^{(n+1)}}{\partial z \partial y}\right] \\
& =\gamma \frac{2}{3 R e}\left(\frac{\partial \widetilde{u}}{\partial y}+\frac{\partial \widetilde{u}}{\partial z}+\frac{\partial \widetilde{v}}{\partial x}+\frac{\partial \widetilde{v}}{\partial z}+\frac{\partial \widetilde{w}}{\partial x}+\frac{\partial \widetilde{w}}{\partial y}\right) \\
& +\beta \frac{1}{3}\left[S^{x x}+S^{y y}+S^{z z}+2\left(S^{x y}+S^{x z}+S^{y z}\right)\right]^{(n)}-p^{(n)}
\end{aligned}
$$

As equações correspondente às demais configurações de superfície planar 3D são construídas da mesma forma.

Nota-se que na resolução destas equações para células [S], os valores de $\psi$ em células [E] são definidos como zero.

\subsubsection{Algoritmo}

Supõe-se que no tempo $t_{0}$, o campo de velocidade $\mathbf{u}$ é conhecido e as condições de contorno descritas no capítulo 2 são dadas. O campo de velocidade atualizado $\mathbf{u}$, a pressão $p$ e o tensor tensão não-newtoniano $\mathbf{S}$ no tempo $t=t_{0}+\delta t$ são calculados com os seguinte passos:

- Passo 1: Calcular o campo de velocidade intermediário $\widetilde{\mathbf{u}}$ da equação (5.11). Neste passo as duas formulações semi-implícitas descritas nas seções 4.2 e 4.3 foram estendidas:

- Para escoamentos de fluido do tipo Maxwell utiliza-se o método de Euler implícito, ou seja, $\gamma=\beta=1, \theta_{1}=1$ e $\theta_{4}=0$ na equação (5.11).

- Para escoamentos de fluidos newtoniano e do tipo Oldroyd-B utiliza-se o método de Crank-Nicolson usando $\theta_{1}=\theta_{4}=0.5$ na equação (5.11). Além disso, no caso de fluido newtoniano assume-se $\gamma=1$ e $\beta=0$, enquanto que para fluido do tipo Oldroyd-B tem-se $\gamma=\frac{\lambda_{2}}{\lambda_{1}}$ e $\beta=1$ em (5.11).

A imposição das condições de contorno para $\widetilde{u}$ na equação (5.11) segue a mesma estratégia do caso bidimensional descrita anteriormente nos algoritmos 4.2.1 e 4.3.1.

- Passo 2: Resolver a equação de Poisson

$$
\nabla^{2} \psi^{(n+1)}=\frac{1}{\delta t} \nabla \cdot \widetilde{\mathbf{u}}
$$


junto com as equações derivadas para a pressão tentativa $\psi^{(n+1)}$ na superfície livre, descritas no início desta seção. Neste passo, tem-se:

- Para escoamentos de fluido do tipo Maxwell deve ser imposto $\gamma=\beta=1$ nas equações (5.19), (5.23) e (5.26).

- Para escoamentos de fluidos newtonianos deve ser imposta $\gamma=1$ e $\beta=0$ nas equações (5.19), (5.23) e (5.26), enquanto para fluido do tipo Oldroyd-B tem-se $\gamma=\frac{\lambda_{2}}{\lambda_{1}}$ e $\beta=1$.

As condições de contorno para este conjunto de equações são $\psi^{(n+1)}=0$ nos ejetores e condições de Neumann homogênea (2.29) nas paredes rígidas e nos injetores. O sistema linear resultante para $\psi^{(n+1)}$ é esparso e não-simétrico, e da mesma forma que no caso bidimensional, emprega-se o método dos gradientes bi-conjugados com pré-condicionamento para resolver este sistema linear.

- Passo 3: Atualizar o campo de velocidade final pela equação (5.17).

- Passo 4: Atualizar o campo de pressão final pela equação (5.12).

- Passo 5: Calcular o tensor tensão não-newtoniano S. Neste passo considera-se:

- Para fluido do tipo Maxwell

$$
\begin{aligned}
\frac{\partial \mathbf{S}}{\partial t} & =-\nabla \cdot\left(\mathbf{u}^{(n+1)} \mathbf{S}\right)+\left(\nabla \mathbf{u}^{(n+1)}\right)^{T} \mathbf{S}+\mathbf{S}\left(\nabla \mathbf{u}^{(n+1)}\right)-\frac{1}{W e} \mathbf{S} \\
& -\frac{2}{R e}\left[\nabla \cdot\left(\mathbf{u}^{(n+1)} \mathbf{D}^{(n+1)}\right)-\left(\nabla \mathbf{u}^{(n+1)}\right)^{T} \mathbf{D}^{(n+1)}-\mathbf{D}^{(n+1)}\left(\nabla \mathbf{u}^{(n+1)}\right)\right],
\end{aligned}
$$

onde $\mathbf{D}^{(n+1)}=\frac{1}{2}\left[\nabla \mathbf{u}^{(n+1)}+\left(\nabla \mathbf{u}^{(n+1)}\right)^{T}\right]$.

- Para fluido do tipo Oldroyd-B tem-se

$$
\begin{aligned}
\frac{\partial \mathbf{S}}{\partial t} & =-\nabla \cdot\left(\mathbf{u}^{(n+1)} \mathbf{S}\right)+\left(\nabla \mathbf{u}^{(n+1)}\right)^{\mathrm{T}} \mathbf{S}+\mathbf{S}\left(\nabla \mathbf{u}^{(n+1)}\right)-\frac{1}{W e} \mathbf{S} \\
& +\frac{1}{W e}\left[\frac{1}{R e}\left(1-\frac{\lambda_{2}}{\lambda_{1}}\right)\left(\left(\nabla \mathbf{u}^{(n+1)}\right)+\left(\nabla \mathbf{u}^{(n+1)}\right)^{\mathrm{T}}\right)\right] .
\end{aligned}
$$

As equações (5.27) e (5.28) são calculadas explicitamente pelo método de Euler explícito, e os detalhes de implementação podem ser encontrados em Silva e Tomé [143] e Tomé et al. [165] para o modelo de Maxwell e em Tomé et al. [157] para o modelo Oldroyd-B. 
- Passo 6: Determinar as novas posições das partículas marcadoras virtuais resolvendo a equação

$$
\dot{\mathbf{x}}=\mathbf{u}^{(n+1)} \text {. }
$$

Neste passo, assim como no algoritmo das formulações bidimensionais, utiliza-se o esquema de Euler explícito para resolver as equações acima. Detalhes da implementação deste esquema no sistema FREEFLOW foram apresentados em Castelo et al. [15].

\subsection{Verificação}

Nesta seção, apresenta-se testes tridimensionais para a verificação dos métodos de projeção com superfície livre descritos acima. Novamente, utilizou-se o sistema FREEFLOW e um Sun Fire AMD Athlon(tm) 64/Opteron(tm), 2193 MHz, 24Gb de memória RAM, 4 processadores Dual core, com o sistema Solaris. Em todas as simulações utilizou-se uma malha estruturada uniforme, e para as soluções dos sistemas lineares envolvidos utilizou-se a tolerância $\epsilon=1.0 \times$ $10^{-10}$.

Como a formulação para o fluido do tipo Maxwell utiliza o esquema de Euler implícito combinada com as técnicas implícitas na superfície livre (veja a seção 5.2), utiliza-se a mesma notação do caso bidimensional, SI-EI/CCE. Para o fluido Oldroyd-B aplica-se o método de Crank-Nicolson com técnicas implícitas nas condições de contorno (veja na seção 5.2), então, seguindo também a notação do caso bidimensional, utiliza-se SI-CN/CCI1.

As verificações são realizadas para escoamentos em um tubo, como ilustrado na figura 5.2. Neste caso, impõem-se um perfil parabólico na entrada do tubo dado por

$$
w(x, y)=\frac{2 U\left[R^{2}-x^{2}-y^{2}\right]}{R^{2}}, \quad u=v=0
$$

onde $R$ é o raio interno do tubo e $U$ a velocidade de escala.

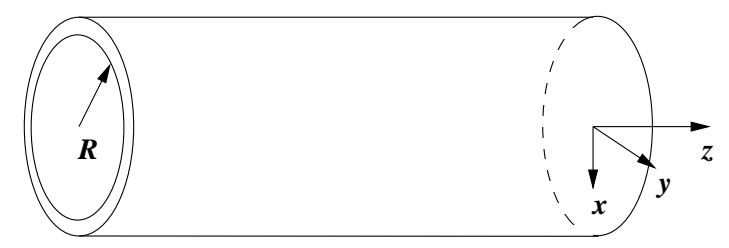

Figura 5.2: Domínio para a simulação do escoamento em um tubo. 


\subsubsection{Fluido do tipo Maxwell}

Introduzindo o perfil (5.29) na equação (5.3), e assumindo o escoamento apenas na direção $z$, pode-se obter facilmente as seguintes relações

$$
S^{x x}(x, y)=S^{y y}(x, y)=S^{x y}(x, y)=S^{x z}(x, y)=S^{y z}(x, y)=0
$$

$\mathrm{e}$

$$
S^{z z}(x, y)=2 \frac{W e}{R e}\left[\left(\frac{\partial w}{\partial x}\right)^{2}+\left(\frac{\partial w}{\partial y}\right)^{2}\right]
$$

Para verificar a formulação SI-EI/CCE foi simulado o escoamento em um tubo com raio $R$ e comprimento $10 R$. As soluções numéricas foram comparadas com as respectivas soluções exatas dadas pelas equações (5.29) e (5.31). As condições de contorno para a velocidade são impostas de acordo com (2.4) nas paredes rígidas do tubo, enquanto que no ejetor assume-se condições de Neumann homogênea. A descrição das condições de contorno para o tensor não-newtoniano $\mathbf{S}$ pode ser encontrada em Tomé et al. [162, 165] e em Silva e Tomé [143]. Na entrada do tubo, assume-se as soluções exatas dadas pelas equações (5.29), (5.30) e (5.31).

Os parâmetros de entrada utilizados foram: $R=0.005 \mathrm{~m}, U=1 \mathrm{~m} \mathrm{~s}^{-1}, \mu=10 \mathrm{Pas}, \rho=$ $1000 \mathrm{~kg} \mathrm{~m}^{-3}$ e $\lambda_{1}=0.002 \mathrm{~s}$, tal que $R e=\frac{\rho U L}{\mu}=0.5$ e $W e=\frac{\lambda_{1} U}{R}=0.4$. Neste caso a gravidade não foi imposta. Para analisar a convergência do método utilizou-se o refinamento de malha empregando-se as seguintes malhas uniformes: M1 - $16 \times 16 \times 80$ células $\left(\delta x_{M 1}=0.1250 \mathrm{~m}\right)$, M2 - $20 \times 20 \times 100$ células $\left(\delta x_{M 2}=0.1 \mathrm{~m}\right)$ e M3 $-24 \times 24 \times 120$ células $\left(\delta x_{M 3}=0.083 \mathrm{~m}\right)$.

A simulação inicia-se com o tubo vazio e o fluido é injetado na entrada com a velocidade dada por (5.29) até atingir o ejetor, entrando em estado estacionário. Em estado estacionário, a velocidade e os tensores, em qualquer seção do tubo, devem apresentar os mesmos valores que os impostos no injetor.

As soluções numéricas obtidas para a velocidade $w$ e para a componente do tensor nãonewtoniano $S^{z z}$ em uma seção transversal localizada no meio do tubo $(z / R=5)$ são apresentadas na figura 5.3. Nota-se que há uma boa concordância entre as soluções numéricas e as exatas. A tabela 5.1 apresenta os erros relativos calculados na norma $l_{2}$. Os erros decrescem quando a malha é refinada evidenciando a convergência do método utilizado. 
Tabela 5.1: Erros obtidos nas malhas M1, M2 e M3 para a formulação SI-EI/CCE no escoamento em um tubo para o fluido do tipo Maxwell.

\begin{tabular}{|c|c|c|}
\hline Malha & $\operatorname{Er}(w)$ & $\operatorname{Er}\left(S^{z z}\right)$ \\
\hline M1 & $2.6494 \times 10^{-3}$ & $1.0949 \times 10^{-2}$ \\
M2 & $1.5804 \times 10^{-3}$ & $6.8301 \times 10^{-3}$ \\
M3 & $1.0563 \times 10^{-3}$ & $5.0099 \times 10^{-3}$ \\
\hline
\end{tabular}
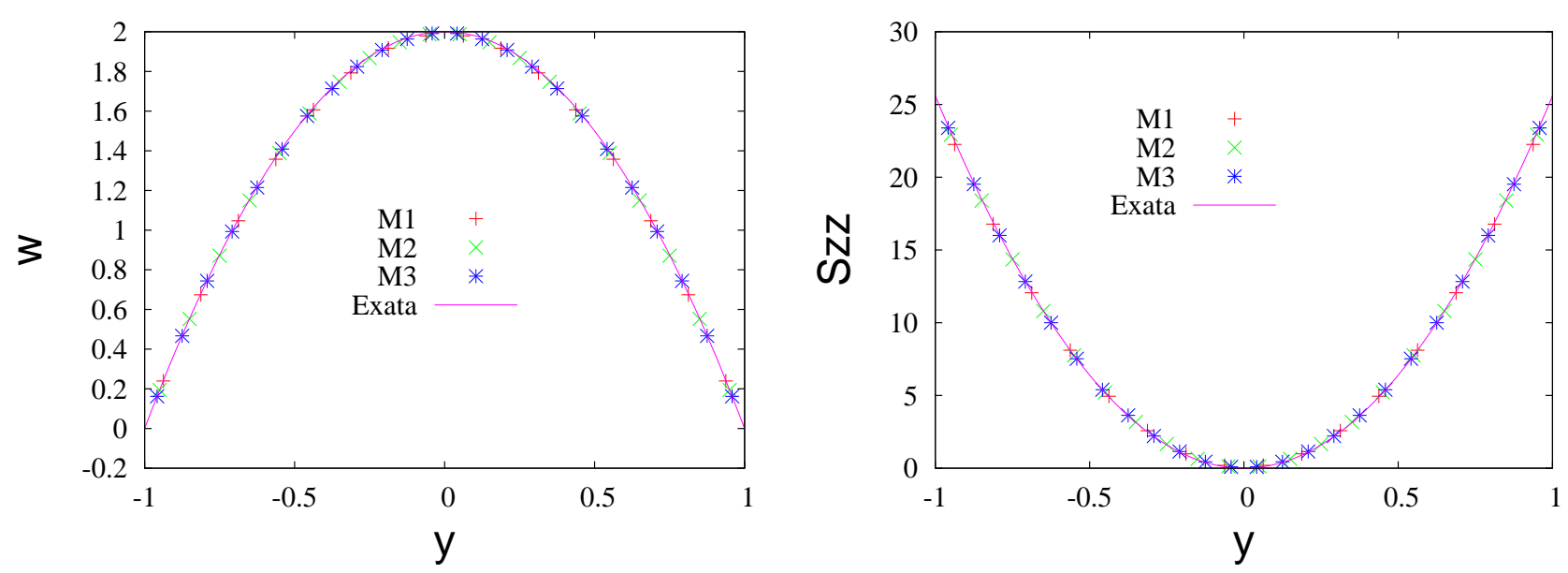

Figura 5.3: Soluções numéricas obtida nas malhas M1, M2 e M3. As soluções numéricas e exatas foram calculadas em $x=0,-1 \leq y / R \leq 1$ e $z / R=5$.

\subsubsection{Fluido do tipo Oldroyd-B}

Considera-se um tubo de raio $R$ e comprimento $5 R$ (ver figura 5.2) e impõem-se um perfil parabólico na entrada do tubo dado por (5.29). Neste caso, pode-se mostrar que a solução analítica para as componentes do tensor tensão não-newtoniano $\mathbf{S}$ são dadas por

$$
\left\{\begin{array}{l}
S^{x y}=0, \quad S^{x z}=\frac{1}{R e}\left(1-\frac{\lambda_{2}}{\lambda_{1}}\right) \frac{\partial w}{\partial x}, \quad S^{y z}=\frac{1}{R e}\left(1-\frac{\lambda_{2}}{\lambda_{1}}\right) \frac{\partial w}{\partial y} \\
S^{x x}=S^{y y}=0, \quad S^{z z}=2 \frac{W e}{R e}\left(1-\frac{\lambda_{2}}{\lambda_{1}}\right)\left[\left(\frac{\partial w}{\partial x}\right)^{2}+\left(\frac{\partial w}{\partial y}\right)^{2}\right]
\end{array}\right.
$$

Os dados acima foram programados no sistema FREEFLOW para a verificação da formulação SI-CN/CCI1 (veja na seção 5.2). Novamente, inicialmente o tubo está vazio e injeta-se fluido na entrada, até que o escoamento atinja o estado estacionário.

Os parâmetros de entrada utilizados foram: $R=1 \mathrm{~m}$, sem gravidade, $\mu=10$ Pa.s, $\rho=$ $1 \mathrm{~kg} \mathrm{~m}^{-3}, \lambda_{1}=1 \mathrm{~s}$ e $\lambda_{2}=0.5 \mathrm{~s}$. Os parâmetros de escala foram $L=R, U=1 \mathrm{~ms}^{-1}$. Logo, 
$R e=0.1$ e $W e=1$. Neste caso, as malhas utilizadas foram: M0 - $12 \times 12 \times 30$ células $\left(\delta x_{M 0}=0.1667 m\right), \mathrm{M} 1-16 \times 16 \times 40$ células $\left(\delta x_{M 1}=0.1250 \mathrm{~m}\right), \mathrm{M} 2-22 \times 22 \times 55$ células $\left(\delta x_{M 2}=0.0909 \mathrm{~m}\right)$ e M3 $-28 \times 28 \times 70$ células $\left(\delta x_{M 3}=0.071428 \mathrm{~m}\right)$. Os escoamentos foram integrados de $t=0 \mathrm{~s}$ até $t=50 \mathrm{~s}$.

Neste problema, utilizou-se $\delta t=1.15742 \times 10^{-3} s$ para a malha M0, $\delta t=6.51000 \times 10^{-4} s$ para a malha M1, $\delta t=6.68800 \times 10^{-5} s$ para a malha M2 e $\delta t=4.25170 \times 10^{-5} s$ para a malha M3. Com o objetivo de verificar a eficiência do esquema implícito, foi simulado o mesmo problema para a malha M0 utilizando o método explícito GENSMAC de Tomé et al. [157]. Nesta simulação, o tamanho do passo temporal utilizado para manter a estabilidade do método explícito foi $\delta t=4.62963 \times 10^{-5} \mathrm{~s}$. Para comparar a precisão de ambas as técnicas, novamente considerou-se a secção transversal do tubo, agora em $z=2.5 R$ e calculou-se o erro relativo na norma $l_{2}$.

A tabela 5.2 apresenta o tempo de processamento para os métodos explícito e implícito, além dos respectivos erros na norma $l_{2}$ utilizando a malha M0. Da tabela 5.2 é possível verificar que a técnica implícita utilizou um $\delta t 25$ vezes maior que o passo temporal utilizado pelo esquema explícito, e consequentemente a redução do tempo de processamento foi muito significativa. Além disso, observa-se que os erros obtidos pela técnica implícita foram da mesma ordem que os erros do método explícito de Tomé et al. [157]. Os erros $\operatorname{Er}\left(S^{x z}\right)$ e $\operatorname{Er}\left(S^{y z}\right)$ foram praticamente os mesmos para ambas as técnicas, e esta é a razão para não serem apresentados.

Tabela 5.2: Comparação da performance dos esquemas explícito e implícito na malha M0.

\begin{tabular}{|c|c|c|c|c|}
\hline Método & $\operatorname{Er}(w)$ & $\operatorname{Er}\left(S^{z z}\right)$ & $\delta t(s)$ & $\begin{array}{c}\text { Tempo de processamento } \\
\text { (minutos : segundos) }\end{array}$ \\
\hline GENSMAC [157] & $5.219 \times 10^{-3}$ & $2.058 \times 10^{-2}$ & $4.629 \times 10^{-5}$ & $9358: 11$ \\
SI-CN/CCI1 & $5.219 \times 10^{-3}$ & $2.058 \times 10^{-2}$ & $1.157 \times 10^{-3}$ & $294: 27$ \\
\hline
\end{tabular}

Para analisar a convergência do método SI-CN/CCI1 apresenta-se na tabela 5.3, os erros obtidos no cálculo da velocidade $w$ utilizando três malhas. Adicionalmente, a tabela 5.3 também apresenta os erros no cálculo do tensor não-newtoniano S. Pode-se observar na tabela 5.3 que os erros decrescem com o refinamento da malha, demonstrando a convergência da técnica implícita para escoamentos a baixo número de Reynolds. 
Tabela 5.3: Erros nas malhas M1, M2 e M3 para o escoamento em um tubo com o método SI-CN/CCI1.

\begin{tabular}{|c|c|c|c|c|}
\hline Malha & $\operatorname{Er}(w)$ & $\operatorname{Er}\left(S^{z z}\right)$ & $\operatorname{Er}\left(S^{x z}\right)$ & $\operatorname{Er}\left(S^{y z}\right)$ \\
\hline M1 & $2.6455 \times 10^{-3}$ & $9.4005 \times 10^{-3}$ & $4.8908 \times 10^{-3}$ & $4.8692 \times 10^{-3}$ \\
M2 & $1.3845 \times 10^{-3}$ & $6.9864 \times 10^{-3}$ & $3.8249 \times 10^{-3}$ & $3.7656 \times 10^{-3}$ \\
M3 & $8.5889 \times 10^{-4}$ & $3.9572 \times 10^{-3}$ & $2.0880 \times 10^{-3}$ & $2.0223 \times 10^{-3}$ \\
\hline
\end{tabular}

Neste caso a ordem de precisão espacial $N_{i}$ do método SI-CN/CCI1 pode ser calculada por (4.21) com $\Gamma=w$ e com os erros na norma $l_{2}$. Desta forma tem-se $N_{1}=2.03$ e $N_{2}=1.98$ resultando em $N_{m}=\frac{N_{1}+N_{2}}{2} \simeq 2.00$.

\subsection{Aplicações}

Para demonstrar que as formulações apresentadas na seção 5.2 podem simular escoamentos com superfície livre a baixo número de Reynolds, dois importantes problemas foram simulados: o jato oscilante e o inchamento do extrudado.

\subsubsection{Simulação numérica do jato oscilante}

O fenômeno do jato oscilante (jet buckling phenomenon) acontece quando um fluido viscoso, injetado de uma certa altura, atinge uma parede rígida e desacelera, formando um acumulo de fluido. Este problema tem atraido a atenção de muitos investigadores, e resultados experimentais e numéricos, mostrando detalhes deste fenômeno para o caso tridimensional newtoniano, podem ser encontrados nos trabalhos de Cruickshank e Munson [31], Tomé et al. [155], Ribe [136, 137, 138], Nobrega et al. [112], entre outros.

Como uma verificação adicional para a formulação SI-CN/CCI1, simulou-se o fenômeno do jato oscilante de um fluido viscoso. Considerou-se um jato axisimétrico de um fluido newtoniano a baixo número de Reynolds escoando dentro de uma caixa inicialmente vazia. O efeito das oscilações (também conhecido como flambagem) ocorre devido à alta viscosidade quando o fluido alcança o fundo da caixa. Este problema foi simulado utilizando os métodos explícito GENSMAC [155] e SI-CN/CCI1 apresentado na seção 5.2.

Os dados do modelo utilizados no sistema FREEFLOW para esta simulação foram: $\mu=$ 3.5Pa.s, $\rho=1380 \mathrm{~kg} \mathrm{~m}^{-3}$, diâmetro do injetor $(D)$ igual a $6 \mathrm{~mm}$ e altura do injetor até o 
fundo da caixa $(H)$ foi $11 \mathrm{~cm}$. Uma caixa de dimensões $4 \mathrm{~cm} \times 4 \mathrm{~cm} \times 2 \mathrm{~cm}$ foi utilizada. No injetor, impõem-se um perfil parabólico (Poiseuille profile) dado pela equação (5.29) com $U=0.116 \mathrm{~m} \mathrm{~s}^{-1}$. Neste caso assume-se que a gravidade está agindo com $g=9.81 \mathrm{~m} \mathrm{~s}^{-2}$. Uma malha de $60 \times 60 \times 110$ células $(\delta x=\delta y=\delta z=1 \mathrm{~mm})$ foi utilizada. O tamanho do passo temporal do método explícito foi calculado de acordo com a restrição dada por Tomé et al. [157]. Neste caso, o esquema implícito utilizou um $\delta t 100$ vezes maior que o esquema explícito.

De acordo com os parâmetros adimensionais dados acima, nesta simulação tem-se $R e=$ 0.274 e $F r=0.478$. Cruickshank e Munson [31] analisaram uma série de experimentos para o fenômeno do jato oscilante, e concluíram que um jato 3D apresentará flambagem se as seguintes condições forem satisfeitas: $R e<1.2$ e $H / D>7.2$. Nesta simulação, tem-se $R e=0.274<1.2$ e $H / D=18.33>7.2$, e portanto, de acordo com os autores em [31], a expectativa é observar o efeito do jato oscilante.

O sistema FREEFLOW simulou este problema utilizando as formulações GENSMAC e SI-CN $/$ CCI1 até $t=1.20$ s e os resultados são apresentados na figura 5.4. Nota-se na figura 5.4 que ambas estratégias apresentaram o fenômeno do jato oscilante. Além disso, observa-se que os resultados obtidos utilizando a formulação SI-CN/CCI1 são semelhantes aos resultados do esquema explícito. Portanto, estes resultados providenciam uma verificação qualitativa da implementação da formulação SI-CN/CCI1, no sistema FREEFLOW.

Para verificar a eficiência e robustez da metodologia descrita, o sistema FREEFLOW simulou este problema até o tempo 2.80s (veja figura 5.5). É possível verificar na figura 5.5 que a partir do tempo $t=1.60$ s o fluido atingiu as paredes laterais da caixa e continua a preencher o recipiente.

Após esta verificação, utilizou-se a formulação SI-CN/CCI1 para o fenômeno do jato newtoniano oscilante para $R e<<1$.

Os dados do modelo implementados no sistema FREEFLOW para esta simulação foram: $\mu=4,20,40,200$ Pa.s, $\rho=1000 \mathrm{~kg} \mathrm{~m}^{-3}$ resultando nos valores de $R e=0.5,0.1,0.05,0.01$, respectivamente. O diâmetro do injetor (D) foi $4 \mathrm{~mm}$ e altura do injetor até o fundo da caixa (H) igual a $6 \mathrm{~cm}$. Uma caixa de dimensões $6 \mathrm{~cm} \times 6 \mathrm{~cm} \times 3 \mathrm{~cm}$ foi utilizada. No injetor, impõem-se um perfil parabólico (Poiseuille profile) dado pela equação (5.29) com $U=0.5 \mathrm{~m} \mathrm{~s}^{-1}$. Assumese que a gravidade está agindo com $g=9.81 \mathrm{~m} \mathrm{~s}^{-2}$. Uma malha de $80 \times 80 \times 120$ células $(\delta x=\delta y=\delta z=1 \mathrm{~mm})$ foi utilizada.

Nestas simulações $H / D=15>7.2$ e $R e<1$, portanto espera-se o efeito da flambagem em todos as simulações. Os resultados das quatro simulações são apresentados nas figuras 5.6- 


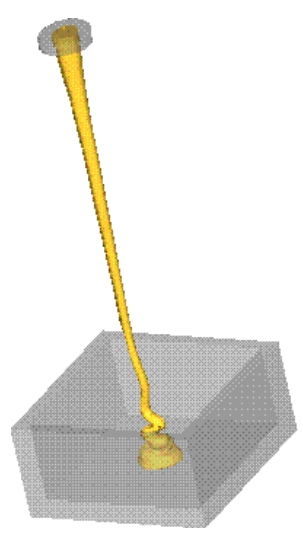

$t=0.32 \mathrm{~s}$

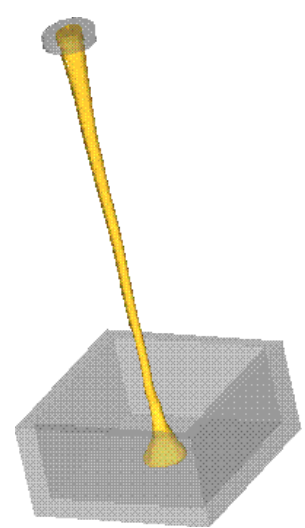

$t=0.32 \mathrm{~s}$

Formulação GENSMAC

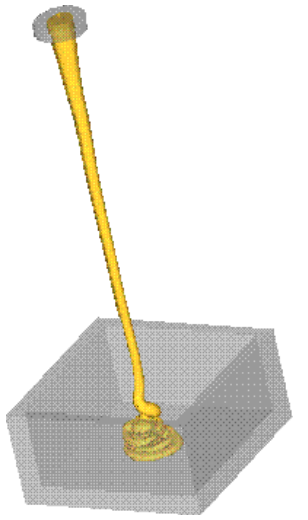

$t=0.40 \mathrm{~s}$

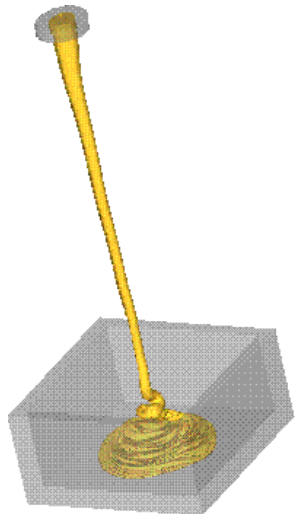

$t=0.80 \mathrm{~s}$

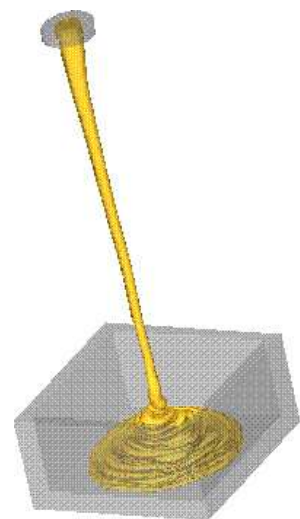

$t=1.20 \mathrm{~s}$

\section{Formulação SI-CN/CCI1}

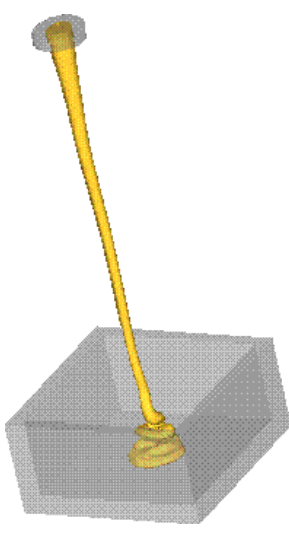

$t=0.40 s$

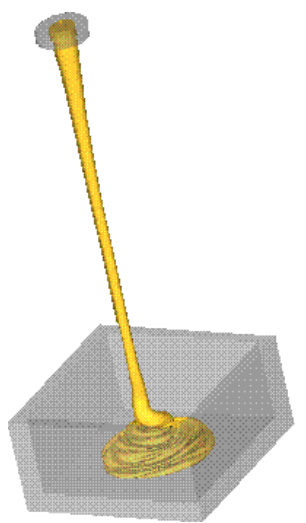

$t=0.80 \mathrm{~s}$

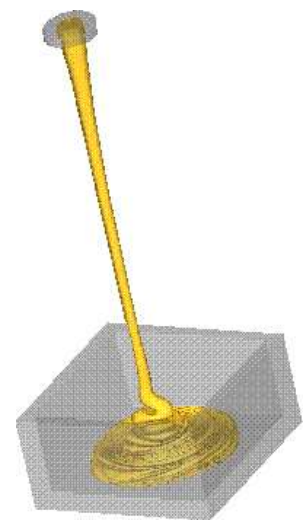

$t=1.20 \mathrm{~s}$

Figura 5.4: Simulação numérica do jato oscilante utilizando as formulações explícita (GENSMAC [155]) e implícita (SI-CN/CCI1, descrita na seção 5.2). Visualização do escoamento em tempos selecionados. ( $R e=0.274$ e $F r=0.478$, fluido newtoniano).

5.9. Os resultados para $R e=0.5$ (ver figura 5.6) e $R e=0.1$ (ver figura 5.7) mostram que os jatos iniciaram o efeito da flambagem anteriormente aos jatos com $R e=0.05$ (ver figura 5.8) e $R e=0.01$ (ver figura 5.9). Nota-se, em todas as simulações, o efeito do enrolamento do jato. Detalhes sobre este efeito podem ser encontrados em [31, 136, 137, 155]. Além disso, quando o número de Reynolds decresce observa-se que os jatos tornam-se mais espessos e dobram de forma caótica (ver figuras 5.8 e 5.9). Este efeito de enrolamento tem sido investigado experimentalmente por Ribe et al.[137, 138], e é conhecido como coiling.

A formulação implícita utilizando o método de Euler implícito, descrita na seção 5.2 e denotada neste capítulo por SI-EI/CCE, também foi verificada para a simulação numérica do 


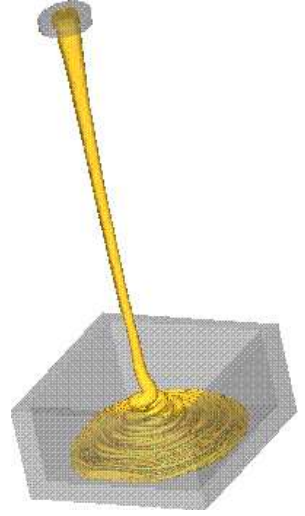

$t=1.60 \mathrm{~s}$
Formulação SI-CN/CCI1

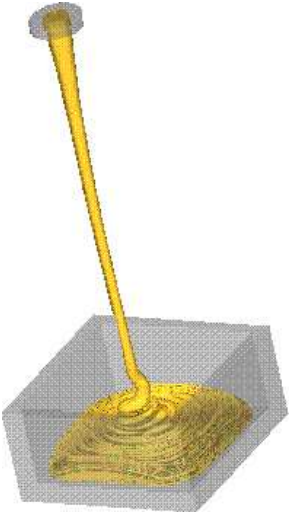

$t=2.0 \mathrm{~s}$

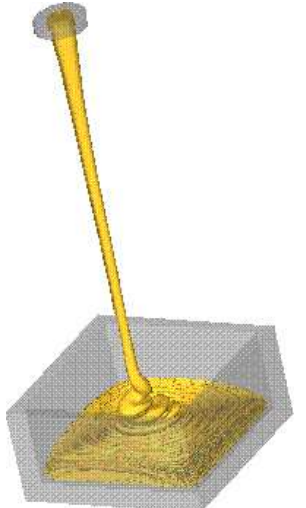

$t=2.40 \mathrm{~s}$

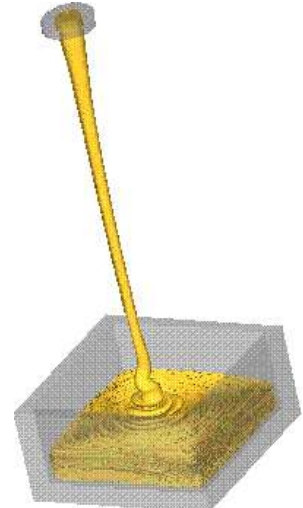

$t=2.80 \mathrm{~s}$

Figura 5.5: Simulação numérica do jato oscilante utilizando a formulação implícita (SI$\mathrm{CN} / \mathrm{CCI} 1$, descrita na seção 5.2). Visualização do escoamento em tempos selecionados. $(R e=0.274$ e $F r=0.478$, fluido newtoniano).

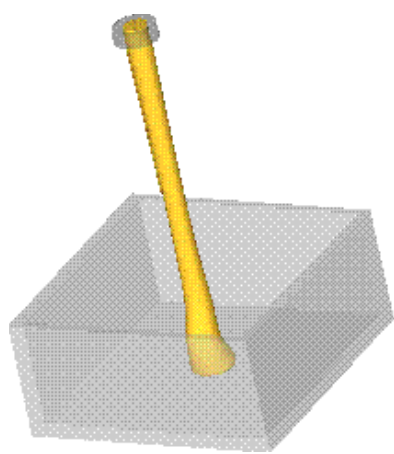

$t=0.14 s$

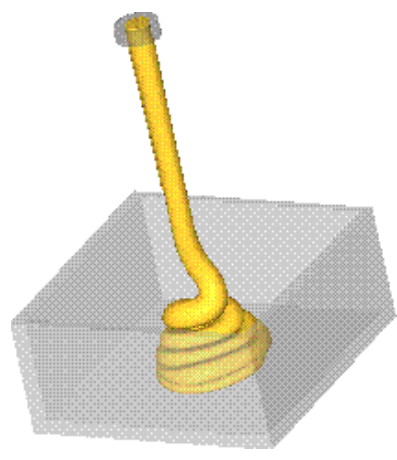

$t=0.40 \mathrm{~s}$

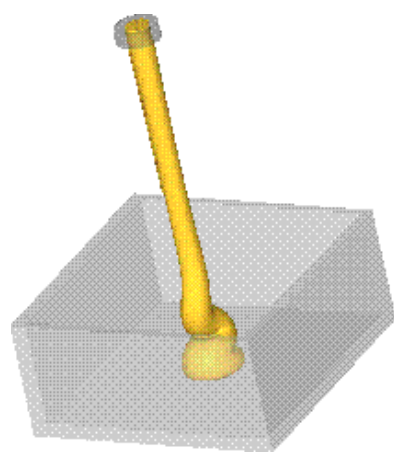

$t=0.20 \mathrm{~s}$

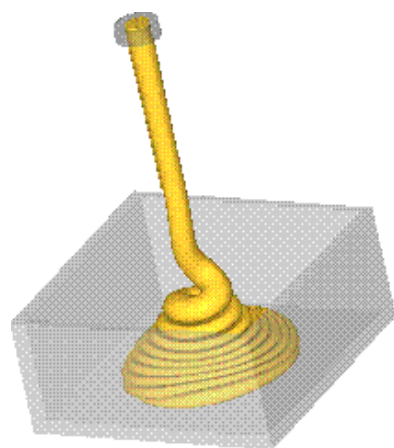

$t=0.70 \mathrm{~s}$

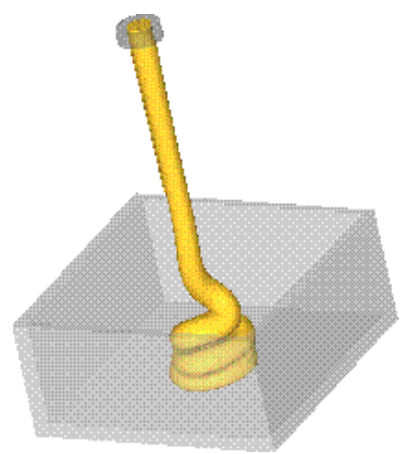

$t=0.30 \mathrm{~s}$

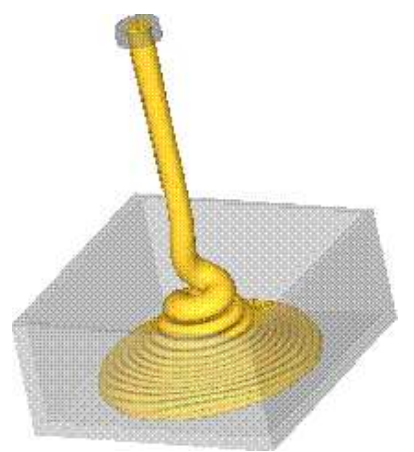

$t=1.00 \mathrm{~s}$

Figura 5.6: Simulação numérica do jato oscilante utilizando a formulação SI-CN/CCI1. Visualização do escoamento em tempos selecionados. ( $R e=0.5$ e $F r=0.396$, fluido newtoniano). 


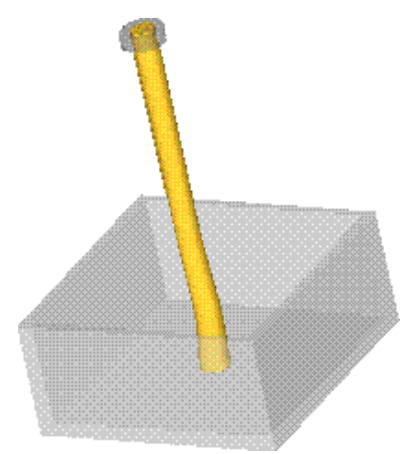

$t=0.14 s$

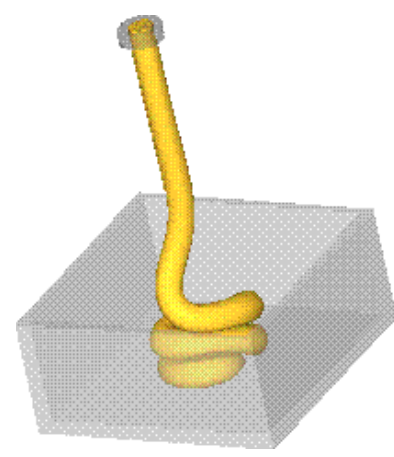

$t=0.40 \mathrm{~s}$

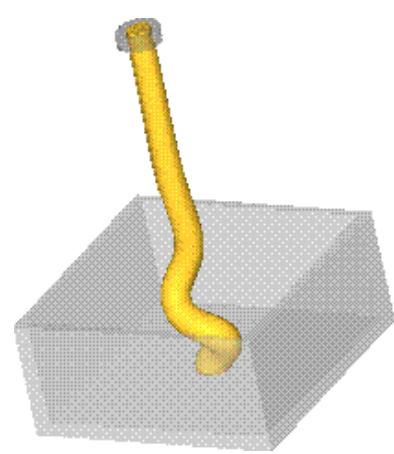

$t=0.20 \mathrm{~s}$

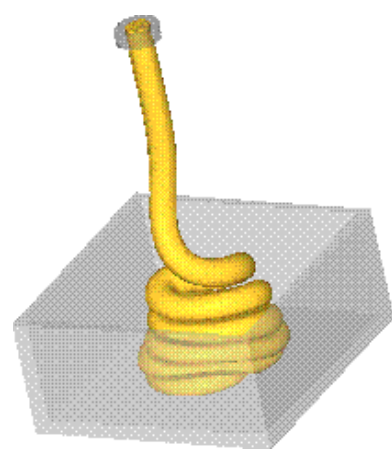

$t=0.70 \mathrm{~s}$

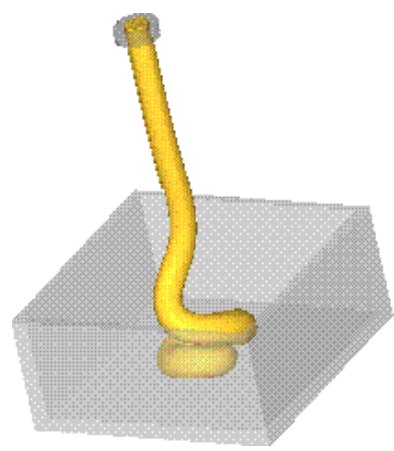

$t=0.30 \mathrm{~s}$

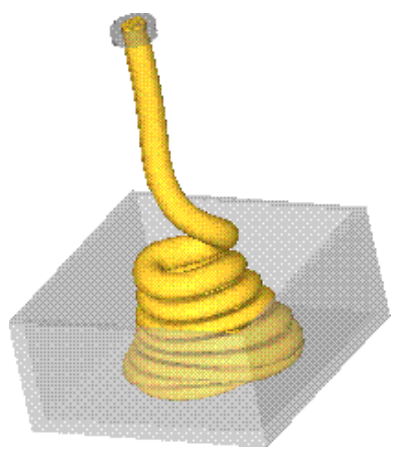

$t=1.00 \mathrm{~s}$

Figura 5.7: Simulação numérica do jato oscilante utilizando a formulação SI-CN/CCI1. Visualização do escoamento em tempos selecionados. ( $R e=0.1$ e $F r=0.396$, fluido newtoniano).

jato oscilante com fluido do tipo Maxwell. Neste caso, foram realizadas duas simulações, uma com o jato axisimétrico e outra com o jato quadrado, utilizando a formulação SI-EI/CCE para fluido do tipo Maxwell e comparadas com os resultados obtidos com um jato newtoniano. Os dados dos modelos implementados no sistema FREEFLOW para estas simulações foram: $\mu=6$ Pa.s, $\rho=1000 \mathrm{~kg} \mathrm{~m}^{-3}$, diâmetro do injetor $(D)$ igual a $6 \mathrm{~mm}$ e altura do injetor até o fundo da caixa $(H)$ igual a $12 \mathrm{~cm}$. Uma caixa de dimensões $7 \mathrm{~cm} \times 7 \mathrm{~cm} \times 3 \mathrm{~cm}$ foi utilizada. No injetor, impõem-se um perfil parabólico (Poiseuille profile) dado pela equação (5.29) com $U=$ $0.5 \mathrm{~m} \mathrm{~s}^{-1}$. Neste caso assume-se que a gravidade está agindo com $g=9.81 \mathrm{~m} \mathrm{~s}^{-2}$. Uma malha de $70 \times 70 \times 128$ células $(\delta x=\delta y=\delta z=1 \mathrm{~mm})$ foi utilizada. Os parâmetros adimensionais são $W e=\frac{\lambda_{1} U}{D}=0.5$ e $R e=\frac{\rho U D}{\mu}=0.5$.

Neste caso tem-se $R e=0.5$ e $\frac{H}{D}=20$, ou seja, de acordo com os resultados experimentais de Cruickshank e Munson [31] as condições $R e=\frac{\rho U D}{\mu}<1.2$ e $\frac{H}{D}>7.2$ são satisfeitas, e portanto espera-se que os jatos apresentem as flambagens. 

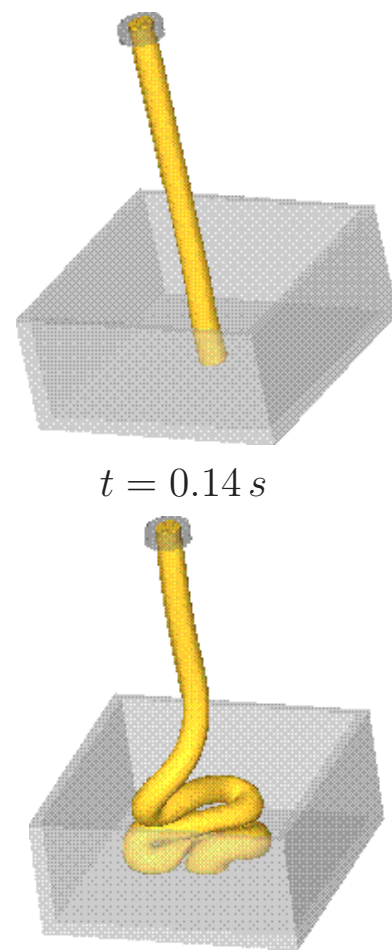

$t=0.40 \mathrm{~s}$

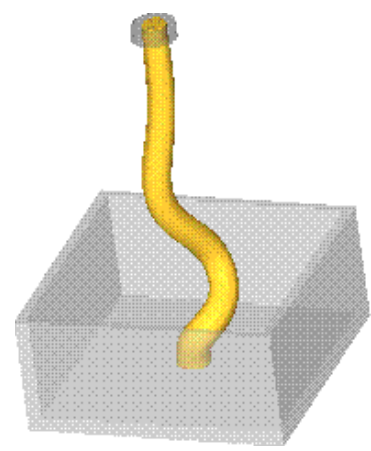

$t=0.20 \mathrm{~s}$

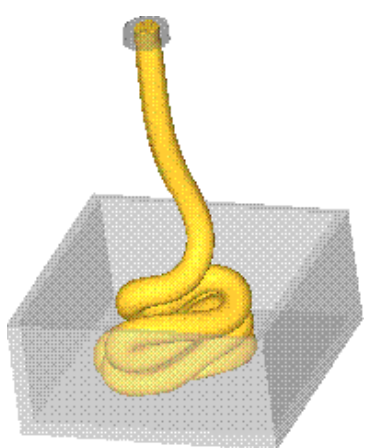

$t=0.70 \mathrm{~s}$

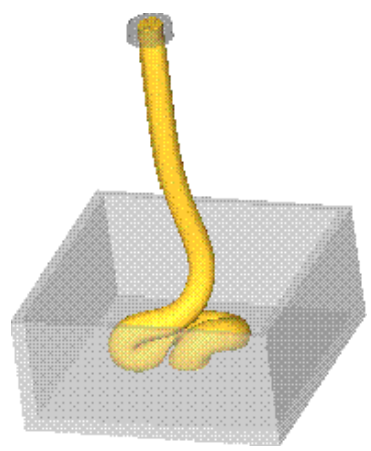

$t=0.30 \mathrm{~s}$

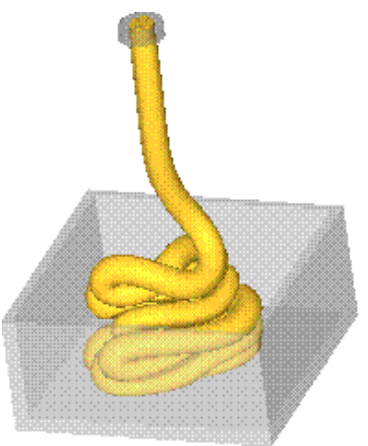

$t=1.00 \mathrm{~s}$

Figura 5.8: Simulação numérica do jato oscilante utilizando a formulação SI-CN/CCI1. Visualização do escoamento em tempos selecionados. ( $R e=0.05$ e $F r=0.396$, fluido newtoniano).

O sistema FREEFLOW simulou estes problemas e os resultados são apresentados nas figuras 5.10 e 5.11. De acordo com as visualizações nas figuras 5.10 e 5.11, nota-se que todos os jatos produziram o efeito de flambagem. Além disso, ambos tipos de fluidos, newtoniano e do tipo Maxwell, apresentaram o efeito coiling para os jatos axisimétrico e quadrado.

Adicionalmente, simulou-se os mesmos problemas, apenas modificando o número de Reynolds para $R e=1.3$ (assumindo $\mu=2.3077$ Pa.s). De acordo com as condições de Cruickshank e Munson [31], neste caso como $R e>1.2$ espera-se que os jatos não apresentem os efeitos de flambagem. De fato, como pode ser observado nas figuras 5.12 e 5.13, os jatos axisimétrico e quadrado, para fluido newtoniano, não apresentaram o fenômeno do jato oscilante. Entretanto, observa-se das figuras 5.12 e 5.13 o efeito da flambagem nos jatos para fluido do tipo Maxwell (esse fato é mais evidente no jato quadrado, figura 5.13). Acredita-se que este fato ocorreu devido a alta viscosidade existensional desenvolvida depois que o jato atinge a superfície rígida. Este fato foi discutindo em Tomé et al. [165]. 


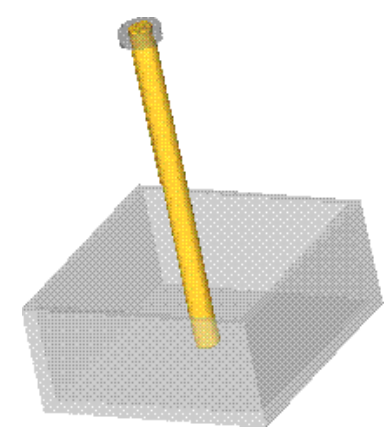

$t=0.14 \mathrm{~s}$

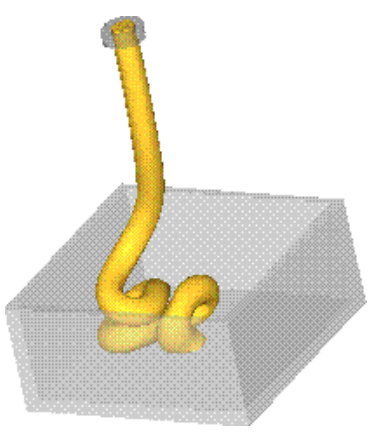

$t=0.40 s$

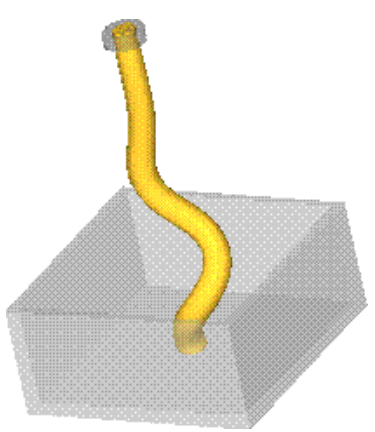

$t=0.20 \mathrm{~s}$

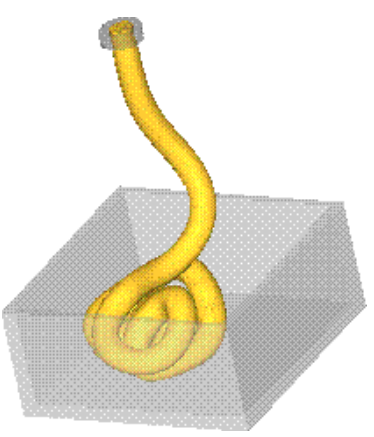

$t=0.70 \mathrm{~s}$

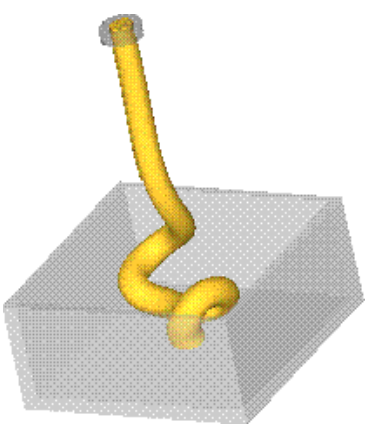

$t=0.30 \mathrm{~s}$

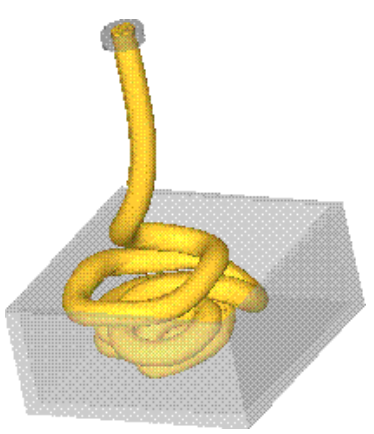

$t=1.00 \mathrm{~s}$

Figura 5.9: Simulação numérica do jato oscilante utilizando a formulação SI-CN/CCI1. Visualização do escoamento em tempos selecionados. ( $R e=0.01$ e $F r=0.396$, fluido newtoniano).

\subsubsection{Simulação numérica do inchamento do extrudado}

O fenômeno do inchamento do extrudado (extrudate swell phenomenon) é geralmente associado com fluidos viscoelásticos (em fluidos newtonianos este inchamento não é tão considerável como foi mostrado no caso bidimensional na seção 4.5) em processos de extrussão. Neste problema, um jato escoa dentro de um tubo de diâmetro $D$ e na saída do tubo o jato é expelido para a atmosfera. Devido às diferenças de tesão normal, o diâmetro do jato tende a aumentar para $D_{\max }$. O inchamento do extrudado é usualmente associado com escoamentos a baixo número de Reynolds e tem sido estudado por muitos pesquisadores. Por exemplo, alguns resultados experimentais para fluidos viscoelásticos foram descritos por Liang et al. [92], Liang [91], Sombatsompop e Intawong [144], enquanto que resultados numéricos podem ser encontrados na literatura em Mitsoulis [101], Rutgers e Mackley [139], Huang e Lu [75], Paulo et al. [123], Tomé et al. [160].

Para demonstrar que as técnicas implícitas apresentadas neste trabalho podem simular escoamentos de fluidos altamente viscoelásticos com superfície livre a baixo número de Reynolds, 


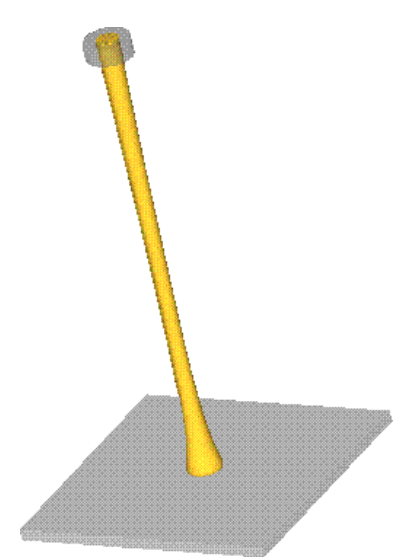

$t=0.175 s$

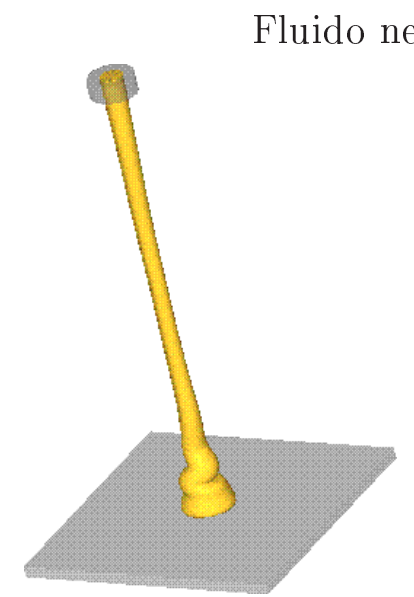

$t=0.25 \mathrm{~s}$

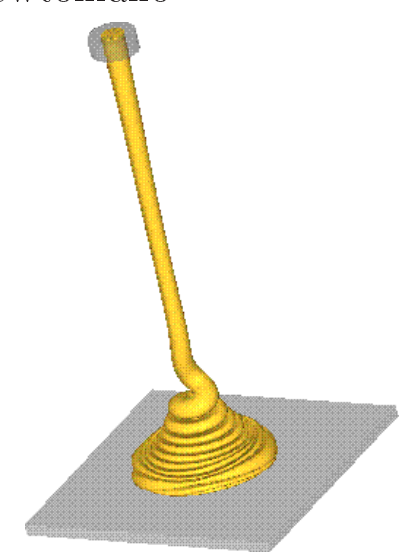

$t=0.75 \mathrm{~s}$

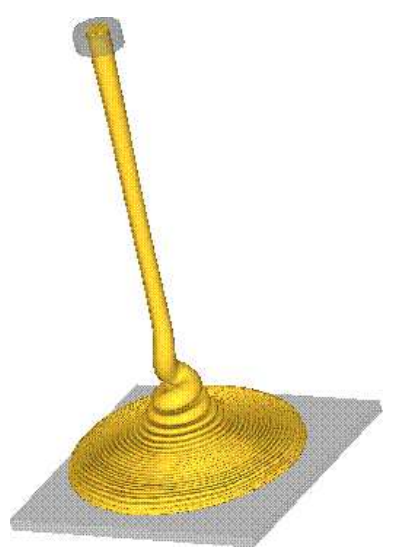

$t=1.625 \mathrm{~s}$

Formulação SI-EI/CCE com fluido do tipo Maxwell

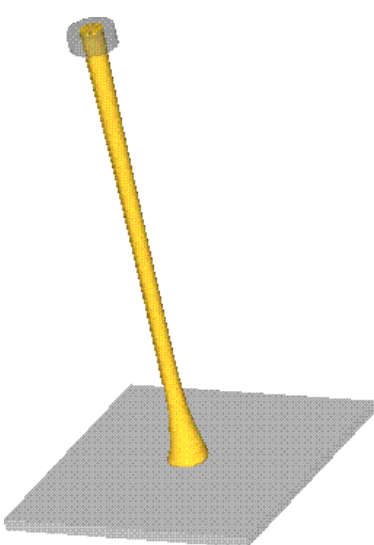

$t=0.175 \mathrm{~s}$

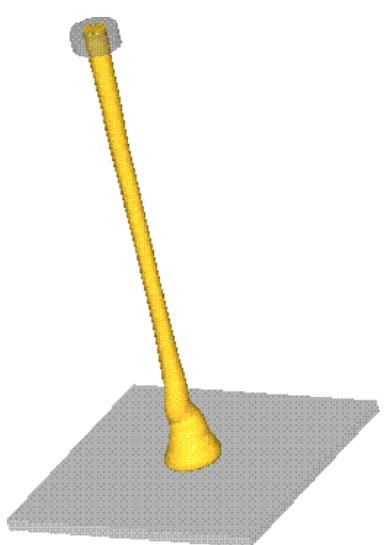

$t=0.25 \mathrm{~s}$

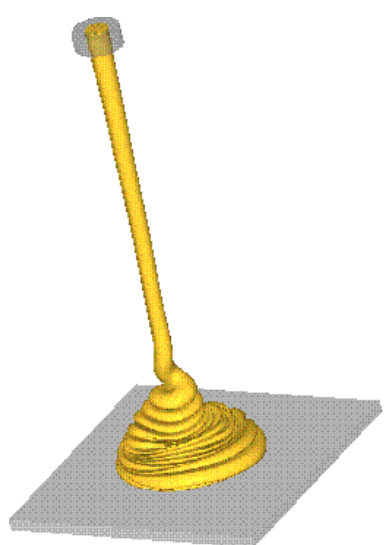

$t=0.75 \mathrm{~s}$

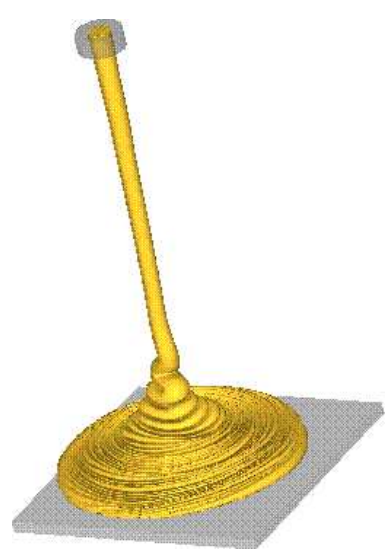

$t=1.625 \mathrm{~s}$

Figura 5.10: Simulação numérica do jato oscilante axisimétrico de um fluido newtoniano e do tipo Maxwell utilizando a formulação implícita (SI-EI/CCE, descrita na seção 5.2). Visualização do escoamento em tempos selecionados. $(R e=0.5$ e $W e=0.5)$.

estudou-se o fenômeno do inchamento do extrudado de fluidos tipo Oldroyd-B e Maxwell com diferentes números de Weissenberg. Para isto, considerou-se um tubo circular de comprimento $5 R$, descrito anteriormente (ver figura 5.2), e especificou-se uma condição de contorno para o ejetor localizado a uma distância de $5 R$ da saída do tubo. Na entrada do tubo, impõem-se as soluções dadas pelas equações (5.29) e (5.32) no caso do fluido Oldroyd-B, enquanto que para o Maxwell utiliza-se as soluções de (5.29) e (5.31). As condições de contorno na parede rígida foram apresentadas em (2.4). 


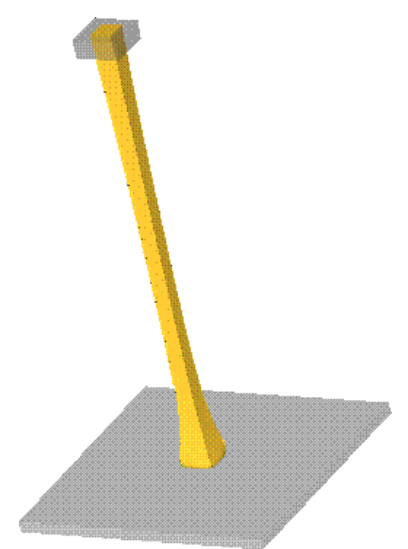

$t=0.175 \mathrm{~s}$

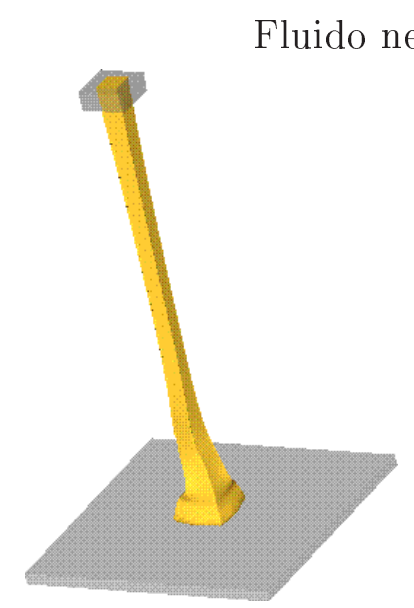

$t=0.25 \mathrm{~s}$

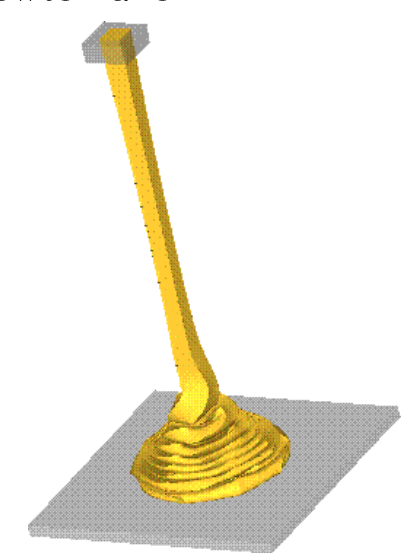

$t=0.75 \mathrm{~s}$

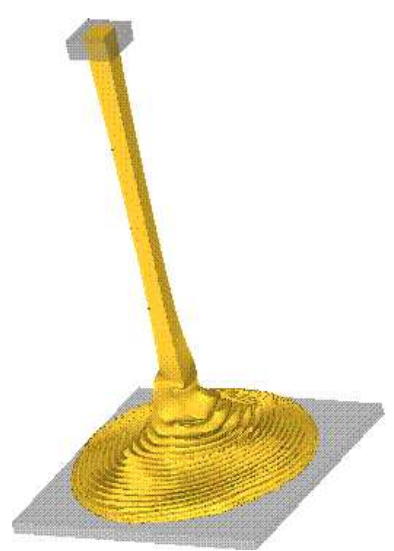

$t=1.50 \mathrm{~s}$

Formulação SI-EI/CCE com fluido do tipo Maxwell

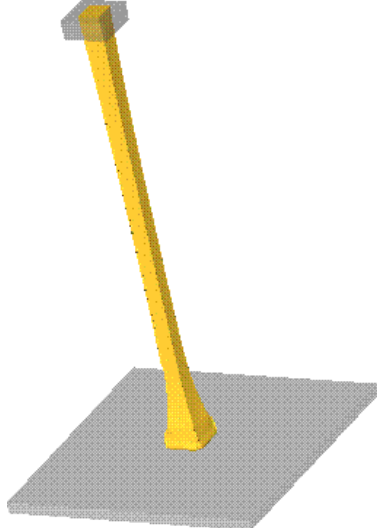

$t=0.175 s$

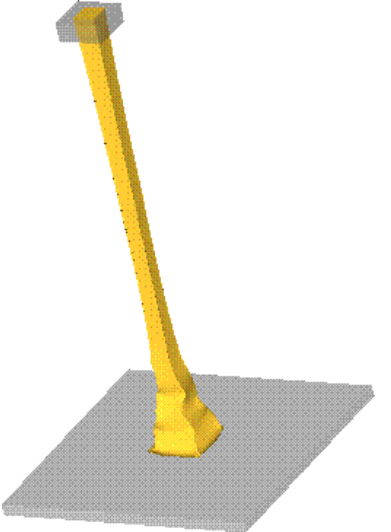

$t=0.25 \mathrm{~s}$

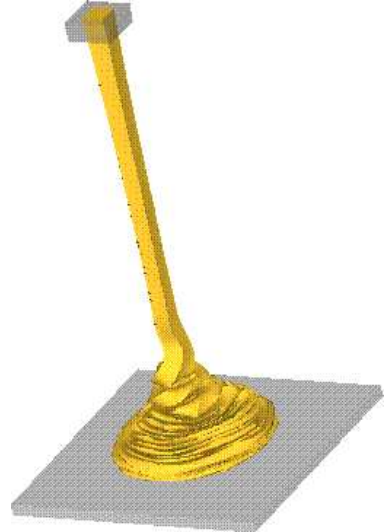

$t=0.75 \mathrm{~s}$

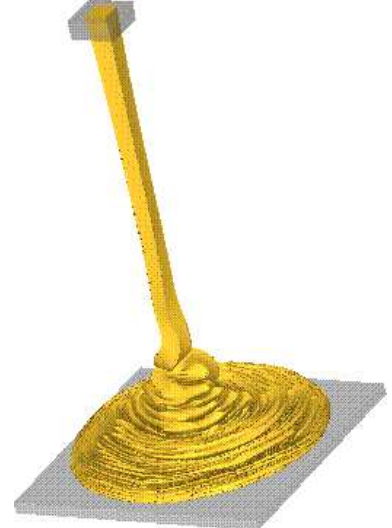

$t=1.50 \mathrm{~s}$

Figura 5.11: Simulação numérica do jato oscilante quadrado de um fluido newtoniano e do tipo Maxwell utilizando a formulação implícita (SI-EI/CCE, descrita na seção 5.2). Visualização do escoamento em tempos selecionados. ( $R e=0.5$ e $W e=0.5)$.

As primeiras simulações foram realizadas utilizando a formulação SI-CN. Neste caso, utilizouse uma malha de $16 \times 16 \times 80$ células $(\delta x=\delta y=\delta z=0.625 \mathrm{~mm})$ e os seguintes parâmetros de entrada: $R=5 \mathrm{~mm}$, a força da gravidade agindo na direção $z$ com $g=9.81 \mathrm{~m} \mathrm{~s}^{-2}, \rho=1000 \mathrm{~kg} \mathrm{~m}^{-3}$, e $\lambda_{1}=0.01 \mathrm{~s}$. Os parâmetros de escala foram $L=R, U=0.5 \mathrm{~m} \mathrm{~s}^{-1}$ resultando em $R e=0.1$ (para $\mu=25$ Pa.s) e $R e=0.05$ (para $\mu=50$ Pa.s), e em ambos os casos $W e=1$. Quando $R e=0.1$ utiliza-se $\delta t=6.5 \times 10^{-6} s$, enquanto que para $R e=0.05$ tem-se $\delta t=3.25 \times 10^{-6} s$.

Para demonstrar que a formulação SI-CN pode reproduzir efeitos de viscoelásticidade, simulou-se o inchamento do extrudado para três valores do número de Weissenberg efetivo, 


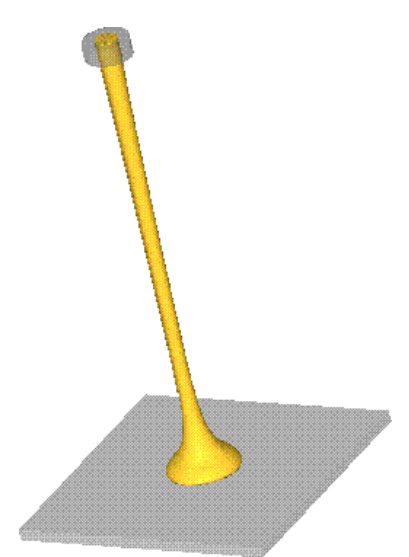

$t=0.175 \mathrm{~s}$

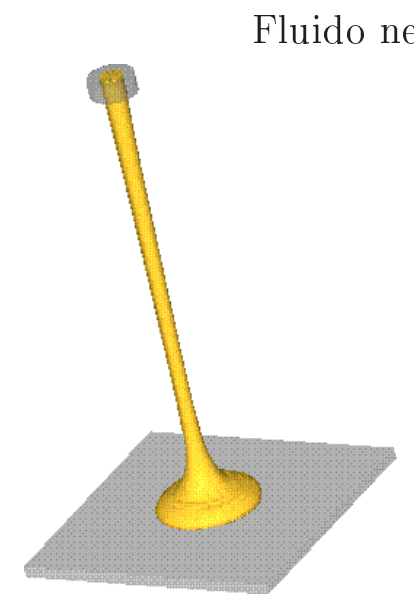

$t=0.25 \mathrm{~s}$

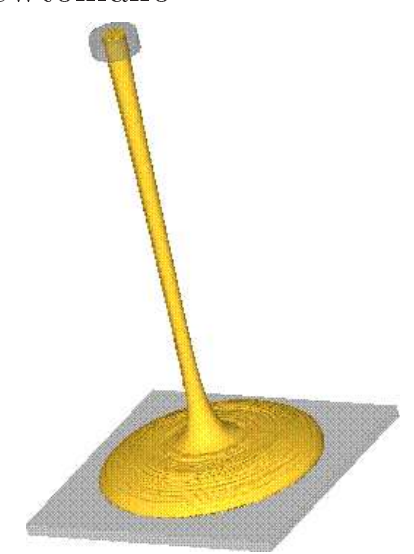

$t=0.75 s$

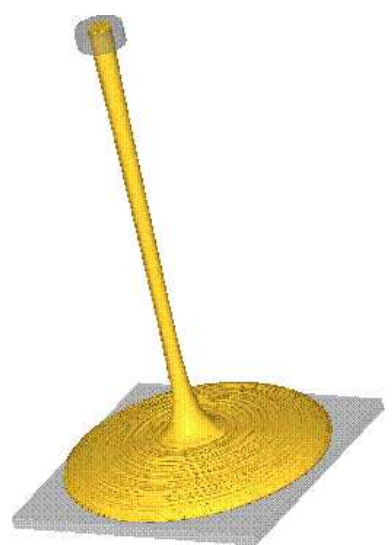

$t=1.50 \mathrm{~s}$

Formulação SI-EI/CCE com fluido do tipo Maxwell

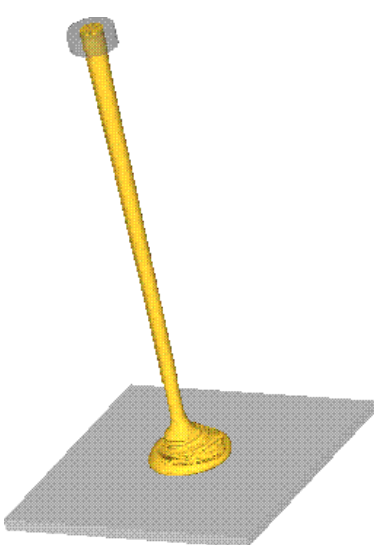

$t=0.175 \mathrm{~s}$

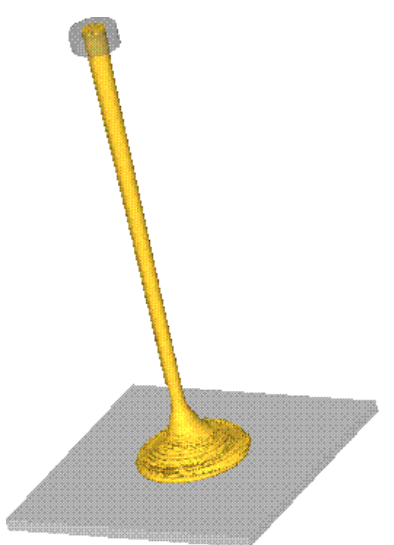

$t=0.25 \mathrm{~s}$

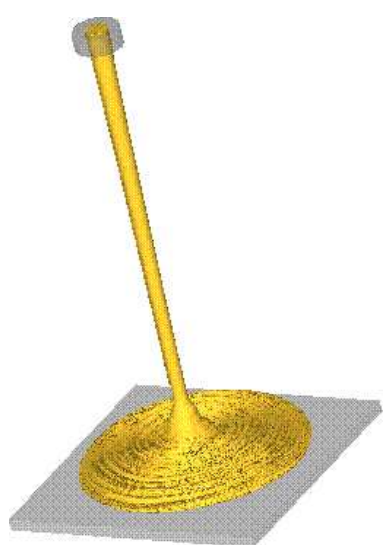

$t=0.75 \mathrm{~s}$

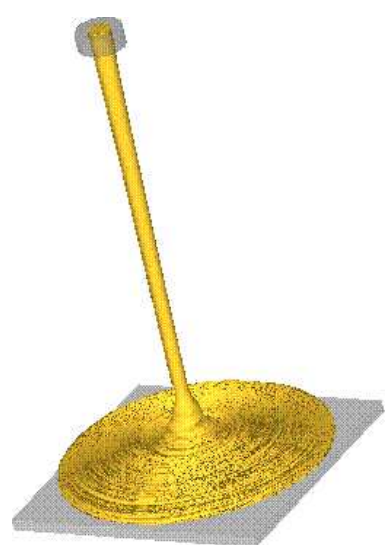

$t=1.50 \mathrm{~s}$

Figura 5.12: Simulação numérica do jato oscilante axisimétrico de um fluido newtoniano e do tipo Maxwell utilizando a formulação implícita (SI-EI/CCE, descrita na seção 5.2). Visualização do escoamento em tempos selecionados. $(R e=1.3$ e $W e=0.5)$.

definido como $W e_{\text {efetivo }}=\left(1-\lambda_{2} / \lambda_{1}\right) W e$. Para produzir estes valores do $W e_{\text {efetivo foram }}$ escolhidos $\lambda_{2}=0.008 s, 0.005 s, 0.002 s$ resultando em $W e_{\text {efetivo }}=0.2,0.5,0.8$, respectivamente.

Inicialmente o fluido entra no tubo vazio, preenchendo-o e então o jato de fluido escapa para a atmosfera até atingir o ejetor. Estas simulações foram executadas até $t=0.45 \mathrm{~s}$ em todos os casos. Os resultados são apresentados na figura 5.14, a qual apresenta a configuração do fluido em diferentes tempos. No tempo $t=0.10 \mathrm{~s}$, os jatos estão saindo do tubo e o campo de velocidade calculado para os três valores de $W e_{\text {efetivo }}$ são semelhantes. Entretanto, no tempo $t=0.20 \mathrm{~s}$ já é possível verificar diferenças entre as três simulações. Pode-se observar que o 


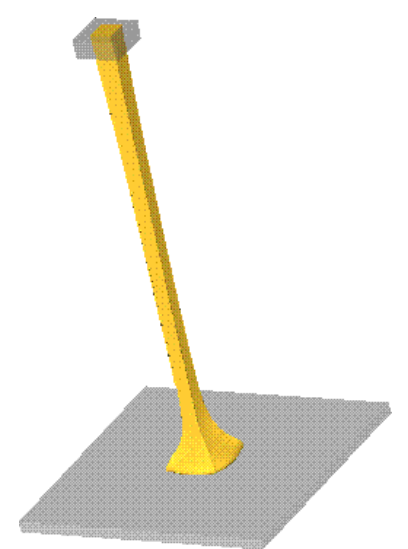

$t=0.175 \mathrm{~s}$

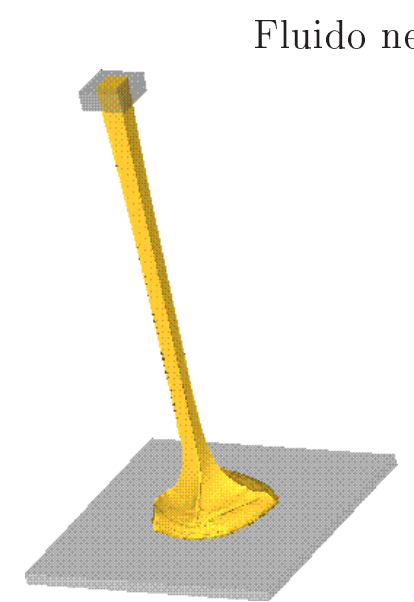

$t=0.25 \mathrm{~s}$

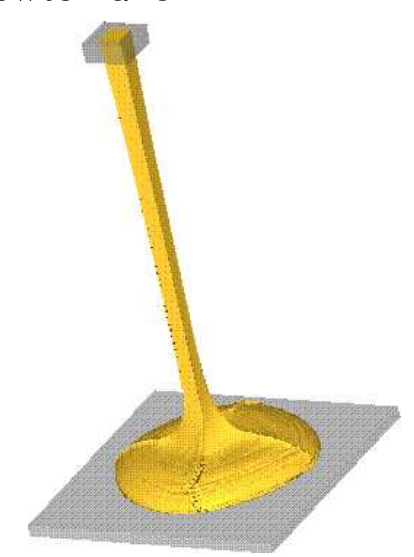

$t=0.75 \mathrm{~s}$

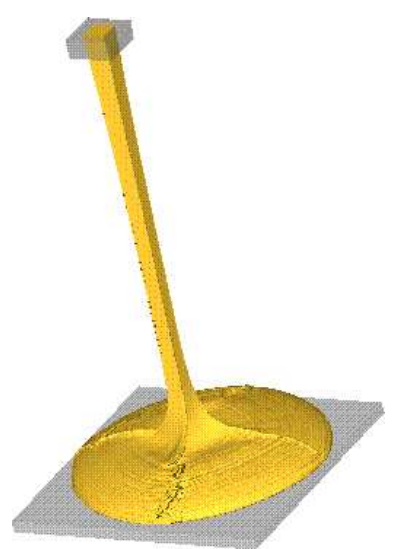

$t=1.50 \mathrm{~s}$

Formulação SI-EI/CCE com fluido do tipo Maxwell

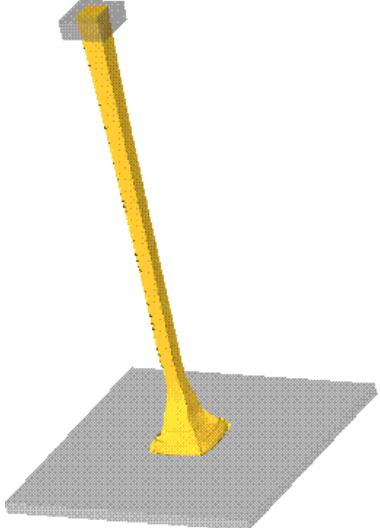

$t=0.175 \mathrm{~s}$

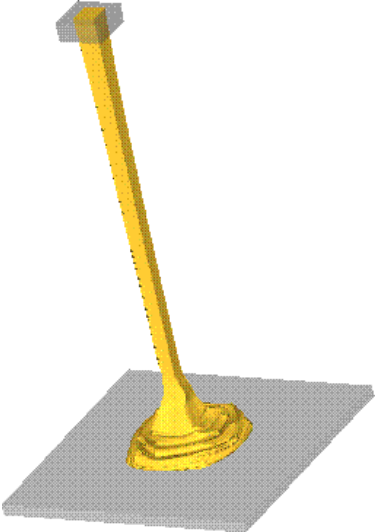

$t=0.25 \mathrm{~s}$

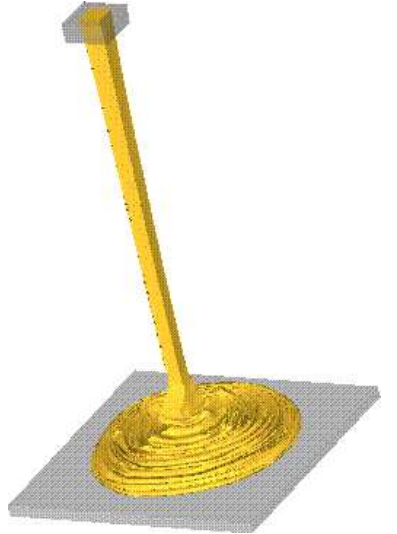

$t=0.75 \mathrm{~s}$

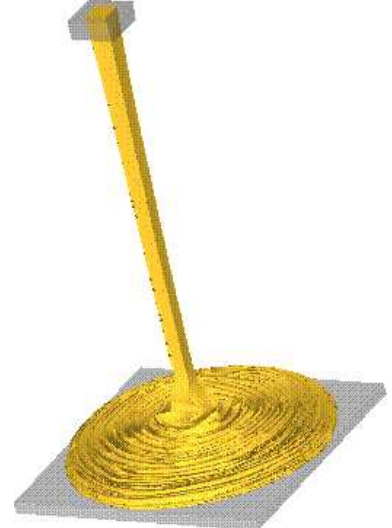

$t=1.50 \mathrm{~s}$

Figura 5.13: Simulação numérica do jato oscilante quadrado de um fluido newtoniano e do tipo Maxwell utilizando a formulação implícita (SI-EI/CCE, descrita na seção 5.2). Visualização do escoamento em tempos selecionados. $(R e=1.3$ e $W e=0.5)$.

jato com $W e_{\text {efetivo }}=0.8$ apresenta um inchamento maior que os jatos com $W e_{\text {efetivo }}=0.5 \mathrm{e}$ $W e_{\text {efetivo }}=0.2$, os quais estão iniciando o inchamento. No tempo $t=0.30 \mathrm{~s}$, observa-se que, devido ao efeito da gravidade, os jatos são atraídos em direção ao ejetor. De fato, neste tempo, o jato com $W e_{\text {efetivo }}=0.2$ já atingiu o ejetor. A ultima linha da figura 5.14 apresenta os resultados obtidos no tempo $t=0.45 s$ para as três simulações, e pode-se observar claramente o efeito da gravidade nos jatos. Entre os tempos $t=0.4 \mathrm{~s}$ e $t=0.45 \mathrm{~s}$ não ocorreram grandes variações nos resultados, e portanto, pode-se assumir que os escoamentos já encontram-se em estado estacionário. 
A razão de inchamento do extrudado, definido como

$$
S_{r}=D_{\max } / D
$$

foi $42.04 \%$ para $W e_{\text {efetivo }}=0.2,65.15 \%$ para $W e_{\text {efetivo }}=0.5$ e $76.47 \%$ para $W e_{\text {efetivo }}=0.8$.

Além destas simulações do fenômeno do inchamento do extrudado, foram realizadas outras simulações, com os mesmos parâmetros anteriores, exceto para o número de Reynolds que foi tomado como $R e=0.05$. Os resultados obtidos (ver figura 5.15) são semelhantes aos resultados para $R e=0.1$; a diferença mais notável entre os dois casos foi o aumento na razão de inchamento do extrudado. A razão de inchamento do extrudado neste caso foi $45.71 \%$ para $W e_{\text {efetivo }}=0.2$, $73.91 \%$ para $W e_{\text {efetivo }}=0.5$ e $85.71 \%$ para $W e_{\text {efetivo }}=0.8$. Os resultados para $R e=0.1 \mathrm{e}$ $R e=0.05$ estão em concordância com os resultados de Liang et al. [92].

Para o fluido do tipo Maxwell, utilizou-se a formulação SI-EI, descrita na seção 5.2. Neste caso, utilizou-se uma malha de $48 \times 48 \times 160$ células $(\delta x=\delta y=\delta z=0.625 \mathrm{~mm})$ e os seguintes parâmetros de entrada: $R=0.5 \mathrm{~cm}, \rho=1000 \mathrm{~kg} \mathrm{~m}^{-3}$, e $\lambda_{1}=0.001,0.002,0.004 \mathrm{~s}$. Os parâmetros de escala foram $L=R, U=0.5 \mathrm{~m} \mathrm{~s}^{-1}$ resultando em $R e=1$ (para $\mu=2.5$ Pa.s). Os valores do número de Weissenberg foram calculados de acordo com a variação do parâmetro $\lambda_{1}=0.001,0.002,0.004 \mathrm{~s}$, resultando em $W e=\frac{\lambda_{1} U}{R}=0.1,0.2$ e 0.4 , respectivamente. De acordo com os dados utiliza-se $\delta t=2.60 \times 10^{-5} s$.

Os resultados destas simulações estão apresentados na figura 5.16, onde nota-se que quando o número $W e$ aumenta, a razão de inchamento $S_{r}$ dada por (5.33), também aumenta. Para o fluido do tipo Maxwell, um valor teórico foi apresentado por Tanner [149]. A lei de Tanner, que considera a razão de inchamento sem levar em consideração a ação da gravidade, é dada por $S_{r}=0.13+\left[1+8 W e^{2}\right]^{1 / 6}$. A tabela 5.4 apresenta os resultados obtidos pela formulação SI-EI e os resultado dados pela lei de Tanner. De acordo com os valores na tabela 5.4, nota-se concordância entre os resultados numéricos e os resultados de Tanner [149]. Além disso, os resultados obtidos com a formulação SI-EI estão relativamente próximos aos valores obtidos por Tomé et al. [160].

Tabela 5.4: Resultados do escoamento do inchamento do extrudado para um fluido do tipo Maxwell. Os valores numéricos de $S_{r}$ foram obtidos pela formulação SI-EI/CCE, enquanto que os teóricos pela lei de Tanner [149].

\begin{tabular}{|r|c|c|c|}
\hline & $W e=0.1$ & $W e=0.2$ & $W e=0.4$ \\
\hline$S_{r}$ (SI-EI/CCE) & 1.113 & 1.188 & 1.375 \\
$S_{r}$ (lei de Tanner) & 1.143 & 1.177 & 1.277 \\
\hline
\end{tabular}


5.4 Aplicações

97

$$
t=0.10 s
$$
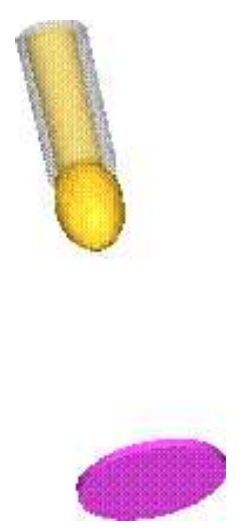

$t=0.20 s$
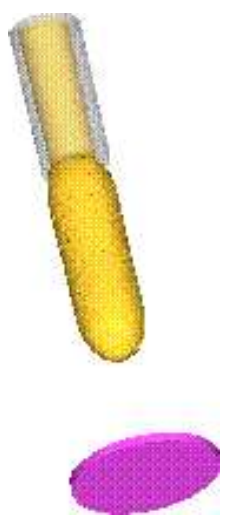

$t=0.30 \mathrm{~s}$

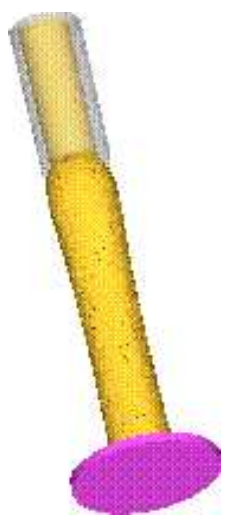

$t=0.45 \mathrm{~s}$

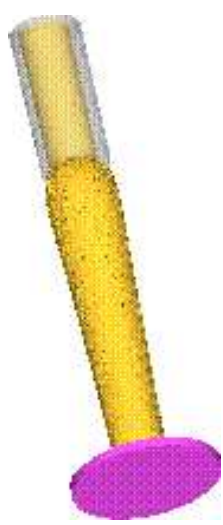

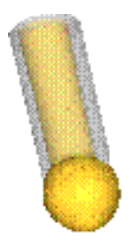
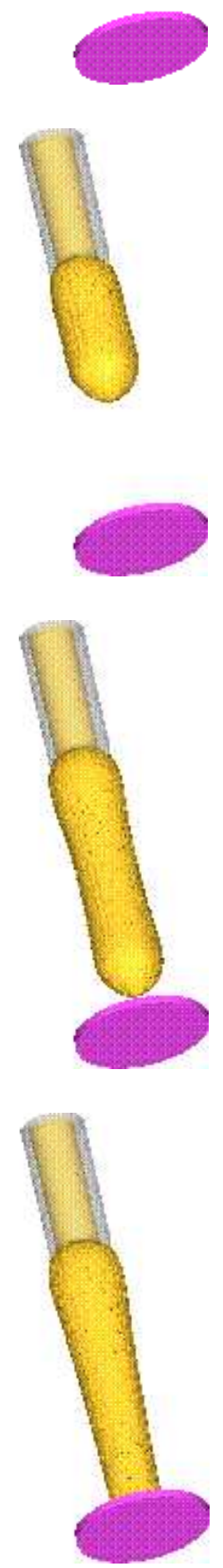
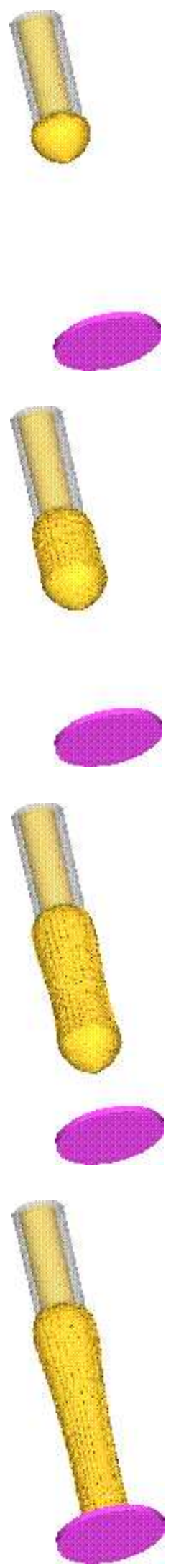

Figura 5.14: Simulação numérica do fenômeno do inchamento do extrudado não-estacionário para vários valores de $W e_{\text {efetivo }}$ : 0.2 (primeira coluna); 0.5 (segunda coluna); 0.8 (terceira coluna). Visualização do escoamento em tempos selecionados ( $R e=0.1$, fluido do tipo Oldroyd$\mathrm{B})$. 
98

Métodos de projeção 3D

$$
t=0.10 s
$$
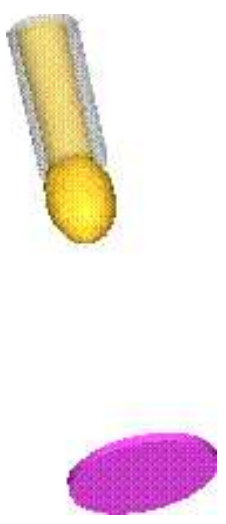

$$
t=0.20 \mathrm{~s}
$$
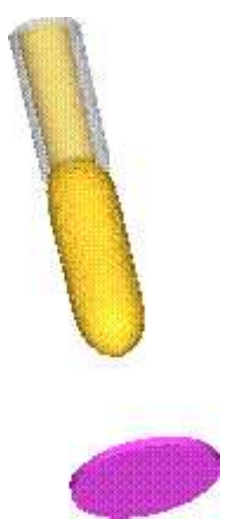

$t=0.30 \mathrm{~s}$

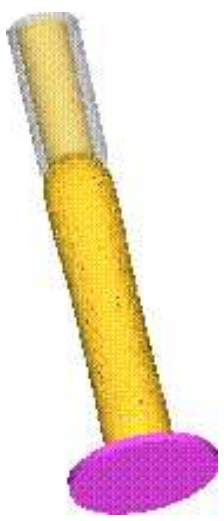

$t=0.45 s$

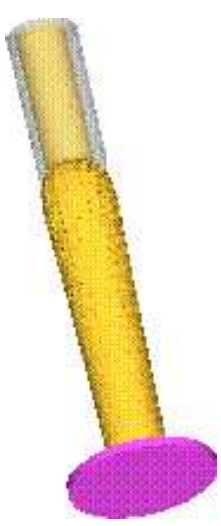

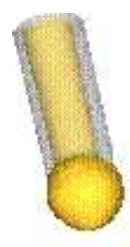
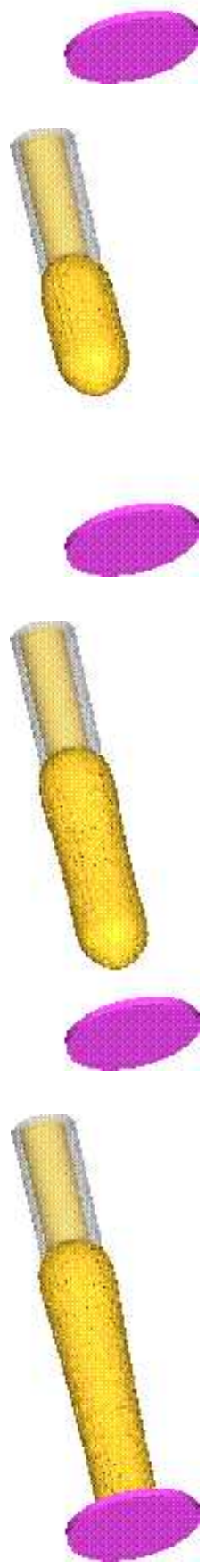
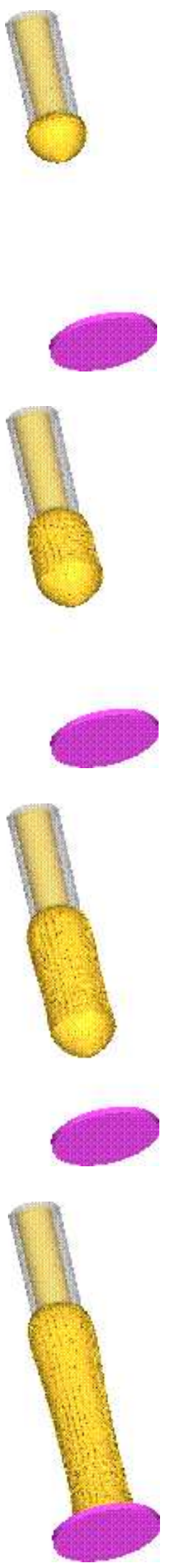

Figura 5.15: Simulação numérica do fenômeno do inchamento do extrudado não-estacionário para vários valores de $W e_{\text {efetivo }}$ : 0.2 (primeira coluna); 0.5 (segunda coluna); 0.8 (terceira coluna). Visualização do escoamento em tempos selecionados ( $R e=0.05$, fluido do tipo OldroydB). 
$t=0.05 \mathrm{~s}$
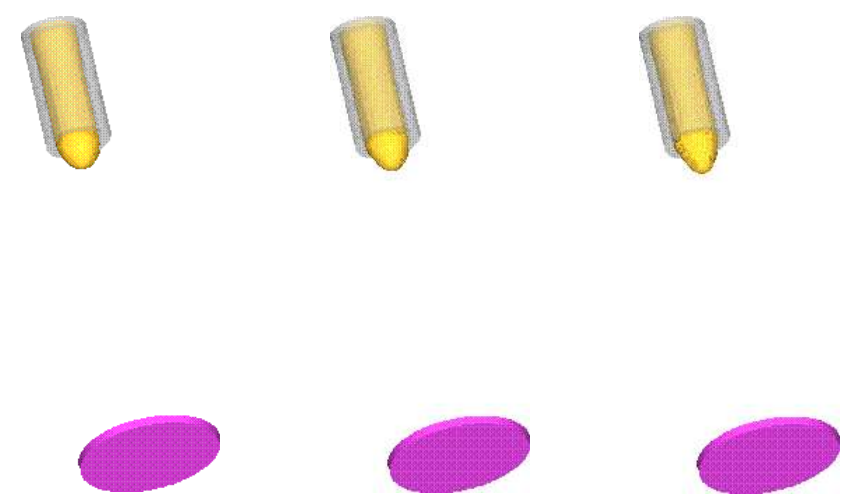

$t=0.10 \mathrm{~s}$
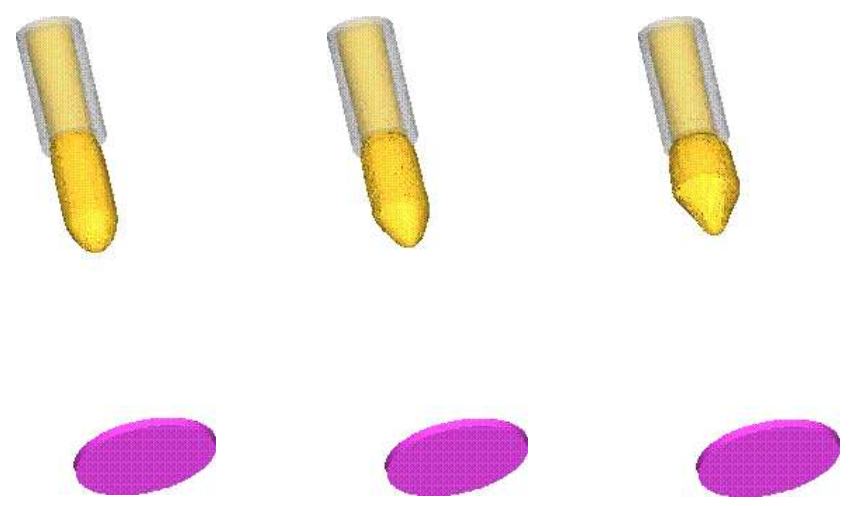

$t=0.15 s$
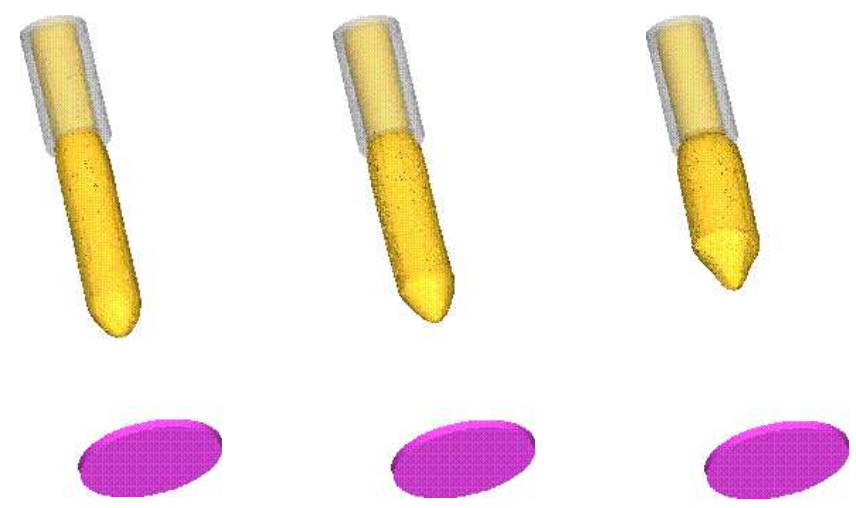

$t=0.20 \mathrm{~s}$
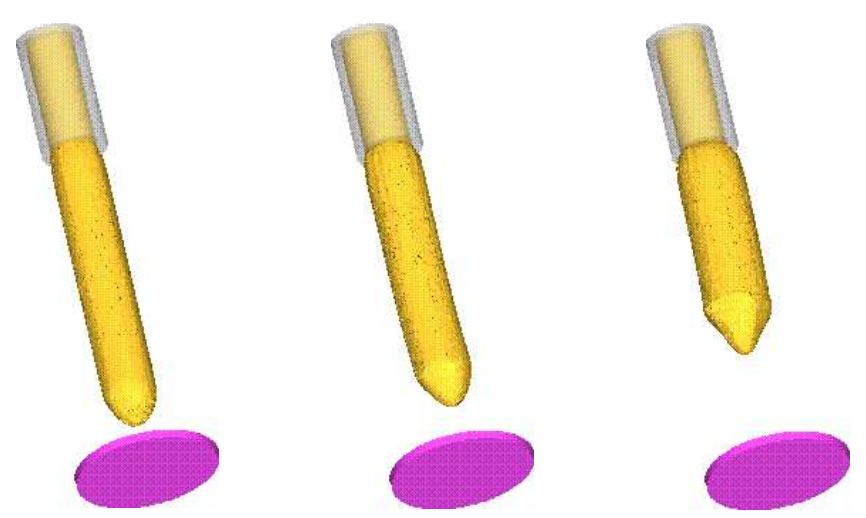

Figura 5.16: Simulação numérica do fenômeno do inchamento do extrudado não-estacionário para vários valores de $W e: 0.1$ (primeira coluna); 0.2 (segunda coluna); 0.4 (terceira coluna). Visualização do escoamento em tempos selecionados ( $R e=1.0$, fluido do tipo Maxwell). 


\subsection{Considerações finais}

Neste capítulo, as técnicas introduzidas no capítulo 4 foram estendidas para escoamentos tridimensionais, possibilitando a construção de um método semi-implícito generalizado. A equação de quantidade de movimento e as equações para a pressão tentativa $\psi$ na superfície livre foram derivadas de forma a possibilitar a utilização deste método em escoamentos tridimensionais newtonianos e viscoelásticos.

No caso de escoamentos viscoelásticos, os modelos diferenciais escolhidos foram o de Maxwell e Oldroyd-B. Assim como no caso bidimensional em escoamentos newtonianos, a formulação tridimensional apresentada é estável e não necessita da restrição parabólica do passo do tempo. Além disso, para os modelos de Maxwell e Oldroyd-B, o método de projeção descrito neste capítulo foi capaz de capturar efeitos de viscoelásticidade utilizando passos no tempo bem maiores que no método explícito.

Os resultados numéricos para o caso tridimensional foram apresentados nas seções 5.3 e 5.4 . Deve-se ressaltar, que neste capítulo não foi detalhado os modelos de Maxwell e Oldroyd-B, pois o objetivo principal deste trabalho é mostrar que as novas técnicas implícitas podem ser aplicadas tanto para fluidos newtonianos, como em fluidos viscoelásticos. As equações utilizadas foram descritas em [143, 165] no caso do fluido de Maxwell e em [157] para o modelo Oldroyd-B.

Os resultados numéricos apresentaram boa concordância com soluções exatas e conhecidas. O refinamento de malha indicou a convergência dos esquemas implícitos. Todas as simulações foram realizadas para problemas a baixo número de Reynolds, o que indica que as técnicas desenvolvidas neste capítulo são capazes de resolver os escoamentos conhecidos como creeping flow. Os resultados deste capítulo foram publicados em Oishi et al. [118]. 


\section{Métodos de projeção de segunda ordem para escoamentos com superfície livre}

Os métodos de projeção apresentados anteriormente demonstraram eficiência e robustez em escoamentos com superfície livre a baixo número de Reynolds. Além disso, os esquemas foram capazes de capturar efeitos em escoamentos newtonianos e viscoelásticos. Porém, até o momento, não foram realizados testes sobre a ordem de convergência temporal destas formulações. Portanto cabe mais uma pergunta: "É possível construir um método de projeção para escoamentos com superfície livre a baixo número de Reynolds de ordem maior que um ? A presente tese encerrará com a resposta para esta pergunta.

\subsection{Introdução}

As técnicas desenvolvidas neste trabalho, utilizam a metodologia do desacoplamento para simplificar a solução das equações de Navier-Stokes com condições de contorno de superfície livre. Nos capítulos 4 e 5 foram discutidas formulações que utilizam a condição do tensor normal à superfície livre discretizada implicitamente. Uma nova estratégia para desacoplar os campos de velocidade e pressão, tanto para as equações de Navier-Stokes, como na equação da pressão na superfície livre foi proposta. Entretanto, o preço do desacoplamento das equações é a perda de precisão temporal da solução, que é avaliada pelo Erro de Truncamento Local (ETL). 
Geralmente, qualquer equação discretizada no tempo e/ou no espaço não é exatamente satisfeita pela solução teórica da equação diferencial parcial, e essa discrepância define o ETL. Assim, a precisão de um esquema numérico é medida pela rapidez com que o ETL tende a zero.

Os trabalhos pioneiros de Harlow e Welch [66], Chorin [22, 23] e Temam [152] apresentaram métodos de projeção de primeira ordem de precisão temporal. O estudo do ETL de métodos do tipo Chorin [22, 152] pode ser encontrado, por exemplo, nos trabalhos de Gresho [59], Shen [140], Weton [175], entre outros. Os métodos do tipo Chorin, chamados por Gresho [59] de métodos de projeção $P 1$, utilizam a equação de quantidade de movimento (2.1) para obter uma velocidade intermediária, porém o gradiente de pressão é eliminado desta equação. Veja no Apêndice A, seção A.1, o efeito da eliminação do gradiente de pressão na equação de quantidade de movimento.

Nas últimas décadas, desde a introdução do conceito de métodos de projeção no final dos anos 60, tem havido um esforço enorme de muitos pesquisadores na análise, extensão e implementação de várias versões dos métodos de projeção. As muitas variações do esquema original de Chorin objetivam a obtenção de métodos de projeção de segunda ordem. Entretanto, para a obtenção de um método de projeção de ordem dois, alguns aspectos polêmicos são constantemente discutidos na literatura. Geralmente, esses aspectos estão relacionados com as possíveis fontes de erro na construção de um método. Os tipos de erros mais discutidos são:

- Erros devidos à atualização da pressão e da velocidade;

- Erros provenientes do tratamento das condições de contorno;

- Erros intrisicamente relacionados ao desacoplamento do sistema original das equações de Navier-Stokes.

Portanto, para a construção de métodos de projeção de segunda ordem de precisão temporal é necessário um estudo dos fatores citados acima.

A atualização da pressão é um passo importante na construção de métodos de projeção de alta ordem temporal. Desta forma, esse passo tem sido muito discutido na literatura, e muitas vezes há alguma discordância entre os autores. Os esquemas que exploram formas diferentes na atualização da pressão são conhecidos como métodos de "correção da pressão". Autores como Van Kan [78], Bell et al. [11], Karniadakis et al. [79], Tau [151], Timmermans et al. [154], Shen [141], Strilkwerda e Lee [146], Brown et al. [13], Guermond e Shen [62], Pyo e Shen [132] estudaram e desvendaram os efeitos dos esquemas de atualização da pressão em métodos de projeção de ordem dois. Recentemente, outra estratégia de atualização apareceu 
na literatura e ficou conhecida como métodos de "correção da velocidade". A noção de esquemas do tipo correção da velocidade em métodos de projeção foi primeiro introduzido por Guermond e Shen [63]. Neste tipo de esquema, os termos viscosos são tratados explicitamente num passo inicial e a velocidade é corrigida no próximo passo. De acordo com Guermond e Shen em outro trabalho [62], os esquemas do tipo correção-velocidade são mais estáveis que os esquemas do tipo correção-pressão. No trabalho de Guy e Fogelson [65], a estabilidade de métodos de projeção de segunda ordem de precisão temporal é analisada. Os resultados mostram que métodos de projeção de alta ordem podem se tornar muito sensíveis a instabilidades numéricas conforme o esquema utilizado na correção da pressão. Codina [24] também apresentou uma análise da estabilidade para a pressão em métodos de primeira e segunda ordem. Mais detalhes sobre o estudo da estabilidade de métodos de projeção podem ser encontrados em [2].

Outra fonte de ambiguidade em métodos de projeção de alta ordem é o tratamento das condições de contorno. Quando a idéia dos métodos de projeção é aplicada, a equação de quantidade de movimento (2.1) é utilizada para calcular um campo de velocidade intermediária, e no próximo passo, uma equação do tipo Poisson para uma pressão intermediária é resolvida. Essas quantidades intermediárias são usadas para calcular as quantidade físicas, velocidade e pressão. Um problema é que as quantidades intermediárias não são quantidades físicas, e portanto não são dadas a priori na formulação do problema, sendo determinadas após o desacoplamento das equações de Navier-Stokes (2.1) e (2.2). Desta forma, como no caso da correção da pressão, tem havido muita discussão sobre o efeito das condições de contorno na precisão temporal dos métodos de projeção. Autores como Fortin et al. [51], Deville et al. [36], Orszag et al. [120], Gresho [59], Perot [126], Weinan e Liu [174], Cortez [26], E e Liu [40] e mais recentemente Strilkwerda e Lee [146], Brown et al. [13], Lee et al. [87], Iannelli e Denaro [76], Guy e Fogelson [65] apresentaram resultados e discussões sobre o tratamento das condições de contorno em fronteiras rígidas. Entretanto, o uso de condições de contorno modificadas é uma estratégia delicada. Alguns dos esquemas citados anteriormente apresentaram bons resultados, mas todo cuidado deve ser tomado na implementação para que a segunda ordem de precisão temporal não seja destruída. O recente trabalho de Guermond et al. [61] apresenta detalhes de implementação e comentários sobre várias extensões dos métodos de projeção. Em [61], os autores resumiram em uma tabela os diversos esquemas apresentados na literatura, descrevendo a forma do tratamento das condições de contorno, a estabilidade e ordem de precisão dos respectivos esquemas. Outro trabalho que descreve cuidadosamente os detalhes nas condições de contorno é o de Brown et al. [13]. 
Uma forma interessante de análise de métodos de projeção de segunda ordem evitando os problemas com as condições de contorno é por meio do "método de fatoração aproximada" [176]. Neste método, o desacoplamento das equações de Navier-Stokes (2.1) e (2.2) pode ser visto como uma fatoração aproximada das equações na forma discreta. Para os pesquisadores que utilizam esta estratégia, a principal vantagem do método de fatoração aproximada é que o desacoplamento e a preservação da precisão temporal estão associados com a fatoração aproximada do sistema das equações (2.1) e (2.2), e não com as condições de contorno ou a forma de atualização de pressão. Portanto, no estudo da ordem de precisão temporal dos métodos de projeção, erros devido às condições de contorno podem ser desconsiderados. Ou seja, quando utiliza-se o método de fatoração aproximada, as condições de contorno para as quantidades nãofísicas não são requeridas, pois essas informações já estão incorporadas nas matrizes e vetores da fatoração aproximada.

O método da fatoração aproximada para as equações de Navier-Stokes foi inicialmente estudado por Dukowicz e Dvinsky [38], que apresentaram um método de projeção de segunda ordem de precisão temporal. Logo após, Perot [125] apresentou um estudo do método de fatoração aproximada utilizando a conhecida decomposição LU. De acordo com Perot, a construção de um método de projeção de alta ordem via fatoração do tipo LU pode superar algumas dificuldades encontradas em uma análise padrão. Essa afirmação baseia-se no fato de que o erro introduzido no momento do desacoplamento das equações (2.1) e (2.2) está diretamente conectado à fatoração aproximada utilizada, e não com a correção da pressão e nem com as condições de contorno. Em [125], Perot apresenta alternativas na construção de um método de projeção de ordem dois.

Os trabalhos de Dukowicz e Dvinsky [38] e Perot [125] despertaram a atenção de outros pesquisadores que começaram a analisar o método de fatoração aproximada. Quarteroni et al. [134] apresentaram um estudo de fatorações em blocos de sistemas algébricos para a aproximação numérica das equações de Navier-Stokes. Lee et al. [87] apresentaram um estudo detalhado de fatorações aproximadas e exatas para a construção de métodos de projeção de alta ordem. Em [87] os autores destacaram, principalmente, as várias formas de matrizes resultantes da decomposição LU. Chang et al. [19] analisaram um método de passo fracionário utilizando a estratégia da fatoração aproximada. Recentemente, Henriksen e Holmen [67], Ni et al. [108], Zhang [181], entre outros, também apresentaram métodos de projeção de segunda ordem de precisão temporal obtidos e analisados pela fatoração aproximada.

Enfim, como vários autores têm apresentado diversas estratégias e análises na construção de métodos de alta ordem de precisão temporal, muitos trabalhos mostram comparações entre 
diversos esquemas propostos na literatura. Em particular, em [13, 19, 7, 6, 76, 35, 94, 65, 61] exemplos desse tipo de exercício podem ser verificados.

Como mencionado nos capítulos anteriores, de acordo com Guermond et al. [61] e Yang e Prosperetti [177], poucos trabalhos foram apresentados sobre métodos de projeção para escoamento de fluidos incompressíveis com superfície livre. Por exemplo, no contexto dos métodos de Volume-de-Fluido, Pilliod e Puckett [128] e Lorstad e Fuchs [97] apresentaram esquemas de segunda ordem no tempo e no espaço para tratamento de problemas com superfície livre. Em particular, Lorstad e Fuchs [97] analisaram a ordem do seu esquema na simulação numérica de escoamentos de bolhas. Para escoamentos multifásicos com superfície livre, Chen et al. [20] descrevem um algoritmo, baseado em elementos finitos, com ordem de precisão igual a dois. Recentemente, Yang e Prosperetti [177] apresentaram um método de projeção de segunda ordem para escoamentos com superfície livre em aplicações com contorno ajustado (boundary-fitted problem).

Entretanto, no contexto do método MAC para escoamentos com superfície livre, usando diferenças finitas e malhas deslocadas, ainda existem vários problemas em aberto, e poucos resultados foram apresentados até o presente momento. Uma possível justificativa para esse fato, é que problemas com superfícies livres adicionam outras dificuldades, como por exemplo, a influência na precisão temporal das condições de contorno da superfície livre. Após o trabalho pioneiro de Harlow e Welch [66], que propôs o método MAC, vários autores se dedicaram à extensão de métodos de projeção para problemas com superfície livre. Em 1994, Tomé e McKee [163] apresentaram uma versão melhorada do método MAC, o método GENSMAC. Porém, esse método utiliza uma formulação explícita, e portanto baseia-se num método de projeção de primeira ordem. Com o objetivo de aumentar a precisão temporal do método de Tomé e Mckee [163], no presente trabalho foi apresentado modificações no método de projeção implementado no método GENSMAC. Os resultados obtidos, como já descritos anteriormente, foram satisfatório do ponto de vista numérico, entretanto, não foi feita uma verificação da precisão temporal do método de projeção utilizado.

Portanto, no contexto do método MAC, parece que, um estudo da precisão temporal dos métodos de projeção para escoamento com superfície livre e baixo número de Reynolds ainda não foi apresentado na literatura. Desta forma, a principal motivação deste capítulo, é descrever métodos de projeção de ordem dois para problemas com superfícies livres. 


\subsection{Método de projeção de ordem dois para a formulação $\mathrm{SI}-\mathrm{AB} / \mathrm{CN}$}

A formulação semi-implícita com o método de Crank-Nicolson (SI/CN) foi apresentada no capítulo 4, seção 4.3, e sua extensão para o caso tridimensional no capítulo 5, seção 5.2. Os resultados numéricos foram muito satisfatórios, como pôde ser visto nas seções de resultados numéricos dos capítulos 4 e 5 , e isto motivou o estudo de como obter segunda ordem de precisão temporal nesta formulação.

Inicialmente, para construir um método semi-implícito estável, consistente e agora de ordem dois, considere a discretização temporal para as equações de Navier-Stokes, baseada em uma discretização de ordem dois, aplicando os parâmetros da tabela 2.1, apresentada no capítulo 2, como $\theta_{1}=\theta_{3}=\theta_{4}=0.5, \theta_{2}=1.5$ e $\alpha=0.5$ na equação (2.17), ou seja

$\frac{\mathbf{u}^{(n+1)}}{\delta t}-\frac{1}{2 R e} \nabla^{2} \mathbf{u}^{(n+1)}=\frac{\mathbf{u}^{(n)}}{\delta t}+\frac{1}{2 R e} \nabla^{2} \mathbf{u}^{(n)}-\frac{3}{2} \nabla \cdot(\mathbf{u u})^{(n)}+\frac{1}{2} \nabla \cdot(\mathbf{u u})^{(n-1)}-\nabla p^{\left(n+\frac{1}{2}\right)}+\left(F r^{2}\right)^{-1} \mathbf{g}^{(n)}$,

onde $\nabla p^{\left(n+\frac{1}{2}\right)}=\frac{\nabla p^{(n+1)}+\nabla p^{(n)}}{2}$, e considere também a equação da continuidade

$$
\nabla \cdot \mathbf{u}^{(n+1)}=0
$$

Note que agora, a formulação semi-implícita combina o esquema de Adams-Bashforth aplicado aos termos convectivos, com o método de Crank-Nicolson para os termos difusivos, e portanto, será denotada como SI-AB/CN.

De acordo com a estratégia dos métodos de projeção, baseado nesta equação, pode-se construir uma equação para o cálculo da velocidade intermediária $\widetilde{\mathbf{u}}$ como

$$
\frac{\widetilde{\mathbf{u}}}{\delta t}-\frac{1}{2 R e} \nabla^{2} \widetilde{\mathbf{u}}=\frac{\mathbf{u}^{(n)}}{\delta t}+\frac{1}{2 R e} \nabla^{2} \mathbf{u}^{(n)}-\frac{3}{2} \nabla \cdot(\mathbf{u u})^{(n)}+\frac{1}{2} \nabla \cdot(\mathbf{u u})^{(n-1)}-\nabla p^{\left(n-\frac{1}{2}\right)}+\left(F r^{2}\right)^{-1} \mathbf{g}^{(n)},
$$

onde $p^{\left(n-\frac{1}{2}\right)}$ é uma aproximação de $p^{\left(n+\frac{1}{2}\right)}$.

Utilizando o mesmo procedimento da seção 4.3, substituindo $\widetilde{\mathbf{u}}$, dado pela equação

$$
\mathbf{u}^{(n+1)}=\widetilde{\mathbf{u}}-\delta t \nabla \psi^{(n+1)}
$$


na equação (6.2), e comparando o resultado com a equação (6.1) obtém-se a fórmula de atualização da pressão como

$$
p^{\left(n+\frac{1}{2}\right)}=p^{\left(n-\frac{1}{2}\right)}+\psi^{(n+1)}-\frac{\delta t}{2 R e} \nabla^{2} \psi^{(n+1)} .
$$

Seguindo a estratégia para construir um método estável em escoamento com superfície livre bidimensional, deve-se discretizar implicitamente a equação da pressão na superfície livre (2.11), mas agora no nível de tempo $\left(n+\frac{1}{2}\right)$ como

$$
-p^{\left(n+\frac{1}{2}\right)}+\frac{2}{R e}\left[\frac{\partial u}{\partial x} n_{x}^{2}+\frac{\partial v}{\partial y} n_{y}^{2}+\left(\frac{\partial u}{\partial y}+\frac{\partial v}{\partial x}\right) n_{x} n_{y}\right]^{\left(n+\frac{1}{2}\right)}=0 \quad \text { em } \quad \partial \Omega_{4} .
$$

A estratégia para desacoplar os campos de velocidade e pressão da equação acima é a mesma descrita no capítulo 4, seção 4.3. A principal diferença é que agora utiliza-se a discretização no nível de tempo $\left(n+\frac{1}{2}\right)$, e isto determinará uma média entre as velocidades no nível de tempo $(n+1)$ e $(n)$, fato que não ocorria nas formulações anteriores.

Novamente, considere primeiro o caso onde uma célula $[\mathbf{S}]$ da superfície livre está em contato com uma célula $[\mathbf{E}]$ como apresentado anteriormente na figura 2.4a). Neste caso o vetor normal é $\mathbf{n}=(1,0)$ e a equação (6.4), após a utilização da equação da continuidade discretizada no nível de tempo $\left(n+\frac{1}{2}\right)$, reduz a

$$
p^{\left(n+\frac{1}{2}\right)}=\frac{2}{R e}\left(\frac{\partial u^{\left(n+\frac{1}{2}\right)}}{\partial x}\right)=-\frac{2}{R e}\left(\frac{\partial v^{\left(n+\frac{1}{2}\right)}}{\partial y}\right)=-\frac{1}{R e}\left(\frac{\partial\left(v^{(n+1)}+v^{(n)}\right)}{\partial y}\right) .
$$

Substituindo as equações (4.7) e (6.3) em (6.5), e reagrupando os termos obtém-se

$$
\psi^{(n+1)}-\frac{\delta t}{2 R e} \nabla^{2} \psi^{(n+1)}-\frac{\delta t}{R e}\left(\frac{\partial^{2} \psi^{(n+1)}}{\partial y^{2}}\right)=-\frac{1}{R e}\left(\frac{\partial \widetilde{v}}{\partial y}+\frac{\partial v^{(n)}}{\partial y}\right)-p^{\left(n-\frac{1}{2}\right)} .
$$

Desta forma, a equação (6.6) é usada para definir $\psi$ na superfície livre para a configuração da figura 2.4a).

O segundo caso é dado pela configuração apresentada anteriormente na figura 2.6a) onde $\mathbf{n}=\left(\frac{\sqrt{2}}{2}, \frac{\sqrt{2}}{2}\right)$, reescrevendo a equação $(6.4)$ como

$$
p^{\left(n+\frac{1}{2}\right)}=\frac{1}{R e}\left(\frac{\partial u^{\left(n+\frac{1}{2}\right)}}{\partial y}+\frac{\partial v^{\left(n+\frac{1}{2}\right)}}{\partial x}\right)=\frac{1}{2 R e}\left(\frac{\partial u^{(n+1)}}{\partial y}+\frac{\partial v^{(n+1)}}{\partial x}+\frac{\partial u^{(n)}}{\partial y}+\frac{\partial v^{(n)}}{\partial x}\right) .
$$


Após substituir as equações da atualização da velocidade (4.7) e (4.11), e a fórmula da correção da pressão (6.3) em (6.7) tem-se

$$
\psi^{(n+1)}-\frac{\delta t}{2 R e} \nabla^{2} \psi^{(n+1)}+\frac{\delta t}{R e}\left(\frac{\partial^{2} \psi^{(n+1)}}{\partial x \partial y}\right)=\frac{1}{2 R e}\left(\frac{\partial \widetilde{u}}{\partial y}+\frac{\partial \widetilde{v}}{\partial x}+\frac{\partial u^{(n)}}{\partial y}+\frac{\partial v^{(n)}}{\partial x}\right)-p^{\left(n-\frac{1}{2}\right)},
$$

e portanto, esta equação é utilizada para definir $\psi$ na superfície livre para a configuração da figura 2.6a).

Seguindo o procedimento acima, a construção da equação para $\psi$ nos casos descritos nas figuras 2.4b) e 2.5, e para os outros casos ilustrados na figura 2.6, é muito semelhante, e não será apresentada neste trabalho.

O algoritmo utilizado neste método de ordem dois apresenta algumas pequenas modificações daquele apresentado anteriormente para a formulação SI-CN no capítulo 4, seção 4.3, subseção 4.3.1.

Uma diferença fundamental ocorre no passo 1 para o cálculo da velocidade intermediária u. Agora, além de utilizar a equação de quantidade de movimento (6.2), deve-se aplicar uma extrapolação linear no tempo para as condições de contorno de $\widetilde{\mathbf{u}}$ na superfície livre. Isto é, agora quando na solução da equação (6.2) requer o valor de $\widetilde{\mathbf{u}}$ na superfície livre, ao invés de usar $\widetilde{\mathbf{u}}=\mathbf{u}^{(n)}$ como anteriormente (veja o algoritmo da subseção 4.3.1), usa-se

$$
\widetilde{\mathbf{u}}=2 \mathbf{u}^{(n)}-\mathbf{u}^{(n-1)}
$$

onde $\mathbf{u}^{(n)}$ é calculado das condições $(2.2)$ e (2.12).

No passo 2, a idéia é a mesma do algoritmo anterior da formulação SI-CN: utilizar a equação de Poisson

$$
\nabla^{2} \psi^{(n+1)}=\frac{1}{\delta t} \nabla \cdot \widetilde{\mathbf{u}}
$$

junto com as novas equações derivadas para a pressão tentativa $\psi^{(n+1)}$ na superfície livre descritas nesta seção (veja as equações (6.6) e (6.8)).

No passo 4 aplica-se agora a equação (6.3) no lugar de (4.17).

Os passos 3 e 5 são análogos aos do algoritmo da formulação SI-CN (veja subseção 4.3.1).

Portanto, as principais diferenças desta formulação SI-AB/CN para a anterior podem ser resumidas em três pontos: i) uso da equação de quantidade de movimento (6.2), ii) discretização implícita da equação da pressão na superfície livre no nível de tempo $\left(n+\frac{1}{2}\right)$ e iii) aplicação 
de uma extrapolação linear no tempo para $\widetilde{\mathbf{u}}$ na superfície livre na solução da equação de quantidade de movimento.

\subsection{Método de projeção de ordem dois para a formulação laplaciano superficial}

Nesta seção propõem-se um novo tratamento das condições de contorno da pressão derivando um novo método de projeção de ordem dois para escoamentos com superfície livre a baixo número de Reynolds. Os métodos descritos até o momento nesta tese alcançaram ótimos resultados de estabilidade em escoamentos com superfície livre. Entretanto, estas formulações envolvem a solução de um extenso sistema linear não-simétrico, devido à discretização implícita da equação da pressão na superfície livre, e isto acarreta um aumento no tempo de processamento. Desta forma, nesta seção apresenta-se, idéias iniciais na construção de uma formulação que envolve a solução do laplaciano superficial. Baseado nesta formulação, descreve-se como obter um método de ordem dois, estável, e que utilize apenas a solução de sistemas lineares simétricos em todo domínio e no contorno.

A construção desta estratégia inicia utilizando as mesmas técnicas descritas na seção anterior, ou seja, definindo a equação para o cálculo do campo intermediário (6.2). Entretanto, nesta formulação, utiliza-se a fórmula simples para a correção da pressão, descrita anteriormente no capítulo 2 pela equação $(2.32)$, no nível de tempo $\left(n+\frac{1}{2}\right)$, isto é

$$
p^{\left(n+\frac{1}{2}\right)}=p^{\left(n-\frac{1}{2}\right)}+\psi^{(n+1)} .
$$

A estratégia de discretizar a equação da pressão na superfície livre implicitamente para obter um método estável também é utilizada, e resultará na formulação do laplaciano superficial. Para isto, inicialmente, utiliza-se alguns conceitos básicos de geometria diferencial. Admite-se que a superfície livre é uma curva $\omega$ parametrizada pelo comprimento de arco (ver detalhes em [153]) como ilustrada na figura 6.1.

Considere

$$
\begin{aligned}
& \mathbf{n}(s)=(-\dot{y}(s), \dot{x}(s))=\left(n_{x}(s), n_{y}(s)\right), \\
& \mathbf{m}(s)=(\dot{x}(s), \dot{y}(s))=\left(m_{x}(s), m_{y}(s)\right),
\end{aligned}
$$

os vetores normal e tangencial à curva $\omega$, respectivamente. Desta forma $\mathbf{m}(s)=\dot{\omega}(s)$. 


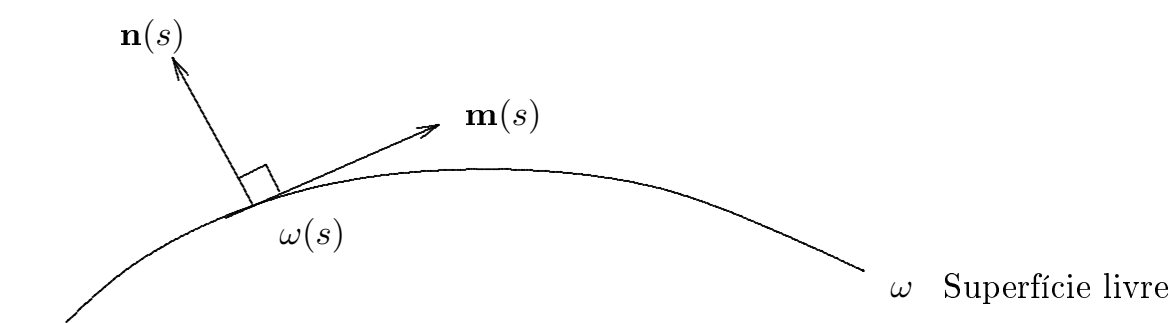

Fluido

Figura 6.1: Ilustração dos vetores normal $(\mathbf{n}(s))$ e tangencial $(\mathbf{m}(s))$ em uma curva $\omega$ parametrizada pelo comprimento de arco.

A derivada do vetor tangente em (6.11) é

$$
\dot{\mathbf{m}}(s)=(\ddot{x}(s), \ddot{y}(s))=\left(\dot{m}_{x}(s), \dot{m}_{y}(s)\right) .
$$

Para cada $s \in I$, tem-se que $\{\mathbf{m}(s), \mathbf{n}(s)\}$ é uma base ortonormal do $\mathbb{R}^{2}$, onde $I$ é um intervalo. De acordo com a primeira equação de Frenet [153], a curvatura $\kappa(s)$ de $\omega$ pode ser calculada por

$$
\dot{\mathbf{m}}(s)=\kappa(s) \mathbf{n}(s),
$$

ou seja

$$
\begin{aligned}
& \dot{m}_{x}(s)=\kappa n_{x}(s), \\
& \dot{m}_{y}(s)=\kappa n_{y}(s) .
\end{aligned}
$$

A velocidade normal $u_{n}$ a curva $\omega$ em $s$ é dada por

$$
u_{n}(s)=u n_{x}(s)+v n_{y}(s),
$$

enquanto que, a velocidade tangencial

$$
u_{m}(s)=u m_{x}(s)+v m_{y}(s) .
$$

Definindo-se a derivada normal de $u_{n}$ como

$$
\begin{aligned}
& \frac{\partial u_{n}(x(s), y(s))}{\partial n}=\lim _{\varepsilon \rightarrow 0} \frac{\mathbf{u}(\mathbf{x}+\varepsilon \mathbf{n}(\mathbf{x})) \cdot \mathbf{n}(\mathbf{x})-\mathbf{u}(\mathbf{x}) \cdot \mathbf{n}(\mathbf{x})}{\varepsilon} \\
& =\lim _{\varepsilon \rightarrow 0} \frac{u\left(x+\varepsilon n_{x}, y+\varepsilon n_{y}\right) n_{x}+v\left(x+\varepsilon n_{x}, y+\varepsilon n_{y}\right) n_{y}-u(x, y) n_{x}-v(x, y) n_{y}}{\varepsilon} .
\end{aligned}
$$


Utilizando-se a fórmula de Taylor para os termos $u\left(x+\varepsilon n_{x}, y+\varepsilon n_{y}\right)$ e $v\left(x+\varepsilon n_{x}, y+\varepsilon n_{y}\right)$ na equação (6.17), obtém-se

$$
\frac{\partial u_{n}(x(s), y(s))}{\partial n}=\frac{\partial u}{\partial x} n_{x}^{2}+\frac{\partial v}{\partial x} n_{x} n_{y}+\frac{\partial u}{\partial y} n_{x} n_{y}+\frac{\partial v}{\partial y} n_{y}^{2}
$$

De acordo com a definição de derivada direcional, tem-se

$$
\begin{aligned}
& \frac{d u_{m}(s)}{d s}=\frac{d\left[u m_{x}(s)+v m_{y}(s)\right]}{d s}=\frac{d\left[u(x(s), y(s)) m_{x}(s)+v(x(s), y(s)) m_{y}(s)\right]}{d s} \\
& =\left(\frac{\partial u}{\partial x} \dot{x}(s)+\frac{\partial u}{\partial y} \dot{y}(s)\right) m_{x}(s)+u \dot{m}_{x}(s)+\left(\frac{\partial v}{\partial x} \dot{x}(s)+\frac{\partial v}{\partial y} \dot{y}(s)\right) m_{y}(s)+v \dot{m}_{y}(s) .
\end{aligned}
$$

Substituindo as equações (6.11) e (6.14) em (6.19) obtém-se

$$
\frac{d u_{m}(s)}{d s}=\frac{\partial u}{\partial x} m_{x}^{2}+\frac{\partial v}{\partial x} m_{x} m_{y}+\frac{\partial u}{\partial y} m_{x} m_{y}+\frac{\partial v}{\partial y} m_{y}^{2}+\kappa\left(u n_{x}+v n_{y}\right) .
$$

Ao somar as derivadas em (6.18) e (6.20), e utilizando a propriedade de ortogonalidade entre $\mathbf{n}(s)$ e $\mathbf{m}(s)$, tem-se

$$
\frac{\partial u_{n}}{\partial n}+\frac{d u_{m}}{d s}-\kappa u_{n}=\frac{\partial u}{\partial x}+\frac{\partial v}{\partial y}=\nabla \cdot \mathbf{u}
$$

Em particular, para escoamentos incompressíveis, a equação (6.21) reduz-se

$$
\frac{\partial u_{n}}{\partial n}+\frac{d u_{m}}{d s}-\kappa u_{n}=0
$$

Quando a curva $\omega$ é côncava em relação a atmosfera (como na figura 6.1) assume-se que a curvatura é negativa, caso contrário, será positiva.

Note que, substituindo o lado direito de (6.18) na equação da pressão na superfície livre (2.11), pode-se reescrever a condição de tensão normal na forma

$$
-p+\frac{2}{R e}\left(\frac{\partial u_{n}}{\partial n}\right)=0
$$

com $p=p(x(s), y(s))$. Substituindo (6.22) em (6.23) obtém-se

$$
p+\frac{2}{R e}\left(\frac{d u_{m}}{d s}-\kappa u_{n}\right)=0
$$


Portanto, nesta formulação, a condição de tensão normal (2.11) é substituída pela equação (6.23) que pode ser reescrita na forma da equação (6.24).

O próximo passo será a construção do laplaciano superficial. Para isto, de acordo com a equação (2.27), defini-se a velocidade final $u_{m}$ como

$$
u_{m}^{(n+1)}=\widetilde{u}_{m}-\delta t \frac{d \psi_{s}^{(n+1)}}{d s}
$$

onde $\psi_{s}=\psi(x(s), y(s))$.

Discretizando implicitamente a equação (6.24) no nível de tempo $\left(n+\frac{1}{2}\right)$ tem-se

$$
p^{\left(n+\frac{1}{2}\right)}=-\frac{2}{R e}\left(\frac{d u_{m}}{d s}-\kappa u_{n}\right)^{\left(n+\frac{1}{2}\right)}=-\frac{1}{R e}\left(\frac{d\left(u_{m}^{(n+1)}+u_{m}^{(n)}\right)}{d s}-\kappa\left(u_{n}^{(n+1)}+u_{n}^{(n)}\right)\right) .
$$

Substituindo as equações (6.10) e (6.25) em (6.26) tem-se

$$
\psi_{s}^{(n+1)}-\frac{\delta t}{R e}\left(\frac{d^{2} \psi_{s}^{(n+1)}}{d s^{2}}\right)-\frac{\kappa}{R e} u_{n}^{(n+1)}=-\frac{1}{R e}\left(\frac{d \widetilde{u}_{m}}{d s}+\frac{d u_{m}^{(n)}}{d s}-\kappa u_{n}^{(n)}\right)-p^{\left(n-\frac{1}{2}\right)},
$$

na qual o termo $\frac{d^{2} \psi_{s}^{(n+1)}}{d s^{2}}$ recebe o nome de laplaciano superficial. Na equação (6.27) o termo do laplaciano superficial $\frac{d^{2} \psi^{(n+1)}}{d s^{2}}$ pode ser discretizado utilizando apenas três pontos na superfície livre (ou seja, apenas células vizinhas do tipo [S]), resultando em um sistema linear simétrico e tridiagonal, e esta é a principal vantagem desta formulação em relação as anteriores.

Note que, na equação (6.27) ainda há um acoplamento entre $\psi_{s}$ e $u_{n}$ no nível de tempo $(n+1)$, e portanto é necessário o desenvolvimento de uma estratégia para o desacoplamento. Entretanto, como o objetivo principal desta seção é apenas iniciar os estudos da técnica do laplaciano superficial, evita-se este acoplamento simplificando a equação (6.27). Para isto, assumi-se que esta equação é aplicada em um escoamento com superfície livre planar $(\kappa=0)$ ou um escoamento estacionário $\left(u_{n}=0\right)$. Logo, a equação (6.27) é reescrita como

$$
\psi_{s}^{(n+1)}-\frac{\delta t}{R e}\left(\frac{d^{2} \psi_{s}^{(n+1)}}{d s^{2}}\right)=-\frac{1}{R e}\left(\frac{d \widetilde{u}_{m}}{d s}+\frac{d u_{m}^{(n)}}{d s}\right)-p^{\left(n-\frac{1}{2}\right)} .
$$

As equações de diferenças finitas para este caso estão dadas no Apêndice A.

O algoritmo utilizado nesta formulação apresenta algumas pequenas modificações em relação ao descrito na seção anterior. 
Uma estratégia comum utilizada no passo 1 para obter ordem dois é aplicar a extrapolação linear no tempo (6.9) para a condição de contorno na superfície livre na velocidade intermediária $\widetilde{\mathbf{u}}$, quando resolve-se a equação de quantidade de movimento (6.2).

O passo 2 desta estratégia é modificado, sendo dividido em duas partes. Inicialmente, como o valor de $\psi^{(n+1)}$ na superfície livre é calculado apenas por um tipo de equação, que sempre utiliza apenas células do tipo [S], utiliza-se a equação (6.28). Após obter o valor de $\psi$ em todas as células de superfície livre, calcula-se a equação de Poisson

$$
\nabla^{2} \psi^{(n+1)}=\frac{1}{\delta t} \nabla \cdot \widetilde{\mathbf{u}}
$$

As condições de contorno para $\psi$ nos domínios de parede rígida, injetor e ejetor utilizadas na solução das equações neste passo foram apresentadas no algoritmo da subseção 4.3.1, seção 4.3.

No passo 4 aplica-se a equação (6.10). Os passos 3 e 5 são análogos aos do algoritmo da formulação SI-CN (veja subseção 4.3.1).

Para obter a ordem dois, as mesma estratégias descritas na formulação da seção anterior devem ser aplicadas na formulação do laplaciano superficial.

\subsection{Verificação}

As formulações apresentadas nesta seção foram verificadas para um problema teste. Considerou-se as equações de Navier-Stokes (2.1) e (2.2) aplicadas em um domínio $\Omega=\left(-\frac{\pi}{2},-\frac{\pi}{2}\right) \times$ $\left(\frac{\pi}{2}, \frac{\pi}{2}\right)$. As condições de contorno impostas para este problema foram de superfície livre, isto é, utilizou-se as equações (2.11) e (2.12) nas laterais, e nas partes superior e inferior do domínio. Desta forma, a configuração deste problema foi escolhida especificamente para testar os métodos de projeção deste capítulo, pois apenas condições de contorno de superfície livre planar são aplicadas em $\partial \Omega_{4}$.

O passo espacial usado foi $\delta x=\delta y=0.157 m(20 \times 20$ células $)$ e o coeficiente de viscosidade $\nu=10.0 \mathrm{~m}^{2} \mathrm{~s}^{-1}$, com velocidade de escala $U=1.0 \mathrm{~ms}^{-1}$ e o comprimento de escala $L=1.0 \mathrm{~m}$ resultando em $R e=0.1$. O escoamento foi integrado de $t=0 \mathrm{~s}$ até $t=0.1 \mathrm{~s}$. Em $t=0 \mathrm{~s}$, assumiu-se que os campos de velocidade e pressão são iguais a zero em todo o domínio e nos contornos $\partial \Omega_{4}$. Após $t>0.02 s$, aplicou-se uma força superficial $f_{s}(t)=0.25(1-\cos (2 \pi(t-$ 0.02))) na equação da pressão na superfície livre (2.11) para gerar os campos de velocidade e pressão. Esta força pode ser interpretada como um termo forçante para a equação (2.11) 
quando $\mathbf{n}=(1,0)$, ou seja, $f_{s}(t)$ é aplicada apenas nos contornos de superfície livre que são aproximados quando o vetor normal está apontado na direção horizontal positiva (em $x=\frac{\pi}{2} \mathrm{e}$ $\left.-\frac{\pi}{2}<y<0\right)$.

As soluções de referência para as velocidades e pressão neste problema foram calculadas com $\delta t=1.0 \times 10^{-5} s$. Os erros foram calculados comparando a solução de referência com as soluções em $\delta t_{1}=2.0 \times 10^{-3} s, \delta t_{2}=0.5 \delta t_{1}$ e $\delta t_{3}=0.5 \delta t_{2}$. As comparações foram realizadas para todos os pontos do domínio e dos contornos em $t=0.06 \mathrm{~s}$ utilizando os erros absolutos nas normas $l_{2}, l_{1}$ e $l_{\infty}$. As tabelas 6.1-6.4 apresentam os resultados para a formulação SI-AB/CN descrita na seção 6.2, e as tabelas 6.5-6.8 para a formulação laplaciano superficial descrita na seção anterior.

Os resultados apresentados nas tabelas 6.1 e 6.5 confirmam que os esquemas apresentados neste capítulo são de ordem dois. Quando estas formulações são aplicadas, porém sem a extrapolação linear (6.9) para as condições de contorno de $\widetilde{\mathbf{u}}$ na superfície livre na solução da equação de quantidade de movimento (6.2), a ordem do método diminui (veja por exemplo a tabela 6.2 para a formulação SI-AB/CN, e a tabela 6.6 para o laplaciano superficial.)

As tabelas 6.3 e 6.7 mostram os resultados das formulações com a extrapolação linear (6.9), entretanto aplicando a discretização implícita da equação da pressão na superfície livre no nível de tempo $(n+1)$, como nas formulações do capítulo 4 . Ou seja, na primeira formulação aplicou-se a equação (4.3) ao invés de (6.4), enquanto que na formulação laplaciano superficial discretiza-se a equação (6.24) no nível de tempo $(n+1)$, e não como em (6.26) . Os resultados das tabelas 6.3 e 6.7 evidenciam que a aplicação da discretização no nível de tempo $(n+1)$ da equação da pressão na superfície livre resulta em formulações que não atingem ordem dois. Em particular, a formulação SI-AB/CN neste caso apresentou melhores resultados.

Finalmente, mostrou-se com os resultados das tabelas 6.4 e 6.8, que as formulações, respectivamente, SI-AB/CN e laplaciano superficial sem a extrapolação linear (6.9) e sem a discretização da equação da pressão na superfície livre no nível de tempo $\left(n+\frac{1}{2}\right)$ são apenas de ordem um.

\subsection{Considerações finais}

Neste capítulo foram apresentadas estratégias para obter métodos de projeção de ordem dois em escoamentos com superfície livre a baixo número de Reynolds. Duas formulações foram derivadas. A primeira é uma extensão da formulação apresentada na seção 4.3, utilizando uma 
Tabela 6.1: Erros para a formulação SI-AB/CN da seção 6.2. $\left(R e=0.1, \delta t_{1}=2.0 \times 10^{-3} s\right.$, $\delta t_{2}=1.0 \times 10^{-3} s$ e $\left.\delta t_{3}=5.0 \times 10^{-4} s\right)$.

\begin{tabular}{|c|c|c|c|c|c|c|}
\hline & $\delta t_{1}$ & $N_{1}$ & $\delta t_{2}$ & $N_{2}$ & $\delta t_{3}$ & $N_{m} \simeq$ \\
\hline velocidade- $u$ & & & & & & \\
$l_{2}$ & $2.090 \times 10^{-5}$ & 1.92 & $5.492 \times 10^{-6}$ & 1.97 & $1.399 \times 10^{-6}$ & 1.94 \\
$l_{1}$ & $2.222 \times 10^{-4}$ & 1.91 & $5.876 \times 10^{-5}$ & 1.96 & $1.500 \times 10^{-5}$ & 1.93 \\
$l_{\infty}$ & $4.321 \times 10^{-6}$ & 1.93 & $1.131 \times 10^{-6}$ & 1.97 & $2.876 \times 10^{-7}$ & 1.95 \\
velocidade- $v$ & & & & & & \\
$l_{2}$ & $9.412 \times 10^{-6}$ & 1.94 & $2.448 \times 10^{-6}$ & 1.97 & $6.214 \times 10^{-7}$ & 1.95 \\
$l_{1}$ & $1.129 \times 10^{-4}$ & 1.93 & $2.955 \times 10^{-5}$ & 1.97 & $7.520 \times 10^{-6}$ & 1.95 \\
$l_{\infty}$ & $2.492 \times 10^{-6}$ & 1.93 & $6.497 \times 10^{-7}$ & 1.97 & $1.649 \times 10^{-7}$ & 1.95 \\
pressão- $p$ & & & & & & \\
$l_{2}$ & $9.062 \times 10^{-4}$ & 1.92 & $2.394 \times 10^{-4}$ & 1.97 & $6.108 \times 10^{-5}$ & 1.94 \\
$l_{1}$ & $8.307 \times 10^{-3}$ & 1.93 & $2.179 \times 10^{-3}$ & 1.97 & $5.546 \times 10^{-4}$ & 1.95 \\
$l_{\infty}$ & $3.623 \times 10^{-4}$ & 1.88 & $9.819 \times 10^{-5}$ & 1.95 & $2.525 \times 10^{-5}$ & 1.91 \\
\hline
\end{tabular}

Tabela 6.2: Erros para a formulação SI-AB/CN da seção 6.2 sem a extrapolação linear no tempo (6.9) para as condições de contorno de $\widetilde{\mathbf{u}}$ na solução da equação (6.2). (Re = 0.1, $\delta t_{1}=2.0 \times 10^{-3} s, \delta t_{2}=1.0 \times 10^{-3} s$ e $\left.\delta t_{3}=5.0 \times 10^{-4} s\right)$.

\begin{tabular}{|c|c|c|c|c|c|c|}
\hline & $\delta t_{1}$ & $N_{1}$ & $\delta t_{2}$ & $N_{2}$ & $\delta t_{3}$ & $N_{m} \simeq$ \\
\hline velocidade- $u$ & & & & & & \\
$l_{2}$ & $6.706 \times 10^{-5}$ & 1.02 & $3.302 \times 10^{-5}$ & 1.02 & $1.626 \times 10^{-5}$ & 1.02 \\
$l_{1}$ & $5.929 \times 10^{-4}$ & 1.06 & $2.832 \times 10^{-4}$ & 1.04 & $1.370 \times 10^{-4}$ & 1.05 \\
$l_{\infty}$ & $1.616 \times 10^{-5}$ & 0.94 & $8.410 \times 10^{-6}$ & 0.97 & $4.267 \times 10^{-6}$ & 0.95 \\
velocidade- $v$ & & & & & & \\
$l_{2}$ & $4.211 \times 10^{-5}$ & 0.92 & $2.214 \times 10^{-5}$ & 0.96 & $1.132 \times 10^{-5}$ & 0.95 \\
$l_{1}$ & $3.470 \times 10^{-4}$ & 1.02 & $1.705 \times 10^{-4}$ & 1.01 & $8.456 \times 10^{-5}$ & 1.01 \\
$l_{\infty}$ & $1.204 \times 10^{-5}$ & 0.93 & $6.285 \times 10^{-6}$ & 0.96 & $3.219 \times 10^{-6}$ & 0.93 \\
pressão- $p$ & & & & & & \\
$l_{2}$ & $1.196 \times 10^{-3}$ & 1.31 & $4.826 \times 10^{-4}$ & 1.10 & $2.242 \times 10^{-4}$ & 1.20 \\
$l_{1}$ & $1.230 \times 10^{-2}$ & 1.26 & $5.119 \times 10^{-3}$ & 1.08 & $2.410 \times 10^{-3}$ & 1.21 \\
$l_{\infty}$ & $3.328 \times 10^{-4}$ & 1.25 & $1.393 \times 10^{-4}$ & 1.22 & $5.976 \times 10^{-5}$ & 1.23 \\
\hline
\end{tabular}

estratégia semi-implícita com o método de Adams-Bashforth/Crank-Nicolson. A segunda usa uma equação para a pressão na superfície livre derivada do laplaciano superficial.

Os resultados numéricos para um problema teste mostraram que as formulações são de ordem dois para o campo de velocidade no nível de tempo $(n+1)$ e para a pressão no nível de tempo $\left(n+\frac{1}{2}\right)$. Duas estratégias foram fundamentais para obter esta ordem: uma extrapola- 
Tabela 6.3: Erros para a formulação SI-AB/CN da seção 6.2 com a equação da pressão na superfície livre discretizada no nível de tempo $(n+1)$. $\left(R e=0.1, \delta t_{1}=2.0 \times 10^{-3} s, \delta t_{2}=\right.$ $1.0 \times 10^{-3} s$ e $\left.\delta t_{3}=5.0 \times 10^{-4} s\right)$.

\begin{tabular}{|c|c|c|c|c|c|c|}
\hline & $\delta t_{1}$ & $N_{1}$ & $\delta t_{2}$ & $N_{2}$ & $\delta t_{3}$ & $N_{m} \simeq$ \\
\hline $\begin{array}{c}\text { velocidade- } u \\
l_{2}\end{array}$ & $2.979 \times 10^{-5}$ & 1.55 & $1.017 \times 10^{-5}$ & 1.42 & $3.776 \times 10^{-6}$ & 1.48 \\
$l_{1}$ & $2.819 \times 10^{-4}$ & 1.64 & $9.010 \times 10^{-5}$ & 1.54 & $3.091 \times 10^{-5}$ & 1.59 \\
$l_{\infty}$ & $6.907 \times 10^{-6}$ & 1.48 & $2.474 \times 10^{-6}$ & 1.36 & $9.615 \times 10^{-7}$ & 1.42 \\
velocidade- $v$ & & & & & & \\
$l_{2}$ & $1.675 \times 10^{-5}$ & 1.39 & $6.376 \times 10^{-6}$ & 1.27 & $2.640 \times 10^{-6}$ & 1.33 \\
$l_{1}$ & $1.601 \times 10^{-4}$ & 1.53 & $5.540 \times 10^{-5}$ & 1.37 & $2.139 \times 10^{-5}$ & 1.45 \\
$l_{\infty}$ & $4.546 \times 10^{-6}$ & 1.41 & $1.710 \times 10^{-6}$ & 1.29 & $6.959 \times 10^{-7}$ & 1.35 \\
pressão- $p$ & & & & & & \\
$l_{2}$ & $1.019 \times 10^{-3}$ & 1.74 & $3.036 \times 10^{-4}$ & 1.63 & $9.784 \times 10^{-5}$ & 1.68 \\
$l_{1}$ & $7.892 \times 10^{-3}$ & 1.99 & $1.984 \times 10^{-3}$ & 1.74 & $5.907 \times 10^{-4}$ & 1.86 \\
$l_{\infty}$ & $4.294 \times 10^{-4}$ & 1.69 & $1.330 \times 10^{-4}$ & 1.63 & $4.272 \times 10^{-5}$ & 1.66 \\
\hline
\end{tabular}

Tabela 6.4: Erros para a formulação SI-AB/CN da seção 6.2 sem a extrapolação linear no tempo (6.9) e com a equação da pressão na superfície livre discretizada no nível de tempo $(n+1)$. $\left(R e=0.1, \delta t_{1}=2.0 \times 10^{-3} s, \delta t_{2}=1.0 \times 10^{-3} s\right.$ e $\left.\delta t_{3}=5.0 \times 10^{-4} s\right)$.

\begin{tabular}{|c|c|c|c|c|c|c|}
\hline & $\delta t_{1}$ & $N_{1}$ & $\delta t_{2}$ & $N_{2}$ & $\delta t_{3}$ & $N_{m} \simeq$ \\
\hline velocidade- $u$ & & & & & & \\
$l_{2}$ & $7.401 \times 10^{-5}$ & 1.00 & $3.699 \times 10^{-5}$ & 1.01 & $1.837 \times 10^{-5}$ & 1.00 \\
$l_{1}$ & $6.383 \times 10^{-4}$ & 1.04 & $3.094 \times 10^{-4}$ & 1.03 & $1.511 \times 10^{-4}$ & 1.03 \\
$l_{\infty}$ & $1.833 \times 10^{-5}$ & 0.97 & $9.319 \times 10^{-6}$ & 0.99 & $4.666 \times 10^{-6}$ & 0.98 \\
velocidade- $v$ & & & & & & \\
$l_{2}$ & $4.792 \times 10^{-5}$ & 0.90 & $2.554 \times 10^{-5}$ & 0.95 & $1.315 \times 10^{-5}$ & 0.92 \\
$l_{1}$ & $3.833 \times 10^{-4}$ & 0.99 & $1.924 \times 10^{-4}$ & 0.99 & $9.674 \times 10^{-5}$ & 0.99 \\
$l_{\infty}$ & $1.366 \times 10^{-5}$ & 0.92 & $7.213 \times 10^{-6}$ & 0.96 & $3.689 \times 10^{-6}$ & 0.94 \\
pressão- $p$ & & & & & & \\
$l_{2}$ & $1.246 \times 10^{-3}$ & 1.37 & $4.824 \times 10^{-4}$ & 1.16 & $2.145 \times 10^{-4}$ & 1.26 \\
$l_{1}$ & $1.215 \times 10^{-2}$ & 1.30 & $4.912 \times 10^{-3}$ & 1.13 & $2.237 \times 10^{-3}$ & 1.21 \\
$l_{\infty}$ & $3.472 \times 10^{-4}$ & 1.24 & $1.466 \times 10^{-4}$ & 1.21 & $6.332 \times 10^{-5}$ & 1.22 \\
\hline
\end{tabular}

ção das condições de contorno de $\widetilde{\mathbf{u}}$ na solução da equação de quantidade de movimento e a discretização implícita no nível de tempo $\left(n+\frac{1}{2}\right)$ da equação da pressão na superfície livre.

Nesta seção, apenas a idéia inicial sobre a formulação do laplaciano superficial foi apresentada. Em particular, resolveu-se um problema simples com superfície livre planar. Neste caso, esta formulação resolve uma dificuldade encontrada nas formulações SI apresentadas nesta 
Tabela 6.5: Erros para a formulação laplaciano superficial da seção 6.3. $\left(R e=0.1, \delta t_{1}=\right.$ $2.0 \times 10^{-3} s, \delta t_{2}=1.0 \times 10^{-3} s$ e $\left.\delta t_{3}=5.0 \times 10^{-4} s\right)$.

\begin{tabular}{|c|c|c|c|c|c|c|}
\hline & $\delta t_{1}$ & $N_{1}$ & $\delta t_{2}$ & $N_{2}$ & $\delta t_{3}$ & $N_{m} \simeq$ \\
\hline velocidade- $u$ & & & & & & \\
$l_{2}$ & $4.770 \times 10^{-6}$ & 1.92 & $1.257 \times 10^{-6}$ & 1.97 & $3.199 \times 10^{-7}$ & 1.94 \\
$l_{1}$ & $3.478 \times 10^{-5}$ & 1.95 & $8.973 \times 10^{-6}$ & 1.98 & $2.272 \times 10^{-6}$ & 1.96 \\
$l_{\infty}$ & $2.337 \times 10^{-6}$ & 1.89 & $6.262 \times 10^{-7}$ & 1.96 & $1.598 \times 10^{-7}$ & 1.93 \\
velocidade- $v$ & & & & & & \\
$l_{2}$ & $4.788 \times 10^{-6}$ & 1.93 & $1.251 \times 10^{-6}$ & 1.97 & $3.176 \times 10^{-7}$ & 1.95 \\
$l_{1}$ & $3.027 \times 10^{-5}$ & 1.95 & $7.823 \times 10^{-6}$ & 1.98 & $1.981 \times 10^{-6}$ & 1.96 \\
$l_{\infty}$ & $2.056 \times 10^{-6}$ & 1.90 & $5.491 \times 10^{-7}$ & 1.97 & $1.400 \times 10^{-7}$ & 1.93 \\
pressão- $p$ & & & & & & \\
$l_{2}$ & $3.228 \times 10^{-4}$ & 1.92 & $8.485 \times 10^{-5}$ & 1.97 & $2.154 \times 10^{-5}$ & 1.95 \\
$l_{1}$ & $1.399 \times 10^{-3}$ & 1.92 & $3.695 \times 10^{-4}$ & 1.98 & $9.336 \times 10^{-5}$ & 1.95 \\
$l_{\infty}$ & $1.893 \times 10^{-4}$ & 1.91 & $5.013 \times 10^{-5}$ & 1.97 & $1.276 \times 10^{-5}$ & 1.94 \\
\hline
\end{tabular}

Tabela 6.6: Erros para a formulação laplaciano superficial da seção 6.3 sem a extrapolação linear no tempo (6.9) para as condições de contorno de $\widetilde{\mathbf{u}}$ na solução da equação (6.2). ( $R e=0.1$, $\delta t_{1}=2.0 \times 10^{-3} s, \delta t_{2}=1.0 \times 10^{-3} s$ e $\left.\delta t_{3}=5.0 \times 10^{-4} s\right)$.

\begin{tabular}{|c|c|c|c|c|c|c|}
\hline & $\delta t_{1}$ & $N_{1}$ & $\delta t_{2}$ & $N_{2}$ & $\delta t_{3}$ & $N_{m} \simeq$ \\
\hline velocidade- $u$ & & & & & & \\
$l_{2}$ & $4.488 \times 10^{-5}$ & 0.75 & $2.653 \times 10^{-5}$ & 0.88 & $1.433 \times 10^{-5}$ & 0.82 \\
$l_{1}$ & $3.817 \times 10^{-4}$ & 0.77 & $2.225 \times 10^{-4}$ & 0.89 & $1.195 \times 10^{-4}$ & 0.83 \\
$l_{\infty}$ & $1.136 \times 10^{-5}$ & 0.73 & $6.837 \times 10^{-6}$ & 0.87 & $3.721 \times 10^{-6}$ & 0.80 \\
velocidade- $v$ & & & & & & \\
$l_{2}$ & $3.372 \times 10^{-5}$ & 0.72 & $2.034 \times 10^{-5}$ & 0.87 & $1.110 \times 10^{-5}$ & 0.80 \\
$l_{1}$ & $2.572 \times 10^{-4}$ & 0.76 & $1.510 \times 10^{-4}$ & 0.89 & $8.150 \times 10^{-5}$ & 0.82 \\
$l_{\infty}$ & $8.568 \times 10^{-6}$ & 0.58 & $5.724 \times 10^{-6}$ & 0.81 & $3.250 \times 10^{-6}$ & 0.69 \\
pressão- $p$ & & & & & & \\
$l_{2}$ & $7.274 \times 10^{-4}$ & 0.91 & $3.928 \times 10^{-4}$ & 0.88 & $2.077 \times 10^{-4}$ & 0.90 \\
$l_{1}$ & $7.080 \times 10^{-3}$ & 0.80 & $4.055 \times 10^{-3}$ & 0.90 & $2.167 \times 10^{-5}$ & 0.85 \\
$l_{\infty}$ & $3.360 \times 10^{-4}$ & 1.32 & $1.340 \times 10^{-4}$ & 1.23 & $5.674 \times 10^{-5}$ & 1.28 \\
\hline
\end{tabular}

tese. Por aplicar apenas um tipo de equação para calcular $\psi$ na superfície livre, o sistema linear resultante agora é simétrico. Portanto esta formulação é mais robusta e apresenta resultados semelhantes às formulações anteriores. 
Tabela 6.7: Erros para a laplaciano superficial da seção 6.3 com a equação da pressão na superfície livre discretizada no nível de tempo $(n+1)$. $\left(R e=0.1, \delta t_{1}=2.0 \times 10^{-3} s, \delta t_{2}=\right.$ $1.0 \times 10^{-3} s$ e $\delta t_{3}=5.0 \times 10^{-4} s$.

\begin{tabular}{|c|c|c|c|c|c|c|}
\hline & $\delta t_{1}$ & $N_{1}$ & $\delta t_{2}$ & $N_{2}$ & $\delta t_{3}$ & $N_{m} \simeq$ \\
\hline $\begin{array}{c}\text { velocidade- } u \\
l_{2}\end{array}$ & $9.281 \times 10^{-6}$ & 0.97 & $4.724 \times 10^{-6}$ & 0.94 & $2.446 \times 10^{-6}$ & 0.96 \\
$l_{1}$ & $8.845 \times 10^{-5}$ & 1.05 & $4.250 \times 10^{-5}$ & 1.01 & $2.097 \times 10^{-5}$ & 1.03 \\
$l_{\infty}$ & $2.249 \times 10^{-6}$ & 1.10 & $1.045 \times 10^{-6}$ & 0.81 & $5.926 \times 10^{-7}$ & 0.96 \\
velocidade- $v$ & & & & & & \\
$l_{2}$ & $7.440 \times 10^{-6}$ & 1.03 & $3.631 \times 10^{-6}$ & 0.96 & $1.865 \times 10^{-6}$ & 0.99 \\
$l_{1}$ & $7.402 \times 10^{-5}$ & 1.07 & $3.503 \times 10^{-5}$ & 1.02 & $1.720 \times 10^{-5}$ & 1.05 \\
$l_{\infty}$ & $1.870 \times 10^{-6}$ & 1.13 & $8.530 \times 10^{-7}$ & 0.94 & $4.439 \times 10^{-7}$ & 1.03 \\
pressão- $p$ & & & & & & \\
$l_{2}$ & $4.952 \times 10^{-4}$ & 1.46 & $1.790 \times 10^{-4}$ & 1.30 & $7.248 \times 10^{-5}$ & 1.38 \\
$l_{1}$ & $3.339 \times 10^{-3}$ & 1.30 & $1.356 \times 10^{-3}$ & 1.19 & $5.935 \times 10^{-4}$ & 1.24 \\
$l_{\infty}$ & $2.333 \times 10^{-4}$ & 1.50 & $8.234 \times 10^{-5}$ & 1.38 & $3.149 \times 10^{-5}$ & 1.44 \\
\hline
\end{tabular}

Tabela 6.8: Erros para a formulação laplaciano superficial da seção 6.3 sem a extrapolação linear no tempo (6.9) e com a equação da pressão na superfície livre discretizada no nível de tempo $(n+1) .\left(R e=0.1, \delta t_{1}=2.0 \times 10^{-3} s, \delta t_{2}=1.0 \times 10^{-3} s\right.$ e $\left.\delta t_{3}=5.0 \times 10^{-4} s\right)$.

\begin{tabular}{|c|c|c|c|c|c|c|}
\hline & $\delta t_{1}$ & $N_{1}$ & $\delta t_{2}$ & $N_{2}$ & $\delta t_{3}$ & $N_{m} \simeq$ \\
\hline velocidade- $u$ & & & & & & \\
$l_{2}$ & $5.280 \times 10^{-5}$ & 0.77 & $3.086 \times 10^{-5}$ & 0.89 & $1.661 \times 10^{-5}$ & 0.83 \\
$l_{1}$ & $4.376 \times 10^{-4}$ & 0.79 & $2.530 \times 10^{-4}$ & 0.89 & $1.355 \times 10^{-4}$ & 0.84 \\
$l_{\infty}$ & $1.287 \times 10^{-5}$ & 0.74 & $7.675 \times 10^{-6}$ & 0.86 & $4.212 \times 10^{-6}$ & 0.80 \\
velocidade- $v$ & & & & & & \\
$l_{2}$ & $3.938 \times 10^{-5}$ & 0.74 & $2.348 \times 10^{-5}$ & 0.87 & $1.277 \times 10^{-5}$ & 0.81 \\
$l_{1}$ & $2.982 \times 10^{-4}$ & 0.77 & $1.740 \times 10^{-4}$ & 0.89 & $9.379 \times 10^{-5}$ & 0.83 \\
$l_{\infty}$ & $1.004 \times 10^{-5}$ & 0.62 & $6.511 \times 10^{-6}$ & 0.82 & $3.664 \times 10^{-6}$ & 0.72 \\
pressão- $p$ & & & & & & \\
$l_{2}$ & $7.447 \times 10^{-4}$ & 0.97 & $3.787 \times 10^{-4}$ & 0.95 & $1.949 \times 10^{-4}$ & 0.96 \\
$l_{1}$ & $7.121 \times 10^{-3}$ & 0.88 & $3.865 \times 10^{-3}$ & 0.91 & $2.051 \times 10^{-3}$ & 0.89 \\
$l_{\infty}$ & $3.418 \times 10^{-4}$ & 1.32 & $1.361 \times 10^{-4}$ & 1.23 & $5.768 \times 10^{-5}$ & 1.28 \\
\hline
\end{tabular}




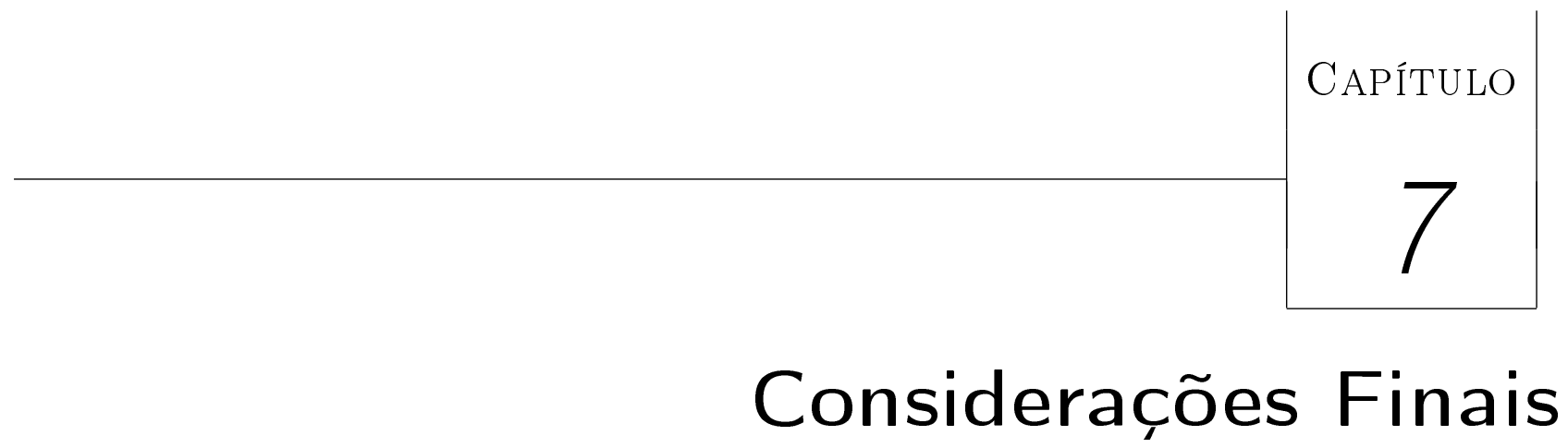

Esta tese foi dividida em três partes: análise de estabilidade de métodos numéricos em malhas deslocadas, construção de técnicas implícitas e estudos de métodos de projeção para escoamentos com superfícies livres. O desenvolvimento destas etapas foi baseada na metodologia MAC, e as implementações computacionais foram introduzidas no sistema FREEFLOW.

A primeira etapa foi apresentada no capítulo 3, no qual um estudo minucioso foi realizado, baseado em resultados de álgebra linear aplicada e em um problema modelo. Neste estudo, mostrou-se que quando o método de Crank-Nicolson é aplicado em uma malha deslocada este pode tornar-se instável. Os resultados para o problema modelo predizem em termos quantitativos o comportamento do método de Crank-Nicolson quando aplicado às equações de Navier-Stokes. O principal resultado obtido foi a demonstração de que é necessário discretizar implicitamente também as condições de contornos rígidos para que o método torne-se incondicionalmente estável.

As outras duas etapas foram apresentadas nos capítulos 4, 5 e 6 . Inicialmente, no capítulo 4, foram construidas técnicas implícitas para tratar a condição de tensão normal na superfície livre. Como consequência do tratamento implícito da equação da pressão na superfície livre ocorreu um acoplamento entre os campos de velocidade e pressão. Logo, métodos de projeção para escoamentos com superfícies livres foram construídos. Os resultados numéricos do capítulo 4 mostraram que os métodos desenvolvidos são estáveis e robustos, permitindo economia de tempo de simulação em escoamentos com superfícies livres e baixo número de Reynolds. 
No capítulo 5, as técnicas do capítulo 4 foram estendidas para o caso tridimensional. A estratégia de discretização implícita da condição de tensão normal à superfície livre foi novamente utilizada. Neste caso, novos métodos de projeção para escoamentos tridimensionais newtonianos e viscoelásticos foram derivados. Os métodos foram verificados em simulações tridimensionais com geometria complexa e $R e<<1$. Os resultados numéricos evidenciam a potencialidade dos métodos desenvolvidos para escoamentos de fluidos newtonianos e para fluidos do tipo Maxwell e Oldroyd-B.

O capítulo 6 foi dedicado à obtenção de métodos de projeção de segunda ordem para escoamentos com superfície livre. Foram investigadas várias estratégias resultando em uma nova metodologia denominada de laplaciano superficial, que apresentou vantagens em relação às formulações anteriores para simulações de escoamentos com superfície livre e baixo número de Reynolds.

Portanto, com a combinação de todas as estratégias desenvolvidas neste projeto de doutorado, é possível derivar novas técnicas em DFC incompressível para simulação numérica de escoamentos com superfícies livres.

Os principais trabalhos futuros relacionados a cada etapa desta tese são:

- Etapa 1: estudar a estabilidade do método Crank-Nicolson com diferentes tipos de condições de contorno em problemas com coeficiente de difusão transiente.

- Etapa 2: explorar novas estratégias para desacoplar o sistema linear não-simétrico para $\psi$, resultante da aplicação das técnicas implícitas na condição de tensão normal à superfície livre, e testar as formulações semi-implícitas em outros modelos viscoelásticos.

- Etapa 3: verificar se a ordem de precisão temporal é afetada em problemas com movimentação de superfície livre, desenvolver uma estratégia para desacoplar a velocidade normal $\left(u_{n}\right)$ e $\psi$ na equação da pressão na superfície livre na formulação laplaciano superficial, e aprofundar os estudos sobre o laplaciano superficial bidimensional, verificando se é possível estendê-lo para o caso tridimensional.

- Além destes estudos, investigar o refinamento de malha adaptativo e a implementação paralela nas formulações desenvolvidas para escoamentos com superfície livre. 


\section{Referências Bibliográficas}

[1] Y.C. Ahn e M.E. Ryan. A finite difference analysis of the extrudate swell problem. International Journal for Numerical Methods in Fluids, 13:1289-1310, 1991.

[2] A.S. Almgren, J.B. Bell, e W.Y. Crutchfield. Approximate projection methods: Part i. inviscid analysis. SIAM Journal on Scientific Computing, 22:1139-1159, 2000.

[3] M. Alves, P. Oliveira, e F.T. Pinho. A convergent and universally bounded interpolation scheme for the treatment of advection. International Journal for Numerical Methods in Fluids, 41:47-75, 2003.

[4] A.A. Amsden e F.H. Harlow. A simplified MAC technique for incompressible fluid flow calculations. Journal of Computational Physics, 6:332-335, 1970.

[5] V. Armenio. An improved MAC method (SIMAC) for unsteady high-Reynolds free surface flows. International Journal for Numerical Methods in Fluids, 24:185-214, 1997.

[6] S. Armfield e R. Street. An analysis and comparison of the time accuracy of fractionalstep methods for the Navier-Stokes equations on staggered grids. International Journal for Numerical Methods in Fluids, 38:255-282, 2002.

[7] S. Armfield e R. Street. The pressure accuracy of fractional-step methods for the NavierStokes equations on staggered grids. ANZIAM. Australian and New Zealand Industrial and Applied Mathematics, 44:20-39, 2003.

[8] P. Badiei, M.M. Namin, e A. Ahmadi. A three-dimensional non-hydrostatic vertical boundary fitted model for free-surface flows. International Journal for Numerical Methods in Fluids, 56:607-627, 2008.

[9] E. Bansch. Simulation of instationary, incompressible flows. Acta Math. Univ. Comenianae, LXVII:101-114, 1998. 
[10] G.K. Batchelor. An Introduction to Fluid Dynamics. SIAM, Philadelphia, 1970.

[11] J.B. Bell, P. Colella, e H.M. Glaz. A second order projection method for the incompressible Navier-Stokes equations. Journal of Computational Physics, 85:257-283, 1989.

[12] H. Bijl, M.H. Ampenter, V.N. Vatsa, e C.A. Kennedy. Implicit time integration schemes for the unsteady compressible Navier-Stokes equations: laminar flows. Journal of Computational Physics, 179:313-329, 2002.

[13] D.L. Brown, R. Cortez, e M.L. Minion. Accurate projection methods for the incompressible Navier-Stokes equations. Journal of Computational Physics, 168:464-499, 2001.

[14] L.S. Caretto, A.D. Gosman, S.V. Patankar, e D.B. Spalding. Two calculation procedures for steady, three dimensional flows with recirculation. Lectures Notes in Physics, 19, 1972.

[15] A.F. Castelo, M.F. Tomé, M.L. Cesar, J.A. Cuminato, e S. McKee. Freeflow: An intregated simulation system for three-dimensional free surface flows. Computing and Visualization in Science, 2:199-210, 2000.

[16] H.D. Ceniceros e A.M. Roma. A multi-phase flow method with a fast, geometry-based fluid indicator. Journal of Computational Physics, 205:391-400, 2005.

[17] R.K.C. Chan e R.L. Street. A computer study of finite-amplitude water waves. Journal of Computational Physics, 6:68-94, 1970.

[18] P.W. Chang, T.W. Patten, e B.A. Finlayson. Collocation and galerkin finite element methods for viscoelastic fluid flow - ii: Die swell problems with a free surface. Computers E) Fluids, 7:285-293, 1979.

[19] W. Chang, F. Giraldo, e B. Perot. Analysis of an exact fractional step method. Journal of Computational Physics, 180:183-199, 2002.

[20] T. Chen, P.D. Minev, e K. Nandakumar. A projection scheme for incompressible multiphase flow using adaptative Eulerian grid. International Journal for Numerical Methods in Fluids, 45:1-19, 2004.

[21] I. L. Chern, J. Glimm, O. Mcbryan, B. Plohr, e S. Yaniv. Front tracking for gas dynamics. Journal of Computational Physics, 62:83-110, 1986.

[22] A.J. Chorin. A numerical method for solving incompressible viscous flow problems. Journal of Computational Physics, 2:12-26, 1967. 
[23] A.J. Chorin. Numerical solution of the Navier-Stokes. Mathematics of Computation, 2:745-762, 1968.

[24] R. Codina. Pressure stability in fractional step finite element methods for incompressible flows. Journal of Computational Physics, 170:112-140, 2001.

[25] A.H. Coppola-Owen e R. Codina. A finite element model for free surface flows on fixed meshes. International Journal for Numerical Methods in Fluids, 54:1151-1171, 2007.

[26] R. Cortez. An impulse-based approximation of fluid motion due to boundary forces. Journal of Computational Physics, 123:341, 1996.

[27] R. Courant, K. Friedrichs, e H. Lewy. On the partial differential equations of mathematical physics. IBM Journal Research and Development, 11:215-234, 1967.

[28] M.J. Crochet e R. Keunings. Die swell of a Maxwell fluid: Numerical prediction. Journal of Non-Newtonian Fluid Mechanics, 7:199-212, 1980.

[29] M.J. Crochet e R. Keunings. Finite element analysis of die swell of a highly elastic fluid. Journal of Non-Newtonian Fluid Mechanics, 10:339-356, 1982.

[30] M.J. Crochet e R. Keunings. On numerical die swell calculation. Journal of NonNewtonian Fluid Mechanics, 10:85-94, 1982.

[31] J.O. Cruickshank e B.R. Munson. Viscous fluid buckling of plane and axisymmetric jets. Journal of Fluid Mechanics, 113:221-239, 1981.

[32] J.M. Danskin. The theory of max-min with applications. SIAM Journal on Applied Mathematics, 14:641-664, 1966.

[33] P. Dartzi, Y.S. Yang, e C.H. Chang. Computation of internal flow with free surface using artificial compressibility. Numerical Heat Transfer B, 33:119-134, 1998.

[34] D. Degani e C. Gutfinger. Three-dimensional low Reynolds number flows with a free surface. Journal of Computational Physics, 24:108-114, 1977.

[35] F.M. Denaro. On the applications of the Helmoltz-Hodge decomposition in projection methods for incompressible flows with general boundary conditions. International Journal for Numerical Method in Fluid, 43:43-69, 2003. 
[36] M. Deville, L. Kleiser, e F.M. Rannou. Pressure and time treatment for Chebyshev spectral solution of a Stokes problem. International Journal for Numerical Methods in Fluids, 4:1149-1163, 1984.

[37] M.O. Deville. An alternating direction implicit algorithm for viscous free surface flows. J. de Mécanique, 14:161-187, 1975.

[38] J.K. Dukowicz e A. S. Dvinsky. Approximate factorization as a high order splitting for the implicit incompressible flow equations. Journal of Computational Physics, 102:336-347, 1992.

[39] A. Dutta e M.E. Ryan. Dynamics of a creeping newtonian jet with gravity and surface tension: a finite difference technique for solving steady free-surface flows using orthogonal curvilinear coordinates. American Institute of Chemical Engineers Journal, 28:220-232, 1982.

[40] W. E e J.G. Liu. Finite difference schemes for incompressible flows in the velocity-impulse density formulation. Journal of Computational Physics, 130:67-76, 1997.

[41] A. Esmaeeli e G. Tryggvason. Direct numerical simulations of bubbly flows. Part 1: Low Reynolds number arrays. Journal of Fluid Mechanics, 377:313-345, 1998.

[42] M.W. Evans e F.H. Harlow. The particles-in-cell method for hydrodynamic calculations. Los Alamos National Laboratory Report, LA-2139, 1957.

[43] R. Fedkiw. Coupling an Eulerian fluid calculation to a Lagrangian solid calculation with the ghost fluid method. Journal of Computational Physics, 175:200-224, 2002.

[44] V.G. Ferreira, A.C. Brandi, F.A. Kurokawa, P. Seleghim Junior, A. Castelo, e J.A. Cuminato. Incompressible turbulent flow simulation using $\kappa-\epsilon$ model and upwind schemes. Mathematical Problems in Engineering, 2007:ID 12741, 2007.

[45] V.G. Ferreira, F.A. Kurokawa, C.M. Oishi, M.K. Kaibara, A. Castelo, e J.A. Cuminato. Evaluation of a bounded high order upwind scheme for $3 \mathrm{~d}$ incompressible free surface flow computations. Mathematics and Computers in Simulation, aceito para publicação, 2008, DOI: 10.1016/j.matcom.2007.04.018.

[46] V.G. Ferreira, F.A. Kurokawa, R.A.B. Queiroz, M.K. Kaibara, C.M. Oishi, J.A. Cuminato, A. Castelo, M.F. Tomé, e S. McKee. Assessment of a high-order finite difference 
upwind scheme for the simulation of convection-diffusion problems. International Journal for Numerical Methods in Fluids, aceito para publicação, 2008, DOI: 10.1002/fld.1875.

[47] V.G. Ferreira, N. Mangiavacchi, M.F. Tomé, A. Castelo, J.A. Cuminato, e S. McKee. Numerical simulation of turbulent free surface flow with two-equation $\kappa-\varepsilon$ eddy-viscosity models. International Journal for Numerical Methods in Fluids, 44:347-375, 2004.

[48] V.G. Ferreira, C.M. Oishi, F.A. Kurokawa, M.K. Kaibara, J.A. Cuminato, A. Castelo, M.F. Tomé, N. Mangiavacchi, e S. McKee. A combination of implicit and adaptative upwind tools for the numerical solution of incompressible free surface flows. Communications in Numerical Methods in Engineering, 23:419-445, 2007.

[49] V.G. Ferreira, M.F. Tomé, N. Mangiavacchi, A. Castelo, J.A. Cuminato, A.O. Fortuna, e S. McKee. High order upwinding and the hydraulic jump. International Journal for Numerical Methods in Fluids, 39:549-583, 2002.

[50] J.H. Ferziger e M. Períc. Computational Methods for Fluid Dynamics. Springer-Verlag, 1999.

[51] M. Fortin, R. Peyret, e R. Temam. Calcul des ecoulementes d'un fluide visquex incompressible. J. Mec., 10:357-390, 1971.

[52] A.O. Fortuna. Técnicas Computacionais para Dinâmica dos Fluidos. Edusp, 2000.

[53] G.C. Georgiou e A.G. Boudouvis. Converged solutions of the Newtonian extrudate-swell problem. International Journal for Numerical Methods in Fluids, 29:363-371, 1999.

[54] W.A. Gifford. The effect of surface tension on extrudate swell from square and retangular channels. Polymer Engineering and Science, 38:1167-1174, 1998.

[55] J. Glimm, J. Grove, B. Lindquist, O. McBryan, e G. Tryggvason. The bifurcation of tracked scalar waves. SIAM Journal on Scientific and Statistical Computing, 9:61-79, 1988.

[56] J. Glimm, J. W. Grove, X. L. Li, K. M. Shyue, Y. Zeng, e Q. Zhang. Three-dimensional front tracking. SIAM Journal on Scientific Computing, 19:703-727, 1998.

[57] J. Glimm, J. W. Grove, e Y. Zhang. Interface tracking for axisymmetric flows. SIAM Journal on Scientific Computing, 24:208-236, 2002. 
[58] M. Golafashani. A simple numerical technique for transient creep flows with free surfaces. International Journal for Numerical Methods in Fluids, 8:897-912, 1988.

[59] P.M. Gresho. On the theory of semi-implicit projection methods for viscous incompressible flow and its implementation via a finite element method that also introduces a nearly consistent mass matrix. Part 1: Theory. International Journal for Numerical Methods in Fluids, 11:587-620, 1990.

[60] M. Griebel, T. Dornseifer, e T. Neunhoeffer. Numerical Simulation in Fluid Dynamics: A Practical Introduction. Society for Industrial and Applied Mathematics - SIAM, 1998.

[61] J.L. Guermond, P. Minev, e J. Shen. An overview of projection methods for incompressible flows. Computer Methods in Applied Mechanics and Engineering, 195:6011-6045, 2006.

[62] J.L. Guermond e J. Shen. A new class of truly consistent splitting schemes for incompressible flows. Journal of Computational Physics, 192:262-276, 2003.

[63] J.L. Guermond e J. Shen. On error estimates of rotational pressure-correction projection methods. Mathematics of Computations, 73:1719-1737, 2004.

[64] O. Guler. Foundations of Optimization in finite dimensions. Springer-Verlag, 2008.

[65] R.D. Guy e A.L. Fogelson. Stability of approximate projection methods on cell-centered grids. Journal of Computational Physics, 203:517-538, 2005.

[66] F.H. Harlow e J.E. Welch. Numerical calculation of time-dependent viscous incompressible flow of fluid with free surface. Physics of Fluids, 8:2182-2189, 1965.

[67] M.O. Henriksen e J. Holmen. Algebraic splitting for incompressible Navier-Stokes equations. Journal of Computational Physics, 175:438-453, 2002.

[68] G.A. Hill e C.L. Chenier. Die swell experiments for Newtonian fluids. The Canadian Journal of Chemical Engineering, 62:40-45, 1984.

[69] C. Hirsch. Numerical Computational of Internal and External Flows, vol. 1. A WileyInterscience Publication, 1989.

[70] C.W. Hirt, A.A Amsden, e J.L. Cook. An arbitrary Lagrangian-Eulerian computing method for all flow speeds. Journal of Computational Physics, 14:227-253, 1974.

[71] C.W. Hirt e B.D. Nichols. Volume-Of-Fluid (VOF) method for the dynamics of free boundaries. Journal of Computational Physics, 39:201-225, 1981. 
[72] W.V.D. Hodge. The Theory and Applications of Harmonic Integrals. Cambridge University Press, Cambridge, 1952.

[73] R.A. Horn e C.R. Johnson. Matrix Analysis. Cambridge University Press, Cambridge, 1996.

[74] K. Housiadas, G. Georgiou, e J. Tsamopoulos. The steady annular extrusion of a Newtonian liquid under gravity and surface tension. International Journal for Numerical Methods in Fluids, 33:1099-1119, 2000.

[75] S.X. Huang e C.J. Lu. Stress relaxation characteristics and extrudate swell of the iupacldpe melt. Journal of Non-Newtonian Fluid Mechanics, 136:147-156, 2006.

[76] P. Iannelli e F.M. Denaro. Analysis of the local truncation error in the pressure-free projection method for incompressible flows: a new accurate expression of the intermediate boundary conditions. International Journal for Numerical Methods in Fluids, 42:399-437, 2003.

[77] P.T. Jimack. Adaptive algorithms for free surface flow. Proceedings of the Fourth International Conference on Engineering Computational Technology, 2004.

[78] J. Van Kan. A second-order accurate pressure-correction schemes for viscous incompressible flow. SIAM Journal on Scientific and Statistical Computing, 7:870-891, 1986.

[79] G. Karniadakis, M. Israeli, e S. A. Orszag. High-order splitting methods for the incompressible Navier-Stokes equations. Journal of Computational Physics, 97:414-443, 1991.

[80] J. Kim e P. Moin. Application of a fractional-step method to incompressible Navier-Stokes equations. Journal of Computational Physics, 59:308-323, 1985.

[81] S.O. Kim e H.C. No. Second-order model for free surface convection and interface reconstruction. International Journal for Numerical Methods in Fluids, 26:79-100, 1998.

[82] K.M. Kleefsman, G. Fekken, A.E.P. Veldman, B. Iwanowski, e B. Buchner. A VolumeOf-Fluid based simulation method for wave impact problems. Journal of Computational Physics, 206:363-393, 2005.

[83] W. Kress e P. Lötstedt. Time step restrictions using semi-explicit methods for incompressible Navier-Stokes equations. Computer Methods in Applied Mechanics and Engineering, 195:4433-4447, 2006. 
[84] Y.K. Kwok e K.K. Tam. Linearized stability analysis of staggered-grid difference schemes for multidimensional viscous incompressible flows. Numerical Methods for Partial Differential Equations, 9:313-322, 1993.

[85] O.A. Ladyzhenskaja. The mathematical theory of viscous incompressible flow. Gordon and Breach, New York, 1963.

[86] H.P. Langtangen, K.A. Mardal, e R. Winther. Numerical methods for incompressible viscous flow. Advances in Water Resources, 25:8-12, 2002.

[87] M. Lee, D. Oh, e Y.B Kim. Canonical fractional-step methods and consistent boundary conditions for the incompressible Navier-Stokes equations. Journal of Computational Physics, 168:73-100, 2001.

[88] C. Lemos. High-order schemes for free surface flows with arbitrary configurations. International Journal for Numerical Methods in Fluids, 23:545-566, 1996.

[89] R.W. Lewis, S.E. Navti, e C. Taylor. A mixed Lagrangian-Eulerian approach to modelling fluid flow during mould filling. International Journal for Numerical Methods in Fluids, 25:931-952, 1997.

[90] Z. Li e M.C. Lai. The immersed interface method for the Navier-Stokes equation with singular forces. Journal of Computational Physics, 171:822-842, 2001.

[91] J-Z. Liang. Estimation of melt shear modulus from extrudate swell ratio and exit-pressure drop data. Journal of Thermoplastic Composite Materials, 17:545-556, 2004.

[92] Y. Liang, A. Ozetkin, e S. Neti. Dynamics of viscoelastic jets of polymeric liquid extrudate. Journal of Non-Newtonian Fluid Mechanics, 81:105-132, 1999.

[93] P. Lin e D. Wang. Numerical modelling of 3d stratified free surface flows: a case study of sediment dumping. International Journal for Numerical Methods in Fluids, 50:1425-1444, 2006 .

[94] M. Liu, Y.X. Ren, e H. Zhang. A class of fully second order accurate projection methods for solving the incompressible Navier-Stokes equations. Journal of Computational Physics, 200:325-346, 2004.

[95] T.G. Liu, B.C. Khoo, e C.W. Wang. The ghost fluid method for compressible gas-water simulation. Journal of Computational Physics, 204:193-221, 2005. 
[96] T.J. Liu, A. Yu, e S.H. Cheng. Finite difference solution of a Newtonian jet swell problem. International Journal for Numerical Methods in Fluids, 12:125-142, 1991.

[97] D. Lorstad e L. Fuchs. High-order surface tension VOF-model for 3d bubble flows with high density ratio. Journal of Computational Physics, 200:153-176, 2004.

[98] N. Mangiavacchi, A. Castelo, M.F. Tomé, J.A. Cuminato, M.L.B. de Oliveira, e S. McKee. An effective implementation of surface tension using the Marker-And-Cell method for axisymmetric and planar flows. SIAM Journal on Scientific Computing, 26:1340-1368, 2005 .

[99] M. Manna e A. Vacca. An efficient method for the solution of the incompressible NavierStokes equations in cylindrical geometries. Journal of Computational Physics, 151:563584, 1999.

[100] S. McKee, M.F. Tomé, V.G. Ferreira, J.A. Cuminato, A. Castelo, F.S. Sousa, e N. Mangiavacchi. The MAC method. Computers \& Fluids, 37:907-930, 2008.

[101] E. Mitsoulis. Three-dimensional non-Newtonian computations of extrudate swell with the finite element method. Computer Methods in Applied Mechanics and Engineering, 180:333-344, 1999.

[102] H. Miyata. Finite-difference simulation of breaking waves. Journal of Computational Physics, 65:179-214, 1986.

[103] H. Miyata, S. Nishimura, e A. Masuko. Finite-difference simulation of nonlinear waves generated by ships of arbitrary three-dimensional configuration. Journal of Computational Physics, 60:391-436, 1985.

[104] J.P. Morris, P.J. Fox, e Y. Zhu. Modeling low Reynolds number incompressible flows using SPH. Journal of Computational Physics, 136:214-226, 1997.

[105] D.E. Morton, M.J. Rudman, e J.L. Liow. A finite difference method for modelling impacting drops. In Proceedings of FEDSM97, pages 1-6, 1997.

[106] M. Mäntylä. An introduction to solid modeling. Computer Science Press, Rockville, MD, 1988.

[107] V. Ngamaramvaranggul e M.F. Webster. Viscoelastic simulation of stick-slip and die-swell flows. International Journal for Numerical Methods in Fluids, 29:363-371, 1999. 
[108] M.J. Ni, S. Komori, e N. Morley. Projection methods for the calculation of incompressible unsteady flows. Numerical Heat Transfer, Part B, 44:533-551, 2003.

[109] B.D. Nichols e C.W. Hirt. Methods for calculating multidimensional, transient free surface flows past bodies. In Proceedings of the First International Conf. On Num. Ship Hydrodynamics, 1975.

[110] B.D. Nichols, C.W. Hirt, e R.S. Hotchkiss. Sola-VOF: a solution algorithm for transient fluid flow with multiple free boundaries. Los Alamos National Laboratory Report, LA8355, 1980.

[111] N. Nikitin. Third-order-accurate semi-implicit Runge-Kutta scheme for incompressible Navier-Stokes equations. International Journal for Numerical Methods in Fluids, 51:221$233,2006$.

[112] M. Nóbrega, O.S. Carneiro, F.T. Pinho, G.S. Paulo, M.F. Tomé, A. Castelo, e J.A. Cuminato. The phenomenon of jet buckling: experimental results and numerical predictions. Proceedings of The Polymer Processing Society 23rd Annual Meeting, Salvador/Brasil, 2007.

[113] C.M. Oishi. Análise e implementação de métodos implícitos no sistema FreeFlow2D. Dissertação de mestrado, Universidade de São Paulo, Brasil, 2004.

[114] C.M. Oishi, J.A. Cuminato, V.G. Ferreira, M.F. Tomé, A. Castelo, e N. Mangiavacchi. Implementing implicit schemes in GENSMAC. Tendências em Matemática Aplicada e Computacional, 5:257-266, 2004.

[115] C.M. Oishi, J.A. Cuminato, V.G. Ferreira, M.F. Tomé, A. Castelo, e N. Mangiavacchi. A study of numerical schemes for incompressible fluid flows. Tendências em Matemática Aplicada e Computacional, 6:111-120, 2005.

[116] C.M. Oishi, J.A. Cuminato, V.G. Ferreira, M.F. Tomé, A. Castelo, N. Mangiavacchi, e S. McKee. A stable semi-implicit method for free surface flows. Transaction of the ASME - Journal of Applied Mechanics, 73:940-947, 2006.

[117] C.M. Oishi, J.A. Cuminato, J.Y. Yuan, e S. McKee. Stability of numerical schemes on staggered grids. Numerical Linear Algebra with Applications, aceito para publicação, 2008, DOI: 10.1002/nla.597. 
[118] C.M. Oishi, M. F. Tomé, J.A. Cuminato, e S. McKee. An implicit technique for solving 3d low Reynolds number moving free surface flows. Journal of Computational Physics, 227:7446-7468, 2008.

[119] B.J. Omodei. Computer solutions of a plane Newtonian jet with surface tension. Computers \& Fluids, 7:79-96, 1979.

[120] S.A. Orszag, M. Israeli, e M. Deville. Boundary conditions for incompressible flows. SIAM Journal on Scientific Computing, 1:75-111, 1986.

[121] S. Osher e R. Fedkiw. Level set methods: An overview and some recent results. Journal of Computational Physics, 169:463-502, 2001.

[122] S.V. Patankar. Numerical Heat Transfer and Fluid Flows. Hemisphere Publishing Co., 1980 .

[123] G.S. Paulo, M.F. Tomé, e S. McKee. A Marker-And-Cell approach to viscoelastic free surface flows using the PTT model. Journal of Non-Newtonian Fluid Mechanics, 147:149$174,2007$.

[124] B. Perot e R. Nallapati. A moving unstructured staggered mesh method for the simulation of incompressible free-surface flows. Journal of Computational Physics, 184:192-214, 2003.

[125] J.B. Perot. An analysis of the fractional step method. Journal of Computational Physics, 108:51-58, 1993.

[126] J.B. Perot. Comments on the fractional-step method. Journal of Computational Physics, 121:179, 1995.

[127] N.A. Petersson. Stability of pressure boundary conditions for Stokes and Navier-Stokes equations. Journal of Computational Physics, 172:40-70, 2001.

[128] J.E. Pilliod e E.G. Puckett. Second-order accurate Volume-Of-Fluid algorithms for tracking material interface. Journal of Computational Physics, 199:465-502, 2004.

[129] W.E. Pracht. A numerical method for calculating transient creep flows. Journal of Computational Physics, 7:46-60, 1971.

[130] W.H. Press, B.P. Flannery, S.A. Teukolsky, e W.T. Vetterling. Numerical Recipes in $C$ : The Art of Scientific Computing, 2nd ed. Cambridge - University Press, 1992. 
[131] S. Prudhomme e J.T. Oden. Numerical stability and error analysis for the incompressible Navier-Stokes equations. Communications in Numerical Methods in Engineering, 18:779$787,2002$.

[132] J.H. Pyo e J. Shen. Normal mode analysis of second-order projection methods for incompressible flows. Discrete and Continuous Dynamical System - Series B, 5:817-840, 2005 .

[133] A. Quarteroni, R. Sacco, e F. Saleri. Numerical Mathematics. Springer, 2000.

[134] A. Quarteroni, F. Saleri, e A. Veneziani. Factorization methods for the numerical approximation of Navier-Stokes equations. Computer Methods in Applied Mechanics and Engineering, 188:505-526, 2000.

[135] P.E. Raad e R. Bidoae. The three-dimensional Eulerian-Lagrangian marker and micro cell method for the simulation of free surface flows. Journal of Computational Physics, 203:668-699, 2005.

[136] N.M. Ribe. Periodic folding of viscous jets. Physical Review E, 68:Art. No. 036305, 2003.

[137] N.M. Ribe. Coiling of viscous jets. Proceedings of the Royal Society of London Series A - Mathematical Physical and Engineering Sciences, 460:3223-3239, 2004.

[138] N.M. Ribe, H.E. Huppert, M.A. Hallworth, M. Habibie, e D. Bonn. Multiple coexisting states of liquid rope coiling. Journal of Fluid Mechanics, 555:275-297, 2006.

[139] R.P.G. Rutgers e M.R. Mackley. The effect of channel geometry and wall boundary conditions on the formation of extrusion surface instabilities for lldpe. Journal of NonNewtonian Fluid Mechanics, 98:185-199, 2001.

[140] J. Shen. On error estimates of the projection methods for the Navier-Stokes equations: first-order schemes. SIAM Journal on Numerical Analysis, 29:57-77, 1992.

[141] J. Shen. On error estimates of the projection methods for the Navier-Stokes equations: second-order schemes. Mathematics of Computations, 65:1039-1065, 1996.

[142] J.R. Shewchuk. An introduction to the conjugate gradient method without the agonizing pain. Technical report, CS-94-125, 1994. 
[143] R.A.P. Silva e M.F. Tomé. A numerical technique for simulating 3d free surface flows of an upper-convected Maxwell fluid. Proceedings of the XXVII Iberian Latin American Congress on Computational Methods in Engineering, Porto/Portugal, 2007.

[144] N. Sombatsompop e N.T. Intawong. A comparative study on extrudate swell ratio of polystyrene in a capillary rheometer and a single screw extruder. Polymer Testing, 24:948952, 2005.

[145] F.S. Sousa, N. Mangiavacchi, L.G. Nonato, A. Castelo, M.F. Tomé, V.G. Ferreira, J.A. Cuminato, e S. McKee. A front-tracking/front-capturing method for the simulation of 3d multi-fluid flows with free surfaces. Journal of Computational Physics, 198:469-499, 2004 .

[146] J.C. Strilkwerda e Y.S. Lee. The accuracy of the fractional step method. SIAM Journal on Numerical Analysis, 37:37-47, 1999.

[147] C. Tadjeran. Stability analysis of the Crank-Nicholson method for variable coefficient diffusion equation. Communications in Numerical Methods in Engineering, 23:29-34, 2007.

[148] N. Takemitsu. Finite difference method to solve incompressible fluid flow. Journal of Computational Physics, 61:499-518, 1985.

[149] R.I. Tanner. A theory of die-swell. Journal of Polymer Science Part A, 8:2067-2078, 1970.

[150] R.I. Tanner. Engineering Rheology. Clarendon Press, Oxford, 1988.

[151] E.Y. Tau. A second-order projection method for the incompressible Navier-Stokes equations in arbitrary domains. Journal of Computational Physics, 115:147-152, 1994.

[152] T. Temam. Sur l'approximation de la solution des equations de Navier-Stokes par la methode de pas fractionnaires (ii). Archieves of Rational Mechanics and Analysis, 33:377$385,1969$.

[153] K. Tenenblat. Introdução a geometria diferencial. Editora da UnB, 1988.

[154] L.J.P. Timmermans, P.D. Minev, e F.N. Van De Vosse. An approximate projection scheme for incompressible flow using spectral elements. International Journal for Numerical Methods in Fluids, 22:673-688, 1996. 
[155] M.F. Tomé, A. Castelo, J.A. Cuminato, N. Mangiavacchi, e S. McKee. Gensmac3d: a numerical method for solving unsteady three-dimensional free surface flows. International Journal for Numerical Methods in Fluids, 37:747-796, 2001.

[156] M.F. Tomé, A. Castelo, e V.G. Ferreira. Numerical solution of the oldroyd-b model for three-dimensional viscoelastic free surface flows. Proceedings of the ECCOMAS CFD 2006-European Conference on Computational Fluid Dynamics, Egmond Aan Zee/Netherlands, 2006.

[157] M.F. Tomé, A. Castelo, V.G. Ferreira, e S. McKee. A finite difference technique for solving the Oldroyd-B model for 3d-unsteady free surface flows. Journal of Non-Newtonian Fluid Mechanics, aceito para publicação, 2008.

[158] M.F. Tomé, J.L. Doricio, J.A. Cuminato, A. Castelo, e S. McKee. Solving viscoelastic free surface flows of a second-order fluid using a Marker-And-Cell approach. International Journal for Numerical Methods in Fluids, 53:599-627, 2007.

[159] M.F. Tomé, B. Duffy, e S. McKee. A numerical technique for solving unsteady nonNewtonian free surface flows. Journal of Non-Newtonian Fluid Mechanics, 62:9-34, 1996.

[160] M.F. Tomé, L. Grossi, A. Castelo, J. A. Cuminato, S. McKee, e K. Walters. Die-swell, splashing drop and a numerical technique for solving the oldroyd-b model for axisymmetric free surface flows. Journal of Non-Newtonian Fluid Mechanics, 141:147-165, 2007.

[161] M.F. Tomé, L. Grossi, A. Castelo, J.A. Cuminato, V.G. Ferreira, F.S. Sousa, e S. McKee. A numerical method for solving three-dimensional generalized Newtonian free surface flows. Journal of Non-Newtonian Fluid Mechanics, 123:85-103, 2004.

[162] M.F. Tomé, N. Mangiavacchi, J.A. Cuminato, A. Castelo, e S. McKee. A finite difference technique for simulating unsteady viscoelastic free surface flows. Journal of NonNewtonian Fluid Mechanics, 106:61-106, 2002.

[163] M.F. Tomé e S. McKee. Gensmac: A computational Marker-And-Cell method for free surface flows in general domains. Journal of Computational Physics, 110:171-186, 1994.

[164] M.F. Tomé, S. McKee, L. Barratt, D.A. Jarvis, e A.J. Patrick. An experimental and numerical investigations of container filling with viscous liquids. International Journal for Numerical Methods in Fluids, 31:1333-1353, 1999. 
[165] M.F. Tomé, R.A. Silva, C.M. Oishi, e S. McKee. Numerical solution of the upperconvected Maxwell model for three-dimensional free surface flows. Communication on Computational Physics, submetido, 2008.

[166] F. Tone e D. Wirosoetisno. On the long-time stability of the implicit Euler scheme for the two-dimensional Navier-Stokes equations. SIAM Journal on Numerical Analysis, 44:29-40, 2006.

[167] G. Tryggvason, B. Burner, e A. Esmaeeli. A front tracking method for the computations of multiphase flow. Journal of Computational Physics, 169:708-759, 2001.

[168] G. Tryggvason, B. Burner, A. Esmaeeli, D. Juric, e N. Al-Rawahi. Computations of multiphase flow. Advances in Applied Mechanics, 39:81-120, 2003.

[169] S. Turek. A comparative study of some time-stepping techniques for the incompressible Navier-Stokes equations: From fully implicit nonlinear schemes to semi-implicit projection methods. International Journal for Numerical Methods in Fluids, 22:987-1011, 1996.

[170] S. Ushijima. Arbitrary Lagrangian Eulerian numerical prediction for local scour caused by turbulent flows. Journal of Computational Physics, 125:71-82, 1996.

[171] J. Viecelli. A method for including arbitrary external boundaries in the MAC incompressible fluid computing technique. Journal of Computational Physics, 4:543-551, 1969.

[172] S. Vincent e J.P. Caltagirone. Efficient solving method for unsteady incompressible interfacial flow problems. International Journal for Numerical Methods in Fluids, 30:795-811, 1999 .

[173] C. Vuik, A. Saghir, e G.P. Boerstoel. The Krylov accelerated SIMPLE(R) method for flow problems in industrial furnaces. International Journal for Numerical Methods in Fluids, 33:1027-1040, 2000.

[174] E. Weinan e J. Liu. Projection method i: Convergence and numerical boundary layers. SIAM Journal of Numerical and Analytical, 32:1017-1057, 1995.

[175] B.R. Wetton. Error analysis for Chorin's original method with regularization in space and time. SIAM Journal on Numerical Analysis, 34:1683-1697, 1997.

[176] N.N. Yanenko. The Method of Fractional Steps. Springer-Verlag, New York, 1971. 
[177] B. Yang e A. Prosperetti. A second-order boundary-fitted projection method for freesurface flow computations. Journal of Computational Physics, 213:574-590, 2006.

[178] Y. Yang e H.S. Udaykumar. Sharp interface cartesian grid method ii: solidification of pure materials and binary solutions. Journal of Computational Physics, 210:55-74, 2005.

[179] T. Ye, W. Shyy, e J. Chung. A fixed-grid, sharp-interface method for bubble dynamics and phase change. Journal of Computational Physics, 174:781-815, 2001.

[180] W.C. Yueh. Eigenvalues of several tridiagonal matrices. Applied Mathematics E-Notes, 5:66-74, 2005.

[181] K.K.Q. Zhang. A discrete splitting finite element method for numerical simulations of incompressible Navier-Stokes equations. International Journal for Numerical Methods in Fluids, 64:285-303, 2005. 


$\bar{A}$

\section{Aspectos importantes da discretização} espacial

\section{A.1 Método GENSMAC}

Detalhes sobre as equações de diferenças finitas do algoritmo GENSMAC, apresentado anteriormente na seção 2.7, podem ser encontrados em [163] para o caso bidimensional e em [155] para o caso tridimensional. Entretanto, nesta seção serão apresentados alguns aspectos importantes na discretização espacial para uma malha deslocada.

A equação de quantidade de movimento (2.25) é discretizada nas arestas da célula no caso bidimensional, e discretizada nas faces no caso tridimensional. A equação da continuidade (2.2) é discretizada no centro da célula. Os termos viscosos e o gradiente de pressão da equação de quantidade de movimento (2.25) são aproximados por diferenças centrais de segunda ordem. A discretização espacial dos termos não-lineares em (2.25) requer atenção especial quando as equações de Navier-Stokes são resolvidas numericamente. Isto porque, muitas vezes, os termos não-lineares, conhecidos como termos advectivos ou convectivos, podem introduzir oscilações na solução numérica. Detalhes sobre esquemas numéricos para o tratamento dos termos convectivos na formulação GENSMAC foram apresentados em $[49,48]$ para o caso bidimensional e em $[45,46]$ para o caso tridimensional.

Na seção 2.5 foi apresentada a metodologia para aproximar a superfície livre para o caso bidimensional (figuras 2.4, 2.5 e 2.6) e tridimensional (figuras 2.7, 2.8 e 2.9). Entretanto, naquele 
momento, não foi apresentada a forma de utilizar as componentes das velocidades normal e tangencial em uma célula de superfície livre nas equações de diferenças finitas. Desta forma, um aspecto importante nos esquemas baseados na metodologia MAC é definir onde se situam as componentes das velocidades normal e tangencial em uma célula da malha deslocada na superfície livre. Para simplicar, considera-se o caso bidimensional situando estas componentes de acordo com a figura A.1 para o caso vertical, figura A.2 para o caso horizontal e a figura A.3 para o caso inclinado.
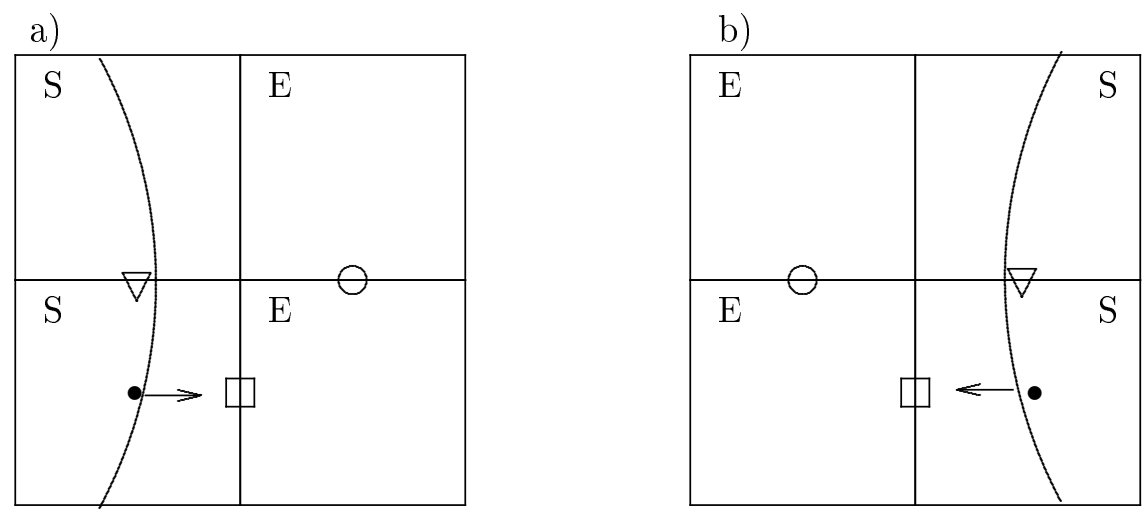

$$
\begin{aligned}
& \square \text { velocidade normal à superfície }\left(u_{n}\right) \\
& \bigcirc \text { velocidade tangencial à superfície }\left(v_{m}\right) \\
& \nabla \text { velocidade pertencente ao domínio }(v) \\
& \text { - ponto }(i, j) \text { : quantidade escalar } \\
& >\text { vetor normal }(\mathbf{n})
\end{aligned}
$$

Figura A.1: Localização das componentes de velocidade e da quantidade escalar em células próximas à superfície livre. Orientação vertical determinada pelos vetores: a) $\mathbf{n}=(1,0)$ ou b) $\mathbf{n}=(-1,0)$.

Conforme as figuras A.1, A.2 e A.3, é possível verificar que em cada célula [S], independente da relação com a célula vizinha $[\mathbf{E}]$, existe uma velocidade normal e uma tangencial à superfície livre, localizadas nas arestas das células, e um escalar, localizado no centro da célula [S] em questão. De acordo com Tomé e McKee [163], no caso bidimensional a velocidade normal como ilustrado nas figuras A.1, A.2 e A.3 é calculada pela equação da continuidade (2.2), enquanto a velocidade tangencial pela equação (2.12). Como exemplo da aplicação destas equações, considere a figura A.2a). Neste caso, aplicando a equação da continuidade no ponto $(i, j)$, a velocidade normal, denotada na figura A.2a) com $\square$, é calculada como

$$
v_{n}=v_{i, j-\frac{1}{2}}-\frac{\delta y}{\delta x}\left(u_{i+\frac{1}{2}, j}-u_{i-\frac{1}{2}, j}\right),
$$



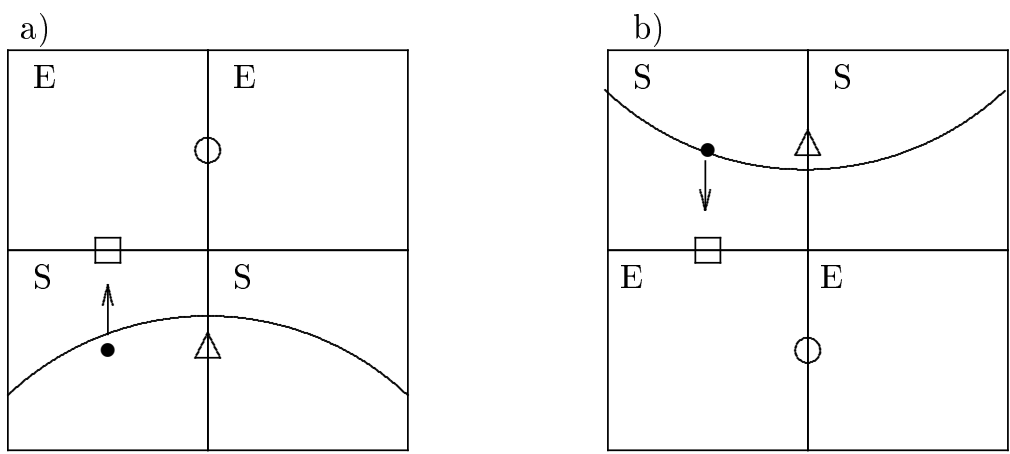

$\square$ velocidade normal à superfície $\left(v_{n}\right)$

$\bigcirc$ velocidade tangencial à superfície $\left(u_{m}\right)$

$\triangle$ velocidade pertencente ao domínio $(u)$

- $\operatorname{ponto}(i, j)$ : quantidade escalar

$\uparrow$ vetor normal (n)

Figura A.2: Localização das componentes de velocidade e da quantidade escalar em células próximas à superfície livre. Orientação horizontal determinada pelos vetores: a) $\mathbf{n}=(0,1)$ ou b) $\mathbf{n}=(0,-1)$.

onde $v_{n}=v_{i, j+\frac{1}{2}}$. A equação (2.12) nesta configuração reduz-se a

$$
\frac{\partial u}{\partial y}+\frac{\partial v}{\partial x}=0
$$

e quando aplicada no ponto $\left(i+\frac{1}{2}, j+\frac{1}{2}\right)$, a velocidade tangencial é calculada como

$$
u_{m}=u_{i+\frac{1}{2}, j}-\frac{\delta y}{\delta x}\left(v_{i+1, j+\frac{1}{2}}-v_{i, j+\frac{1}{2}}\right),
$$

onde $u_{m}=u_{i+\frac{1}{2}, j+1}$. Note que a velocidade normal calculada da equação (A.1) é utilizada em (A.3). A construção das equações de diferenças finitas para o cálculo das velocidades normal e tangencial nos demais casos é análoga. Detalhes desta construção estão em [163]. Esta idéia é estendida para o caso tridimensional e pode ser verificada em [155].

Uma dúvida que pode surgir em relação às velocidades tangenciais nas figuras A.1 e A.2 é: por quê estas velocidades tangenciais a superfície livre estão localizadas em faces que separam duas células $[\mathbf{E}]$ ? Para responder esta pergunta, inicialmente considere a equação de quantidade 

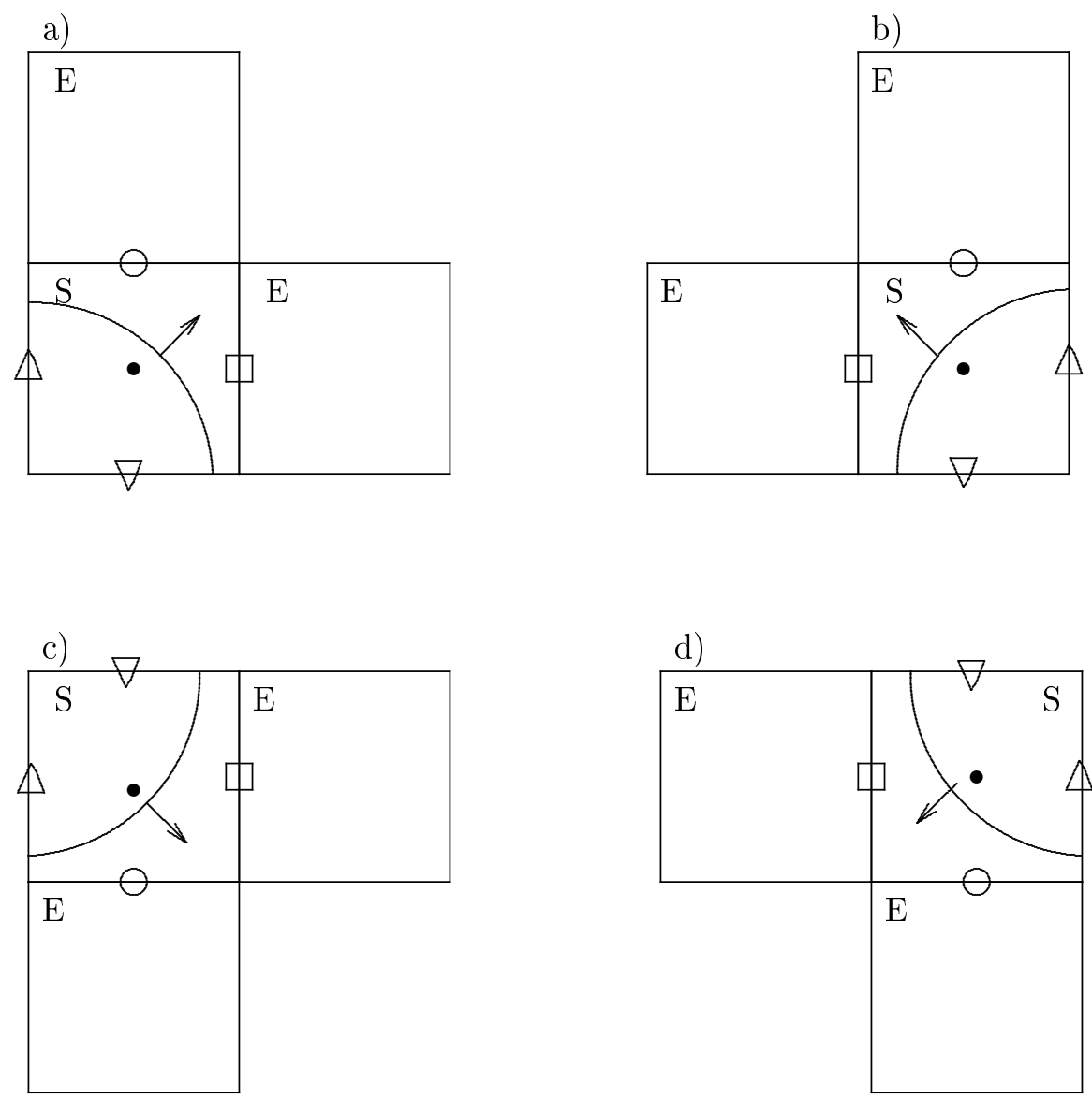

velocidade normal à superfície $\left(u_{n}\right)$

velocidade tangencial à superfície $\left(v_{m}\right)$

$\triangle \nabla$ velocidades pertencentes ao domínio $(u$ e $v)$

- $\operatorname{ponto}(i, j)$ : quantidade escalar

vetor normal $(\mathbf{n})$

Figura A.3: Localização das componentes de velocidade e da quantidade escalar em células próximas à superfície livre. Orientação inclinada determinada pelos vetores: a) $\mathbf{n}=\left(\frac{\sqrt{2}}{2}, \frac{\sqrt{2}}{2}\right)$, b) $\mathbf{n}=\left(-\frac{\sqrt{2}}{2}, \frac{\sqrt{2}}{2}\right)$, c) $\mathbf{n}=\left(\frac{\sqrt{2}}{2},-\frac{\sqrt{2}}{2}\right)$ e d) $\mathbf{n}=\left(-\frac{\sqrt{2}}{2},-\frac{\sqrt{2}}{2}\right)$. 
de movimento (2.25) na direção- $x$ discretizada no ponto $\left(i+\frac{1}{2}, j\right)$, ou seja,

$$
\begin{aligned}
\frac{1}{\delta t} \widetilde{u}_{i+\frac{1}{2}, j} & =\frac{1}{\delta t} u_{i+\frac{1}{2}, j}^{(n)}+\frac{1}{R e}\left(\frac{u_{i-\frac{1}{2}, j}-2 u_{i+\frac{1}{2}, j}+u_{i+\frac{3}{2}, j}}{(\delta x)^{2}}+\frac{u_{i+\frac{1}{2}, j-1}-2 u_{i+\frac{1}{2}, j}+u_{i+\frac{1}{2}, j+1}}{(\delta y)^{2}}\right)^{(n)} \\
& -(\mathcal{C}(u u)+\mathcal{C}(v u))^{(n)}-\left(\frac{p_{i+1, j}-p_{i, j}}{\delta x}\right)^{(n)}+\frac{1}{F r^{2}} g_{x}
\end{aligned}
$$

onde os termos convectivos $\mathcal{C}(u u)$ e $\mathcal{C}(v u)$ são aproximados por algum método upwind de alta ordem como descrito em Ferreira et al. [44].

Agora considere a equação de diferenças finitas (A.4) aplicada em uma célula da malha na superfície livre, como por exemplo na figura A.2. Neste caso, com os vetores $\mathbf{n}=(0,1)$ ou $\mathbf{n}=(0,-1)$, a velocidade $\widetilde{u}_{i+\frac{1}{2}, j}$ pertence ao domínio e está localizada entre duas células $[\mathbf{S}]$. Ou seja, a equação (A.4) deve ser discretizada no ponto $\left(i+\frac{1}{2}, j\right)$ da célula $[\mathbf{S}]$ pois a velocidade a ser determinada por esta equação não é considerada como uma velocidade de contorno, mas sim do domínio (ver $\triangle$ na figura A.2).

Lembrando que, o termo viscoso da equação (A.4) é aproximado pelo laplaciano de cinco pontos, a velocidade tangencial localizada no ponto $\left(i+\frac{1}{2}, j+1\right)$ para $\mathbf{n}=(0,1)$ (ou no ponto $\left(i+\frac{1}{2}, j-1\right)$ para $\left.\mathbf{n}=(0,-1)\right)$ será necessária, pois é uma das variáveis determinadas pela discretização espacial do laplaciano (veja a discretização do laplaciano na equação (A.4)).

Da mesma forma, para os vetores $\mathbf{n}=(1,0)$ ou $\mathbf{n}=(-1,0)$, na figura A.1, a equação de quantidade de movimento é aplicada na direção y na célula $[\mathbf{S}]$ em questão. Isto é, a equação (2.25) é discretizada no ponto $\left(i, j+\frac{1}{2}\right)$ da célula $[\mathbf{S}]$ pois a velocidade a ser calculada por esta equação é considerada como uma velocidade do domínio (ver $\nabla$ na figura A.1). Aproximando o termo viscoso da equação (2.25) discretizada na direção $y$, será necessário a velocidade tangencial localizada no ponto $\left(i+1, j+\frac{1}{2}\right)$ para $\mathbf{n}=(1,0)$ (ou no ponto $\left(i-1, j+\frac{1}{2}\right)$ para $\mathbf{n}=(-1,0)$ ) como pode ser observado na figura A.1. Para o cálculo das velocidades pertencentes ao domínio $(\triangle \mathrm{e} \nabla)$ nos casos ilustrados na figura A.3, as velocidades normais e tangenciais encontram-se localizadas nas faces da célula [S] em questão. Logo, o uso das componentes da velocidade normal e tangencial na equação de diferenças finitas em (2.25) é natural.

Portanto, a resposta para o fato das velocidades tangenciais à superfície livre estarem localizadas em faces que separam duas células [E] (figuras A.1 e A.2) é justificada pela necessidade de utilizar estas velocidades nas equações discretizadas quando aplica-se a metodologia MAC sobre uma malha deslocada.

Outro aspecto importante na discretização espacial da metodologia GENSMAC próximo à superfície livre está relacionada ao gradiente da pressão na equação (2.22). De acordo com o 
ciclo computacional do GENSMAC, apresentado na subseção 2.7.1, o primeiro passo do método é calcular a pressão nas células de superfície livre. Esta pressão é calculada de acordo com a condição da tensão normal dada pela equação (2.7), que para fluidos newtonianos, é dada por (2.11) no caso bidimensional e em (2.14) no caso tridimensional. Neste caso, a pressão é calculada explicitamente, utilizando as velocidades já obtidas. Além disso, as equações (2.11) ou (2.14) são aproximadas no centro da célula da malha. Por exemplo, para o caso bidimensional, a equação (2.11) é discretizada no ponto $(i, j)$ onde está localizada a quantidade escalar (ver figuras A.1, A.2 e A.3)

Após obter o valor para pressão em todas as células [S], é necessário iniciar o segundo passo do ciclo computacional: resolver a equação de quantidade de movimento (2.25). E é neste momento, que outro aspecto importante deve ser lembrado: na metodologia GENSMAC, o gradiente da pressão é eliminado quando a equação (2.25) é aplicada em células [F].

Como ilustração deste fato, novamente por simplificação, considere o caso bidimensional. Inicialmente, aplique a equação (A.4) no ponto $\left(i+\frac{1}{2}, j\right)$ em uma célula [S] que tem uma célula vizinha à direita também $[\mathbf{S}]$, como representado na figura A.2. Note que as pressões utilizadas no cálculo de $\nabla p$ serão $p_{i+1, j}$ e $p_{i, j}$, ambas calculadas por (2.11). Entretanto, quando a equação de quantidade de movimento (A.4) for aplicada no ponto $\left(i+\frac{1}{2}, j\right)$ em uma célula do domínio, do tipo $[\mathbf{F}]$, a pressão tentativa, neste caso, não será calculada por (2.11), pois esta equação é aplicada apenas nas células da superfície livre. Logo, quando foi necessário o valor de $p$ em uma célula que não está na superfície livre, isto é, que não seja [S], a formulação GENSMAC define $p^{(n)}=0$. Portanto, em pontos onde existem duas células do tipo $[\mathbf{F}]$ vizinhas, o gradiente da pressão tentativa $\nabla p$ é eliminado da equação (2.25).

No caso onde tem-se uma célula $[\mathbf{F}]$ vizinha de uma célula $[\mathbf{S}]$, apenas o valor de $p$ em $[\mathbf{S}]$ é utilizado no cálculo de $\nabla p^{(n)}$. Métodos de projeção que eliminam o gradiente da pressão da equação (2.22) são baseados nos trabalhos de Chorin [23] e Temam [152] e conhecidos como métodos de projeção sem acréscimo da pressão, e foram também investigados, por exemplo por Kim e Moin [80] e Perot [125].

A eliminação do $\nabla p$ da equação de quantidade de movimento pode prejudicar a precisão dos resultados do método de projeção, quando aumenta-se o tamanho do passo temporal e não se utiliza as corretas condições de contorno para $\widetilde{\mathbf{u}}$ na equação (2.22). Esse fato foi discutido em $[134,13,61]$ que apresentaram alternativas para melhorar a precisão destes esquemas. Como a formulação GENSMAC é baseada em um esquema explícito não se pode aumentar o tamanho do passo temporal, e desta forma, nos trabalhos de Tomé e McKee [163] e de Tomé et al. [155] não foi possível notar a perda de precisão nos resultados, e nenhum tratamento especial das 
condições de contorno para $\widetilde{\mathbf{u}}$ na equação (2.25) foi realizado. Entretanto, quando aplica-se métodos implícitos, esse fato torna-se evidente, e será discutido a seguir.

Considere o problema da seção 3.4, onde um fluido newtoniano escoa sobre duas placas paralelas (Hagen-Poiseuille flow). Os dados para a simulação deste problema são os mesmos apresentados na seção 3.4. Entretanto, agora aplicou-se o método GENSMAC modificado introduzindo os métodos de EI e CN como apresentado na seção 2.8. As tabelas A.1 e A.2 mostram os resultados destas simulações. Nota-se que, por utilizar esquemas implícitos em um problema confinado (somente células do tipo $[\mathbf{F}]$ ), o método GENSMAC modificado permitiu a simulação deste problema com um passo temporal maior que o método explícito. Desta forma, pode-se verificar o aspecto da eliminação do gradiente de pressão da equação de quantidade de movimento, pois os erros aumentaram conforme aumentou-se $\delta t$. Portanto, é fundamental manter o gradiente de pressão na equação de quantidade de movimento quando aplica-se métodos implícitos. Todas as formulações implícitas desenvolvidas nesta tese utiliza na equação de quantidade de movimento o gradiente de pressão, permitindo melhores resultados como foi mencionado anteriormente nas seções de resultados numéricos.

Tabela A.1: Escoamento confinado $(R e=0.1)$.

\begin{tabular}{|c|c|c|c|}
\hline Método & $E r(u(y))$ & $\delta t$ & $\begin{array}{c}\text { Tempo de processamento } \\
\text { (minutos }: \text { segundos) }\end{array}$ \\
\hline GENSMAC & $1.5099 \times 10^{-3}$ & $2.5 \times 10^{-5}$ & $104: 31$ \\
GENSMAC-EI & $2.3011 \times 10^{-1}$ & $1.25 \times 10^{-2}$ & $3: 21$ \\
GENSMAC-CN & $3.1622 \times 10^{-2}$ & $1.25 \times 10^{-2}$ & $3: 00$ \\
\hline
\end{tabular}

Tabela A.2: Escoamento confinado $(R e=0.01)$.

\begin{tabular}{|c|c|c|c|}
\hline Método & $\operatorname{Er}(u(y))$ & $\delta t$ & $\begin{array}{c}\text { Tempo de processamento } \\
\text { (minutos }: \text { segundos) }\end{array}$ \\
\hline GENSMAC & $1.4817 \times 10^{-3}$ & $2.5 \times 10^{-6}$ & $874: 01$ \\
GENSMAC-EI & $3.0529 \times 10^{-1}$ & $1.25 \times 10^{-2}$ & $3: 00$ \\
GENSMAC-CN & $2.9814 \times 10^{-1}$ & $1.25 \times 10^{-2}$ & $3: 09$ \\
\hline
\end{tabular}

Os dois aspectos mencionados acima, aplicam-se da mesma forma para o caso tridimensional, e portanto, não serão apresentados neste trabalho. 


\section{A.2 Método semi-implícito estável utilizando EI}

Uma diferença importante da primeira formulação apresentada na seção 4.2 comparada ao método GENSMAC é a conservação do gradiente de pressão $\nabla p$ na equação (2.37). Na formulação SI, a pressão na superfície livre é tratada implicitamente (ver equação (4.3)), e portanto, tanto para as células [S], como para as células $[\mathbf{F}]$, mantém-se o gradiente de pressão na equação (2.37). A conservação do $\nabla p$ garantiu resultados mais precisos quando o passo do tempo foi aumentado.

A construção das equações de diferenças finitas para a formulação SI descrita em 4.2 segue as mesmas estratégias propostas por Tomé e McKee [163]. Apenas como ilustração, a equação de quantidade de movimento (2.37) na direção $x$ pode ser escrita como

$$
\begin{aligned}
\frac{1}{\delta t} \widetilde{u}_{i+\frac{1}{2}, j} & -\frac{1}{R e}\left(\frac{\widetilde{u}_{i-\frac{1}{2}, j}-2 \widetilde{u}_{i+\frac{1}{2}, j}+\widetilde{u}_{i+\frac{3}{2}, j}}{(\delta x)^{2}}+\frac{\widetilde{u}_{i+\frac{1}{2}, j-1}-2 \widetilde{u}_{i+\frac{1}{2}, j}+\widetilde{u}_{i+\frac{1}{2}, j+1}}{(\delta y)^{2}}\right) \\
& =\frac{1}{\delta t} u_{i+\frac{1}{2}, j}^{(n)}-(\mathcal{C}(u u)+\mathcal{C}(v u))^{(n)}-\left(\frac{p_{i+1, j}-p_{i, j}}{\delta x}\right)^{(n)}+\frac{1}{F r^{2}} g_{x},
\end{aligned}
$$

onde os termos convectivos $\mathcal{C}(u u)$ e $\mathcal{C}(v u)$ são aproximados pelo método upwind de alta ordem VONOS detalhado no trabalho de Ferreira et al. [47].

A equação de Poisson (2.28) é discretizada da seguinte forma

$$
\begin{aligned}
& \frac{\psi_{i+1, j}^{(n+1)}-2 \psi_{i, j}^{(n+1)}+\psi_{i-1, j}^{(n+1)}}{(\delta x)^{2}}+\frac{\psi_{i, j+1}^{(n+1)}-2 \psi_{i, j}^{(n+1)}+\psi_{i, j-1}^{(n+1)}}{(\delta y)^{2}} \\
& =\frac{1}{\delta t}\left(\frac{\widetilde{u}_{i+\frac{1}{2}, j}-\widetilde{u}_{i-\frac{1}{2}, j}}{\delta x}+\frac{\widetilde{v}_{i, j+\frac{1}{2}}-\widetilde{v}_{i, j-\frac{1}{2}}}{\delta y}\right) .
\end{aligned}
$$

Para $\psi^{(n+1)}$ na superfície livre, a equação (4.9) é discretizada como

$$
\begin{aligned}
& \left(1+\frac{4 \delta t}{(\delta y)^{2} R e}\right) \psi_{i, j}^{(n+1)}-\left(\frac{2 \delta t}{(\delta y)^{2} R e}\right) \psi_{i, j+1}^{(n+1)}-\left(\frac{2 \delta t}{(\delta y)^{2} R e}\right) \psi_{i, j-1}^{(n+1)}= \\
& -\frac{2}{R e}\left(\frac{\widetilde{v}_{i, j+\frac{1}{2}}-\widetilde{v}_{i, j-\frac{1}{2}}}{\delta y}\right)-p_{i, j}^{(n)} .
\end{aligned}
$$

No segundo caso, note que para a equação (4.13), tem-se

$$
\left.\left(\frac{\partial^{2} \psi^{(n+1)}}{\partial x \partial y}\right)\right|_{i, j}=\frac{\psi_{i, j}^{(n+1)}-\psi_{i, j-1}^{(n+1)}-\psi_{i-1, j}^{(n+1)}+\psi_{i-1, j-1}^{(n+1)}}{\delta x \delta y}
$$


e devido à malha deslocada,

$$
\begin{aligned}
& \left.\left(\frac{\partial \widetilde{u}}{\partial y}\right)\right|_{i, j}=\frac{\widetilde{u}_{i+\frac{1}{2}, j}+\widetilde{u}_{i-\frac{1}{2}, j}-\widetilde{u}_{i+\frac{1}{2}, j-1}-\widetilde{u}_{i-\frac{1}{2}, j-1}}{2 \delta y} \\
& \left.\left(\frac{\partial \widetilde{v}}{\partial x}\right)\right|_{i, j}=\frac{\widetilde{v}_{i, j+\frac{1}{2}}+\widetilde{v}_{i, j-\frac{1}{2}}-\widetilde{v}_{i-1, j+\frac{1}{2}}-\widetilde{v}_{i-1, j-\frac{1}{2}}}{2 \delta x} .
\end{aligned}
$$

Logo, substituindo estas aproximações em (4.13), tem-se

$$
\begin{aligned}
& \left(1+\frac{2 \delta t}{(\delta x \delta y) R e}\right) \psi_{i, j}^{(n+1)}-\left(\frac{2 \delta t}{(\delta x \delta y) R e}\right) \psi_{i, j-1}^{(n+1)}-\left(\frac{2 \delta t}{(\delta x \delta y) R e}\right) \psi_{i-1, j}^{(n+1)}+ \\
& \left(\frac{2 \delta t}{(\delta x \delta y) R e}\right) \psi_{i-1, j-1}^{(n+1)}=\left(\frac{1}{R e}\right)\left(\frac{\widetilde{u}_{i+\frac{1}{2}, j}+\widetilde{u}_{i-\frac{1}{2}, j}-\widetilde{u}_{i+\frac{1}{2}, j-1}-\widetilde{u}_{i-\frac{1}{2}, j-1}}{2 \delta y}\right. \\
& \left.+\frac{\widetilde{v}_{i, j+\frac{1}{2}}+\widetilde{v}_{i, j-\frac{1}{2}}-\widetilde{v}_{i-1, j+\frac{1}{2}}-\widetilde{v}_{i-1, j-\frac{1}{2}}}{2 \delta x}\right)-p_{i, j}^{(n)} .
\end{aligned}
$$

O sistema linear resultante da discretização da equação de Poisson (A.6) adicionadas das equações (A.7) e (A.10) é esparso e não-simétrico, e neste trabalho é resolvido pelo método dos gradientes bi-conjugados com o pré-condicionador de Jacobi [130].

Apenas como ilustração, a equação (2.27) é discretizada na direção $x$ como

$$
u_{i+\frac{1}{2}, j}^{(n+1)}=\widetilde{u}_{i+\frac{1}{2}, j}-\delta t \frac{\psi_{i+1, j}^{(n+1)}-\psi_{i, j}^{(n+1)}}{\delta x},
$$

e a equação da atualização de pressão (2.32)

$$
p_{i, j}^{(n+1)}=p_{i, j}^{(n)}+\psi_{i, j}^{(n+1)} .
$$

Detalhes sobre a discretizações das demais equações envolvidas no método semi-implícito podem ser encontrados em [113].

\section{A.3 Método semi-implícito estável utilizando CN}

A construção das equações de diferenças finitas para esta formulação é muito semelhante à primeira formulação apresentada anteriormente. Logo, nesta segunda formulação, também conserva-se o gradiente de pressão $\nabla p^{(n)}$ na equação (2.38). Desta forma, a equação de quan- 
tidade de movimento (2.38) na direção $x$ é

$$
\begin{aligned}
& \frac{1}{\delta t} \widetilde{u}_{i+\frac{1}{2}, j}-\frac{1}{2 R e}\left(\frac{\widetilde{u}_{i-\frac{1}{2}, j}-2 \widetilde{u}_{i+\frac{1}{2}, j}+\widetilde{u}_{i+\frac{3}{2}, j}}{(\delta x)^{2}}+\frac{\widetilde{u}_{i+\frac{1}{2}, j-1}-2 \widetilde{u}_{i+\frac{1}{2}, j}+\widetilde{u}_{i+\frac{1}{2}, j+1}}{(\delta y)^{2}}\right) \\
& =\frac{1}{\delta t} u_{i+\frac{1}{2}, j}^{(n)}+\frac{1}{2 R e}\left(\frac{u_{i-\frac{1}{2}, j}-2 u_{i+\frac{1}{2}, j}+u_{i+\frac{3}{2}, j}}{(\delta x)^{2}}+\frac{u_{i+\frac{1}{2}, j-1}-2 u_{i+\frac{1}{2}, j}+u_{i+\frac{1}{2}, j+1}}{(\delta y)^{2}}\right)^{(n)} \\
& -(\mathcal{C}(u u)+\mathcal{C}(v u))^{(n)}-\left(\frac{p_{i+1, j}-p_{i, j}}{\delta x}\right)^{(n)}+\frac{1}{F r^{2}} g_{x},
\end{aligned}
$$

onde os termos convectivos $\mathcal{C}(u u)$ e $\mathcal{C}(v u)$ são aproximados pelo método upwind de alta ordem QUICKEST adaptativo detalhado nos trabalhos de Ferreira et al. [48, 46].

A equação de Poisson discretizada foi apresentada em (A.6). A equação (4.18) é discretizada como

$$
\begin{aligned}
& \left(1+\frac{\delta t}{R e(\delta x)^{2}}+\frac{\delta t}{R e(\delta y)^{2}}+\frac{4 \delta t}{(\delta y)^{2} R e}\right) \psi_{i, j}^{(n+1)}-\left(\frac{\delta t}{2(\delta y)^{2} R e}\right) \psi_{i-1, j}^{(n+1)} \\
& -\left(\frac{\delta t}{2(\delta y)^{2} R e}+\frac{2 \delta t}{(\delta y)^{2} R e}\right) \psi_{i, j+1}^{(n+1)}-\left(\frac{\delta t}{2(\delta y)^{2} R e}+\frac{2 \delta t}{(\delta y)^{2} R e}\right) \psi_{i, j-1}^{(n+1)} \\
& =-\frac{2}{R e}\left(\frac{\widetilde{v}_{i, j+\frac{1}{2}}-\widetilde{v}_{i, j-\frac{1}{2}}}{\delta y}\right)-p_{i, j}^{(n)} .
\end{aligned}
$$

Na equação (4.18) discretiza-se o operador laplaciano utilizando 5 pontos, no entanto em (A.14) a pressão tentativa $\psi_{i+1, j}^{(n+1)}$ não aparece pois assume-se que este valor é igual a zero nas células [E]. No segundo caso, a equação (4.19) é discretizada da seguinte forma

$$
\begin{aligned}
& \left(1+\frac{\delta t}{(\delta x)^{2} R e}+\frac{\delta t}{(\delta x)^{2} R e}+\frac{2 \delta t}{(\delta x \delta y) R e}\right) \psi_{i, j}^{(n+1)}+\left(\frac{2 \delta t}{(\delta x \delta y) R e}\right) \psi_{i-1, j-1}^{(n+1)} \\
& -\left(\frac{\delta t}{2(\delta y)^{2} R e}+\frac{2 \delta t}{(\delta x \delta y) R e}\right) \psi_{i, j-1}^{(n+1)}-\left(\frac{\delta t}{2(\delta x)^{2} R e}+\frac{2 \delta t}{(\delta x \delta y) R e}\right) \psi_{i-1, j}^{(n+1)} \\
& =\frac{1}{R e}\left(\left.\frac{\partial \widetilde{u}}{\partial y}\right|_{i, j}+\left.\frac{\partial \widetilde{v}}{\partial x}\right|_{i, j}\right)-p_{i, j}^{(n)} .
\end{aligned}
$$

Novamente, o termo de derivada mista para $\psi$ na equação (4.19) é discretizado como em (A.8). Entretanto, nesta nova formulação os termos das derivadas das velocidades intermediárias são obtidos pelas aproximações

$$
\left.\frac{\partial \widetilde{u}}{\partial y}\right|_{i, j}=\frac{\widetilde{u}_{i, j}-\widetilde{u}_{i, j-1}}{\delta y},\left.\quad \frac{\partial \widetilde{v}}{\partial x}\right|_{i, j}=\frac{\widetilde{v}_{i, j}-\widetilde{v}_{i-1, j}}{\delta x} .
$$


Nas equações (A.16), os valores das velocidades intermediárias no centro das células são aproximados por

$$
\widetilde{u}_{i, j} \approx \widetilde{u}_{i-\frac{1}{2}, j}, \quad \widetilde{u}_{i, j-1} \approx \widetilde{u}_{i-\frac{1}{2}, j-1}, \quad \widetilde{v}_{i, j} \approx \widetilde{v}_{i, j-\frac{1}{2}}, \widetilde{v}_{i-1, j} \approx \widetilde{v}_{i-1, j-\frac{1}{2}}
$$

Portanto, nesta formulação, a equação (A.15) com as aproximações acima, é utilizada para o segundo caso ilustrado na figura A.3a).

Para o campo de velocidade final $\mathbf{u}^{(n+1)}$ as equações de diferenças finitas são as mesmas utilizadas na formulação anterior (ver equação (A.11)), enquanto que a fórmula para a atualização da pressão (4.17) é discretizada como

$$
p_{i, j}^{(n+1)}=p_{i, j}^{(n)}+\psi_{i, j}^{(n+1)}-\frac{\delta t}{2 R e}\left(\frac{\psi_{i+1, j}^{(n+1)}-2 \psi_{i, j}^{(n+1)}+\psi_{i-1, j}^{(n+1)}}{(\delta x)^{2}}+\frac{\psi_{i, j+1}^{(n+1)}-2 \psi_{i, j}^{(n+1)}+\psi_{i, j-1}^{(n+1)}}{(\delta y)^{2}}\right) .
$$

\section{A.4 Extensão para problemas tridimensionais newtonianos e viscoelásticos}

A construção das aproximações de diferenças finitas das equações envolvidas no caso tridimensional segue o mesmo desenvolvimento do caso bidimensional. Exemplo de uma célula computacional para o caso tridimensional em uma malha deslocada foi apresentado na figura $2.2 \mathrm{~b}$ ). Note que as velocidades são aproximadas nas faces do cubo, enquanto que o tensor tensão não-newtoniano $\mathbf{S}$ e a pressão são aproximados no centro da célula onde está localizado $\chi_{i, j, k}$ na figura $\left.2.2 \mathrm{~b}\right)$.

A equação de quantidade de movimento (5.11) é aproximada da seguinte forma: os termos viscosos são aproximados pelo esquema de Euler implícito ou Crank-Nicolson, dependendo dos valores de $\theta_{1}$ e $\theta_{4}$, enquanto que o gradiente de pressão e a divergência do tensor tensão não-newtoniano $\mathbf{S}$ são aproximados utilizando diferenças centrais. Os termos convectivos são aproximados pelo esquema upwind de alta ordem CUBISTA de Alves et al. [3]. Detalhes das equações de diferenças finitas para o método CUBISTA podem ser encontrados em [44]. Neste 
caso, a equação de quantidade de movimento na direção $x$ é aproximada por

$$
\begin{aligned}
& \frac{\widetilde{u}_{i+\frac{1}{2}, j, k}}{\delta t}-\theta_{1} \frac{\gamma}{R e}\left(\frac{\widetilde{u}_{i+\frac{3}{2}, j, k}-2 \widetilde{u}_{i+\frac{1}{2}, j, k}+\widetilde{u}_{i-\frac{1}{2}, j, k}}{(\delta x)^{2}}+\frac{\widetilde{u}_{i+\frac{1}{2}, j+1, k}-2 \widetilde{u}_{i+\frac{1}{2}, j, k}+\widetilde{u}_{i+\frac{1}{2}, j-1, k}}{(\delta y)^{2}}\right. \\
& \left.+\frac{\widetilde{u}_{i+\frac{1}{2}, j, k+1}-2 \widetilde{u}_{i+\frac{1}{2}, j, k}+\widetilde{u}_{i+\frac{1}{2}, j, k-1}}{(\delta z)^{2}}\right)=\frac{u_{i+\frac{1}{2}, j, k}^{(n)}}{\delta t}-(\mathcal{C}(u u)+\mathcal{C}(v u)+\mathcal{C}(w u))^{(n)} \\
& -\left(\frac{p_{i+1, j, k}-p_{i, j, k}}{\delta x}\right)^{(n)}+\theta_{4} \frac{\gamma}{R e}\left(\frac{u_{i+\frac{3}{2}, j, k}-2 u_{i+\frac{1}{2}, j, k}+u_{i-\frac{1}{2}, j, k}}{(\delta x)^{2}}\right. \\
& \left.+\frac{u_{i+\frac{1}{2}, j+1, k}-2 u_{i+\frac{1}{2}, j, k}+u_{i+\frac{1}{2}, j-1, k}}{(\delta y)^{2}}+\frac{u_{i+\frac{1}{2}, j, k+1}-2 u_{i+\frac{1}{2}, j, k}+u_{i+\frac{1}{2}, j, k-1}}{(\delta z)^{2}}\right)^{(n)} \\
& +\beta\left(\frac{S_{i+1, j, k}^{x x}-S_{i, j, k}^{x x}}{\delta x}+\frac{S_{i+\frac{1}{2}, j+\frac{1}{2}, k}^{y x}-S_{i+\frac{1}{2}, j-\frac{1}{2}, k}^{y x}}{\delta y}+\frac{S_{i+\frac{1}{2}, j, k+\frac{1}{2}}^{z x}-S_{i+\frac{1}{2}, j, k-\frac{1}{2}}^{z x}}{\delta z}+\frac{1}{F r^{2}} g_{x} .\right.
\end{aligned}
$$

Termos como $S_{i+\frac{1}{2}, j+\frac{1}{2}, k}^{y x}$ são obtidos pela média dos seus vizinhos mais próximos, por exemplo

$$
S_{i+\frac{1}{2}, j+\frac{1}{2}, k}^{y x}=\frac{S_{i, j, k}^{y x}+S_{i+1, j, k}^{y x}+S_{i, j+1, k}^{y x}+S_{i+1, j+1, k}^{y x}}{4}
$$

As equações de quantidade de movimento nas direções $y$ e $z$ são obtidas da mesma forma. A equação de Poisson (2.28) é discretizada pela equação de diferenças de segunda ordem

$$
\begin{aligned}
& \frac{\psi_{i+1, j, k}^{(n+1)}-2 \psi_{i, j, k}^{(n+1)}+\psi_{i-1, j, k}^{(n+1)}}{(\delta x)^{2}}+\frac{\psi_{i, j+1, k}^{(n+1)}-2 \psi_{i, j, k}^{(n+1)}+\psi_{i, j-1, k}^{(n+1)}}{(\delta y)^{2}} \\
& +\frac{\psi_{i, j, k+1}^{(n+1)}-2 \psi_{i, j, k}^{(n+1)}+\psi_{i, j, k-1}^{(n+1)}}{(\delta z)^{2}}=t^{-1}\left(\frac{\widetilde{u}_{i+\frac{1}{2}, j, k}-\widetilde{u}_{i-\frac{1}{2}, j, k}}{\delta x}\right. \\
& \left.+\frac{\widetilde{v}_{i, j+\frac{1}{2}, k}-\widetilde{v}_{i, j-\frac{1}{2}, k}}{\delta y}+\frac{\widetilde{w}_{i, j, k+\frac{1}{2}}-\widetilde{w}_{i, j, k-\frac{1}{2}}}{\delta z}\right)
\end{aligned}
$$

A equação (A.20) é aplicada em todas células $[\mathbf{F}]$ do domínio. O valor de $\psi_{i, j, k}^{(n+1)}$ nas células [S] é calculado pelas equações descritas anteriormente (5.19), (5.23) e (5.26) com aproximações apropriadas para cada tipo de superfície, isto é, superfície plana $1 \mathrm{D}, 2 \mathrm{D}$ ou $3 \mathrm{D}$, respectivamente. Por exemplo, considere uma célula $[\mathbf{S}]$ contendo apenas a face superior em contato com uma célula $[\mathrm{E}]$ conforme descrito na figura A.4, assumindo que neste caso a superfície livre é aproximada por uma superfície planar $\mathbf{1 D}$ paralela aos eixos $x$ e $y$ com $\mathbf{n}=(0,0,1)$. Neste 


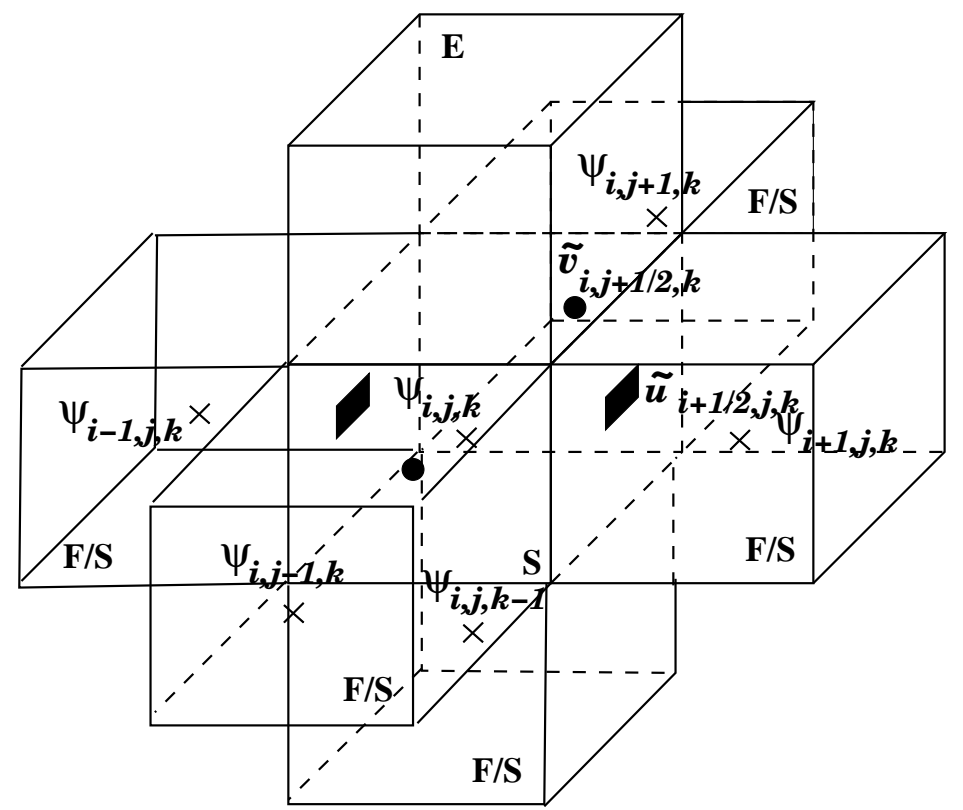

Figura A.4: Célula [S] com apenas a face superior em contato com uma célula [E].

caso, $\psi_{i, j, k}^{(n+1)}$ é dado pela equação (5.19) que é aproximada da seguinte forma

$$
\begin{aligned}
& {\left[1+\left(\frac{\gamma \delta t}{\operatorname{Re}}\right)\left(\frac{5}{(\delta x)^{2}}+\frac{5}{(\delta y)^{2}}+\frac{1}{(\delta z)^{2}}\right)\right] \psi_{i, j, k}^{(n+1)}-\left(\frac{5 \gamma \delta t}{2 \operatorname{Re}(\delta x)^{2}}\right)\left[\psi_{i+1, j, k}^{(n+1)}+\psi_{i-1, j, k}^{(n+1)}\right]} \\
& -\left(\frac{5 \gamma \delta t}{2 \operatorname{Re}(\delta y)^{2}}\right)\left[\psi_{i, j+1, k}^{(n+1)}+\psi_{i, j-1, k}^{(n+1)}\right]-\left(\frac{\gamma \delta t}{2 \operatorname{Re}(\delta z)^{2}}\right) \psi_{i, j, k-1}^{(n+1)} \\
& =\frac{-2 \gamma}{\operatorname{Re}}\left(\frac{\widetilde{u}_{i+\frac{1}{2}, j, k}-\widetilde{u}_{i-\frac{1}{2}, j, k}}{\delta x}+\frac{\widetilde{v}_{i, j+\frac{1}{2}, k}-\widetilde{v}_{i, j-\frac{1}{2}, k}}{\delta y}\right)-p_{i, j, k}^{(n)}+\beta\left(S^{z z}\right)_{i, j, k}^{(n)} .
\end{aligned}
$$

$\mathrm{Na}$ equação (A.21) a pressão tentativa $\psi_{i, j, k+1}^{(n+1)}$ não aparece pois é assumido que nas células [E] a pressão tentativa é definida como zero. As equações de diferenças finitas para as outras configurações da superfície planar 1D são obtidas de forma semelhante.

Para as células $[\mathbf{S}]$ contendo duas faces adjacentes em contato com células [E] a pressão tentativa $\psi_{i, j, k}^{(n+1)}$ é calculada utilizando a equação derivada para o caso da superfície planar 2D. Neste caso, se uma célula [S] tem as faces $\left(i+\frac{1}{2}\right)$ e $\left(k+\frac{1}{2}\right)$ em contato com faces de célula [E] então $\psi_{i, j, k}^{(n+1)}$ é calculada da equação (5.23) aplicada no centro da célula [S]. As derivadas segunda são aproximadas por diferenças de segunda ordem enquanto que as derivadas $\frac{\partial^{2} \psi^{(n+1)}}{\partial x \partial z}$, 
$\frac{\partial \widetilde{u}}{\partial z}$ e $\frac{\partial \widetilde{w}}{\partial x}$ são substituídas, respectivamente, por aproximações de primeira ordem

$$
\begin{gathered}
\left.\frac{\partial^{2} \psi^{(n+1)}}{\partial x \partial z}\right|_{i, j, k}=\frac{\psi_{i, j, k}^{(n+1)}-\psi_{i-1, j, k}^{(n+1)}-\psi_{i, j, k-1}^{(n+1)}+\psi_{i-1, j, k-1}^{(n+1)}}{\delta x \delta z} \\
\left.\frac{\partial \widetilde{u}}{\partial z}\right|_{i, j, k}=\frac{\widetilde{u}_{i, j, k}-\widetilde{u}_{i, j, k-1}}{\delta z},\left.\quad \frac{\partial \widetilde{w}}{\partial x}\right|_{i, j, k}=\frac{\widetilde{w}_{i, j, k}-\widetilde{w}_{i-1, j, k}}{\delta x} .
\end{gathered}
$$

Em (A.23), os valores das velocidades intermediárias $\widetilde{\mathbf{u}}$ no centro das células são aproximados por

$$
\widetilde{u}_{i, j, k} \approx \widetilde{u}_{i-\frac{1}{2}, j, k}, \widetilde{u}_{i, j, k-1} \approx \widetilde{u}_{i-\frac{1}{2}, j, k-1}, \quad \widetilde{w}_{i, j, k} \approx \widetilde{w}_{i, j, k-\frac{1}{2}}, \widetilde{w}_{i-1, j, k} \approx \widetilde{w}_{i-1, j, k-\frac{1}{2}} .
$$

Logo, introduzindo essas aproximações na equação (5.23) e reagrupando termos semelhantes, obtém-se

$$
\begin{aligned}
& {\left[1+\left(\frac{\gamma \delta t}{R e}\right)\left(\frac{1}{(\delta x)^{2}}+\frac{3}{(\delta y)^{2}}+\frac{1}{(\delta z)^{2}}+\frac{2}{\delta x \delta z}\right)\right] \psi_{i, j, k}^{(n+1)}} \\
& -\left(\frac{\gamma \delta t}{R e}\right)\left(\frac{1}{2(\delta x)^{2}}+\frac{2}{\delta x \delta z}\right) \psi_{i-1, j, k}^{(n+1)}-\left(\frac{3 \gamma \delta t}{2 R e(\delta y)^{2}}\right)\left[\psi_{i, j+1, k}^{(n+1)}+\psi_{i, j-1, k}^{(n+1)}\right] \\
& -\left(\frac{\gamma \delta t}{R e}\right)\left(\frac{1}{2(\delta z)^{2}}+\frac{2}{\delta x \delta z}\right) \psi_{i, j, k-1}^{(n+1)}+\left(\frac{2 \gamma \delta t}{\operatorname{Re} \delta x \delta z}\right) \psi_{i-1, j, k-1}^{(n+1)}= \\
& \frac{-\gamma}{\operatorname{Re}}\left(\frac{\widetilde{v}_{i, j+\frac{1}{2}, k}-\widetilde{v}_{i, j-\frac{1}{2}, k}}{\delta y}-\left.\frac{\partial \widetilde{u}}{\partial z}\right|_{i, j, k}-\left.\frac{\partial \widetilde{w}}{\partial x}\right|_{i, j, k}\right) \\
& -p_{i, j, k}^{(n)}+\beta\left[\frac{1}{2} S_{i, j, k}^{x x}+\frac{1}{2} S_{i, j, k}^{z z}+S_{i, j, k}^{x z}\right]^{(n)} .
\end{aligned}
$$

Novamente, nota-se que os valores de $\psi_{i+1, j, k}^{(n+1)}$ e $\psi_{i, j, k+1}^{(n+1)}$ não aparecem na equação de diferença pois neste caso assume-se que em células vazias $\psi=0$. As equações para $\psi_{i, j, k}^{(n+1)}$ nas células [S] com duas células [E] vizinhas, mas em configurações diferentes, são construídas da mesma forma.

No caso de células $[\mathbf{S}]$ contendo três faces em contato com células $[\mathbf{E}]$, a equação de diferenças finitas para $\psi^{(n+1)}$ é obtida da mesma forma que anteriormente. Para isto, considere uma célula $[\mathbf{S}]$ com as faces $\left(i+\frac{1}{2}\right),\left(j+\frac{1}{2}\right)$ e $\left(k+\frac{1}{2}\right)$ em contato com células [E]; logo a pressão tentativa $\psi_{i, j, k}^{(n+1)}$ é calculada da equação (5.26). Para aproximar esta equação por diferenças finitas procede-se como no caso de células $[\mathbf{S}]$ contendo duas faces adjacentes em contato com células $[\mathbf{E}]$. As derivadas cruzadas são aproximadas por diferenças de primeira ordem, como realizado em (A.22), e as derivadas de segunda ordem são aproximadas por diferenças centrais. 
Desta forma, a equação (5.26) é aproximada pela seguinte equação de diferenças:

$$
\begin{aligned}
& {\left[1+\left(\frac{\gamma \delta t}{R e}\right)\left(\frac{1}{(\delta x)^{2}}+\frac{1}{(\delta y)^{2}}+\frac{1}{(\delta z)^{2}}+\frac{4}{3 \delta x \delta y}+\frac{4}{3 \delta x \delta z}+\frac{4}{3 \delta y \delta z}\right)\right] \psi_{i, j, k}^{(n+1)}} \\
& -\left(\frac{\gamma \delta t}{R e}\right)\left(\frac{1}{2(\delta x)^{2}}+\frac{4}{3 \delta x \delta y}+\frac{4}{3 \delta x \delta z}\right) \psi_{i-1, j, k}^{(n+1)} \\
& -\left(\frac{\gamma \delta t}{R e}\right)\left(\frac{1}{2(\delta y)^{2}}+\frac{4}{3 \delta x \delta y}+\frac{4}{3 \delta y \delta z}\right) \psi_{i, j-1, k}^{(n+1)} \\
& -\left(\frac{\gamma \delta t}{R e}\right)\left(\frac{1}{2(\delta z)^{2}}+\frac{4}{3 \delta x \delta z}+\frac{4}{3 \delta y \delta z}\right) \psi_{i, j, k-1}^{(n+1)}+\left(\frac{\gamma \delta t}{R e}\right) \frac{4}{3 \delta x \delta y} \psi_{i-1, j-1, k}^{(n+1)} \\
& +\left(\frac{\gamma \delta t}{R e}\right) \frac{4}{3 \delta x \delta z} \psi_{i-1, j, k-1}^{(n+1)}+\left(\frac{\gamma \delta t}{R e}\right) \frac{4}{3 \delta y \delta z} \psi_{i, j-1, k-1}^{(n+1)} \\
& =\frac{2 \gamma}{3 R e}\left(\left.\frac{\partial \widetilde{u}}{\partial y}\right|_{i, j, k}+\left.\frac{\partial \widetilde{u}}{\partial z}\right|_{i, j, k}+\left.\frac{\partial \widetilde{v}}{\partial x}\right|_{i, j, k}+\left.\frac{\partial \widetilde{v}}{\partial z}\right|_{i, j, k}+\left.\frac{\partial \widetilde{w}}{\partial x}\right|_{i, j, k}+\left.\frac{\partial \widetilde{w}}{\partial y}\right|_{i, j, k}\right) \\
& -p_{i, j, k}^{(n)}+\beta \frac{1}{3}\left[S_{i, j, k}^{x x}+S_{i, j, k}^{y y}+S_{i, j, k}^{z z}+2\left(S_{i, j, k}^{x y}+S_{i, j, k}^{x z}+S_{i, j, k}^{y z}\right)\right]^{(n)} .
\end{aligned}
$$

Os outros casos de células $[\mathbf{S}]$ contendo três lados adjacentes com células $[\mathbf{E}]$ são derivados analogamente.

A equação de diferenças finitas para a atualização do campo de velocidade no caso tridimensional é semelhante à equação discreta do caso bidimensional (A.11), enquanto que a equação para a atualização da pressão (5.12) é discretizada da seguinte forma

$$
\begin{aligned}
p_{i, j, k}^{(n+1)} & =p_{i, j, k}^{(n)}+\psi_{i, j, k}^{(n+1)}-\gamma \frac{\delta t}{2 R e}\left(\frac{\psi_{i+1, j, k}^{(n+1)}-2 \psi_{i, j, k}^{(n+1)}+\psi_{i-1, j, k}^{(n+1)}}{(\delta x)^{2}}\right. \\
& \left.+\frac{\psi_{i, j+1, k}^{(n+1)}-2 \psi_{i, j, k}^{(n+1)}+\psi_{i, j-1, k}^{(n+1)}}{(\delta y)^{2}}+\frac{\psi_{i, j, k+1}^{(n+1)}-2 \psi_{i, j, k}^{(n+1)}+\psi_{i, j, k-1}^{(n+1)}}{(\delta z)^{2}}\right)
\end{aligned}
$$

As aproximações por diferenças finitas para as equações (5.3) e (5.4) do tensor tensão não-newtoniano $\mathbf{S}$ podem ser encontradas, respectivamente, nos trabalhos de Silva e Tomé [143] e Tomé et al. [165] para o modelo Maxwell, e em [156, 157] para o modelo Oldroyd-B. Como o objetivo deste trabalho não é derivar métodos para o tratamento destas equações, não será apresentado detalhes destas discretizações. Além disso, as equações de diferenças finitas utilizadas no último passo do algoritmo, apresentadas na subseção 5.2.1 podem ser encontradas em [155]. 


\section{A.5 Método de projeção de ordem dois para a formulação SI-AB $/ \mathrm{CN}$}

A construção das equações de diferenças finitas nesta formulação segue a mesma lógica da seção A.3, e portanto não será apresentada nesta tese. As principais modificações nesta formulação estão relacionadas com a discretização temporal, e não espacial.

\section{A.6 Método de projeção de ordem dois para a formulação laplaciano superficial}

A principal modificação nesta formulação está na construção da equação de diferenças para $\psi^{(n+1)}$ na superfície livre nos diferentes casos ilustrados nas figuras A.1 e A.2. As demais equações de diferenças finitas são construidas de maneira análoga ao que foi realizado anteriormente. Desta forma, nesta seção será apresentada apenas como utilizar as equações de diferenças finitas para a equação (6.28).

Considere a equação (6.28) discretizada de maneira generalizada como

$$
\begin{aligned}
& \left(1+\frac{2 \delta t}{(\delta s)^{2} R e}\right) \psi_{i, j}^{(n+1)}-\left(\frac{\delta t}{(\delta s)^{2} R e}\right) \psi_{1}^{(n+1)}-\left(\frac{\delta t}{(\delta s)^{2} R e}\right) \psi_{2}^{(n+1)}= \\
& -\frac{1}{R e}\left(\frac{h\left(\widetilde{u}_{m}\right)}{\delta s}+\frac{h\left(u_{m}^{(n)}\right)}{\delta s}\right)-p_{i, j}^{\left(n-\frac{1}{2}\right)} .
\end{aligned}
$$

A estrutura utilizada na equação (A.28) realça uma vantagem fundamental na formulação laplaciano superficial: a solução de um sistema linear simétrico tridiagonal para $\psi$. Nas demais formulações, as equações para $\psi$ na superfície livre resultam em sistemas lineares não-simétricos.

A tabela A.3 apresenta os valores de $\psi_{1}, \psi_{2}, h\left(\widetilde{u}_{m}\right)$ e $h\left(u_{m}^{(n)}\right)$. Em todas os casos, utiliza-se $\delta s \simeq \delta x$.

Tabela A.3: Valores de $\psi_{1}, \psi_{2}, h\left(\widetilde{u}_{m}\right)$ e $h\left(u_{m}^{(n)}\right)$ na equação de diferenças finitas (A.28) para os diferentes valores do vetor $\mathbf{n}$.

\begin{tabular}{|c|c|c|c|c|}
\hline $\mathbf{n}=\left(n_{x}, n_{y}\right)$ & $\psi_{1}$ & $\psi_{2}$ & $h\left(\widetilde{u}_{m}\right)$ & $h\left(u_{m}^{(n)}\right)$ \\
\hline$( \pm 1,0)$ & $\psi_{i, j+1}$ & $\psi_{i, j-1}$ & $\left(\widetilde{v}_{i, j+\frac{1}{2}}-\widetilde{v}_{i, j-\frac{1}{2}}\right)$ & $\left(v_{i, j+\frac{1}{2}}^{(n)}-v_{i, j-\frac{1}{2}}^{(n)}\right)$ \\
$(0, \pm 1)$ & $\psi_{i+1, j}$ & $\psi_{i-1, j}$ & $\left(\widetilde{u}_{i+\frac{1}{2}, j}-\widetilde{u}_{i-\frac{1}{2}, j}\right)$ & $\left(u_{i+\frac{1}{2}, j}^{(n)}-u_{i-\frac{1}{2}, j}^{(n)}\right)$ \\
\hline
\end{tabular}

